\title{
Dynamic Coordination Architecture and Mobility Management for Next Generation Cooperative Cellular Networks
}

\author{
by \\ Baha Uddin Kazi
}

A thesis submitted to the Faculty of Graduate and Postdoctoral Affairs in partial fulfillment of the requirements for the degree of

\author{
Doctor of Philosophy \\ in
}

Electrical and Computer Engineering

Carleton University

Ottawa, Ontario, Canada

(C) 2018, Baha Uddin Kazi 


\begin{abstract}
The fundamental challenges of existing cellular wireless networks are the exponential demand of mobile data traffic, higher data rates, massive numbers of user-coverage and lower latency. Moreover, the next generation of wireless cellular networks also consider potential use cases, such as autonomous vehicle control, smart cities, remote surgery and eHealth, tactile internet, etc. To address these challenges and potential use cases, network densification such as ultra-dense heterogeneous networks (UDHetNet) and multi-cell cooperation are considered as the foundation to support the $1000 \times$ capacity challenge in the next generation wireless cellular networks.
\end{abstract}

In this thesis, we study the coordination architecture and mobility management of multicell cooperative communications and present novel algorithms to improve the performance of multi-cell cooperative cellular networks. We propose DCEC: Direct CSI-feedback to Elected Coordination-station, a CoMP coordination architecture for cooperative communication to improve the performance of cellular networks, reducing the signaling overhead and latency. We extended the DCEC approach to heterogeneous cellular networks named DCEC-HetNet as well. We also propose a handover procedure for heterogeneous multi-cell cooperative cellular networks named EHoLM: Enhanced Handover for Low and Moderate speed UEs. The goal of the EHoLM handover procedure is to improve the system performance and user experience, reducing the number of handovers, handover oscillation and handover failure rate. To examine the performance of the proposed algorithms we use the discrete event system specifications (DEVS) for modeling and simulation of cellular networks employing the DCEC and EHoLM methods. 
Simulation results show that the proposed algorithms have potentials to improve the performance of cooperative cellular networks compared to the conventional methods.

We also study the verification and validation $(\mathrm{V} \& \mathrm{~V})$ process of simulation models. A revised lifecycle of modeling and simulation $(\mathrm{M} \& S)$ has also been presented that accommodates both formal and conceptual approaches of the verification and validation $(V \& V)$ process. Finally, how we validated the simulation models we developed for analyzing the proposed algorithms has been presented. 


\section{Acknowledgements}

First of all, I would like to thank Allah, the Almighty. I would particularly like to address my very special gratitude to my supervisor, professor Dr. Gabriel A. Wainer for his guidance and assistance throughout this research. You also gave me a large number of opportunities and challenges in the past five years that enables me to improve both academic and personal skills. Without your support, guidelines and knowledge sharing this thesis would have not been exist.

I also acknowledge the support of Ericsson Canada in the accomplishment of my research. My sincere thanks to Dr. Gary Boudreau and Ronald Casselman for their insightful comments and valuable feedbacks.

I would like to thank my friends and colleagues from ARS-Lab for all of their supports. The thesis wouldn't be what it is without the great support from all of you.

Last but surely not least, I am deeply grateful to my family and friends for their priceless support and encouragement, and to my wife and daughter, whose patience, understanding and support throughout this work was amazing. 


\section{Table of Contents}

Abstract......................................................................................................................................... iii

Acknowledgements ................................................................................................................. v

Table of Contents .......................................................................................................... vii

List of Tables ............................................................................................................................. xi

List of Figures.............................................................................................................................. xii

List of Equations ......................................................................................................................... xvi

List of Acronyms .......................................................................................................................... xviii

List of Symbols ........................................................................................................................ xxii

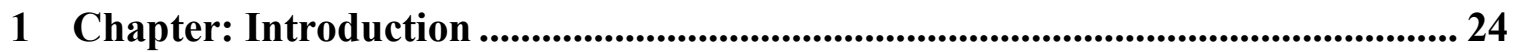

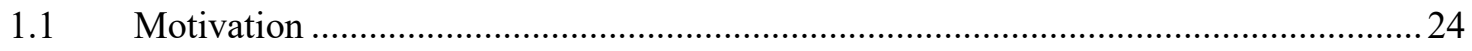

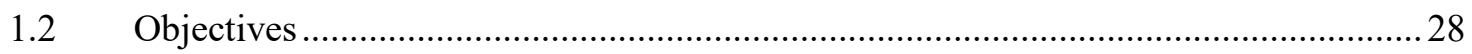

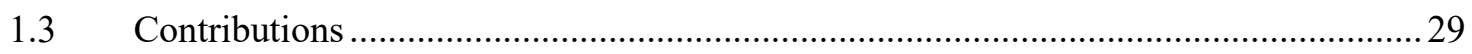

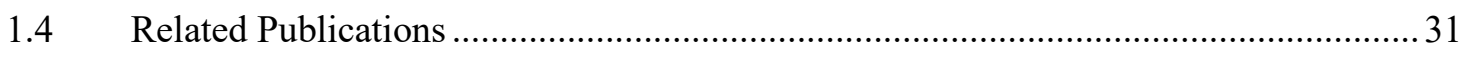

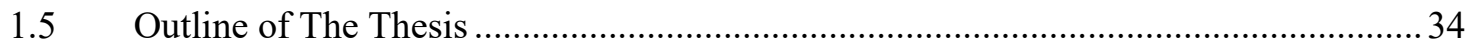

2 Chapter: Background and State of The Art............................................................... 35

2.1 Evolution of Cellular Technologies .......................................................................... 37

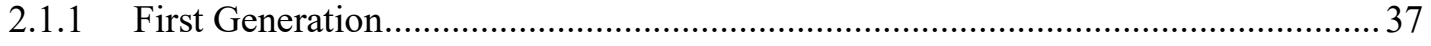

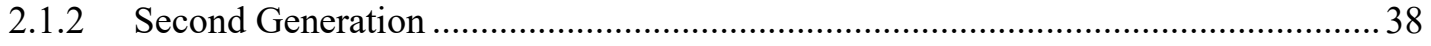

2.1.3 Third Generation ……………………………………………………………..... 39

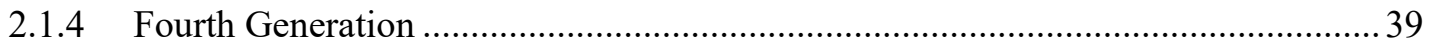

2.1.5 Next Generation Wireless Cellular Networks ……………………………………....42

2.1.5.1 5G Key Technology Enablers ………………………………………… 44

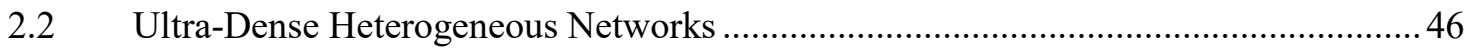




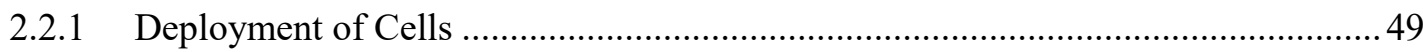

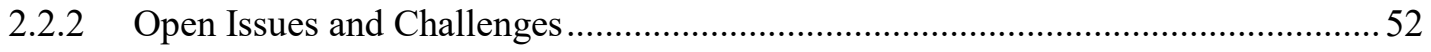

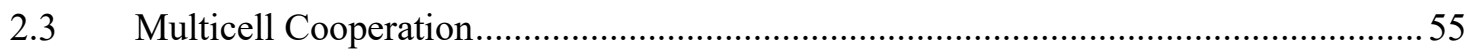

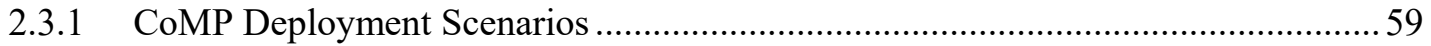

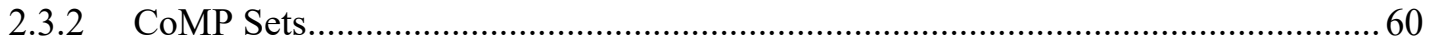

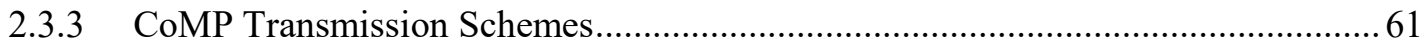

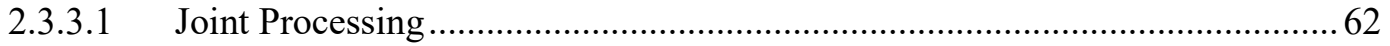

2.3.3.2 Coordinated Scheduling/Coordinated Beamforming $(\mathrm{CS} / \mathrm{CB})$........................ 62

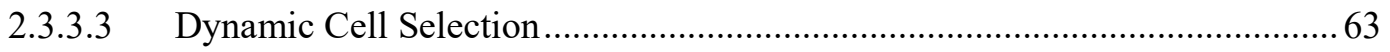

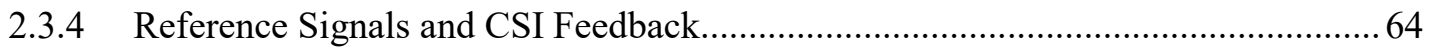

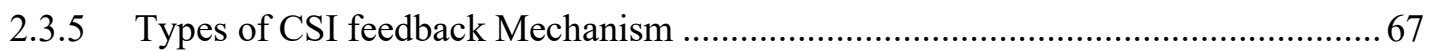

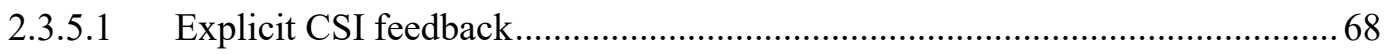

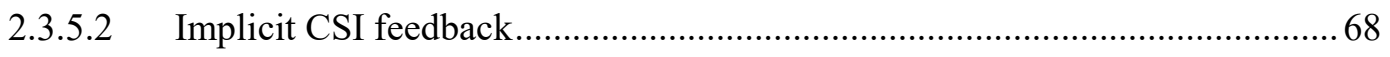

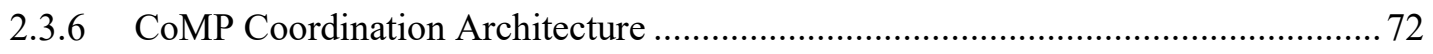

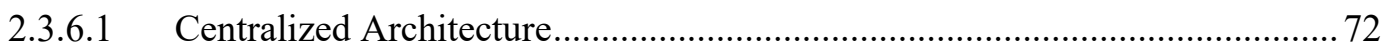

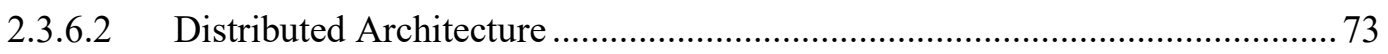

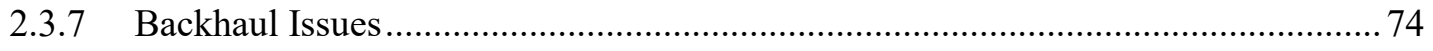

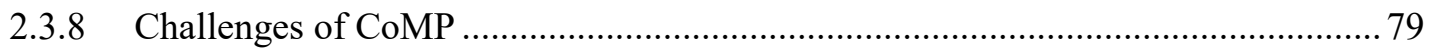

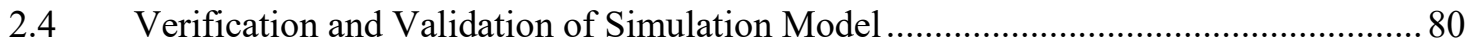

\section{Chapter: Coordinated Multi-Point Communication Using Dynamic}

Coordination Station .............................................................................................................. 86

3.1 DCEC for Homogeneous Cellular Networks ….................................................... 89

3.1.1 Coordination Station Election Algorithm for DCEC …..................................... 90

3.1.2 Signaling Procedure for DCEC in Homogeneous Cellular Networks .....................91

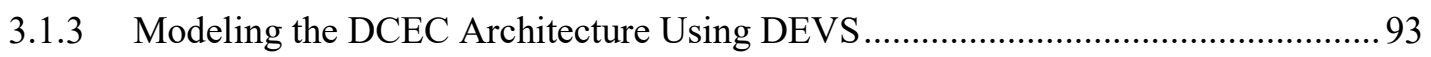

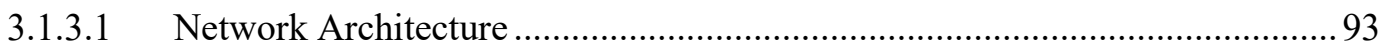




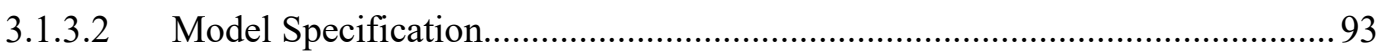

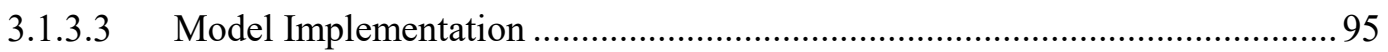

3.2 DCEC for Dense Heterogeneous Cellular Networks............................................... 97

3.2.1 Coordination Station Election Algorithm for DCEC-HetNets............................... 98

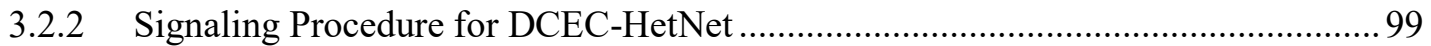

3.2.3 Modeling the DCEC-HetNet Coordination Architecture Using DEVS ................. 101

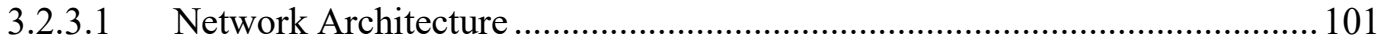

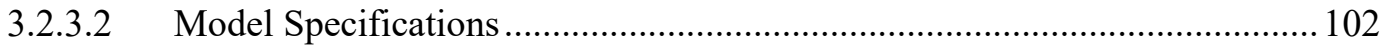

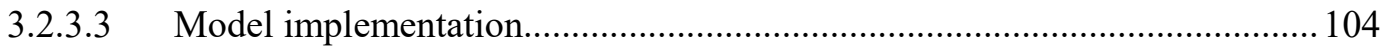

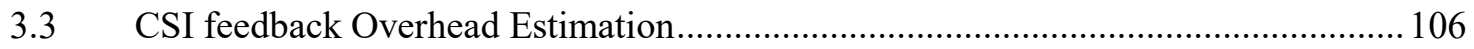

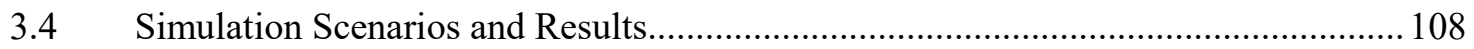

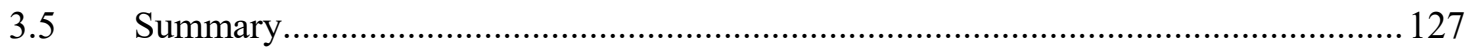

\section{Chapter: Handover Enhancement for Dense Heterogeneous Cellular Networks}

4.1 Handover Process in Wireless Cellular Networks................................................... 131

4.2 Enhanced Handover for Low and Moderate Speed UEs ......................................... 135

4.3 Modeling Handover and Oscillation in cellular networks ....................................... 138

4.4 Modeling the EHoLM in HetNets using DEVS …................................................ 140

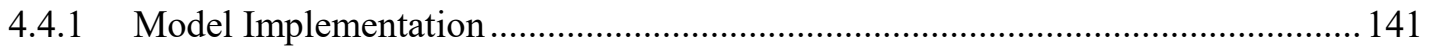

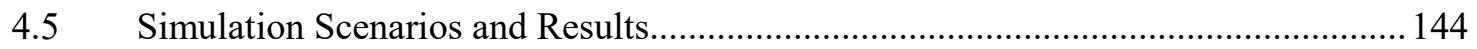

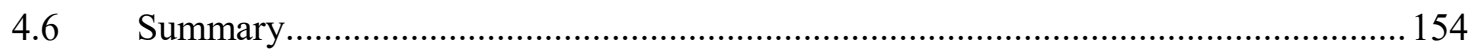

5 Chapter: Verification and Validation of Simulation Models................................ 156

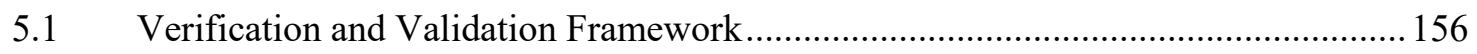

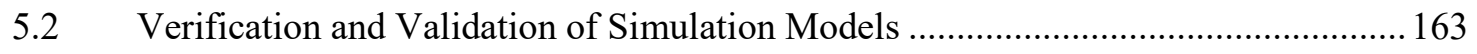

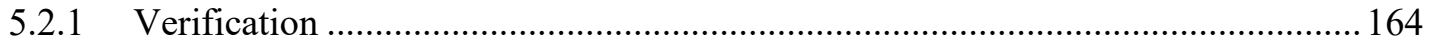

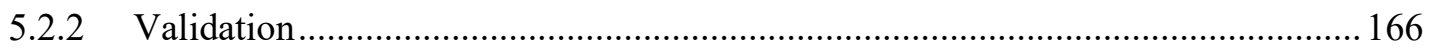


5.3 Summary.

6 Chapter: Conclusion and Future Work........................................................ 175

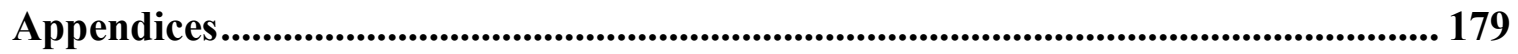

Appendix A Control Plane Protocol Overview ................................................................. 179

Appendix B Basic Transmission Scheme and Physical Resources ..................................... 181

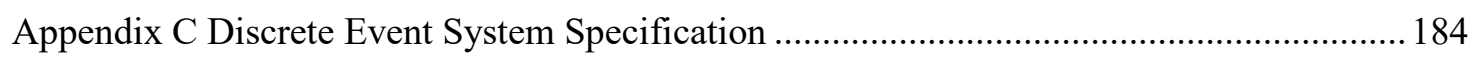

Bibliography ..................................................................................................................... 187 


\section{List of Tables}

Table 1: Key features for different generations ( $1 \mathrm{G}$ to $4 \mathrm{G})$ of cellular networks............ 40

Table 2: Major related works in ultra-dense heterogeneous networks .......................... 48

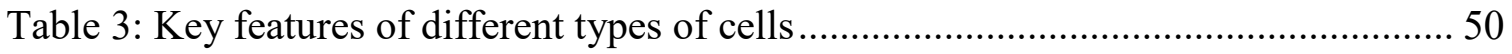

Table 4: Number of access points per $\mathrm{km}^{2}$ in UDHetNet ............................................ 53

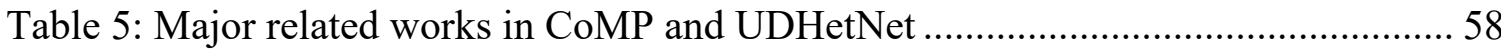

Table 6: Subband size according to system bandwidth for Subband level feedback ....... 70

Table 7: Subband size and corresponding selected number of subbands according to the

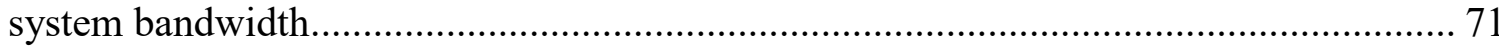

Table 8: Performance evaluation of different CoMP schemes with varying CSI feedback

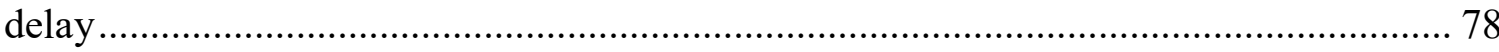

Table 9: Number of bits in RI according to the antenna ports ................................... 108

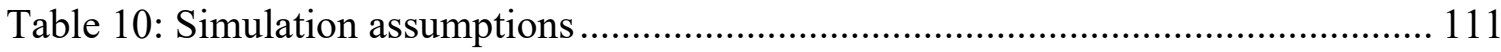

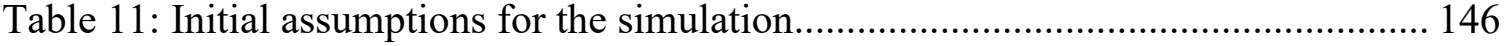

Table 12: Simulation assumption and corresponding references ............................... 167

Table 14: Resource parameters of LTE and beyond cellular networks ....................... 183 


\section{List of Figures}

Figure 1: Overall architecture of cellular networks current and future ........................... 36

Figure 2: $5 \mathrm{G}$ requirements, enabling technologies and future applications...................... 43

Figure 3: A simplified general architecture of 5G wireless cellular access networks ...... 45

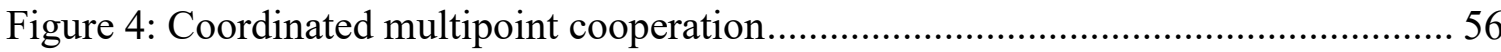

Figure 5: 3GPP CoMP scenarios …………………..................................................... 59

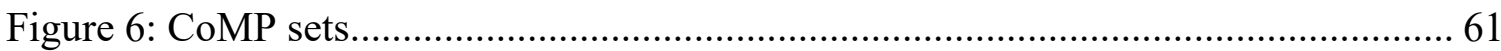

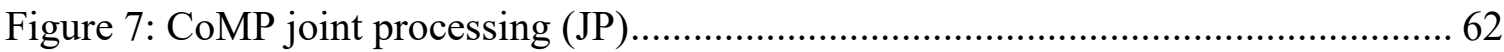

Figure 8: Coordinated scheduling/coordinated beamforming $(\mathrm{CS} / \mathrm{CB})$.......................... 63

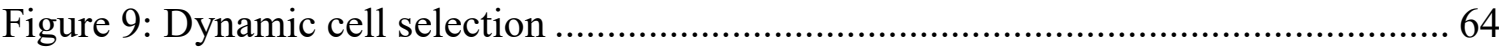

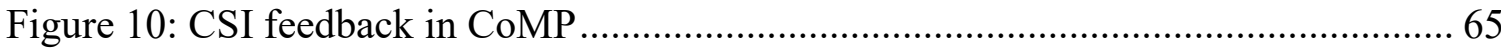

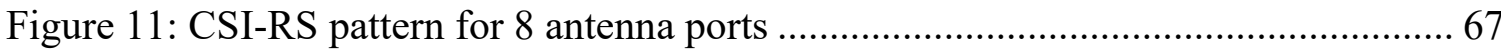

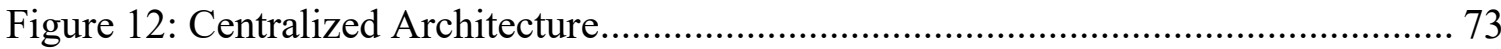

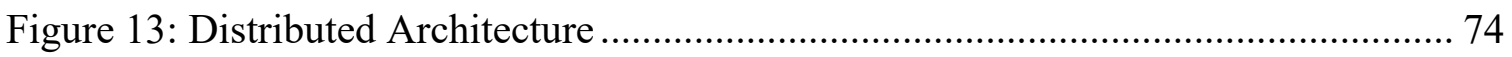

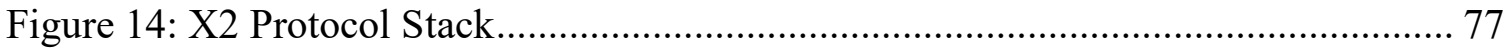

Figure 15: Simplified view of the DCEC CoMP coordination architecture ...................... 88

Figure 16: Message transfer to establish CoMP with CS election in DCEC..................... 92

Figure 17: Homogeneous Cellular Network Architecture ............................................... 93

Figure 18: Simplified DEVS model for CoMP coordination architecture in homogeneous

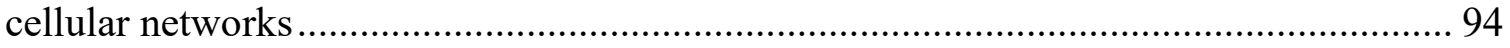

Figure 19 : Simplified class diagram of the model for homogeneous networks .............. 96

Figure 20: Message transfer to elect CS and establish CoMP in DCEC-HetNet ........... 101 
Figure 21: Simplified Network Architecture for HetNets 102

Figure 22: Simplified DEVS model for CoMP coordination architecture in heterogeneous cellular networks 103

Figure 23: Simplified class diagram of the model 105

Figure 24: Simulation scenarios considered to evaluate the DCEC and DCEC-HetNet coordination architecture 110 Figure 25: Number of control messages at different time intervals for DCEC, Centralized and Distributed architectures: messages over the backhaul and UE to MeNB. 114 Figure 26: Cumulative control messages for DCEC without CS change, DCEC with CS change every 1s/100ms, Centralized, and Distributed Architectures 116 Figure 27: Cumulative number of messages for Centralized, DCEC and Distributed based on the density of the active UEs into CoMP in macro only networks 118 Figure 28: Non-Accumulative number of messages for bursty arrival of UEs with density of (a) $2 / \mathrm{km}^{2}$, (b) $4 / \mathrm{km}^{2}$, (c) $9 / \mathrm{km}^{2}$ 120 Figure 29: DCEC-HetNet, Centralized, and Distributed architectures based on the number of control messages in the network with respect to the number of UEs in $700 \mathrm{~ms}$ 121 Figure 30: DCEC-HetNet, Centralized and Distributed based on the number of messages traveled in UEs to BSs and the Backhaul. Each three bars group represents 10ms (detailed view on the top left) 122

Figure 31: Aggregate number of control messages for Centralized, DCEC-HetNet and Distributed based on the density of the active UEs into CoMP cooperation in heterogeneous networks 124 
Figure 32: Number of control messages per second for DCEC-HetNet, Centralized and Distributed CoMP coordination architecture based on the density of the active UEs into in heterogeneous networks 125

Figure 33: Signaling overhead in DCEC-HetNet, Centralized and Distributed CoMP coordination architecture with respect to different CSI feedback schemes for 100 UEs 126 Figure 34: Average feedback delay for 50, 100, and 200 UEs in the network 127

Figure 35: A simplified view of ultra-dense heterogeneous networks and handover..... 129

Figure 36: Densification and its impact in the networks .......................................... 130

Figure 37: Simplified handover process for LTE and LTE-A cellular networks ........... 133

Figure 38: Simplified view of the EHoLM handover scheme ..................................... 136

Figure 39: EHoLM handover scheme flowchart ....................................................... 138

Figure 40: Handover oscillation modeling and computing the number of handovers and handover oscillations 139

Figure 41: Simplified DEVS model for EHoLM in heterogeneous networks 141

Figure 42: Simplified class diagram of the DEVS model for EHoLM handover scheme 143

Figure 43: Simplified simulation scenarios with MeNB and PeNB placement............... 145

Figure 44: Number of handovers with respect to the number of UEs ........................... 148

Figure 45: Number of handovers with respect to UE speed ....................................... 150

Figure 46: Number of handovers with respect to each of the UEs ................................. 151

Figure 47: Number of handovers in EHoLM and Conventional handover schemes with

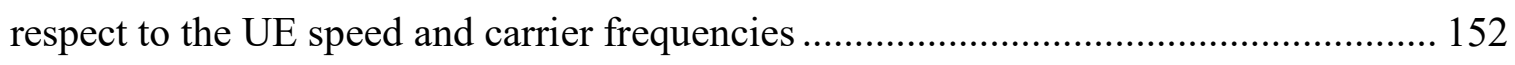

Figure 48: Number of handover oscillations with respect to the UE speed.................... 153 
Figure 49: Percentage of UEs do not participate in the handover process

Figure 50: A common framework for verification and validation process

Figure 51: Sample code snippet of the simulation model (atomic models) developed from the DEVS model for handover process in heterogeneous cellular networks..... 161

Figure 52: Sample code snippet of the simulation model (coupled models) developed from the DEVS model for handover process in heterogeneous cellular networks 162 Figure 53: Comparison of mathematical model and simulation model for all of the three architectures 171

Figure 54: Number of handovers with respect to UE speed for homogeneous networks and heterogeneous networks. 173

Figure 55: Control plane protocols 179

Figure 56: Transmission scheme and physical resource elements in wireless networks 182 Figure 57: A simple DEVS coupled model with two atomic models 186 


\section{List of Equations}

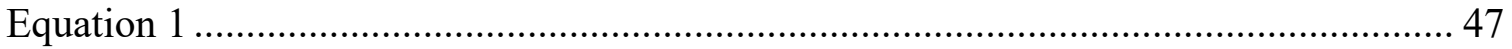

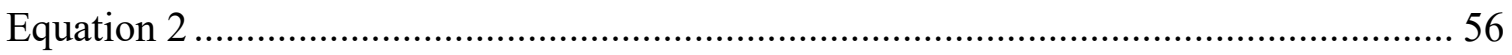

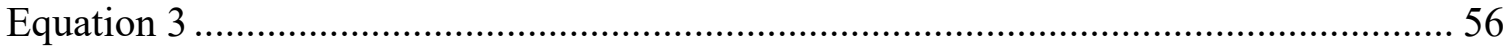

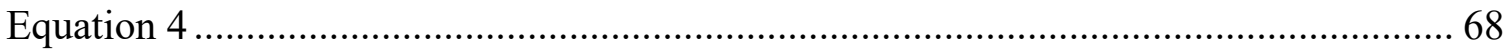

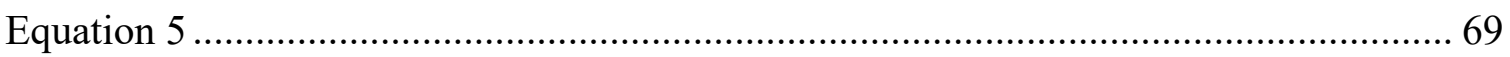

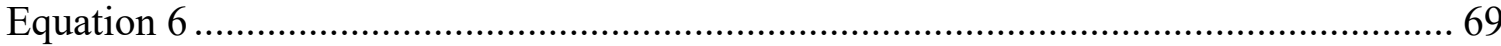

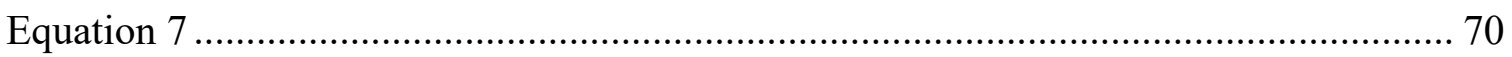

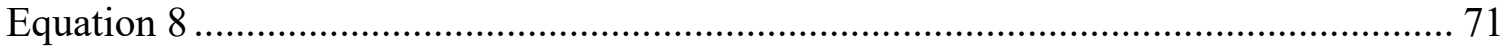

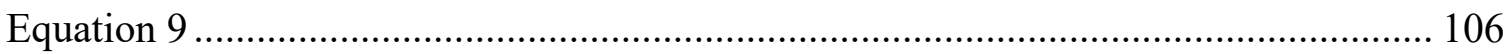

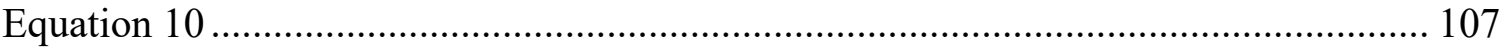

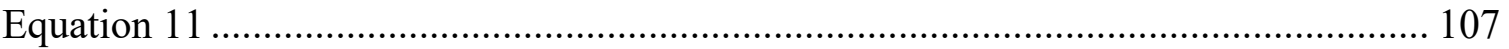

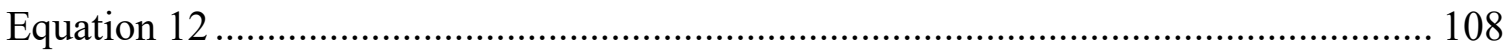

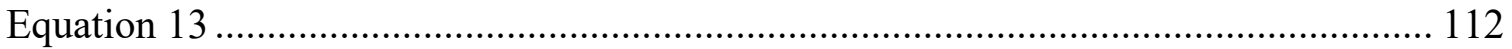

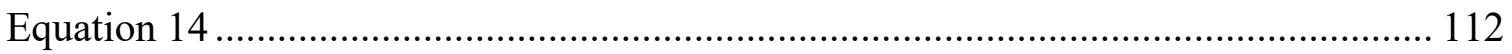

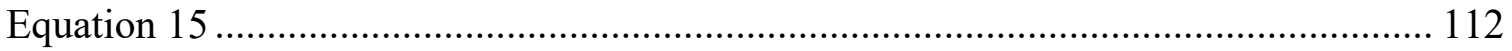

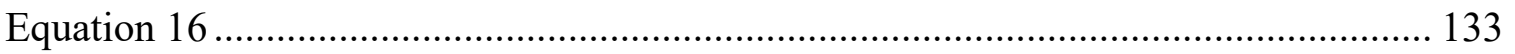

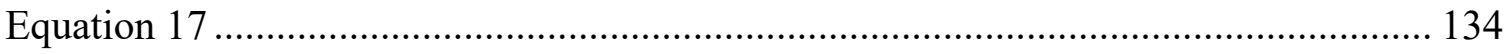

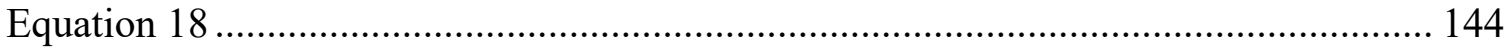

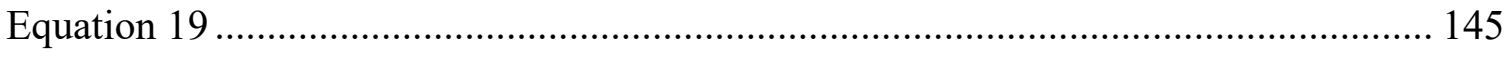

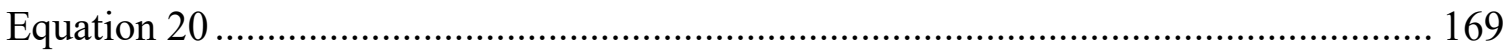

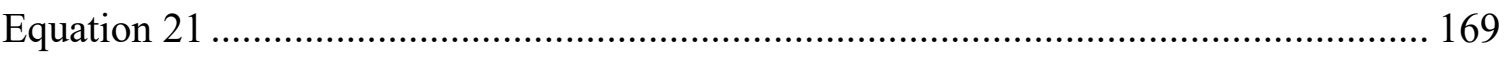

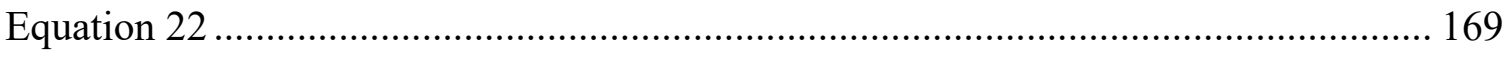


Equation 23

Equation 24 ... 


\section{List of Acronyms}

$1 \mathrm{G} \quad$ First Generation

2G Second Generation

3G Third Generation

3GPP $\quad 3^{\text {rd }}$ Generation Partnership Project

4G Fourth Generation

5G Fifth Generation

$5 \mathrm{GC} \quad 5 \mathrm{G}$ Core Network

AMPS Advanced Mobile Phone System

BBU Baseband Unit

BS Base Station

BW Bandwidth

CoMP Coordinated Multipoint

CQI Channel Quality Indicator

CS Coordination Station

$\mathrm{CS} / \mathrm{CB} \quad$ Coordinated Scheduling/Coordinated Beamforming

CSI Channel State Information

CSI-RS Channel State Information Reference Signal

CU Central Unit

DCEC Direct CSI-feedback to Elected Coordination-station

DCEC-HetNet Direct CSI-feedback to Elected Coordination-station for Heterogeneous Networks

DCS Dynamic Cell Selection 


$\begin{array}{ll}\text { DEVS } & \text { Discrete Event system Specifications } \\ \text { DM-RS } & \text { Demodulation Reference Signals } \\ \text { EDGE } & \text { Enhanced Data GSM Environment } \\ \text { EHoLM } & \text { Enhanced Handover for Low and Moderate speed UEs } \\ \text { eNB } & \text { Evolved Node B } \\ \text { EPC } & \text { Evolve Packet Core } \\ \text { E-UTRAN } & \text { Evolve Universal Terrestrial Radio Access Network) } \\ \text { FDMA } & \text { Frequency Division Multiple Access } \\ \text { FM } & \text { Frequency Modulation } \\ \text { GMSK } & \text { Gaussian Minimum Shift Keying } \\ \text { GSM } & \text { Global System for Mobile communications } \\ \text { HeNB } & \text { Home eNB } \\ \text { HetNets } & \text { Heterogeneous Cellular Networks } \\ \text { HO } & \text { Handover } \\ \text { HOF } & \text { Handover Failure } \\ \text { HSPA } & \text { High Speed Packet Access (HSPA) } \\ \text { ICIC } & \text { Intercell Interference Coordination } \\ \text { ISD } & \text { Inter Site Distance } \\ \text { JT } & \text { Joint Transmission } \\ \text { JTACS } & \text { Japan Total Access Communication System } \\ \text { LTE } & \text { Long Term Evolution } \\ \text { LTE-A } & \text { Long Term Evolution Advanced } \\ \text { Moding and Simulation }\end{array}$




\begin{tabular}{ll} 
MAC & Medium Access Control \\
MeNB & Macro Evolved Node B \\
MIMO & Multiple Input Multiple Output \\
MMH & Macro to Macro Handover \\
MPH & Macro to Pico Handover \\
MR & Measurement Report \\
multi-RAT & Multiple Radio Access Technology \\
NAS & Non-Access Stratum \\
NG-RAN & Next Generation Radio Access Network \\
NMT & Nordic Mobile Telephone \\
OFDM & Orthogonal Frequency-Division Multiplexing \\
PDCP & Packet Data Convergence Protocol \\
PeNB & Pico eNB \\
PMH & Pico to Macro Handover \\
PMI & Precoding Matrix Indicator \\
PPH & Pico to Pico Handover \\
RB & Resource Block \\
RI & Rank Indicator \\
RLC & Radio Link Control \\
RRC & Radio Resource Control \\
RRH & Remote Radio Head \\
RRM & Radio Resource Management \\
RSR & Reference Signal Received Power \\
\hline
\end{tabular}


SINR

TACS

TDMA

TTT

UDN

UDHetNet

WCDMA
Signal to Noise plus Interference Ratio

Total Access Communication System

Time Division Multiple Access

Time To Trigger

Ultra Dense Network

Ultra Dense Heterogeneous Networks

Wideband Code Division Multiple Access 


\section{List of Symbols}

$\begin{array}{ll}B W_{\mathrm{nm}} & \text { Bandwidth allocated to } \mathrm{UE}_{\mathrm{n}} \text { connected to } \mathrm{NB}_{\mathrm{m}} \\ N_{F F T} & \text { Number of samples of fast Fourier transformation } \\ N_{R B}^{S B} & \text { Number of resource blocks in system bandwidth } \\ N_{R B}^{S b a n d} & \text { Number of resource blocks in subband } \\ N_{S C}^{R B} & \text { Number of Subcarriers per resource block } \\ N_{S y m b}^{R B} & \text { Number of symbols per resource block } \\ N_{S C}^{S B} & \text { Number of subcarriers in system bandwidth } \\ N_{S y m b}^{S L} & \text { Number of symbols per slot } \\ O_{f b_{C Q I-W B}} & \text { Wideband CQI feedback overhead } \\ O_{f b_{C Q I-S B}} & \text { Subband level CQI feedback overhead } \\ O_{f b_{C Q I-B M}} & \text { UE selected best-M CQI feedback overhead } \\ O_{f b_{C Q I-F}} & \text { Full CQI feedback overhead } \\ O_{f b_{C S I-W B}} & \text { Wideband CSI feedback overhead } \\ O_{f b_{C S I-S B}} & \text { Subband level CSI feedback overhead } \\ O_{f b_{C S I-B M}} & \text { UE selected best-M CSI feedback overhead } \\ O_{f b_{C S I-F}} & \text { Full CSI feedback overhead } \\ T_{C S I-R S} & \text { CSI feedback transmission for kth UE } \\ \Delta \mathrm{f} & \text { Sume duration } \\ T_{f} & \text { Symbol duration } \\ & \end{array}$




\section{Chapter: Introduction}

Communications is an inherent need for human interactions. Guglielmo Marconi first demonstrated radio's ability to provide communication on the move in 1897. Since then wireless communications methods and services have evolved remarkably. The cellular networks or mobile networks provide wireless communication to the users located within the radio coverage of the system. The networks accommodate a large number of users, by breaking the coverage area down into many small areas called cells, within a limited frequency spectrum. Each cell has at least one fixed transceiver named Base Station (BS) or evolved Node B (eNB). Cellular networks started with voice communication services, allowing the users to talk using mobile phones. Gradually, with the development of cellular technology, it introduced new services and applications such as text messaging, data transmission and reception, real-time streaming, social networks, online gaming etc.

\subsection{Motivation}

Smart devices and the mobile internet have unveiled a new world with unbound possibilities. The telecommunication industry has witnessed an explosion in a wide range of applications and services such as video streaming, network gaming and social networking, these have become part of people's life. As a result, the number of mobile broadband users, the demand for data rates and the total volume of data traffic is increasing very fast. The number of mobile broadband subscriptions is growing globally by around $25 \%$ each year, and it is expected to reach 7.7 billion by 2021 [1]. The growth rate of mobile data traffic between the fourth quarter of 2016 to the fourth quarter of 2017 was about 55 percent and it is expected to reach 48.3 Exabytes per month by $2021[2,1]$. 
Moreover, the next generation of wireless cellular networks also considers potential use cases, such as autonomous vehicle control, smart cities, remote surgery and eHealth, tactile internet etc. Due to these issues, $5 \mathrm{G}$ networks are expected to support an enormous number of connected devices, high bandwidth, being ultra-high reliable, ultra-low latency, minimum signaling overhead, energy efficient and almost with $100 \%$ coverage [3, 4]. Therefore, to keep the user experience at a satisfactory level by achieving the above goals, we need to provide new cellular algorithms and technologies.

In this context, network densification such as ultra-dense networks (UDN) or ultra-dense heterogeneous networks (UDHetNet) and multi-cell cooperation are considered as the foundation to achieve the data traffic growth needed $[5,6,7]$. UDHetNets are comprised of different types of wireless access nodes with different capabilities. It consists of coexisting macrocells and low-power nodes such as remote radio head (RRH), pico eNB (PeNB) and home eNB (HeNB). We will discuss different types of nodes in Chapter 2 in details. These low power small cells can reduce the load of the macrocells, increase the network capacity and improve the user performance at the edge of the cells.

However, in ultra-dense networks, two technical challenges are inter-cell interference (ICI) coordination and mobility management due to the dense deployment of small cells, and the randomness of the network topology [8]. Mobility management or handover is essential to provide a seamless connection to the users in the move. The handover comes at the expense of system overhead. In HetNets, due to a large number of small cells and different types of backhauling, the number of handovers and handover failures will increase significantly. The increase in the number of handovers in HetNets compared to a macro only networks could be $120 \%-140 \%$, depending on the speed of the user equipment (UE) [9]. In 
UDHetNet, the number of handovers could be even higher depending on the density of the networks. Therefore, reducing the number of handovers and handover failure rate in UDHetNet is a challenge, which is one of the research goals of this thesis work. Intercell interference coordination (ICIC) is also of high importance [10]: as the number of cells increases, the total number of users located on the edge of the cells increases, and at the cells' edges, the users experience lower signal strength and higher interferences from the neighboring cells. Thus, this dense deployment of the networks needs advanced interference mitigation techniques in order to coordinate, cancel or exploit such interference.

In order to improve the performance of cellular networks by mitigating intercell interference, multi-cell cooperation or coordinated multipoint (CoMP) transmission and reception is considered as an effective method, especially for cell edge users $[11,12,13]$. The idea of CoMP is to evolve from the conventional single-cell multi-user system to a multi-cell multi-user system, so that the UEs close to the cell edge can be served by multiple base stations. In CoMP-enabled systems, the base stations (BS, also called evolved Node B - eNB) are grouped into CoMP cooperation set. The eNBs of each of the cooperation set exchange information among them, and they process signals and provide services to the users jointly. As a result, the UEs can receive their signals simultaneously from one or more transmission points in a coordinated or joint-processing method, which can improve data rate coverage and cell edge throughput $[14,15]$. Though the idea of CoMP was introduced in LTE-Advanced networks, it is also considered as a key feature for future dense cellular networks to improve the spectral efficiency, throughput and cell edge performance $[16,11]$. Moreover, $3 \mathrm{GPP}$ release 14 also included CoMP in the study 
item for further enhancement focusing on the future dense networks [12]. Therefore, although by now there has been some research done on CoMP for 3GPP LTE-Advanced networks as a new ICI management technology, it should be further investigated.

In a CoMP enabled network, the scheduler needs accurate and updated channel state information (CSI) for adaptive transmission, as well as appropriate radio resource management (RRM) $[14,17]$. In order to provide this information to the scheduler, the UEs estimate the CSI and report it to their serving eNB periodically. The coordinated eNBs exchange the received CSI and/or data among them providing services to the UE. Accordingly, the CSI feedbacks increase the signaling overhead into the networks and feedback latency significantly that requires high bandwidth backhaul $[14,15,18,19,20]$. Moreover, the networks may require additional control units and low-latency links among the collaborating eNBs known as infrastructural overhead, which might increase the network costs $[15,21,22]$. The overhead depends on the CoMP coordination architecture. According to the literature, there are two types of coordination architectures available: centralized and distributed $[21,22,23,19]$.

In the centralized architecture, a central unit is responsible for handling radio resource scheduling by processing the CSI feedback information from the UEs. This architecture suffers from signaling overhead and infrastructure overhead. It also increases the latency of the CSI feedback. In the distributed architecture, the coordinated cells exchange data and CSI over a fully meshed signaling network using X2 interfaces and a star-like S1 network. This architecture also increases the signaling overhead into the network. These signaling overhead and latency are the key causes for performance degradation of cooperative cellular networks $[22,11]$. Therefore, signaling overhead and CSI feedback 
latency reduction is another goal of this thesis work. We discussed details about the centralized and distributed architecture in Section 2.3.6. However, in a practical system, several challenges emerge, among which in this research we focus on CSI feedback overhead and latency, and mobility management. We discussed more detail about current research challenges of CoMP and UDHetNet in Chapter 2.

\subsection{Objectives}

The objective of the research is to develop a new coordination architecture in order to minimize the signaling overhead and feedback latency, and enhance the handover process for cooperative communication that eventually will improve the performance of cellular networks. More precisely, this research aims to achieve the following objectives:

- Propose an efficient coordination architecture for coordinated multipoint (CoMP) communications for reducing signaling overhead and channel state information (CSI) feedback latency.

- Propose an enhanced algorithm for handover process in the next generation dense heterogeneous cellular networks to minimize the number of handovers, handover oscillation and handover failure rate.

- Develop simulation models for cellular networks employing the proposed algorithms using discrete event system specifications (DEVS).

- Evaluate the proposed methods to see how well the algorithms improve the performance of cellular networks by reducing the control plane load and latency within the system. 
We achieved our research objectives that are already patented and published in peerreviewed journals and conferences as presented in Section 1.4. There are potential opportunities to extend this work that will be discussed in the conclusion and future work chapter.

\subsection{Contributions}

In this thesis, we present a CoMP coordination architecture named Direct CSI-feedback to Elected Coordination-station (DCEC) $[24,25,26]$, with the aim of reducing the signaling overhead and latency of the CSI feedback, which eventually will increase the throughput of the cellular networks. In this architecture, one of the cooperating eNBs in the CoMP cooperation set, elected dynamically, will act as a coordination station (CS), and the UEs in the same CoMP cooperating set will send the CSI feedback to this CS only. Thereon, the CS will calculate the CSI information of all the participating UEs, determining the cooperating set, and will be in charge of scheduling. It should be noted that a cooperating set is a set of eNBs and RRHs, directly and/or indirectly participate in the transmission and reception process of a UE [27]. In Section 2.3.2, we discussed more details about CoMP sets. There will also be no additional hardware necessary for this solution. So, the costs for switching to such architecture should be small. We extended the coordination architecture for heterogeneous cellular networks named DCEC-HetNet. This research was carried out in collaboration with Ericsson Canada and the idea is already patented [24].

In order to analyze the performance of the DCEC architecture, we built simulation models and ran simulations of various scenarios suggested by the $3 \mathrm{GPP}$ specifications. The $3 \mathrm{GPP}$ mobile broadband standard proposed and agreed four different network scenarios for CoMP for further study [27, 28]. We discussed different types of scenario in Chapter 2. 
We built simulation model and ran simulations for two other conventional architectures as well: centralized and distributed, using discrete event system specifications (DEVS) formalism for different scenarios [29]. A very brief discussion about DEVS is presented in Appendix C. DEVS is a powerful formal modeling and simulation methodology for discrete-event dynamic systems. Simulation results show that DCEC architecture reduces the number of control messages transmitted within the CoMP cooperation networks. Although it requires more control messages to elect the CS in the beginning, it outperforms the other two architectures as time advances. Furthermore, in DCEC architecture the CSI feedback does not need to travel through X2 or S1 links, which reduces the feedback latency as well. The details about the DCEC coordination architecture for cooperative cellular communication is presented in Chapter 3.

We also proposed a novel handover method named EHoLM: Enhanced Handover for Low and Moderate speed UEs for future generation dense heterogeneous cooperative cellular networks [30, 31]. In EHoLM, we use control plane and data plane separation for the UEs that are within the CoMP transmission and reception. The CoMP transmission reduces the inter-cell interference; hence, the signal quality of the serving cell remains better than the conventional transmission for a UE. Therefore, in the EHoLM handover, the handover criteria will not be satisfied until a UE moves from a CoMP to a no CoMP region of different eNB or to a different CoMP set without the current serving eNB instead of the conventional handover criteria (A3 event). The A3 event is discussed in Chapter 4. Simulation results also clearly show that the EHoLM handover method reduces the number of handovers and handover oscillations. The reduction of handover will improve the network performance as well as the reduction of handover failure rate will improve the user 
experience. The number of handovers, handover oscillations and the handover failure rate are the three key performance metrics to evaluate a handover process $[9,32]$.

To study the potential of the EHoLM handover procedure, we considered the HetNets scenarios suggested in $[9,32]$. We designed a DEVS simulation model to examine the performance of EHoLM in dense heterogeneous cellular networks. We ran a series of simulations on both EHoLM and the conventional handover process. The simulation results show that the proposed handover process significantly reduces the number of handovers and handover oscillations in heterogeneous cellular networks.

As we stated before, for modeling and simulation (M\&S) we used DEVS formalism and the $\mathrm{CD}++$ toolkit that implements DEVS theory $[33,34]$. We discussed very briefly about the DEVS in Appendix C. To ensure the credibility of the simulation results we should verify and validate the simulation model. In this thesis we provide a general understanding of how simulation model can be verified and validated. A revised lifecycle for modeling and simulation accommodating both conceptual and formal approaches of verification and validation $(\mathrm{V} \& \mathrm{~V})$ process has also been presented. Moreover, we also present how we verified and validated the simulation models we developed and used in our research.

\subsection{Related Publications}

The proposed coordination architecture and enhance handover process are published in peer-reviewed conference proceedings, journals and patented in collaboration with Ericsson Canada. In particular, the publications I have during the $\mathrm{PhD}$ study period related to my thesis are listed below based on the date of publication in a descending order. 


\section{Patent:}

[P1] Kazi, Baha Uddin, Gabriel Wainer, Gary Boudreau and Ronald Casselman, "Coordinated Multi-Point (CoMP) Method and Systems Using a Coordination Station," U.S. Patent P47112, 2015

\section{Journal articles:}

[J4] Kazi, Baha Uddin, and Gabriel Wainer. "Coordinated Multi-Cell Cooperation with User Centric Dynamic Coordination Station," Wireless Networks, Springer, 2018. (Submitted)

[J3] Tavanpour Misagh, Baha Uddin Kazi, Gabriel. Wainer, "Discrete Event Systems Specifications for Modeling and Simulation of Wireless Networking Applications," International Journal of Numerical Modeling: Electronic Networks, Device and Fields, John Wiley \& Sons, 2018. (Revisions submitted).

[J2] Kazi, Baha Uddin, and Gabriel Wainer. "Next Generation Wireless Cellular Networks: Ultra-Dense Multi-Tier and Multi-Cell Cooperation Perspective," Wireless Networks, Springer, 2018: 10.1007/s11276-018-1796-y.

[J1] Kazi, Baha Uddin and Gabriel Wainer. "Integrated cellular framework for modeling ecosystems: Theory and applications." SIMULATION: Transactions of The Society for Modeling and Simulation International, 2017: 0037549717706007.

\section{Conference articles:}

[C7] Kazi, Baha Uddin, and Gabriel Wainer. "Handover oscillation reduction in ultra-dense heterogeneous cellular networks using enhanced handover approach," 
In Proceedings of the 21st Communications and Networking Symposium (CNS2018), Society for Computer Simulation International, ACM, 2018.

[C6] Kazi, Baha Uddin, and Gabriel Wainer. "Handover enhancement for LTEAdvanced and beyond heterogeneous cellular networks," In Proceedings of International Symposium on Performance Evaluation of Computer and Telecommunication Systems (SPECTS-2017), pp. 1-8. IEEE, 2017.

[C5] Wainer, Gabriel, Mohammad Etemad, and Baha Uddin Kazi. "Modeling Coordinated Multipoint with a dynamic Coordination Station in LTE-A mobile network," In Proceedings of 14th IEEE International Conference on Networking, Sensing and Control (ICNSC-2017) pp. 807-812. IEEE, 2017.

[C4] Kazi, Baha Uddin, Gabriel Wainer, and Victor Guimaraes da Silva. "Modeling and simulation of user mobility and handover in LTE and beyond mobile networks using DEVS formalism," In Proceedings of the 20th Communications and Networking Symposium (CNS-2017), p. 1. Society for Computer Simulation International, ACM, 2017.

[C3] Kazi, Baha Uddin, and Gabriel Wainer. "Formal modeling and simulation to analyze the dynamics of malware propagation in networks using Cell-DEVS," In Proceedings of the 20th Communications \& Networking Symposium (CNS-2017), p. 6. Society for Computer Simulation International, ACM, 2017.

[C2] Kazi, Baha Uddin, Mohammad Etemad, Gabriel Wainer, and Gary Boudreau. "Signaling overhead and feedback delay reduction in heterogeneous 
multicell cooperative networks," In Proceedings of International Symposium on Performance Evaluation of Computer and Telecommunication Systems (SPECTS2016), pp. 1-8. IEEE, 2016.

[C1] Kazi, Baha Uddin, Mohammad Etemad, Gabriel Wainer, and Gary Boudreau. "Using elected coordination stations for CSI feedback on CoMP downlink transmissions," In Proceedings of International Symposium on Performance Evaluation of Computer and Telecommunication Systems (SPECTS2016), pp. 1-8. IEEE, 2016.

\subsection{Outline of The Thesis}

The rest of this thesis is organized as follows: in chapter 2 , the background of mobile networks and the different generations of mobile systems are discussed. In addition, we provide an extensive review on the related works that have been done in the same area (CoMP and UDHetNet) as the topic of this research. In Chapter 3, we presented the DCEC coordination architecture for multicell cooperative cellular networks. We discussed the DCEC approach in the context of both homogeneous and heterogeneous cellular networks. The EHoLM handover procedure is described in Chapter 4. In addition, in this chapter, simulation scenarios and results are also presented. The verification and validation $(\mathrm{V} \& \mathrm{~V})$ of the models are presented in Chapter 5. A common framework for both formal and conceptual V\&V process has also been presented in this chapter. Finally, we conclude in Chapter 6 with some potential future directions of the research work. 


\section{Chapter: Background and State of The Art}

The word "telecommunication" is derived from the Greek word "tele", meaning distance and the word "communicate", meaning sharing. Human being throughout the years communicated using different available technologies. As time passed, technology advanced gradually, and telecommunication networks are now reliable and efficient. Nowadays, telecommunication networks encompass mobile networks, fixed line networks and internet with different services and applications. In this thesis, we use mobile networks or cellular networks interchangeably.

In this chapter, we have made an extensive literature review on next generation $5 \mathrm{G}$ wireless communication focusing on ultra-dense heterogeneous networks (UDHetNets) and multicell cooperation. We first discuss the architecture and key technology enablers to achieve the goals of the 5G system discussed before. Subsequently, we discuss the stateof-the-art on UDHetNets and CoMP, and we give a categorization of the different methods. Finally, we discuss the major research challenges and open issues that require further investigation in this active area of research.

The overall architecture of LTE (long-term evolution) and LTE-Advanced cellular networks is shown in Figure 1(a) [35]. It consists of core networks or EPC (evolved packet core) and radio access networks or E-UTRAN (evolved universal terrestrial radio access network). The next generation 5G cellular networks architecture is shown in Figure 1(b). In this architecture, EPC is specified as $5 \mathrm{G}$ core network (5GC) and E-UTRAN is specified 
as next generation radio access network (NG-RAN) to embrace next-generation technologies with LTE.

(a) LTE and LTE-A Architecture

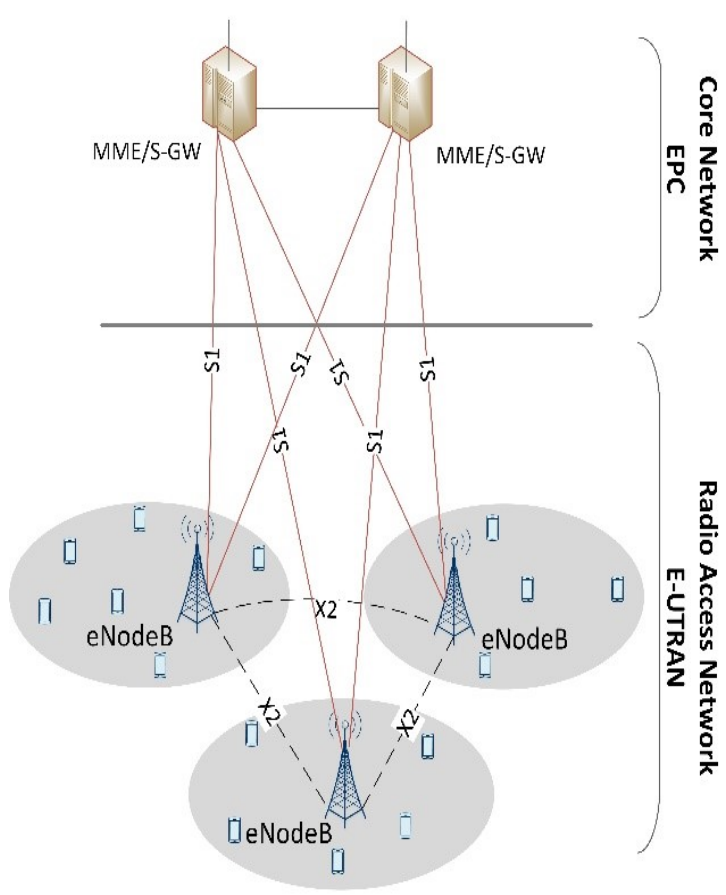

(b) Next Generation Architecture

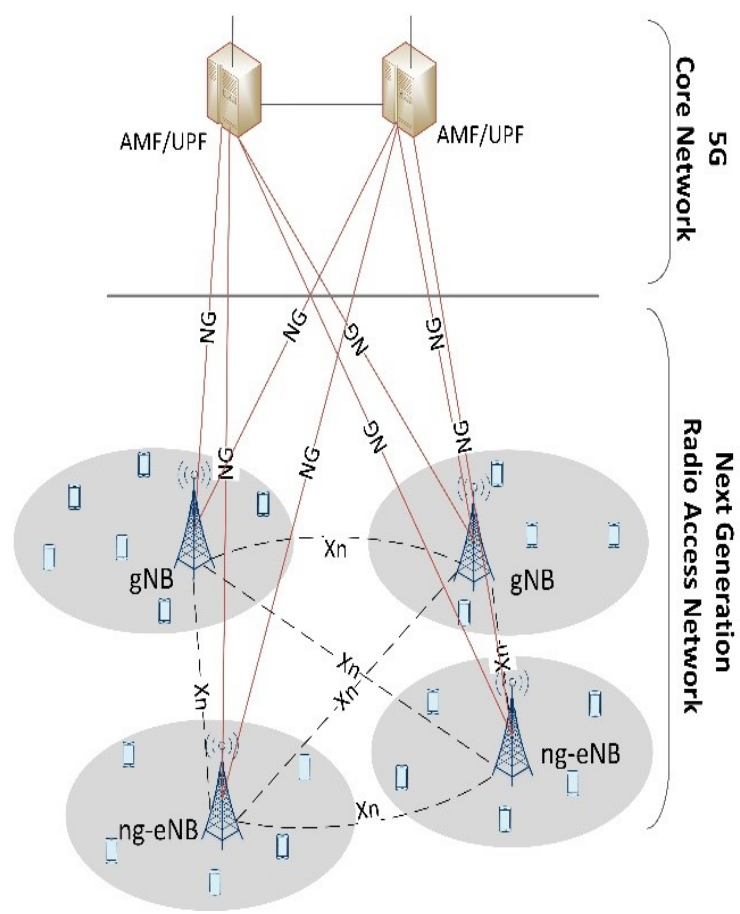

Figure 1: Overall architecture of cellular networks current and future

The major components in the LTE access network are eNBs (evolved Node B) and UEs (users' equipment). The eNBs or the base stations provide radio coverage over a geographical area and relay packets between the core network and mobile devices. The UEs, mobile devices within the coverage area are connecting to each other and to the eNBs by means of radio links. The eNBs are interconnected to each other through X2 interfaces. Moreover, the eNBs also connect to the EPC by means of S1 interfaces. In the next generation cellular networks, next-generation radio access networks (NG-RAN) consists of gNBs (next generation Node B), ng-eNBs (next generation evolved Node B) and UEs. The gNB provides new radio (NR) user plane and control plane termination towards the 
UE. The ng-eNB provides E-UTRAN user plane and control plane termination towards the UE. The gNB and the ng-eNB are interconnected by means of Xn interface. The gNB and the ng-eNB connect to the AMF (access and mobility management function) through the NG-C interface and to the UPF (user plane function) through the NG-U interface [36]. Finally, the core network connects access network to the wired Internet or the public telephony systems.

The advances in wireless technologies and radio spectral efficiency as well as the demand for data rate, mobility and coverage lead to the development of the cellular networks. History has shown that the cellular technology undergoes a major shift nearly every decade. In the following section, we discuss the evolution of cellular networks briefly.

\subsection{Evolution of Cellular Technologies}

Four generations of cellular technologies have been adopted up to now. A new generation has emerged approximately every decade roughly since 1980. A brief overview of the technological evolution of the cellular networks is as follows:

\subsubsection{First Generation}

The $1^{\text {st }}$ generation of cellular communication was introduced in the late 1970s. It was a basic analog system and it was designed for the voice communications. The frequency band used in the system was $800 \mathrm{MHz}$ and the data rate of the system was $2.4 \mathrm{kbps}$. These systems were based on circuit switching technology, which uses frequency modulation (FM), frequency division multiple accesses (FDMA) and a bandwidth (BW) of $30 \mathrm{kHz}$. Major subscribers of this generation of cellular networks were the advanced mobile phone 
system (AMPS) in North America, the total access communication system (TACS) in the United Kingdom, the Nordic mobile telephone (NMT) in Scandinavia, and the Japan total access communication system (JTACS). It has many disadvantages such as low quality, lack of security, limited subscribers, slow handover, poor battery life, while being limited to voice service. Moreover, the systems were incompatible to have a unified international standard $[3,37,38]$.

\subsubsection{Second Generation}

The $2^{\text {nd }}$ generation system was announced in the early 1990s. Digital technology was first introduced in this generation. The global system for mobile communications (GSM) was the first $2^{\text {nd }}$ generation cellular system with a data rate of up to $9.6 \mathrm{kbps}$. It used gaussian minimum shift keying (GMSK) modulation, time division multiple access (TDMA), and provided a bandwidth of $200 \mathrm{kHz}$. To improve the data rate of the $2^{\text {nd }}$ generation cellular networks, general packet radio services (GPRS) was introduced. GPRS uses packet switching technology and it is considered " $2.5 \mathrm{G}$ ". Subsequently, the enhanced data GSM environment (EDGE) provided a data rate of up to $200 \mathrm{Kbps}[38,39,3]$. The carrier frequencies (CF) used in this system was $850 \mathrm{MHz}, 900 \mathrm{MHz}, 1800 \mathrm{MHz}$ or $1900 \mathrm{MHz}$. This generation had some advantages over the $1^{\text {st }}$ generation such as enhanced services, improved security, better handset battery lifetime and most importantly, a unified international standard for mobile communication that prompted the growth of mobile 
communications worldwide. However, the $2^{\text {nd }}$ generation mobile phone suffered from low data rate, it could not handle complex data, a reduced network coverage etc. [3, 40].

\subsubsection{Third Generation}

The third generation (3G) of cellular systems was announced in early 2000. It introduced internet access and video and audio streaming capabilities. Moreover, global roaming and improved voice quality made the $3 \mathrm{G}$ systems widely used. It uses wideband code division multiple access (WCDMA) and high-speed packet access (HSPA) technologies to improve the performance. The $3^{\text {rd }}$ generation partnership project (3GPP), cellular standardization body released the evolved HSPA standard (known as HSPA+) in late 2008 for further improvement [3,38]. This generation of cellular systems uses frequency bands of $800 \mathrm{MHz}$, $850 \mathrm{MHz}, 900 \mathrm{MHz}, 1800 \mathrm{MHz}, 1900 \mathrm{MHz}$ or $2100 \mathrm{MHz}$. The $3 \mathrm{G}$ cellular systems use a bandwidth of $5 \mathrm{MHz}$ and improved data rate providing up to $30 \mathrm{Mbps}[37,41,39]$. It starts providing services combined mobile access with internet protocol (IP) based services.

\subsubsection{Fourth Generation}

The 3GPP introduced long-term evolution (LTE), a 4G system in the late 2010s. 4G is the current generation of cellular networks. The 4G networks use orthogonal frequencydivision multiplexing (OFDM) radio access technology and it supports transmission bandwidths of up to $20 \mathrm{MHz}$. The frequency bands used in this system is $1.8 \mathrm{GHz}$ and 2.6GHz. The intended peak data rate of LTE networks was up to $1 \mathrm{Gbps}$ while it achieves 300 Mbps [39]. However, the demand for data traffic is increasing rapidly. The global mobile data traffic will grow closely eightfold from 2015 to 2020, reaching 48.3 exabytes per month by 2021 from 3.7 exabytes in 2015, according to a recent CISCO report [42, 2]. 
Because of the demand for mobile data traffic, the 3GPP introduced LTE-Advanced cellular networks. The three main areas of research in LTE-Advanced include heterogeneous cellular networks (HetNets), enhanced spectral efficiency, and spectrum extension. A HetNet is comprised of different types of wireless access nodes with different capabilities such as macro, pico and femto nodes. We will discuss different types of nodes in the context of ultra-dense heterogeneous networks in Section 2.2.1. This approach is intended to improve network coverage and to increase the spectrum reuse. Coordinated multipoint (CoMP) transmission \& reception is another important scheme that improves the spectrum efficiency by mitigating the inter-cell interference (ICI) $[35,39,37]$. Table 1 summarizes the major features of the different generations (1G to $4 \mathrm{G}$ ) of cellular networks discussed.

Table 1: Key features for different generations (1G to $4 \mathrm{G})$ of cellular networks

\begin{tabular}{|c|c|c|c|c|}
\hline & & Gener & ations & \\
\hline & $I^{s t} G$ & $2^{\text {nd }} G$ & $3^{r d} G$ & $4^{\text {th }} G$ \\
\hline $\begin{array}{c}\text { Year } \\
\text { Introduced }\end{array}$ & Late 1970 s & Early 1990s & Early 2000 & Mid 2010s \\
\hline $\begin{array}{c}\text { Service } \\
\text { Technologies }\end{array}$ & Analog & Digital & Digital & Digital \\
\hline Switching & Circuit & Circuit / Packet & Packet & Packet \\
\hline Standards & $\begin{array}{l}\text { Advanced } \\
\text { Mobile Phone } \\
\text { System } \\
\text { (AMPS), } \\
\text { Total Access } \\
\text { Communication }\end{array}$ & $\begin{array}{l}\text { Global System } \\
\text { for Mobile } \\
\text { communications } \\
\text { (GSM), } \\
\text { General Packet } \\
\text { Radio Services }\end{array}$ & $\begin{array}{l}\text { Universal Mobile } \\
\text { Telecommunication } \\
\text { System (UMTS) }\end{array}$ & $\begin{array}{l}\text { Long Term } \\
\text { Evolution } \\
\text { (LTE) and } \\
\text { LTE- } \\
\text { Advanced }\end{array}$ \\
\hline
\end{tabular}




\begin{tabular}{|c|c|c|c|c|}
\hline & $\begin{array}{l}\text { System } \\
\text { (TACS), } \\
\text { Nordic Mobile } \\
\text { Telephone } \\
\text { (NMT) }\end{array}$ & $\begin{array}{l}\text { (GPRS), } \\
\text { Enhanced Data } \\
\text { GSM } \\
\text { Environment } \\
\text { (EDGE) }\end{array}$ & & \\
\hline $\begin{array}{c}\text { Access } \\
\text { Technologies }\end{array}$ & $\begin{array}{l}\text { Frequency } \\
\text { Division } \\
\text { Multiple } \\
\text { Accesses } \\
\text { (FDMA) }\end{array}$ & $\begin{array}{l}\text { Time Division } \\
\text { Multiple Access } \\
\text { (TDMA) and } \\
\text { Code Division } \\
\text { Multiple Access } \\
\text { (CDMA) }\end{array}$ & $\begin{array}{l}\text { Wideband Code } \\
\text { Division Multiple } \\
\text { Access (WCDMA), } \\
\text { Code Division } \\
\text { Multiple Access } \\
\text { (CDMA) 2000, } \\
\text { High-Speed Packet } \\
\text { Access (HSPA) and } \\
\text { HSPA+ }\end{array}$ & $\begin{array}{l}\text { Orthogonal } \\
\text { Frequency } \\
\text { Division } \\
\text { Multiplexing } \\
\text { (OFDM) }\end{array}$ \\
\hline $\begin{array}{l}\text { Carrier } \\
\text { Frequency }\end{array}$ & $800 \mathrm{MHz}$ & $\begin{array}{l}850 \mathrm{MHz}, \\
900 \mathrm{MHz}, \\
1800 \mathrm{MHz} \text { and } \\
1900 \mathrm{MHz} .\end{array}$ & $\begin{array}{l}800 \mathrm{MHz}, 850 \mathrm{MHz}, \\
900 \mathrm{MHz}, \\
1800 \mathrm{MHz}, \\
1900 \mathrm{MHz} \text { and } \\
2100 \mathrm{MHz}\end{array}$ & $\begin{array}{l}1.8 \mathrm{GHz} \text { and } \\
2.6 \mathrm{GHz}\end{array}$ \\
\hline Bandwidth & $30 \mathrm{KHz}$ & $200 \mathrm{kHz}$ & $5 \mathrm{MHz}$ & $20 \mathrm{MHz}$ \\
\hline Data Rate & $2.4 \mathrm{kbps}$ & $10-200 \mathrm{kbps}$ & 0.3-30 Mbps & 0.7-1 Gbps \\
\hline Applications & Voice & Voice and Data & $\begin{array}{l}\text { Voice, Data, Video } \\
\text { call, Mobile TV } \\
\text { etc. }\end{array}$ & $\begin{array}{l}\text { Voice, Data, } \\
\text { Video call, } \\
\text { Mobile TV, } \\
\text { Online }\end{array}$ \\
\hline
\end{tabular}




\begin{tabular}{|l|l|l|l|}
\hline & & gaming, \\
& & & Video \\
streaming \\
etc.
\end{tabular}

However, the number of mobile subscribers increases every day, the demand for the data rates doubled every year and new bandwidth-hungry \& low latency applications and services are introduced often $[1,6]$. These are the factors that are considering the major drivers towards a new generation such as the $5 \mathrm{G}$ systems.

\subsubsection{Next Generation Wireless Cellular Networks}

The fifth generation $(5 \mathrm{G})$ cellular networks have received significant attention from both academia and industry, as they are intended to overcome the challenges of existing cellular systems, such as the exponential growth of data traffic, coverage, lower latency, energy consumption, reliability, and cost. Merging the different research works by academia and industries, the aim of the next generation $5 \mathrm{G}$ networks is to provide approximately a system capacity of 1000 times higher, 10 times the data rates, 25 times the average cell throughput, 5 times reduced latency and 10 times longer battery life compared to the $4 \mathrm{G}$ networks [43, $44,40,45,46,47]$.

The $5 \mathrm{G}$ requirements and vision are derived from a set of requirements and potential use cases set by several industries and research bodies. For example, autonomous vehicle control enables driverless cars, which can improve traffic safety, increase productivity, and so on. Remote surgery and eHealth will provide us remote health monitoring such as electrocardiography (ECG), blood pressure, blood glucose and surgery for disaster 
response. In case of remote surgery, it is crucial for the surgeon to get the correct control and feedback with very strict requirements in terms of latency, reliability, and security. Moreover, smart cities will need remote monitoring of real-time traffic system, public safety, pollution, etc. The aggregation of all of these services leads to a very high density of interconnected devices with distinct characteristics in a communication framework. Figure 2 summarizes the key enablers, challenges, expected values, and some promising applications of the next generation of wireless cellular networks [45, 48, 47].

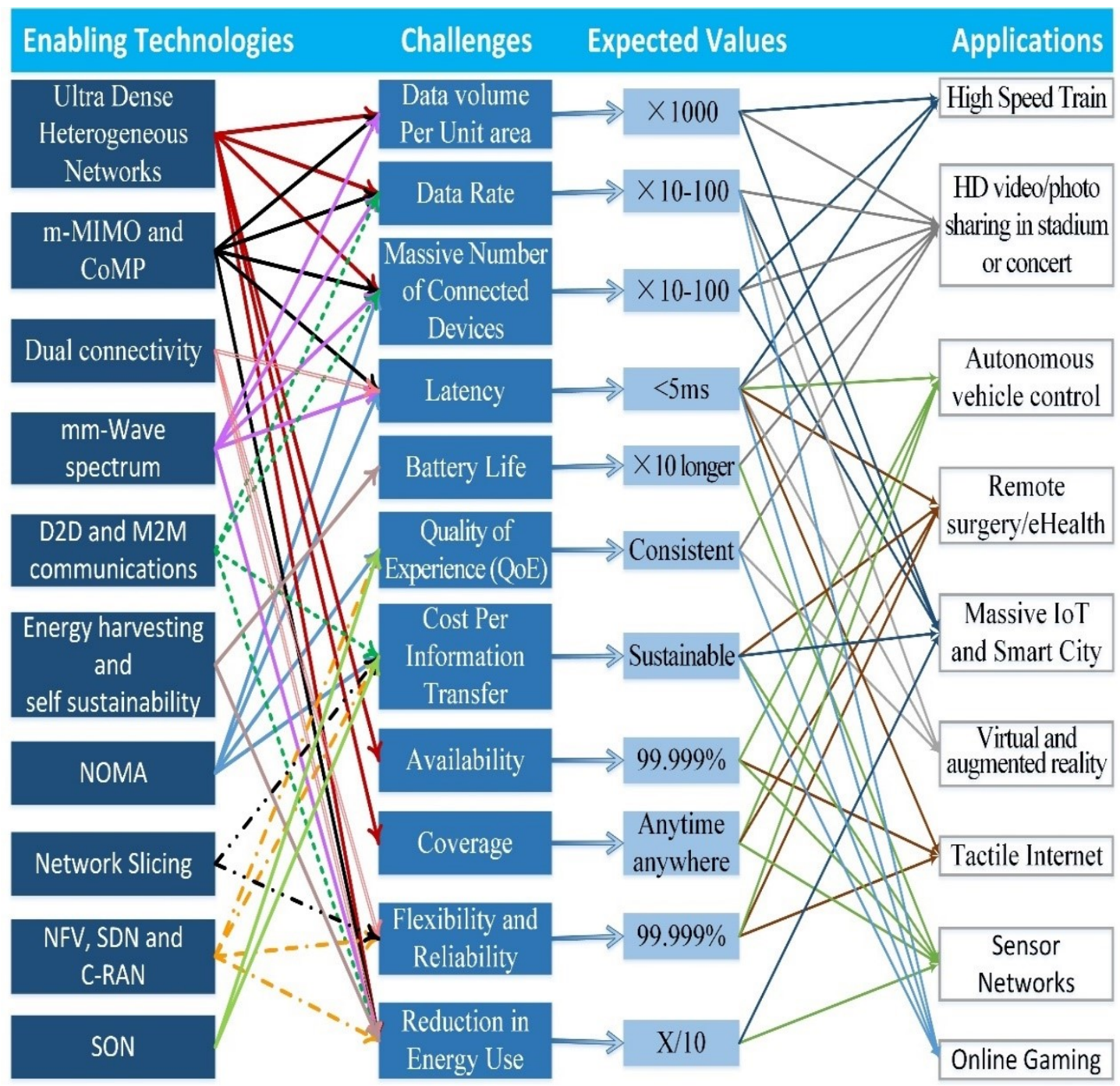

Figure 2: 5G requirements, enabling technologies and future applications 
To achieve these goals, the $5 \mathrm{G}$ cellular networks will adopt a set of new technologies. In the next subsection, we briefly discussed different key technology enablers adopted.

\subsubsection{5G Key Technology Enablers}

As discussed in the earlier section, it is unlikely that one technology enabler will be able to fit all use cases and applications. Therefore, based on several research results, different promising concepts have been identified. Ultra-dense network (UDN) or ultra-dense HetNet (UDHetNet), nonorthogonal multiple access (NOMA), massive multiple inputs multiple outputs (Massive-MIMO), coordinated multi-point (CoMP) communication, device to device (D2D) and machine to machine (M2M) communication, millimeter wave (mm-Wave) communication, energy harvesting, software-defined networking (SDN), network function virtualization (NFV) and cloud RAN (C-RAN) are the key enablers for next-generation cellular networks. However, the key focus of the access network enablers is to improve the system bandwidth, spectral efficiency, and coverage. Figure 3 shows a simplified general architecture of the next generation cellular access networks. 


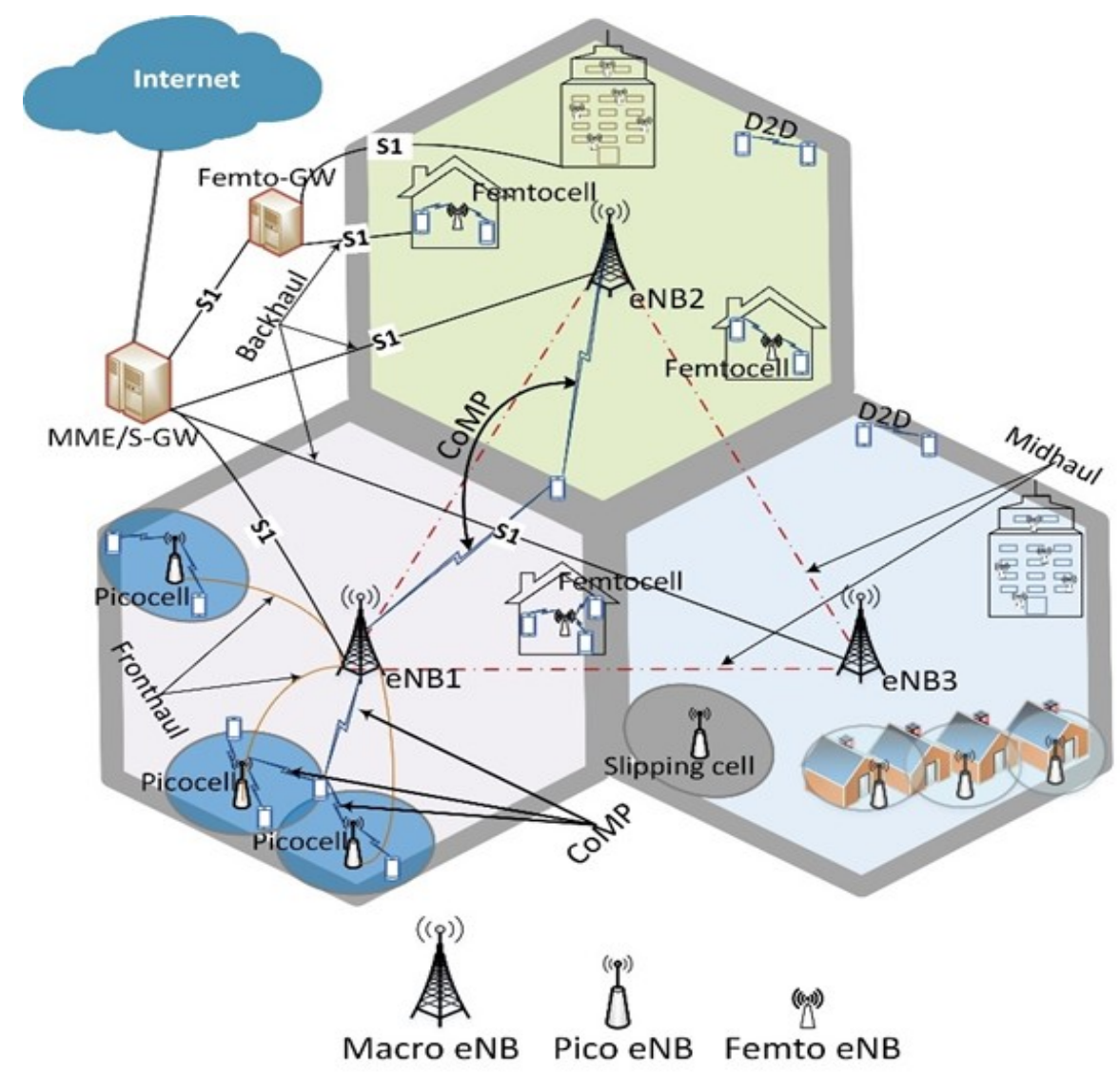

Figure 3: A simplified general architecture of $5 \mathrm{G}$ wireless cellular access networks

The ultra-dense heterogeneous network (UDHetNet) is one of the leading enablers, which is considered the foundation of 1000 fold data traffic growth $[5,6,7]$. It is a multi-tier network that includes legacy high-power macro cells and very dense low power small cells such as picocells, femtocells, relays and RRHs. The basic idea of UDN is to densify the access nodes in per unit area that increases the reusability of the spectrum and makes the access nodes closer to the UEs. The proximity of eNBs in dense networks increase the celledge area significantly, where UEs experience poor SINR. Consequently, interference mitigation is extremely important in UDHetNets. We will discuss UDHetNet in Section 2.2 in details. The coordinated multipoint (CoMP) operation can construct large 
cooperative multiple inputs multiple outputs transmission to avoid inter-cell interference, thus improving the UEs' SINR. The CoMP is considered a very effective technique to improve the coverage of high data rate, cell-edge throughput as well as system throughput. Though CoMP was introduced in LTE-A, it is also considered as a key feature for future dense cellular networks $[16,11,12]$. Therefore, CoMP combined with UDHetNet will play a vital role in improving coverage, energy efficiency, spectral efficiency and throughput of the next generation of cellular networks $[49,3,16]$. However, channel state information (CSI), associated with a large number of eNB antennas and coordination among multiple eNBs induce a huge amount of information exchange overhead into the networks. As a result, managing CSI is also a vital issue to achieve the gain of multi-antenna systems. Thus, multicell cooperation requires enhancements in the context of dense networks and our research in this thesis is focused on that. In the next two sections, we discuss UDHetNets and CoMP in details, including with the challenges that need to be investigated.

\subsection{Ultra-Dense Heterogeneous Networks}

The idea of UDHetNets is to have a very dense deployment of small cells combined with legacy macro cells. It is a multi-tier network. The distance between UEs and eNBs become shorter, spectrum reuse increase, and transmission power reduce. As a result, three primary gains of UDHetNets are: improved link quality, energy efficiency and capacity 
improvement. In order to better understand how the capacity of the network significantly improve, the network capacity can be defined as follows based on the Shannon theory [50].

$$
C=\sum_{e N B_{1}}^{e N B_{M}} \sum_{U E_{1 m}}^{U E_{n m}} B W_{n m} \log _{2}\left(1+S I N R_{n m}\right), \quad \text { Equation } 1
$$

where, $\left\{\mathrm{eNB}_{1} \ldots \mathrm{eNB} \mathrm{e}_{\mathrm{M}}\right\}$ is the set of eNBs deployed in the networks, $\left\{\mathrm{UE}_{1 \mathrm{~m}} \ldots \mathrm{UE}_{\mathrm{nm}}\right\}$ is the set of UEs connected to the $\mathrm{eNB}_{\mathrm{m}}$ and $\mathrm{m}=\{1 \ldots \mathrm{M}\} . B W$ is the total available bandwidth and $B W_{\mathrm{nm}}$ is the bandwidth allocated to $\mathrm{UE}_{\mathrm{n}}$ connected to $\mathrm{NB}_{\mathrm{m}}$. The $\mathrm{SINR}_{\mathrm{nm}}$ represents the quality of the signal experienced by the $\mathrm{UE}_{\mathrm{n}}$ connected to $\mathrm{eNB}_{\mathrm{m}}$.

The network densification increases the number of eNBs into the network that linearly increases the reusability of available $B W$, which eventually increase the capacity of the network. On the other side, cell densification reduces the cell size which results in the lower number of connected UEs to an eNB. Therefore, a larger $B W$ is available per UE. Moreover, as the cell size reduces, the average distance between a UE and the serving eNB reduces, which increase the quality of UE received signal. Table 2 summarizes the major research works with key points related to the UDHetNets. 
Table 2: Major related works in ultra-dense heterogeneous networks

\begin{tabular}{|c|c|c|}
\hline References & Work area & $\begin{array}{l}\text { Key points presented in the corresponding } \\
\text { referred articles }\end{array}$ \\
\hline $\begin{array}{c}{[50][51]} \\
{[52][7][6]} \\
{[53]}\end{array}$ & $\begin{array}{l}\text { UDHetNets } \\
\text { density }\end{array}$ & $\begin{array}{l}\text { - Analysis of the path loss model to study the } \\
\text { performance impact in small cell networks } \\
\text { (SCNs) } \\
\text { - UDHetNet capacity } \\
\text { - Network configuration in terms of density, } \\
\text { - Coverage probability }\end{array}$ \\
\hline $\begin{array}{c}{[54][55]} \\
{[56]}\end{array}$ & Mobility & $\begin{array}{l}\text { - Scheduling algorithm for UDN } \\
\text { - } \text { Frame structure for UDN } \\
\text { - User/Control plane separation } \\
\text { - Handover procedure for data only carrier }\end{array}$ \\
\hline $\begin{array}{l}{[57][50]} \\
{[7][58]}\end{array}$ & $\begin{array}{l}\text { Densification } \\
\text { challenges }\end{array}$ & $\begin{array}{l}\text { - Interference management } \\
\text { - } \text { Energy efficiency } \\
\text { - Backhaul } \\
\text { - Architecture }\end{array}$ \\
\hline $\begin{array}{l}{[59][60]} \\
{[61]}\end{array}$ & $\begin{array}{l}\text { Backhaul } \\
\text { distribution }\end{array}$ & $\begin{array}{l}\text { - Gateway based distribution architecture } \\
\text { - } \text { Backhaul energy efficiency } \\
\text { - } \text { mm-wave Backhaul } \\
\text { - } 5 \text { G backhaul architecture }\end{array}$ \\
\hline
\end{tabular}


In the following subsections, we provide a basic background of different types of cells considered for deployment in UDHetNets. We also discuss the fundamental features and architectures. Moreover, we present some future challenges and open issues of UDHetNets.

\subsubsection{Deployment of Cells}

Ultra-dense heterogeneous networks (UDHetNets) consist of various access technologies, each of which has different operating functions with different capabilities and constraints.

It enables efficient reuse of spectrum across the area of interest, which is one of the key solutions to achieve capacity increase for the next generation wireless cellular networks $[62,5,6]$. In general, in the UDHetNet, cells can be classified into three types. (a) fully functional high power macrocells (legacy cells); (b) fully functional small cells (picocells and femtocells), which are capable of performing all the functions of macrocells with low power in a smaller coverage area; and (c) macro extension access points, such as relays and remote radio heads (RRHs), which are the extension of the macrocell to extend the signal coverage without the baseband unit (BBU). Table 3 summarizes the features of different types of cells stated above $[10,59,63,64]$. 
Table 3: Key features of different types of cells

\begin{tabular}{|c|c|c|c|c|c|}
\hline $\begin{array}{l}\text { Types of } \\
\text { nodes }\end{array}$ & $\begin{array}{c}\text { Deployment } \\
\text { Scenario }\end{array}$ & $\begin{array}{l}\text { Transmit } \\
\text { Power }\end{array}$ & Coverage & Backhaul & Placement \\
\hline Macrocell & Outdoor & $\begin{array}{l}43-46 \\
\mathrm{dBm}\end{array}$ & Few km & $\begin{array}{c}\text { S1 } \\
\text { interface }\end{array}$ & Planned \\
\hline Picocell & Indoor/outdoor & $\begin{array}{l}23-30 \\
\mathrm{dBm}\end{array}$ & $<300 \mathrm{~m}$ & $\begin{array}{c}\text { X2 } \\
\text { interface }\end{array}$ & Planned \\
\hline Femtocell & Indoor & $<23 \mathrm{dBm}$ & $10-50 \mathrm{~m}$ & $\begin{array}{l}\text { Internet IP } \\
\text { (Non-ideal) }\end{array}$ & Unplanned \\
\hline Relays & Indoor/outdoor & $30 \mathrm{dBm}$ & $300 \mathrm{~m}$ & Wireless & Planned \\
\hline RRHs & Outdoor & $\geq 30 \mathrm{dBm}$ & $\begin{array}{l}300 \mathrm{~m}- \\
500 \mathrm{~m}\end{array}$ & $\begin{array}{l}\text { Fiber } \\
\text { (Ideal) }\end{array}$ & Planned \\
\hline
\end{tabular}

The details of the different cell types are discussed as follows:

- Macrocells consist of conventional operator installed outdoor eNBs. They are deployed in a planned manner, providing open public access and covering a wide area typically of a few kilometers. They are usually intended to provide a guaranteed minimum data rate under a maximum tolerable delay and outage constraints. Macro eNB (MeNB) typically transmit high power level such as 43-46 $\mathrm{dBm}$.

- Picocells consist of low power operator installed eNBs, named PeNB. They are typically deployed in outdoor and indoor by the provider in a planned manner. The 
transmit power range from $250 \mathrm{~mW}$ to $2 \mathrm{w}$ for outdoor and about $100 \mathrm{~mW}$ for indoor. However, picocells have the same access features and backhaul as macrocells to provide high bandwidth and low latency.

- Femtocells are usually deployed by users indoor (home, office, meeting room etc.). They are low power access points deployed in an unplanned manner with typical transmit power is $23 \mathrm{dBm}$ or less. They serve very few home users, where most of the data traffic generated as we discussed before. The backhaul network for femto eNBs (FeNB) is facilitated by the consumers' broadband connections such as digital subscriber line (DSL), cable or fiber. According to the access of a femtocell, it operates in three different modes: open, closed and hybrid. Closed femtocells are restricted to the closed subscriber group (CSG). In this case UEs cannot connect to the strongest cell always, which might cause strong interference [50]. On the other hand, in the open access mode all subscribers of a given operator can access the node. This deployment mode reduces the load of the macro cell but might strain the backhaul capacity of the small cells. In hybrid mode, all the subscribers can get access but the quality of service (QoS) is guaranteed only for the subscriber of the CSG [57].

- Relays are operator-installed access points that are typically deployed to cover poor coverage areas and dead zones in the macrocells. The backhaul that connects the relay node to the macro eNB is wireless and uses the air interface resources of the cellular system. Relays transmit the users' data back and forth from and to the macro cell. Therefore, relays are actually an extension of the macro eNB not a fully functional access point. 
- RRHs are low-weight RF units, which are mounted outside the macrocells to extend the coverage of the central eNBs. The RRH has no baseband unit (BBU). RRHs are connected to the Macro eNB (MeNB) or BBU pool via high-speed fiber cable. The central eNBs or BBU pools do all of the signal processing. The BBU pool is composed of BBUs that process baseband signals and optimize the network resource allocation. Therefore, RRHs are deployed for centralized densification instead of distributed densification. RRH can be relatively simple and costeffective.

\subsubsection{Open Issues and Challenges}

Network densification has a significant impact on the improvement of coverage, throughput and spectral efficiency of wireless cellular networks as we stated before. Though, ultra-dense heterogeneous network (UDHetNet) is considered one of the key enablers for $5 \mathrm{G}$ wireless networks, it faces some challenges as well. In this section, we focus on the challenges facing the successful deployment of UDHetNets to achieve the expected performance. Many of the related papers also discuss the challenges of UDHetNets. However, here we summarize the open issues and challenges that require further investigation.

- How much densification can be possible to deploy the eNBs is still an open issue. To define the densification limit we need to consider both access network technologies and backhaul networks. As shown in Table 4, different research shows different values for the number of eNBs per $\mathrm{km}^{2}$. Therefore, cellular densification limit needs to be investigated further. 
Table 4: Number of access points per $\mathrm{km}^{2}$ in UDHetNet

\begin{tabular}{|c|c|c|c|}
\hline \multirow{2}{*}{ Reference } & Traditional & LTE-A with & Next Generation Wireless \\
& Networks & HetNets & Cellular Networks with \\
\hline$[59]$ & $4-5$ eNBs $/ \mathrm{km}^{2}$ & $8-10 \mathrm{eNBs} / \mathrm{km}^{2}$ & $40-50 \mathrm{eNBs} / \mathrm{km}^{2}$ \\
\hline$[65]$ & $7 \mathrm{eNBs} / \mathrm{km}^{2}$ & $21-26$ & \\
\hline$[62]$ & -- & eNBs $/ \mathrm{km}^{2}$ & $93 \mathrm{eNBs} / \mathrm{km}^{2}$ \\
\hline$[52,51]$ & -- & -- & $100 \mathrm{eNBs} / \mathrm{km}^{2}$ \\
\hline$[66]$ & $3-5 \mathrm{eNBs} / \mathrm{km}^{2}$ & -- & $10^{3} \mathrm{eNBs} / \mathrm{km}^{2}$ \\
\hline
\end{tabular}

- Interference management is still one of the most challenging issues for UDHetNets $[57,62,61]$. It is a predominant influence on the operation of a dense HetNets. In [43], the authors also mention that suppressing interference through advanced signal processing techniques to attain the potential gain of UDHetNets is very critical. Therefore, sharing the spectrum needs further study and might need adopting advanced techniques such as CoMP and eICIC (enhanced ICIC) for coordination, exploitation or cancellation of inter-cell interference.

- Several research works and surveys show that most of the operators consider the backhaul as one of the key challenges to small cell deployment $[50,61,67]$. Backhauling is identified as a bottleneck for the widespread deployment of ultradense HetNets. There are some wired, and wireless backhaul solutions proposed 
in the literature in order to address the backhaul needs in dense heterogeneous 5G RAN [61, 60]. In [59], the authors investigated backhaul energy efficiency and capacity of ultra-dense wireless cellular networks and proposed two backhaul distribution architectures. Therefore, the study on wired and wireless backhauling is still an open issue.

- The handover process is used to support the seamless mobility of the UEs in the wireless cellular network. The handover process allows a UE in active mode to transfer from the serving cell to a neighboring cell with the strongest received power without awareness of the user. UDHetNets comprised of different tiers of cells with different frequency bands that intensify the existing challenges of handling handover for UEs. The 3GPP in [9] showed that the increase in the number of handovers in small cell networks compared to macro only networks can be $120 \%-140 \%$ depending on the speed of the user equipment (UE). Moreover, in HetNets a mobile UE cannot consider the same set of handover parameters in all the networks as those used in macro-only networks. Therefore, in UDHetNets, the handover process is also a challenge.

- Energy efficiency plays a significant role in the operating expense of the network, which is an important factor to consider. This is referred as the ratio between the area spectral efficiency and the total power consumed in a network [57]. The maximization of energy efficiency considering the quality of experience $(\mathrm{QoE})$ is an interesting area to be investigated in UDHetNets. To investigate the energy efficiency, we should consider the access networks as well as the backhaul networks. 
Though densification of access points is shown to have a significant impact on the performance of wireless cellular networks, it is important to consider signaling, overhead, computational complexity, cost etc. alongside the above-mentioned challenges measuring the viability of the deployment of UDHetNets. However, as UDHetNets are considered to be a key enabler for the next generation of wireless cellular networks, they should also be studied with another potential enabler that enhances the spectral efficiency such as coordinated multi-point (CoMP) communications. In the following section, we present the state-of-the-art research works in CoMP operation.

\subsection{Multicell Cooperation}

Multicell cooperation such as coordinated multi-point (CoMP) operation was adopted for LTE-Advanced in release 11 to provide coverage of a large number of users with the high data rate, improve the cell-edge throughput as well as the system throughput [27]. Before LTE-Advanced, each cell serves its own users' equipment (UEs). As a result, the UEs in the cell border may receive low signal quality from its serving eNB and high inter-cell interference from the neighboring cells. The serving eNB of a UE is the base station that has the best wireless channel condition to the UE compared to the all other neighboring eNBs. In case of no cooperation this serving eNB usually serves the UE. The core idea of CoMP is to evolve the conventional single-cell multiuser system to multi-cell multiuser systems. In this approach, UEs close to the edge of a cell can be the central point of an area served by multiple eNBs. Therefore, the UEs with low signal quality will get better service by the cooperation of nearby eNBs. For example, in case of the CoMP joint transmission, a UE receives services from more than one eNBs together and the interference changes 
into the useful signal as demonstrated in the following equation [68]. We will discuss different CoMP transmission schemes in subsection 2.3.3 in details.

$$
\begin{array}{clc}
C=B W \log 2\left(1+\frac{p_{S}}{I+p_{N}}\right) & \text { Without CoMP cooperation } & \text { Equation } 2 \\
C=B W \log 2\left(1+\frac{p_{S}+I}{p_{N}}\right) & \text { With CoMP cooperation } & \text { Equation } 3
\end{array}
$$

where, $C$ is the capacity, $B W$ is the bandwidth, $p_{S}$ is signal power, $p_{N}$ is the noise power and $I$ in the interference. In equation 3 , interference converted to a useful signal for a UE. As a result, UE experiences better SINR in CoMP operation that eventually improves the system capacity.

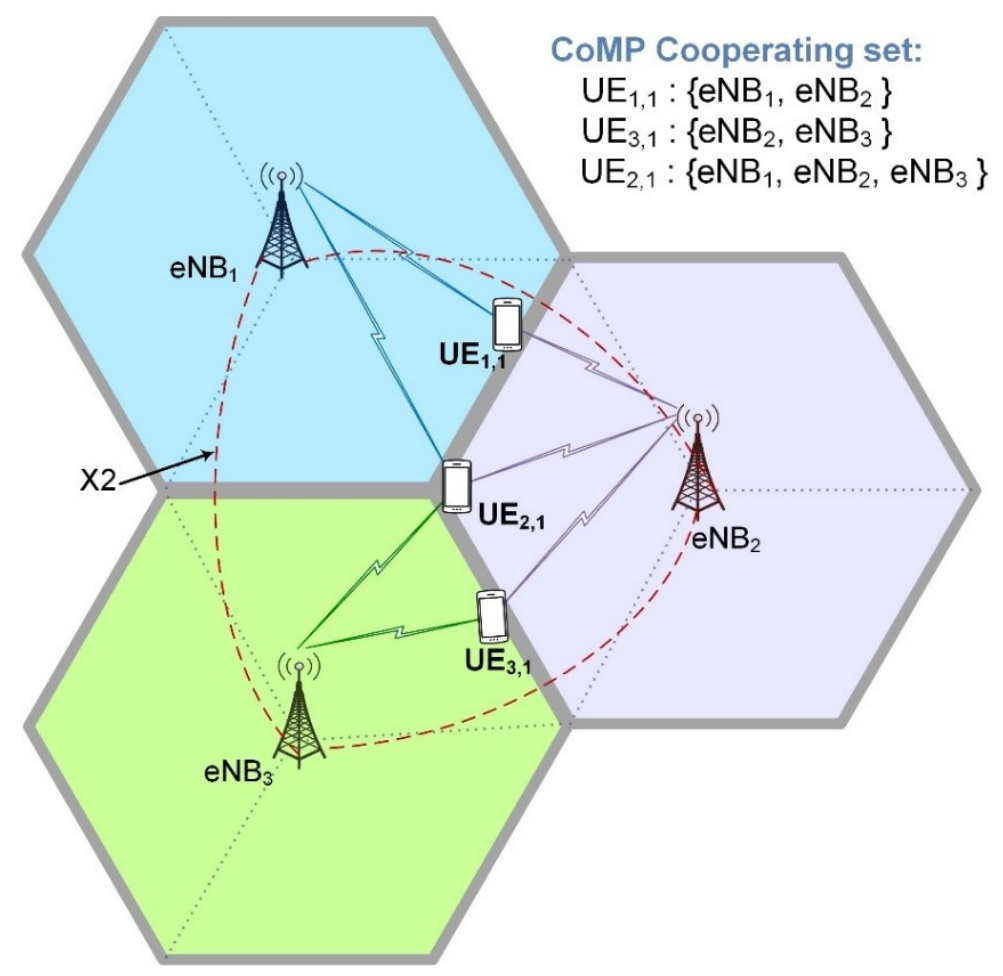

Figure 4: Coordinated multipoint cooperation 
Figure 4 shows the basic diagram of a cooperative communication. As we mentioned before, UDHetNet is a promising technology to achieve the goals of $5 \mathrm{G}$ but inter cell interference (ICI) is extremely serious in UDHetNets due to the dense deployment of small cells and its pseudo-random network topology $[69,8]$. Recent research has shown that CoMP has the potential to improve the performance by mitigating the ICI. Moreover, 3GPP release 14 also included CoMP in the study item for further enhancement focusing on the dense networks [12]. Therefore, although CoMP has been studied in LTE-Advanced as a new ICI management technology, it should be further investigated. We summarize the recent works and key point related to CoMP and UDHetNet in Table 5. 
Table 5: Major related works in CoMP and UDHetNet

\begin{tabular}{|c|c|c|}
\hline Reference & Work area & $\begin{array}{l}\text { Key points presented in the corresponding } \\
\text { referred articles }\end{array}$ \\
\hline $\begin{array}{l}{[27][12]} \\
{[35][70]}\end{array}$ & Technical report & $\begin{array}{l}\text { - } \text { CoMP scenarios } \\
\text { - } \text { Signaling support for CoMP } \\
\text { - } \quad \text { Channel state information (CSI) } \\
\text { - Protocol specification }\end{array}$ \\
\hline $\begin{array}{c}{[71][23]} \\
{[21][26]} \\
{[25][14]} \\
{[68][72]} \\
{[11]}\end{array}$ & Architecture & $\begin{array}{l}\text { - } \text { CoMP Architectures: Centralized, distributed } \\
\text { and user-centric architecture } \\
\text { - } \text { Clustering } \\
\text { - } \text { CoMP overhead } \\
\text { - } \text { CoMP schemes such as JP, CS/CB }\end{array}$ \\
\hline $\begin{array}{c}{[73][74]} \\
{[75]}\end{array}$ & $\begin{array}{l}\text { Interference } \\
\text { coordination }\end{array}$ & $\begin{array}{l}\text { - Interference measurement } \\
\text { - Interference coordination in HetNets } \\
\text { - CoMP for mitigating interference in } \\
\text { heterogeneous cloud small cell environment }\end{array}$ \\
\hline $\begin{array}{c}{[8][49][76]} \\
{[16]}\end{array}$ & $\begin{array}{l}\text { Performance of } \\
\text { CoMP in } \\
\text { UDHetNets }\end{array}$ & $\begin{array}{l}\text { - Performance analysis of CoMP JP, CS/CB and } \\
\text { user-centric in UDN } \\
\text { - Cluster size } \\
\text { - Interference management in UDN }\end{array}$ \\
\hline $\begin{array}{c}{[69][77]} \\
{[78]}\end{array}$ & $\begin{array}{c}\text { Enhancement of } \\
\text { CoMP for } \\
\text { UDHetNets }\end{array}$ & $\begin{array}{l}\text { - } \text { Importance of CoMP for UDN } \\
\text { - } \quad \text { Coordinated spatial resources management } \\
\text { strategies for UDN }\end{array}$ \\
\hline
\end{tabular}




\subsubsection{CoMP Deployment Scenarios}

The 3GPP standardization body considered four different scenarios for the study of CoMP $[72,14,27]$. The first two scenarios focus on homogeneous networks deployment, and the remaining two focus on heterogeneous networks deployment. They are presented in Figure 5.

Scenario 1: Homogeneous networks with intra-site CoMP. A cell site is composed of three sectors (cells), and an eNB controls all the radio resources of the site. In this scenario, external connections between different sites are not required, but the coordination is limited to the sectors of the same site.

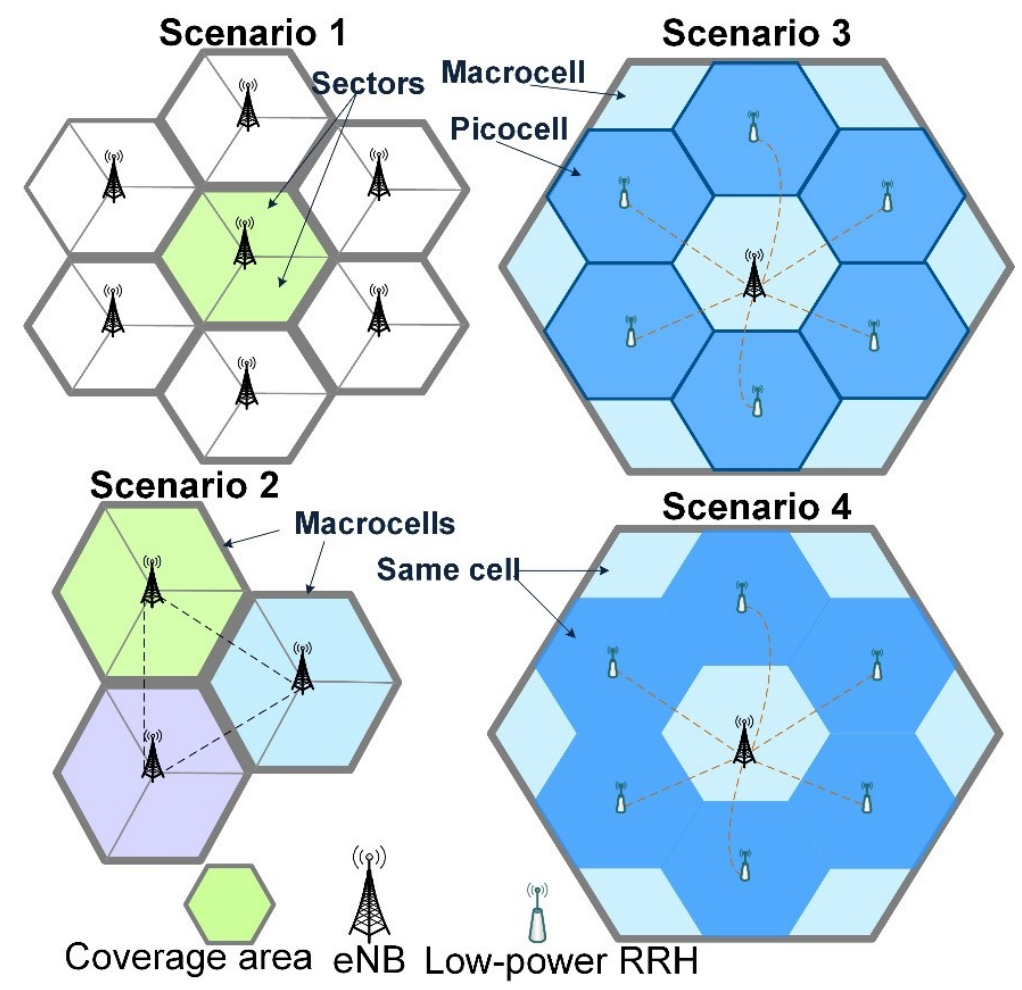

Figure 5: 3GPP CoMP scenarios

Scenario 2: Homogeneous networks with inter-site CoMP. This scenario extends Scenario 1 by including multiple cells of different sites. In this scenario, multiple eNBs at different 
sites coordinate with each other or one controlling eNB and the other high power remote radio heads (RRHs) of different sites within the coordination area. The performance gain of this scenario over Scenario 1 depends on the number of cells involved and the latency of connections between the sites. Scenario 2 in Figure 5 depicts this type of CoMP network with multiple eNBs at different sites [12, 72, 27].

Scenario 3: Heterogeneous network with low-power picocells within macrocell coverage. In this scenario, macrocells with high transmission power and picocells with low transmission power coexist. Each picocell has a low power RRH or Pico eNB connected to the macro eNB within the macrocell coverage area. Each picocell has its own physical cell identity (PCI) independent from the macrocell $[73,79]$. In Figure 5, Scenario 3 depicts one macro eNB and some low power RRH or Pico eNB in each picocell within the macrocell $[72,80,27]$.

Scenario 4: Heterogeneous networks with low power RRHs within the macrocell coverage. The difference between this scenario and the scenario 3 is that all low power RRHs share the same physical cell identity as the macrocell. Since each RRH does not create an independent cell, coordination is done among distributed antennas within a single cell. Consequently, conventional mobility support such as handover procedures among the RRHs is not needed. In addition, low-delay and high-capacity backhaul connection are required between eNB and RRHs [79, 12, 14].

\subsubsection{CoMP Sets}

3GPP specifications define some new terms to distinguish how different cooperating eNBs participate in the coordinated multipoint communication $[68,27]$. The set of cells or eNBs 
that coordinate in order to improve the spectral efficiency is defined as a CoMP set. Following are the three core types of sets used in the CoMP operation as shown in Figure 6.

CoMP cooperating set: The CoMP cooperating set is a set of geographically separated eNBs, directly and/or indirectly participating in data transmission to a UE.

CoMP transmission points: CoMP transmission point(s) are the set of eNBs transmitting data to a UE. CoMP transmission point(s) is (are) a subset of the CoMP cooperating set.

CoMP measurement set: this is a set of eNBs about which channel state information (CSI) is reported by the UE. The CoMP measurement set may be the same as the CoMP cooperating set.

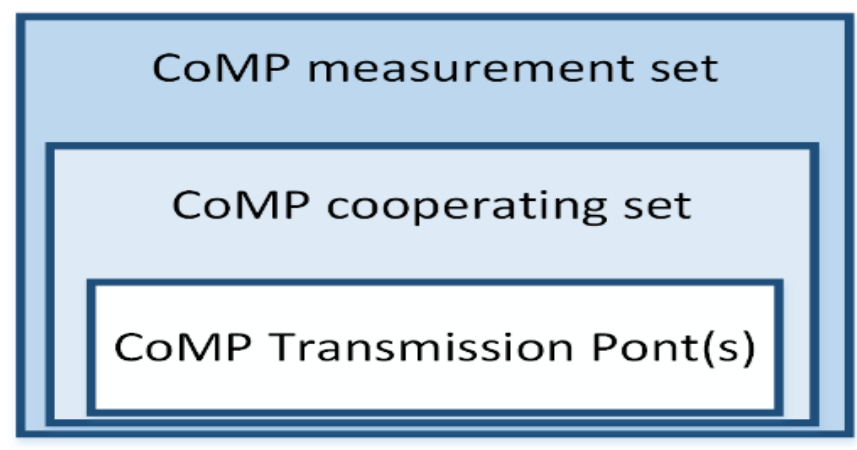

Figure 6: CoMP sets

\subsubsection{CoMP Transmission Schemes}

A variety of CoMP schemes have been identified and proposed. In this section, we outline the downlink and uplink schemes presented in 3 GPP release 11 as well as $14[12,27]$. There are three main types of CoMP transmission schemes: coordinated scheduling/coordinated beamforming (CS/CB), joint processing (JP) and dynamic cell selection (DCS). 


\subsubsection{Joint Processing}

In JP, data for a UE is transmitted jointly from more than one eNBs in the CoMP cooperating set to improve the received signal quality and cancel interference. Cooperating eNBs should exchange both user data and channel information among them. Therefore, low latency and a high bandwidth backhaul are required [12, 72, 27]. Figure 7 shows the CoMP joint processing scheme.

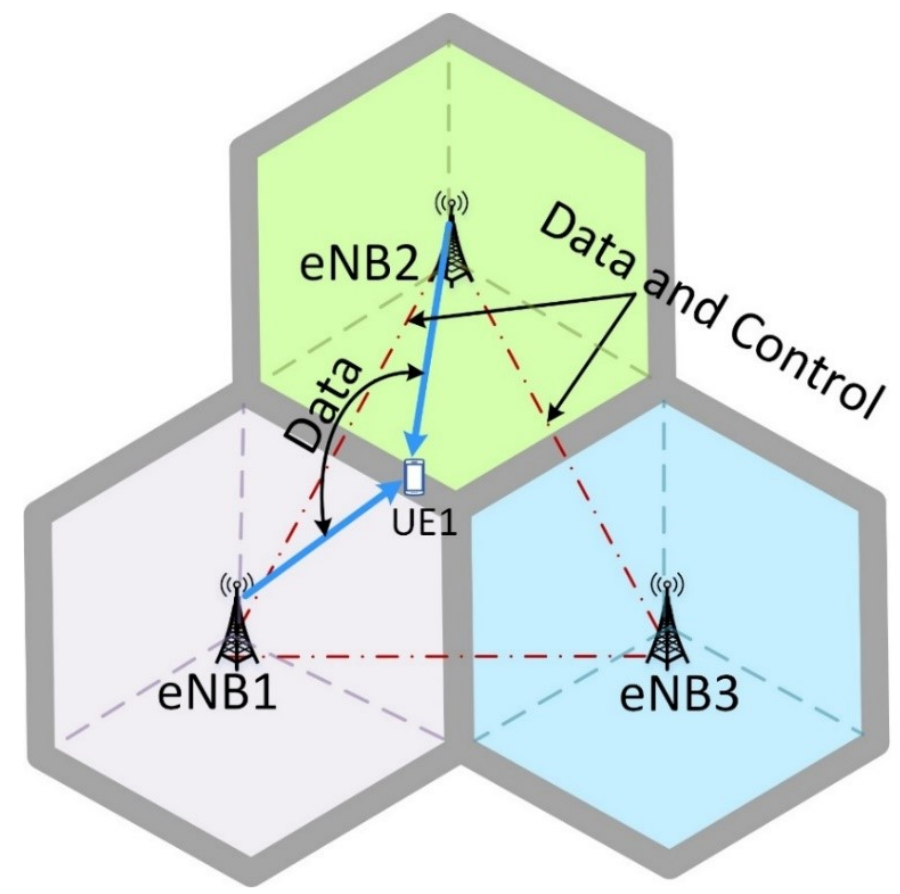

Figure 7: CoMP joint processing (JP)

\subsubsection{Coordinated Scheduling/Coordinated Beamforming (CS/CB)}

In $\mathrm{CS} / \mathrm{CB}$, data for a $\mathrm{UE}$ is only available at one eNB in the CoMP cooperating set but scheduling and/or beamforming decisions are taken with coordination among the eNBs corresponding to the CoMP cooperating set. This coordinated beamforming reduces interference and improves throughput $[12,72,27]$. To perform the scheduling and beamforming eNBs, it is necessary to know the channel status information (CSI). 
Therefore, the UEs need to feedback CSI and it is required to exchange within the cooperating set. In $\mathrm{CS} / \mathrm{CB}$, backhaul load is much lower than JP since only channel information and scheduling decisions need to be exchanged among eNBs [72, 27]. Figure 8 shows the coordinated scheduling/coordinated beamforming $(\mathrm{CS} / \mathrm{CB})$ scheme.

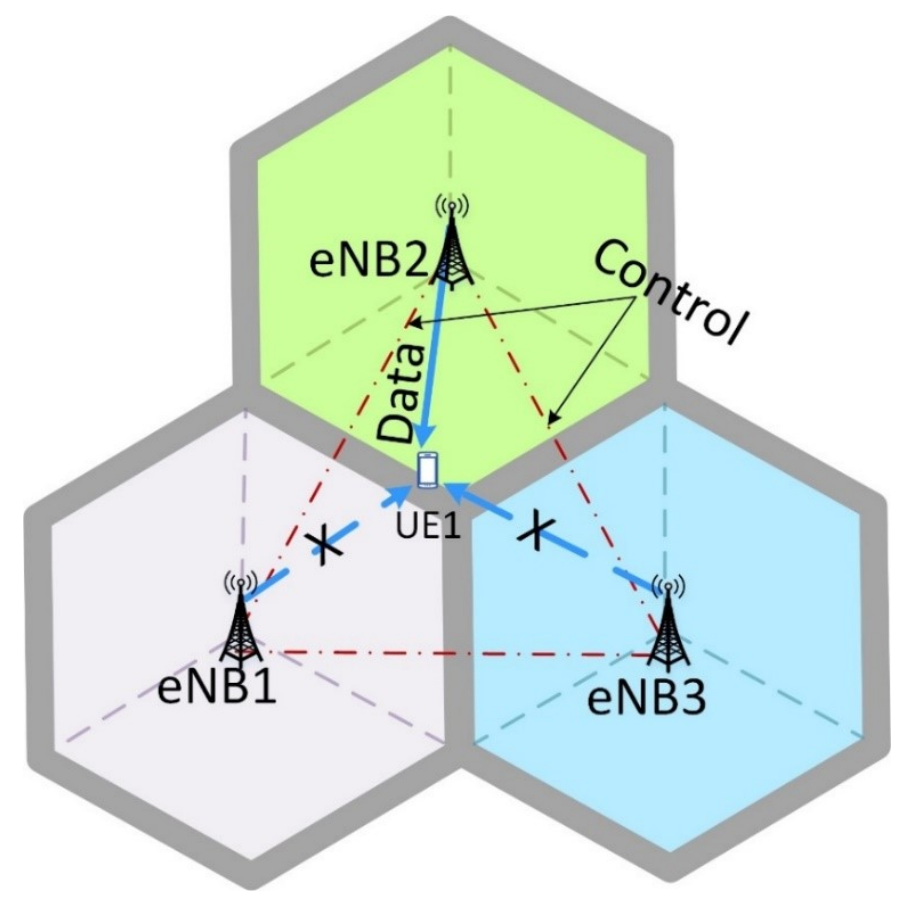

Figure 8: Coordinated scheduling/coordinated beamforming $(\mathrm{CS} / \mathrm{CB})$

\subsubsection{Dynamic Cell Selection}

The UE data is available at multiple eNBs within the cooperating set but at any one time it is transmitted by a single eNB, as shown in Figure 9. This single transmitting/muting point can dynamically change from time-frame to time-frame within the cooperating set to provide the best transmission for a UE [79, 27]. Channel conditions are exploited to select the best serving cell at each sub-frame [11]. 


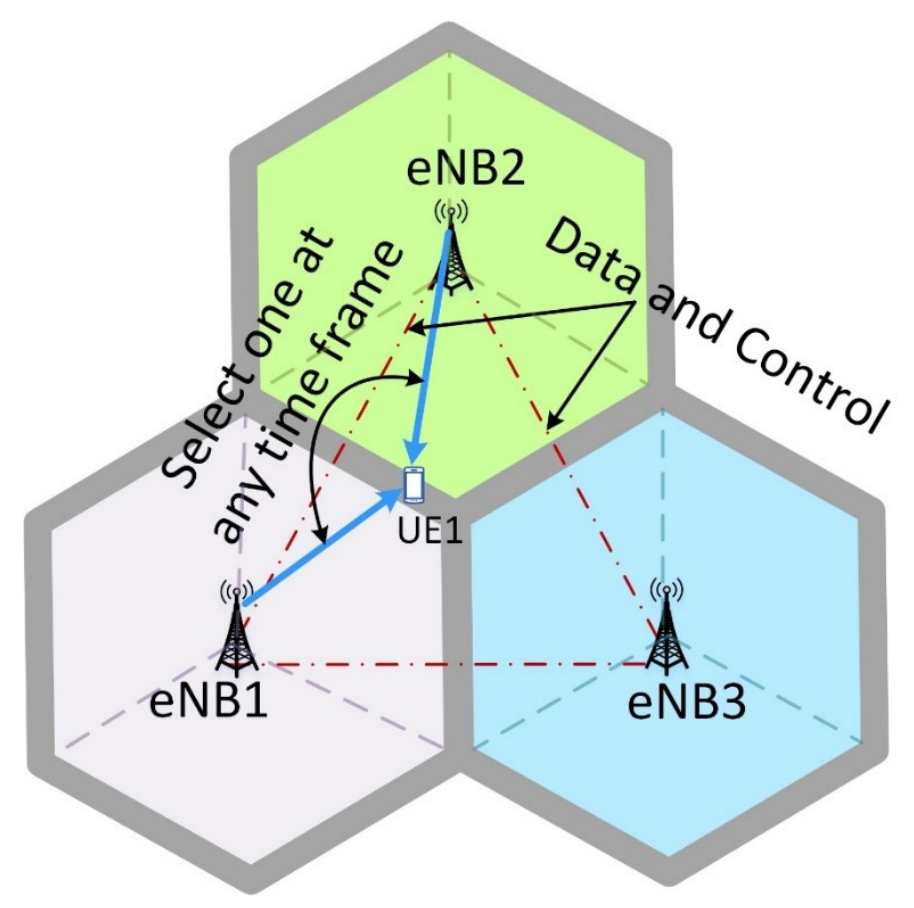

Figure 9: Dynamic cell selection

\subsubsection{Reference Signals and CSI Feedback}

One of the main challenges for CoMP scheme is to obtain accurate channel information with acceptable overhead. In this section we address the issues how required channel knowledge can be obtained and make available for the scheduler. We first discuss about the reference signals those are used for channel estimation in multicell cooperation. After that, we discuss how the obtained channel knowledge or channel state information (CSI) can be efficiently feedback to the transmitter side. Figure 10 shows the basic CSI feedback mechanism in CoMP. 


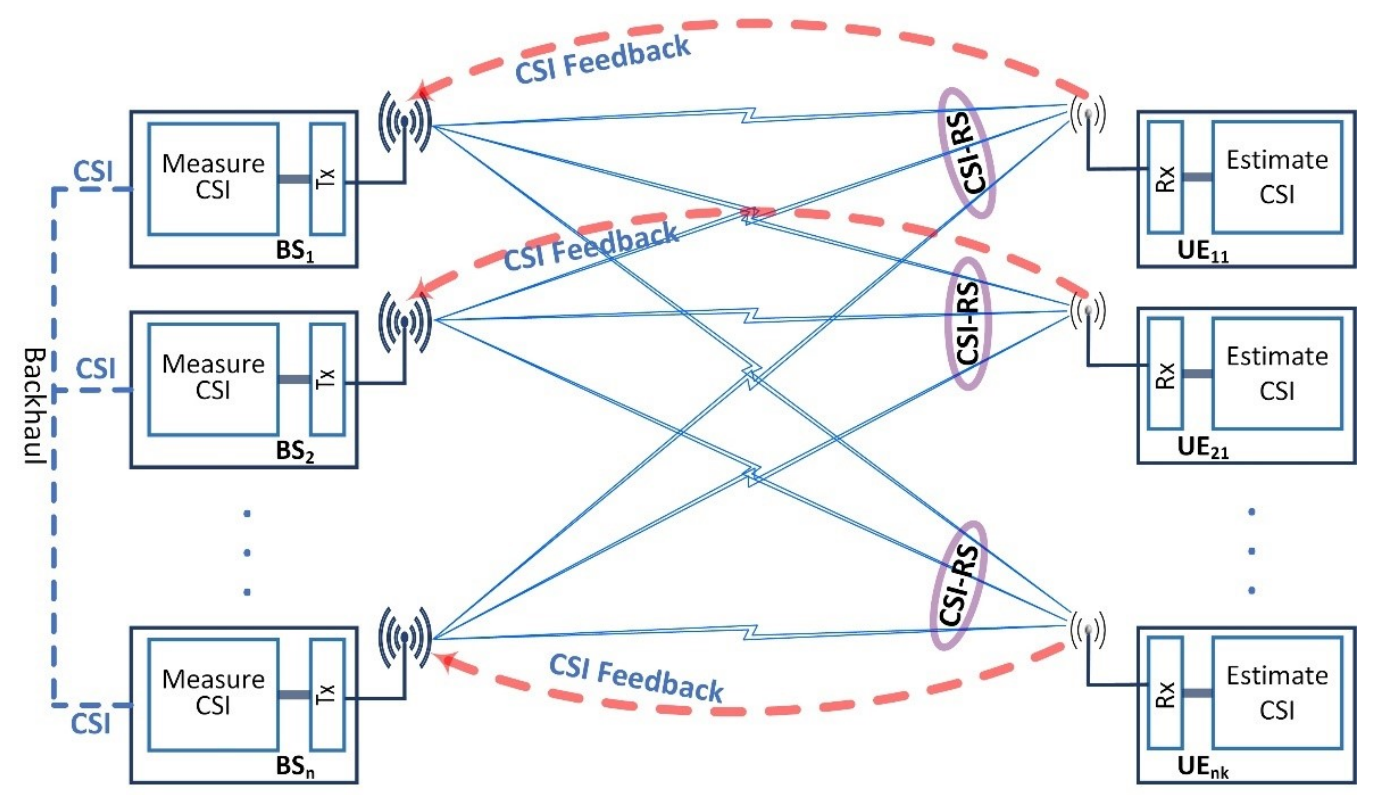

Figure 10: CSI feedback in CoMP

Multi-cell channel estimation is an important issue in cooperative communication, which must be provided by the UEs. Therefore, two new reference signals have been adopted in LTE-A to support CoMP and MIMO. One reference signal is for channel measurement (CSI-RS) and the other one for demodulation (DM-RS) [81, 72]. All the eNBs in the cooperation set provide CSI-RS from which UEs estimate the multicell downlink channel. UEs provide CSI feedback to their serving eNB over the uplink. The cooperating eNBs exchange the received CSI as well as the shared user data over the low latency backhaul denoted as X2 interface.

CSI-RS transmitted from eNB antenna port (AP) to UE in order to estimate the downlink channel quality and determining CSI feedback. It supports a configuration of $1,2,4,8,12$, $16,20,24,28$ or 32 antenna ports and are transmitted on antenna ports $p=15, p=15,16$, $p=15,16,17,18, p=15, \ldots, 22, p=15, \ldots, 26, p=15, \ldots, 30, p=15, \ldots, 34, p=15, \ldots, 38, p=15$ $\ldots, 42$ and $p=15, \ldots, 46$ respectively [81]. A UE uses the CSI-RS for channel estimation. 
Figure 11 shows the CSI-RS mapping patterns for 8 port system in physical resource blocks (PRB) $[68,82,83]$. In Appendix B, we discussed the transmission scheme and physical resources of wireless networks. The CSI-RS patterns have large reuse factor depending on the number of antenna ports. In case of 1, 2, 4 and 8 antenna ports, CSI-RS has 20, 20, 10 and 5 reuse factors respectively $[68,83]$. The CSI-RS reuse patterns allow different eNBs to avoid a mutual CSI-RS collision. The density of CSI-RS effects on the channel estimation accuracy. In general, higher CSI-RS density provides better CSI estimation accuracy while reducing downlink resource utilization. Therefore, to reduce the CSI-RS overhead, the transmission frequency is considered every $5,10,20,40$ or $80 \mathrm{~ms}[83,72$, 84]. Now, let consider transmission frequency is $10 \mathrm{~ms}$. In this case, 1 symbol will be transmitted in every $10 \mathrm{~ms}$ per antenna port, which is 1 symbol in 140 transmitted symbols in the time domain. In the frequency domain, 1 subcarrier in every 6 subcarriers per antenna port which is 1 symbol in 6 transmitted symbols. As a result, CSI-RS overhead in the downlink is $1 / 840=0.12 \%$ per antenna port. For 8 antenna port it will be $0.96 \%$ and for 30 antenna port it will be $3.6 \%$ [68]. 


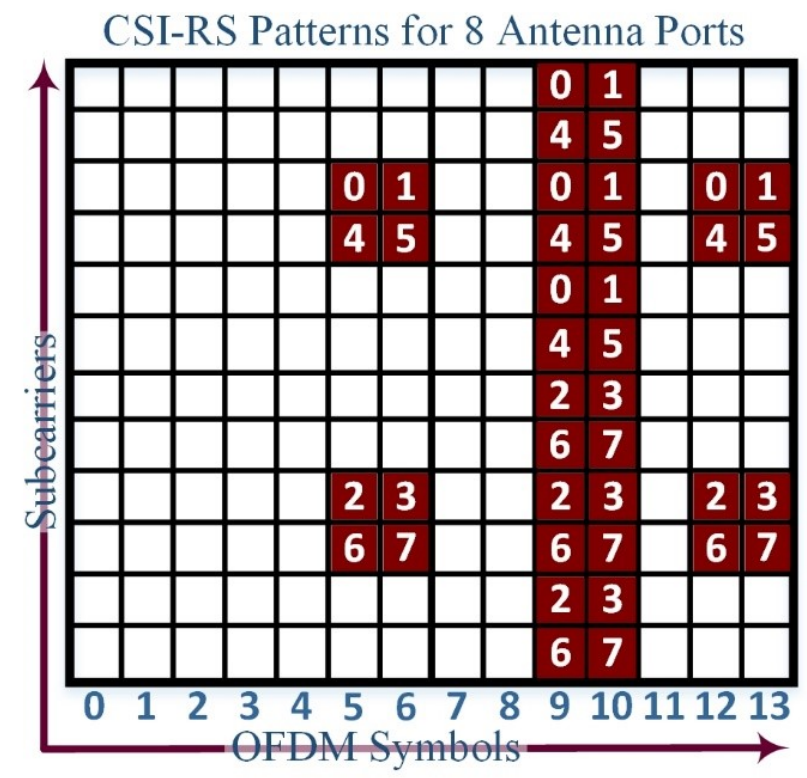

Figure 11: CSI-RS pattern for 8 antenna ports

Regarding the download CoMP transmission, the network needs information related to the downlink channel condition, so that eNBs can perform the appropriate radio resource management and adaptive transmission. Therefore, a UE needs to estimate the channel state information (CSI) of all the neighboring cells using received CSI-RS and report it to the serving eNB. The CSI and scheduling information need to be exchanged among all the cooperating eNBs over the limited backhaul interface. The throughput of a downlink CoMP channel heavily relies on the quality of the CSI feedback available at the transmitter [21, $14,73]$. In the next subsection we discuss different types of CSI feedback mechanism.

\subsubsection{Types of CSI feedback Mechanism}

There are two main categories of CoMP feedback mechanisms: explicit CSI feedback and implicit CSI feedback. 


\subsubsection{Explicit CSI feedback}

This type of feedback mainly includes direct channel coefficient, spatial channel covariance matrix and principle eigenvector. For example, the feedback transmission for the $\mathrm{k}_{\mathrm{th}} \mathrm{UE}$ can be stated as:

$$
y_{C S I-R S_{k}}=H_{k} W_{C S I-R S} x_{C S I-R S}+n_{k}, \quad \text { Equation } 4
$$

where, $H_{k} i$ is the channel matrix connected to UE k. $W$ is the precoding matrix and $x$ are the CSI reference symbols. $n_{k}$ is the additive Gaussian noise at receiver $\mathrm{k}$.

This is also considered as accurate channel information feedback or full CSI feedback. Therefore, this feedback scheme can deliver the best performance, but feedback overhead is very high. For example, in a 2 cell CoMP cooperation scenario with $20 \mathrm{MHz}$ bandwidth and $10 \mathrm{~ms}$ feedback periodicity, the CSI feedback overhead is in terms of several Mbp/s, which is unacceptable for the commercial system $[18,85]$. Therefore, feedback reduction technique is very important to achieve the goal of CoMP cooperation.

\subsubsection{Implicit CSI feedback}

In this approach a UE makes transmission recommendation based on the precoding codebook, which is known at the eNB and the UE. It can not accurately describe the channel matrix as explicit feedback but has lower feedback overhead. The implicit CSI feedback reflects the recommended rank indicator (RI), a precoding matrix indicator (PMI), and channel quality indicator (CQI). The RI is the preferred transmission rank of a number of usable data streams or layers available for CoMP transmission. The precoding matrix determines how the individual data streams are mapped to the antennas. The 
received PMI indicates which precoding matrix should be employed for downlink transmission to an eNB. The CQI reflects the channel quality corresponding to the reported PMI $[73,14]$.

According to the 3GPP specifications and other literature, four widely recommended codebook based compressed CSI feedback schemes are Wideband, Subband, Best-M and Full feedback $[86,87]$.

- Wideband: Each UE transmits one single 4-bit CQI value describing the channel quality for all of the PRBs in the bandwidth in every reporting period. In the wideband scheme the CQI feedback overhead is given in equation 5.

$$
O_{f b_{C Q I-W B}=2 .\left(4 . N_{U E}\right)}, \quad \text { Equation } 5
$$

where, $N_{U E}$ is the number of UEs served in the CoMP operation.

- Subband level: The bandwidth is divided into $N_{\text {sband }}$ subbands of $N_{R B}^{\text {sband }}$ consecutive resource blocks. Each user feeds back to the base station one 4 bits wideband CQI and 2 bits differential CQI for each subband. The number of consecutive resource blocks in a subband $N_{R B}^{s b a n d}$ is dependant on bandwidth as shown in Table 6 [86] and the CQI overhead model is shown in equation 6.

$$
O_{f b_{C Q I-S B}=2 \cdot\left(4+2 \cdot N_{\text {Sband }}\right) \cdot N_{U E} \quad \text { Equation } 6}
$$


Table 6: Subband size according to system bandwidth for Subband level feedback

\begin{tabular}{|c|c|}
\hline System Bandwidth $\left(\boldsymbol{N}_{\boldsymbol{R}}^{S B}\right)$ & Number of RBs in a Subband $\left(N_{R B}^{\text {sband }}\right)$ \\
\hline $6-7$ & NA \\
\hline $8-10$ & 4 \\
\hline $11-26$ & 4 \\
\hline $27-63$ & 6 \\
\hline $64-110$ & 8 \\
\hline
\end{tabular}

- UE selected Best-M: Each UE selects M preferred subbands of equal size $N_{R B}^{\text {sband }}$ as shown in Table 7 [86]. UE feeds back to the eNB one 4 bits wideband CQI and 2 bits differential CQI that reflects the channel quality only over all the selected $\mathrm{M}$ subbands. In this scheme, UE also report the position of these subbands in the bandwidth. Equation 7 shows the CQI feedback overhead for this feedback scheme.

$$
O_{f b_{C Q I-B M}}=2 \cdot\left(4+2+\left[\log _{2}\left(\begin{array}{c}
N_{R B}^{S B} \\
M
\end{array}\right)\right]\right) \cdot N_{U E} \quad \text { Equation } 7
$$


Table 7: Subband size and corresponding selected number of subbands according to the system bandwidth

\begin{tabular}{|c|c|c|}
\hline $\begin{array}{c}\text { System Bandwidth } \\
\left(\boldsymbol{N}_{\boldsymbol{R B}}^{S B}\right)\end{array}$ & $\begin{array}{c}\text { Number of RBs in a } \\
\text { Subband }\left(N_{R B}^{\text {sband }}\right)\end{array}$ & $\begin{array}{c}\text { Selected number of } \\
\text { Subbands (M) }\end{array}$ \\
\hline $6-7$ & NA & NA \\
\hline $8-10$ & 4 & 1 \\
\hline $11-26$ & 4 & 3 \\
\hline $27-63$ & 6 & 5 \\
\hline $64-110$ & 8 & 6 \\
\hline
\end{tabular}

- Full Feedback: In this scheme, each UE reports a 4-bit wideband CQI value and a 2-bit differential CQI for each RB.

$$
O_{f b_{C Q I-F}}=2 \cdot\left(4+2 \cdot N_{R B}^{S B}\right) \cdot N_{U E} \quad \text { Equation } 8
$$

In CoMP operation, cooperative eNBs also require exchanging received CSI among them, resulting in additional CSI delay. The performance of CoMP transmission and reception is also sensitive to the delay of CSI exchange. This CSI delay and the signaling overheads are mainly influenced by two factors, CoMP coordination architecture and backhaul technology. In the next subsection, we discuss the coordination architectures of CoMP operation. 


\subsubsection{CoMP Coordination Architecture}

The coordination architecture of CoMP can be defined as the way participating cell sites coordinate to exchange information, handle interference and scheduling. There are two kinds of coordination architecture can be categories for CoMP transmission and reception with respect to the way this information is made available at the different transmission point: centralized and distributed. However, the existing CoMP architectures suffer some overhead related to signaling and infrastructure of the network that will be discussed later $[25,22,88,19,15]$

\subsubsection{Centralized Architecture}

In the Centralized architecture, a central unit $(\mathrm{CU})$ is responsible for handling radio resource scheduling by centrally processing the feedback information from the cell sites. At first, the UEs estimate the CSI related to all the cooperating eNBs and feed it back to their serving eNB, which forwards the local CSI to the CU. Finally, the CU calculates the precoding information for all of the eNBs in the cooperation set, and based on that, it takes the scheduling decisions and communicates them to the cooperating eNBs, as shown in Figure 12. This framework suffers from backhaul signaling overhead and infrastructure overhead as well as increase the network latency [71, 23, 19]. 


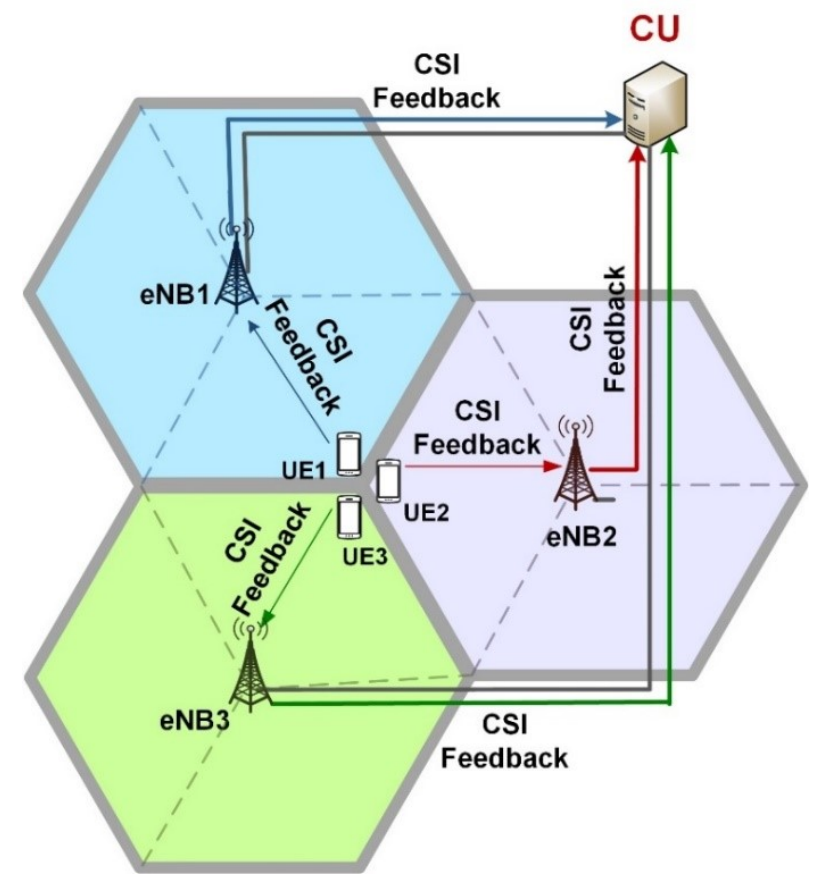

Figure 12: Centralized Architecture

\subsubsection{Distributed Architecture}

In a distributed architecture, the coordinated cells exchange data and channel state information (CSI) over a fully meshed signaling network using an X2 Interface. Prior to downloading, the UEs estimate the CSI related to all the cooperating eNBs and feeds it back to the serving eNB. All the eNBs in the cooperation set share the received CSI and locally compute the precoding information using the same scheme. The eNBs are scheduled independently based on their acquired CSI. This architecture increases the feedback transmission in the backhaul and is sensitive to cooperation set. Moreover, in this case UE data might also need to share through $\mathrm{X} 2$ interface based on the CoMP transmission scheme. This could potentially cause a further performance degradation [71, 23]. Figure 13 shows the CoMP distributed architecture. 


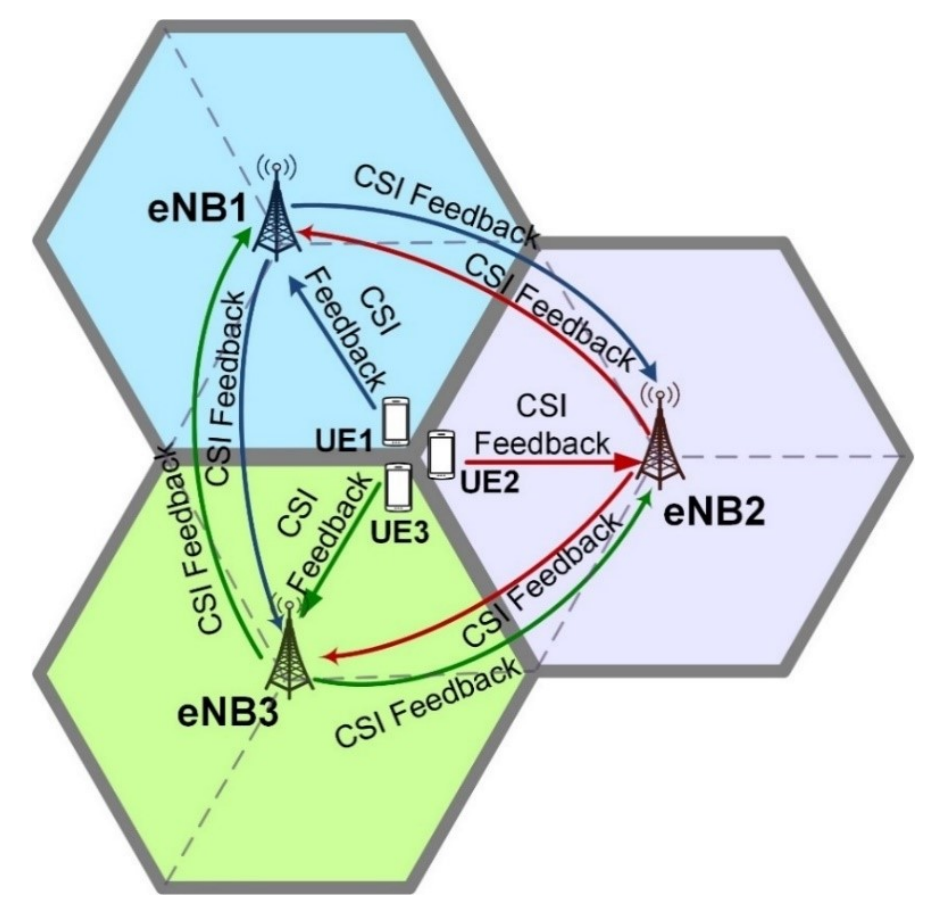

Figure 13: Distributed Architecture

\subsubsection{Backhaul Issues}

With the rise of new enabling technologies for $5 \mathrm{G}$ networks as we stated before, the backhaul network has evolved to a more complex composed of fronthaul, midhaul, and backhaul. The backhaul section connecting the remote radio head (RRH) to the baseband unit (BBU) or the eNB directly is labeled fronthaul. The inter-eNB, and eNBs and small cells link based on X2 interface are called the midhaul. While the network connections between eNBs and the core such as MME and SGW, based on the S1-interface have retained the term backhaul. In this research we use the term backhaul including fronthaul, midhaul, and backhaul. To achieve the promised gain of multicell cooperative networks, two key requirements are the high backhaul bandwidth and the low latency $[11,89,90]$. 
Multicell cooperation requires backhaul to exchange the following information in both uplink and downlink.

- Channel state information (CSI)

- Scheduling information

- Signaling information and

- User data based on CoMP scheme

Inter eNB communication or backhaul communication is not as sensitive in the non-CoMP system as in the CoMP enabled system in the context of both capacity and latency. Depending on the type of CoMP, backhaul requirement will differ. CoMP joint transmission (JT) required more bandwidth than CoMP coordinated scheduling and beamforming $(\mathrm{CS} / \mathrm{CB})$ because of user data being shared among the cooperating eNBs. For example, according to the literature, CoMP CS/CB requires backhauling on the order of several hundred kbps. On the other hand, CoMP JT requires backhauling up to several hundred Mbps [90, 84]. CoMP backhaul capacity and latency requirements are investigated in detail in $[84,85]$. In $[91,92]$, the authors analyzed the feasibility of CoMP cluster size in different backhaul network technologies and topologies. They also presented, how backhaul network link capacity and the delay limit the feasibility of wireless cooperation. In [90, 11], authors studied detail about the backhaul requirements for CoMP transmission and reception. Traditional link technologies merely provide sufficient bandwidth just for the user data possible with LTE and LTE-A, not sufficient for additional backhaul bandwidth that is required for CoMP operation in the future networks [84]. Therefore, CoMP enabled radio access networks require new backhaul link technologies that improve the capacity and reduce the backhaul signaling overhead. To this end, different potential 
technologies presented in the different recent literature. Micro-wave 6-42 GHz, Millimeterwave $60 \mathrm{GHz}$ and 70-80 GHz (E-band) could be attractive for high capacity short links [93, 61]. Fiber point to point, $10 \mathrm{G}$ ethernet passive optical networking (10G-EPON), optical transport network (OTN) with wavelength division multiplexing (WDM) are some potential wired technologies to significantly increase the backhaul capacity [94, 61]. However, for future generation backhauling, wireless solutions have been attracting interest due to their implementation flexibility and cost as well as the architecture of the networks. Addressing this, 3GPP also specifies X2/Xn interface for the backhaul link among the eNBs in LTE-Advanced and beyond networks. The X2 is a logical point-topoint interface between two eNBs within the evolved terrestrial radio access network (EUTRAN). This logical point-to-point interface is possible even though there is no direct physical connection between the two eNBs. In addition to exchanging the above-mentioned information, the $\mathrm{X} 2$ interface supports mobility, radio resource management and dual connectivity between the eNBs and UEs [95]. The objective of X2 interface specification is to facilitate inter-connection among the eNBs supplied by different manufacturers, to support continuation between eNBs of the E-UTRAN services offered via the S1 interface, and to provide separation of $\mathrm{X} 2$ interface radio network (RN) functionality and transport network (TN) functionality for introducing future technology. Two different protocol stacks are defined, one for the user plane (X2-U) and one for the control plane (X2-C). 

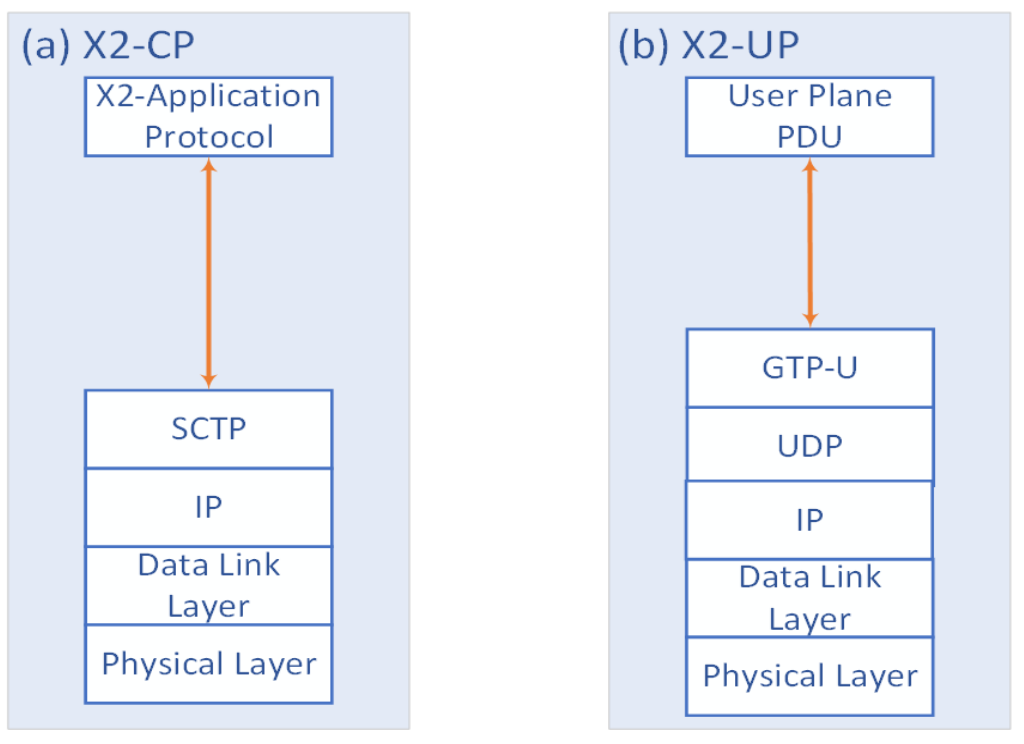

Figure 14: X2 protocol stack

The user plane protocol stack on the X2 interface is shown in Figure 14(b). The X2-U interface provides non-guaranteed user data transfer. The transport network layer uses GPRS tunnel protocol (GTP) user plane (GTP-U) on top of user datagram protocol (UDP) to carry the user plane PDUs. The control plane protocol stack on the X2 interface is shown in Figure 14(a). Stream control transmission protocol (SCTP) is used in this stack to provide reliability and flow control [82]. The application layer signaling protocol is referred to as X2 application protocol (X2-AP). The X2 control plane support handover coordination, dual connectivity, load management, interference coordination, radio resource management and general X2 management.

However, because of the backhaul latency, the CSI exchange may be delayed among the cooperating eNBs to $10 \mathrm{~ms}$ or more [96]. 3GPP in [27] presented how the feedback latency impact the performance of CoMP networks as shown in Table 8. 
Table 8: Performance evaluation of different CoMP schemes with varying CSI feedback delay

\begin{tabular}{|c|c|c|c|}
\hline \multirow{2}{*}{$\begin{array}{c}\text { Transmission } \\
\text { Scheme }\end{array}$} & $\begin{array}{c}\text { Feedlback Delay } \\
\text { (UE to eNB+ Backhaul) }\end{array}$ & $\begin{array}{c}\text { Cell Edge UE (5\%-ile) } \\
\text { Throughput (kbps) }\end{array}$ & Gain (\%) \\
\hline \multirow{3}{*}{ CS/CB } & $5 \mathrm{~ms}+0 \mathrm{~ms}$ & 538 & - \\
\cline { 2 - 4 } & $5 \mathrm{~ms}+5 \mathrm{~ms}$ & 516 & $-4.1 \%$ \\
\cline { 2 - 4 } & $5 \mathrm{~ms}+10 \mathrm{~ms}$ & 477 & $-11.3 \%$ \\
\cline { 2 - 4 } & $5 \mathrm{~ms}+15 \mathrm{~ms}$ & 443 & $-17.7 \%$ \\
\hline \multirow{3}{*}{ JT } & $5 \mathrm{~ms}+0 \mathrm{~ms}$ & 828 & - \\
\cline { 2 - 4 } & $5 \mathrm{~ms}+5 \mathrm{~ms}$ & 776 & $-15.6 \%$ \\
\cline { 2 - 4 } & $5 \mathrm{~ms}+10 \mathrm{~ms}$ & 699 & $-26.6 \%$ \\
\cline { 2 - 4 } & $5 \mathrm{~ms}+15 \mathrm{~ms}$ & 608 & \\
\hline
\end{tabular}

Hence, the success of cooperative communication also depends on the design, latency, and bandwidth of the backhaul since a large amount of control and user data may need to be exchanged among the eNBs [95]. For further improvement, in this year, 3GPP outlined a new draft of Xn interface considering the next generation networks. The objective of Xn interface is to provide extended functionalities of $\mathrm{X} 2$ interface in the context of next generation radio access network (NG-RAN) architecture as shown in Figure 1 [97]. 


\subsubsection{Challenges of CoMP}

There is no doubt that CoMP will continue attracting the attention of researchers and industry, as the next generation networks techniques such as UDHetNet and MIMO need to improve spectral efficiency by coordinating interference. Therefore, some of the significant issues that need to be reinvestigated with respect to the next generation ultradense heterogeneous networks are outlined as follows.

- CoMP performance relies heavily on the efficiency of the CSI in the network. In dense deployment networks, it is difficult providing CSI to all the coordinated eNBs. Moreover, the CSI feedback might consume scarce control resources, which could overwhelm the network. However, exploiting the CSI is necessary for cooperative communication in UDHetNets. Thus, CSI feedback needs further investigation in the context of the next generation cellular networks $[73,72,98]$.

- The benefits of CoMP greatly depend on the coordination among the eNBs, which requires the high capacity of backhaul links. In practice, the capacity of backhaul links is restricted by the deployment scenarios and cost. In the next generation of ultra-dense HetNets, the backhaul problem will become even more serious because of the density, heterogeneity and the randomness of the cells. Therefore, backhauling technologies in CoMP demand more investigation with respect to UDHetNets [72, 16, 49].

Having addressed the above issues, some other issues might also need to reinvestigate such as reference signals (RS) design and mobility management for CoMP operation in the UDHetNets. In this research work we focus on CSI feedback coordination to reduce the 
signaling overhead and feedback delay, and mobility management in the context of dense heterogeneous networks as presented in Chapters 3 and 4. Simulation model is used for analyzing the behavior and performance of a network in scientific research. In the next section we present a brief overview on verification and validation process of simulation model.

\subsection{Verification and Validation of Simulation Model}

Computer modeling and simulation $(M \& S)$ of systems have been used in scientific research for analysis, design and prediction of behavior and performance under different scenarios, settings and environments. Nowadays, the impact of $M \& S$ is immense in the scientific research and decision making. Consequently, the credibility of the computational results is of great concern to researchers, engineers, policymakers, and those who are affected by the decisions based on this analysis and prediction.

The primary process for ensuring the credibility and accuracy of the computational results of simulation model is known as verification and validation $(\mathrm{V} \& \mathrm{~V})$. $\mathrm{V} \& \mathrm{~V}$ is concerned with having a correct model and simulation results for the specific experiments. Verification deals with building the model correctly. On the other hand, validation is concerned with developing a correct model. Model validation is the process of determining the degree to which a model is an accurate representation of the real system from the objective of the study. A model is only validated with respect to specific experiment. It cannot be considered that a model is valid for one experiment is also valid for another experiment. Therefore, the philosophy of verification and validation is basically based on the concept of ensuring the accuracy to build the confidence on the results of the analysis. 
In this section a historical review of the development of $\mathrm{V} \& \mathrm{~V}$ and related works that have been done in the same area are presented very briefly.

Last few decades, many efforts have been devoted to verification and validation process to improve the credibility of the simulation model. Some of the key contributors in the field of M\&S are Naylor and Finger [99], Banks [100], Balci [101, 102], Shannon [103, 104], Zeigler [29], Sargent [105], Neelamkavil [106], Oberkampf [107] etc. A very generic definition of a model for a system is given by Neelamkavil [106].

A model is a simplified representation of a system or process intended to enhance our ability to understand, predict and possibly control the behavior of the system.

Bernard P. Zeigler in his book [29] defined the model in the systems specification formalisms perspective. He mentioned that the most common concept of a model is:

A model is a set of instructions, rules, and equations for generating input/output behavior of a system or a component of a system.

According to the system engineering viewpoint, a system is a set of physical or artificial entities or processes that interact and accomplish some purpose. It could be a proposed or an existing system. According to the systems specification formalisms, a model also describes state transitions and output generation mechanisms to accept input trajectories and generate output trajectories depending on its initial state knowledge. For the more complex system the complete model is constructed by coupling the sub-models or atomic models together. 
To run a model generating its behavior a computation system is needed. There are also several variants of the definition of simulator or simulation in the literature; here we try to present a generic definition.

A simulator is a set of instructions that are capable of executing a model to generate its expected behavior or produce the results.

Computer simulation enabled engineers and scientists to experiment and analyze the behavior of existing or proposed systems within a virtual environment. Simulation model also can be used for training purpose because of its many advantages such as cost, flexibility, risk, over the physical experiment.

As we mentioned above, the ultimate objective of verification and validation is to improve the credibility of the results of analysis. Verification is concerned with building the model correctly. That is verification is the attempt to ensure that the simulator carries out the model instructions correctly. In other words, verification process focuses on the identification and removal of errors in the software implementation (computerized model or simulation model) of the system's model. On the other hand, in validation, the relationship between the model and the real system is the issue. There are several verification and validation processes or techniques are also available in the literature [108, 105, 29]. Accordingly, model verification and validation process could have both conceptual or informal and formal components. There are many definitions of verification and validation available in the literature. Verification and validation are defined in a number of ways in different communities such as the society for computer simulation (SCS) defines V\&V in [109], which is a key milestone. 
Model verification: substantiation that a computerized model represents a conceptual model with specified limits of accuracy.

Model validation: substantiation that a computerized model within its domain of applicability possesses a satisfactory range of accuracy consistent with the intended application of the model.

In these definitions two important terms are conceptual model and computerized model. Conceptual model means a verbal description, equations, governing relationships, or natural laws that significance to describe the system of interest. This model is developed by observing and analyzing the system or process of interest. Computerized model defines as an operational computer program which implements a conceptual model. According to the SCS definition, model verification implies that computer program (simulation model) must accurately mimic the conceptual model. Thus, verification deals between conceptual model and simulation model. The SCS definition of validation focuses on the accuracy of the model. Hence, validation deals between the conceptual model and the reality or the system.

Gradually computer-controlled systems become essential and widespread in the industry and the public system. The institute of electrical and electronics engineers (IEEE) defined verification and validation. IEEE has a strong influence on different organization worldwide and the prevalence of electrical and electrical engineers.

Verification: The process of evaluating the product of a software development phase to provide assurance that they satisfy the conditions imposed by the previous phase. 
Validation: The process of evaluating a system or model during or at the end of the development process to determine whether it satisfies specific requirement.

The definitions provide a scientific or engineering perspective towards the entire issue of verification and validation. In the contemporary period, the US Department of Defense (DoD) also recognizes the importance of different terminology of M\&S. As a result, DoD also defined the verification and validation process [110].

Verification: The process of determining that a model or simulation implementation and its associated data accurately represent the developer's conceptual description and specifications.

Validation: The process of determining the degree to which a model or simulation and its associated data are an accurate representation of the real world from the perspective of the intended uses of the model.

The key feature of the DoD definition is that it emphasizes on accuracy. The DoD definition is more likely to the SCS definition than the IEEE definition. Though, there are many other definitions and methodology on $\mathrm{V} \& \mathrm{~V}$ as we mentioned earlier, we presented the above three definitions because these three organizations have worldwide influence on scientists, engineers and industries.

Accordingly, we can say that validation is the process to establish the validity of the structure of the model and the accuracy of the behavior of the model to reproduce the behavior of the system of interest for the experiment(s). On the other hand, verification is 
the process to ensure the accuracy of model transformation and establishing the correctness of the simulator.

The proposed common framework that accommodates both formal and informal techniques of the verification and validation process and where they fit into the modeling and simulation lifecycle will be discussed in Chapter 5. In the next chapter we discuss the DCEC CoMP coordination architecture in details. 


\section{Chapter: Coordinated Multi-Point Communication Using}

\section{Dynamic Coordination Station}

Multi-cell multi-tier cooperative communication is a key enabler to realize the goals of next generation wireless cellular networks. The idea of coordinated multipoint (CoMP) communication is to evolve from the conventional single-cell multi-user system to a multicell multi-user system. In the cooperative communication, the UEs close to the cell edge can be considered in the central point of the coverage area served by multiple eNBs. In CoMP-enabled systems, the eNBs are grouped into a cooperating set. The eNBs of each of these cooperating sets exchange information among them, and they process signals and provide services to the users jointly. As a result, the UEs can receive their signals simultaneously from single or multiple transmission points in a coordinated or jointprocessing method, which can improve data rate coverage and cell edge throughput [14, $15]$.

However, in CoMP enabled networks, the scheduler needs accurate and updated channel state information (CSI) for adaptive transmission, as well as appropriate radio resource management (RRM) [14, 17]. In order to provide this information to the scheduler, the UEs estimate the CSI and report it to their serving eNB periodically. The coordinated eNBs exchange the received CSI and/or data among them providing services to the UE. This results in a significant increase of signaling overhead and feedback latency into the 
cooperative networks. These overhead and latency are the key challenges to achieve the expected gains in coordinated multi-point (CoMP) operation [14, 96, 27].

The overhead depends on the CoMP coordination architecture. According to the literature, there are two types of coordination architectures available: centralized and distributed [21, 22, 23] as we discussed in Chapter 2. In the centralized architecture, a central unit is responsible for handling radio resource scheduling by processing the CSI feedback information received from the UEs. This architecture suffers from signaling overhead and infrastructure overhead. It also increases the latency of the CSI feedback. In the distributed architecture, the coordinated cells exchange CSI over a fully meshed signaling network using X2 interfaces. This architecture also increases the signaling overhead into the network and highly sensitive with the size of the cooperation set. These signaling overhead and latency are the key causes for performance degradation of cooperative cellular networks $[22,11]$.

In this chapter we present a user-centric dynamic CoMP coordination architecture named Direct CSI-feedback to Elected Coordination-station (DCEC) for minimizing the signaling overhead and feedback latency $[24,25,26]$. In this architecture one of the cooperating eNBs will be dynamically elected as a coordination station (CS) for a UE in the cell edge area. Thereon, the CS will analyze the received CSI information, will determine the cooperating set, and will be in charge of scheduling. Each of the UEs in the cell edge area will go through the same process and UEs in the same CoMP cooperating set will send the CSI feedback to the CS only, which will reduce the signaling overhead into the network. Moreover, no additional hardware is needed for this solution, so the costs for switching to such architecture should be small. 
The whole idea of the DCEC coordination architecture is shown in Figure 15. For example, in this figure $\mathrm{eNB}_{1}$ is elected as the $\mathrm{CS}$ for $\mathrm{UE}_{2}, \mathrm{UE}_{3}$ and $\mathrm{UE}_{5}$. All the three UEs have the same cooperating set $\left\{\mathrm{eNB}_{1}, \mathrm{eNB}_{2}, \mathrm{eNB}_{3}\right\}$. Therefore, after the $\mathrm{CS}$ has been elected all of the three UEs send CSI feedback message to the CS $\left(\mathrm{eNB}_{1}\right)$ only. We also extended the coordination architecture for heterogeneous cellular networks named DCEC-HetNet.

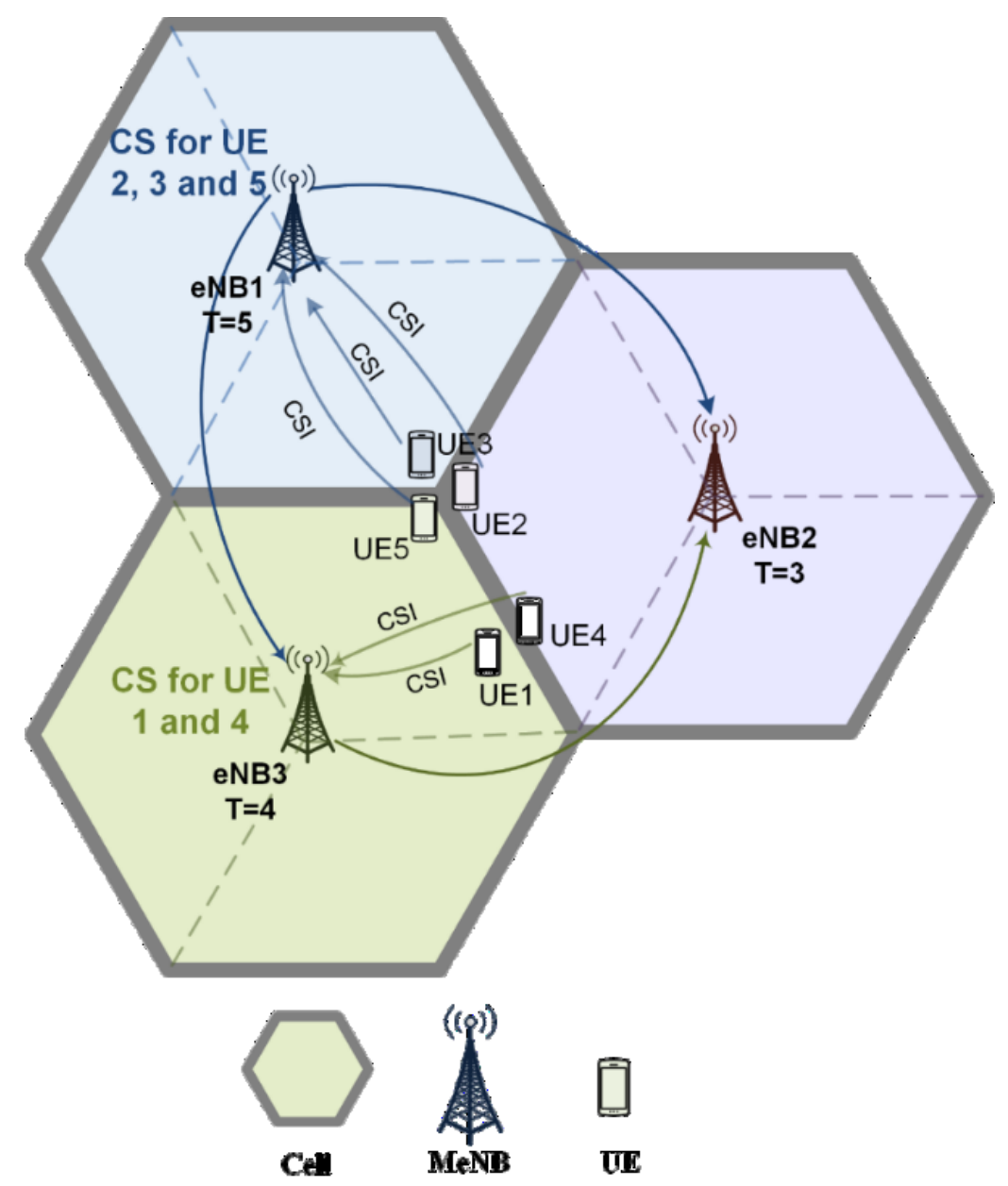

Figure 15: Simplified view of the DCEC CoMP coordination architecture

In order to analyze the performance of the DCEC coordination architecture, we built simulation model and ran simulations of various scenarios suggested by the $3 \mathrm{GPP}$ specifications. We modeled the DCEC, Centralized and Distributed control architectures 
using the discrete event system specifications (DEVS) formalism [29], a formal modeling and simulation methodology for discrete-event dynamic systems. We discussed DEVS briefly in Appendix C.

Our simulation results show that DCEC reduces the number of control message transmitted within the CoMP cooperating set and their feedback latency. Though it requires more control messages to elect the CS in the startup transient period for each UE, under steady state it outperforms the other two architectures (centralized and distributed). In the next subsection, we will discuss DCEC in the context of homogeneous cellular networks.

\subsection{DCEC for Homogeneous Cellular Networks}

The algorithm to elect the coordination station in DCEC CoMP coordination architecture for homogeneous cellular networks is as follows. 


\subsubsection{Coordination Station Election Algorithm for DCEC}

To elect a coordination station (CS) dynamically, we use the following algorithm:

1. The UE estimates the CSI and sends it to the serving MeNB.

2. A serving MeNB receives the CSI Feedback and it calculates the CoMP cooperating set.

3. If a CoMP cooperating set contains more than one MeNB, the serving MeNB declares itself as a CS

4. The declared CS sends a CS-Declaration message to other MeNBs in the set (containing the ID of the sender, the ID of the CS, and the cell throughput of the CS)

5. After receiving the message, other MeNBs in the set compare their throughput with the received CS throughput.

a. If the received CS throughput is higher than the recipient's throughput (or the current):

$i$. The CS ID will change to the received ID.

ii. The recipient forwards the new CS information to the MeNBs in the cooperation set.

b. If the received CS throughput is equal to its own throughput (or the current), and the CS ID is smaller than its own ID (or the current):

$i$. The current CS ID will become the received CS ID.

ii. The recipient forwards the new CS information to the MeNBs in the cooperation set. 
c. If the received CS throughput and ID are equal to the current CS throughput and ID, the CS elected. Stop.

d. Otherwise, the recipient MeNB declares itself as the new CS and sends a CS-Declaration message to the other MeNBs in the CoMP cooperation set.

6. If the cell throughput or cooperating set change, go back to step 3 .

\subsubsection{Signaling Procedure for DCEC in Homogeneous Cellular Networks}

Figure 16 shows the signaling procedure of the DCEC scheme for homogeneous cellular networks. The UE estimates the CSI and feeds it back to its serving macro eNB (MeNB). For example, in Figure 16, the UE1 sends the CSI feedback to MeNB1. After receiving the CSI, MeNB1 calculates the cooperating set for the UE. To calculate this cooperating set, the serving MeNB (MeNB1 for UE1 in this example) compare the channel quality from the received CSI based on the predefined CoMP threshold (3dB-9dB [111, 68, 112]). That is, if $R S R P_{\text {max }}-R S R P_{e N B_{i}} \leq \operatorname{CoMP} P_{T h}$, the $e N B_{i}$ is included into the cooperating set, where, $R S R P_{\max }$ is the maximum received power and $\operatorname{CoMP}_{T h}$ is the predefined CoMP threshold value. If the cooperating set contains more than one MeNB, then the serving MeNB (MeNB1) initiates the election algorithm by sending a CoMP request message to the other MeNBs in the cooperating set (MeNB2, MeNB3), including its own cell throughput. After receiving the CoMP request, they check their own resources and compare the received throughput to their own. 


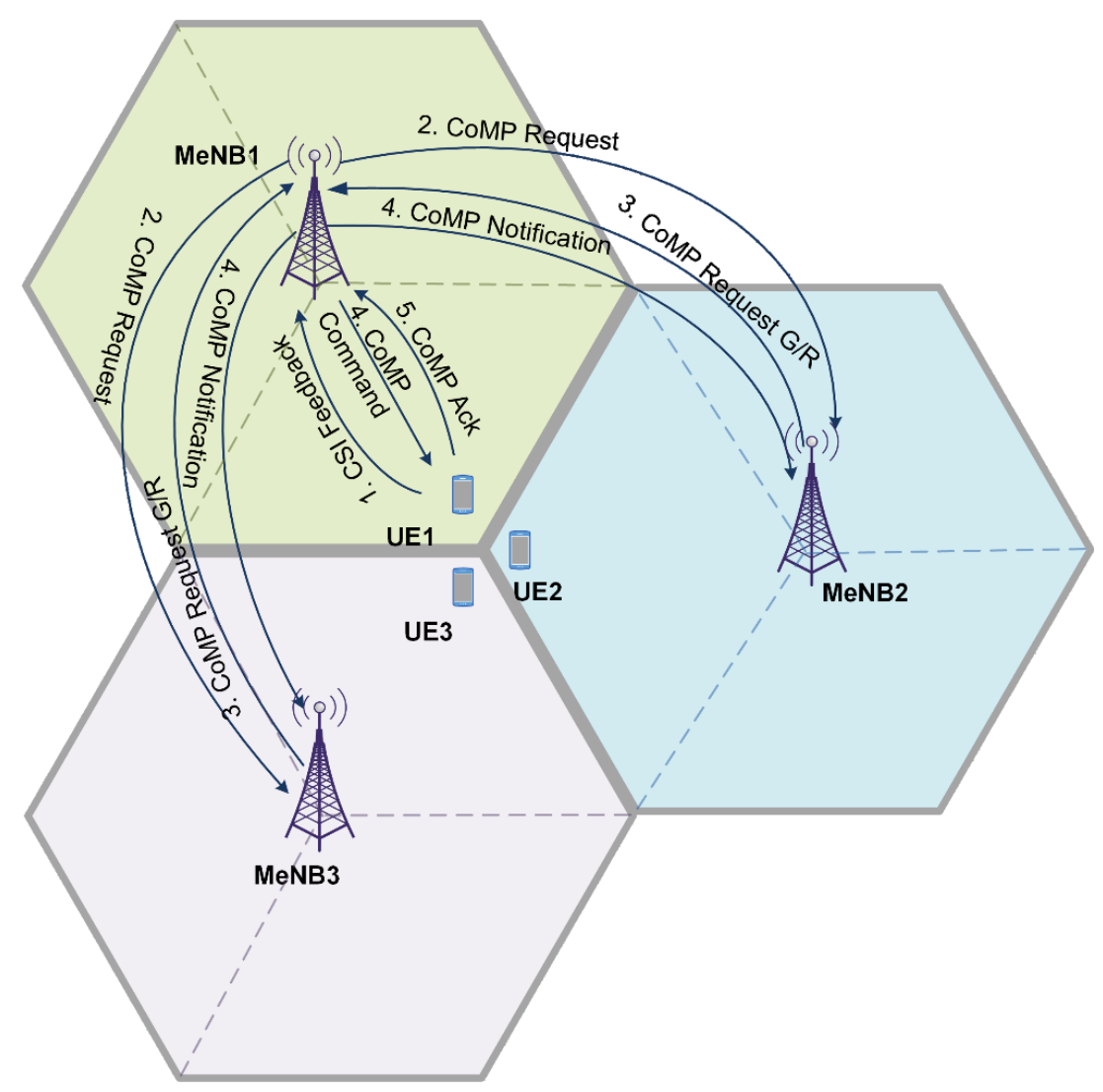

Figure 16: Message transfer to establish CoMP with CS election in DCEC

Based on the availability of resources, they send back a request grant/reject message, including the highest throughput. After receiving the responses, the serving MeNB makes a decision and notifies that to the other MeNBs, sends the CoMP command to the UE (UE1), which replies with an ACK message and switches to CoMP mode. After establishing CoMP with the CS elected, all the UEs in the same CoMP cooperating set send the CSI feedback directly to the same CS, which is in charge of scheduling and radio resource management for the UEs. 


\subsubsection{Modeling the DCEC Architecture Using DEVS}

\subsubsection{Network Architecture}

To study the coordination architecture of the CoMP employing the DCEC algorithm we consider homogeneous networks as suggested by 3GPP specifications in [27]. Figure 17 shows a sample homogeneous network with 3 macrocells. The Macro eNBs within different cells are connected using X2 link. The UEs communicates with each other through the MeNBs using radio frequency (RF). In the simulation scenarios and results section, we discussed different scenarios used in the analysis of simulation outcomes in details. To design simulation model, we consider similar architecture of the network, but the size of the network could be any number of cells according to the coverage area.

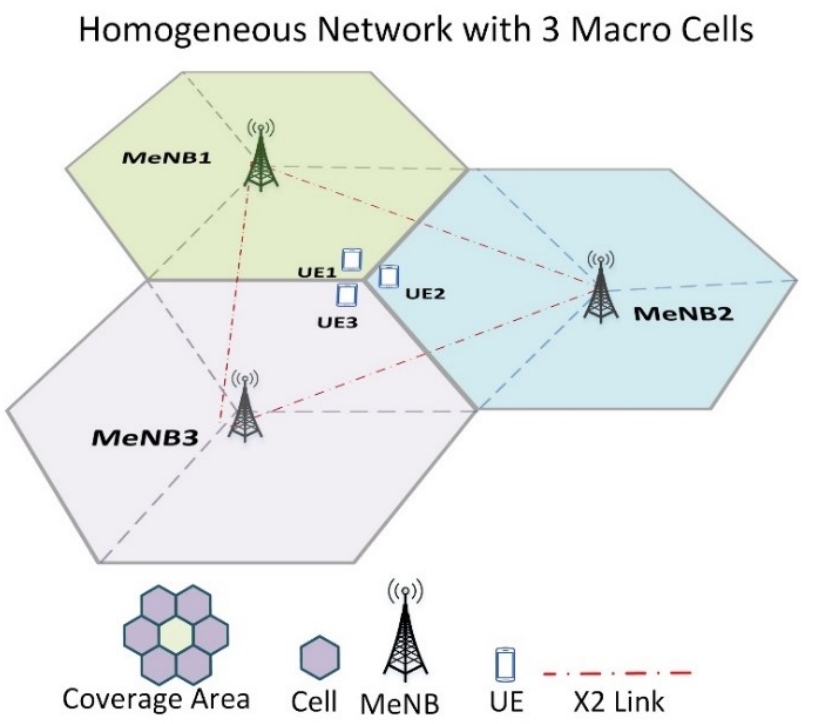

Figure 17: Homogeneous Cellular Network Architecture

\subsubsection{Model Specification}

We designed a DEVS model to examine the performance of CoMP coordination architectures. The high-level structure of the model is shown in Figure 18. 


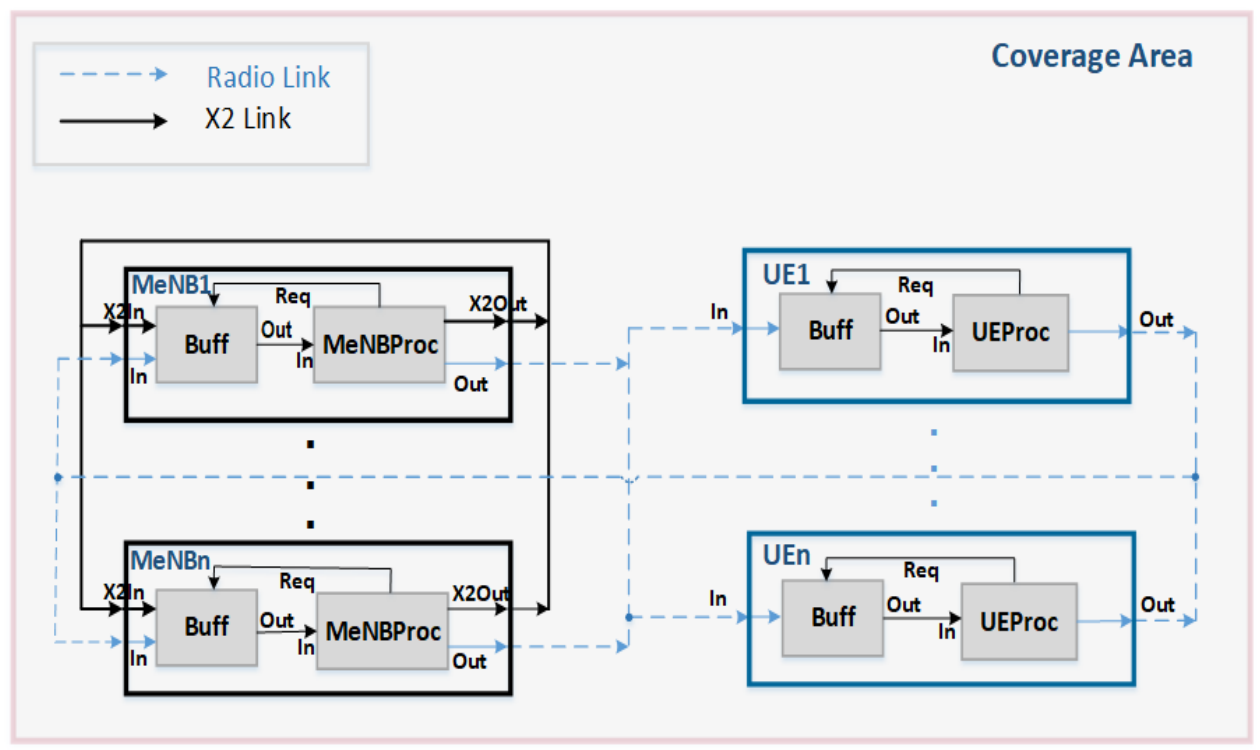

Figure 18: Simplified DEVS model for CoMP coordination architecture in homogeneous cellular networks

The top level coupled model is the CoMP geographic area or coverage area, which includes a number of cells. Each cell contains one macro base station or MeNB and many UEs. The number of UEs and the number of cells vary based on different scenarios. Each MeNB and UE coupled model is composed of two atomic models named Buff and Proc. The UEProc estimates the CSI based on the signal strength received from cooperating MeNBs and feeds it back to the serving MeNB Buff through the output port (Out) periodically. The MeNB Buff atomic model acts as a buffer for the MeNB. Once the MeNB receives a message, the MeNB Buff pushes it in a queue. The message is popped out from the queue and forwarded to the MeNBProc when a request is received from the processor. The MeNBProc sends a request to Buff through Req port. The MeNBProc executes the algorithm discussed earlier in this chapter to calculate a CoMP cooperating set and to elect the CS. In Figure 18, the black solid links connecting the MeNBs represent the X2 links and the blue dotted lines connecting the MeNBs and the UEs represent the radio link. X2 is a point-to-point link 
between two MeNBs. Moreover, the number of MeNBs and UEs can be any number based on the coverage area and the simulation scenarios.

\subsubsection{Model Implementation}

The model is implemented in $\mathrm{CD}++$ toolkit, an open source simulation platform that implements DEVS and Cell-DEVS methodology. Figure 19 below depicts a simplified UML class diagram of the model discussed above. The MeNBProc class implements the MeNBProc atomic model and characterizes with id, position, transmit power, frequency, throughput, etc. The UEProc class implements the UEProc atomic model with the properties such as: id, position, transmit power, frequency, etc. The UEProc class, atomic component, calculates received signal power based on the formula discussed below and sends the CSI feedback periodically to its serving MeNB. Based on the received CSI feedback MeNBProc atomic model calculates the CoMP cooperation set using the formula as discussed in subsection 3.1.2 and elect CS for the UE using the algorithm discussed in Section 3.1.1. 


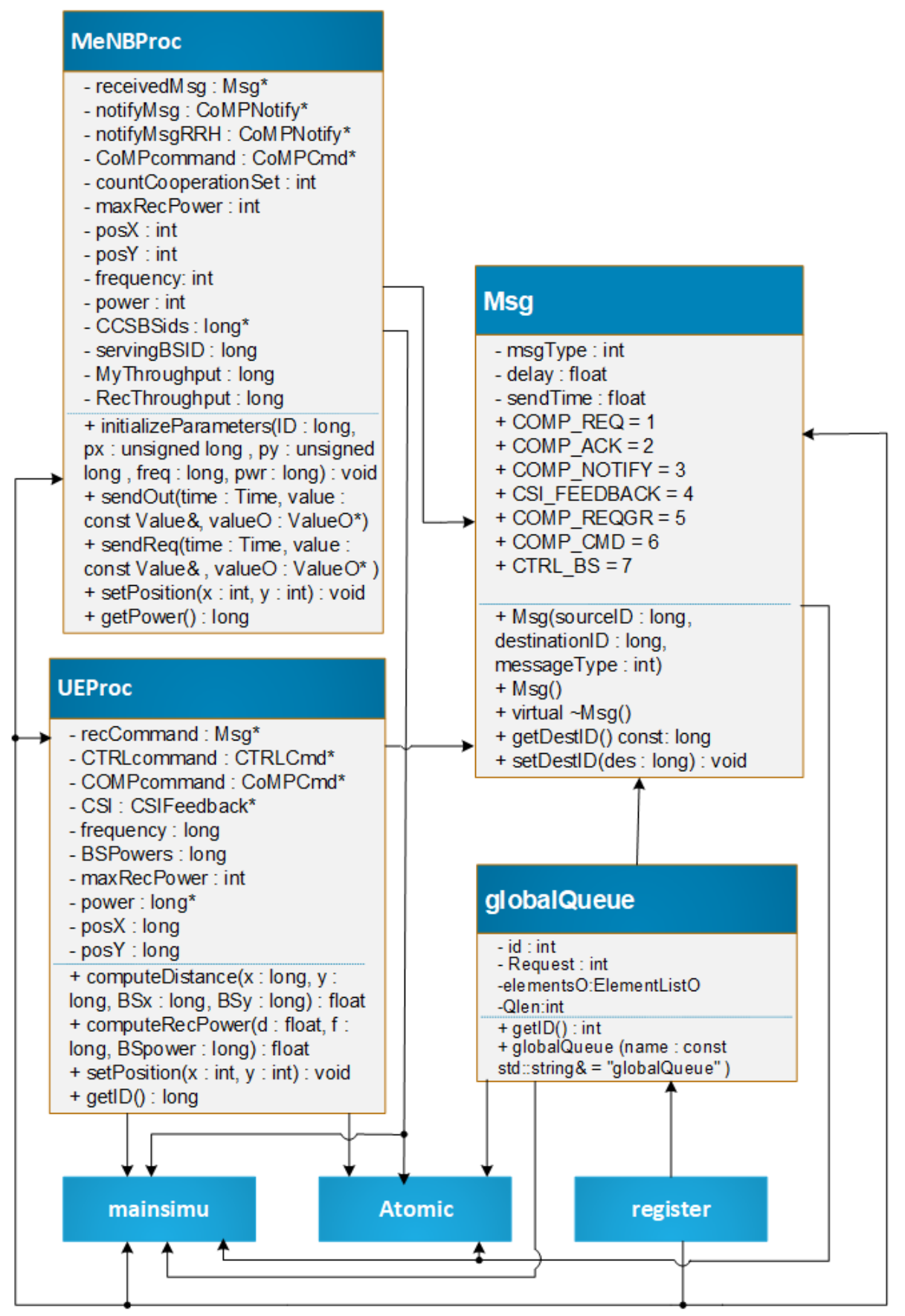

Figure 19: Simplified class diagram of the model for homogeneous networks

We use seven types of messages as shown in Msg class:

- CRTL_BS: MeNBs send system information to UEs. 
- CSI_FEEDBACK: contains the channel state information sent from the UE to the MeNB.

- COMP_REQ: a request message sent from the serving MeNB to other MeNBs in the CoMP cooperation set to join CoMP and elect the CS.

- COMP_REQGR: a grant/reject message sent from the recipient MeNBs to the serving MeNB based on the availability of resources.

- CoMP_COMMAND: a command sent from the serving MeNB to the UE informing about the elected CS and to switch to the CoMP mode.

- COMP_NOTIFICATION: a notification sent from the serving MeNB to other MeNBs within the cooperation set to notify about the formation of CoMP cooperation set and the elected CS.

- COMP_ACK: an acknowledgment corresponding to the CoMP command, sent from the UE to the serving MeNB.

To evaluate the potential of the DCEC CoMP coordination architecture, we ran a series of simulations on this model as discussed in Section 3.4. In the next section we extend the DCEC for heterogeneous networks.

\subsection{DCEC for Dense Heterogeneous Cellular Networks}

Dense heterogeneous networks are considered as a promising technology to cope with the demand for data traffic and providing services to a massive number of users in wireless cellular networks as we discussed in Chapter 2. Low power nodes such as Pico, Femto, and RRH coexist with macro networks to improve coverage and throughput of the networks. However, this coexistence of small cells and macro cells, and the proximity of the access points increase interference, especially for the UEs at the edge of small cells and 
macrocells. This interference causes significant performance degradation of the networks $[113,74]$. As discussed earlier, CoMP can improve the performance of the network by mitigating the interference and serving a UE jointly as well. Here, we show an extended DCEC coordination architecture for heterogeneous cellular networks called DCEC-HetNet [26].

\subsubsection{Coordination Station Election Algorithm for DCEC-HetNets}

In order to select a coordination station (CS) dynamically within the DCEC-HetNet, we use the following algorithm.

1. The UE estimates the CSI and sends it to the serving eNB (MeNB, PeNB or RRH).

2. A serving eNB (MeNB, PeNB or RRH) receives the CSI Feedback form the UE.

3. If an RRH receives the CSI feedback from a UE,

a. RRH forwards the CSIfeedback to the MeNB/BBU it is connected to.

b. The MeNB/BBU calculates the CoMP cooperating set.

Else if serving MeNB/PeNB receives the CSI Feedback

c. MeNB/PeNB calculates the CoMP cooperating set.

4. If a CoMP cooperating set contains more than one MeNBs/PeNBs, the serving $M e N B / P e N B$ in the CoMP set declares itself as a CS.

5. The declared CS sends a CS-Declaration message to other MeNBs/PeNBs in the set (containing the ID of the sender, the ID of the CS, and the cell throughput of the CS)

6. After receiving the message, other MeNBs/PeNBs in the cooperation set compare their throughput with the received CS throughput. 
a. If the received CS throughput is higher than the recipient's throughput (or the current):

- The CS ID will change to the received ID.

- The recipient then forwards the new CS information to the MeNBs/PeNBs in the cooperation set.

b. If the received CS throughput is equal to its own throughput (or the current), and the CS ID is smaller than its own ID (or the current):

- The current CS ID will become the received CS ID.

- The recipient then forwards the new CS information to the MeNBs/PeNBs in the cooperation set.

c. If the received CS throughput and ID are equal to the current CS throughput and ID, the CS has been elected. Stop.

d. Otherwise, the recipient MeNB/PeNB declares itself as the new CS and sends a CS-Declaration message to the other MeNBs/PeNBs in the CoMP cooperation set.

7. If the cell throughput or cooperating set change, go back to step 4.

\subsubsection{Signaling Procedure for DCEC-HetNet}

Figure 20 shows a simplified signaling procedure of the proposed scheme. The UE reports the CSI feedback to its serving MeNB/PeNB/RRH. For instance, in Figure 20, UE1 sends the CSI feedback to its serving RRH11, which forwards the received CSI to MeNB1/BBU1. After receiving the CSI message, eNB calculates the cooperating set for the UE. To do that, it compares the channel quality of neighboring eNBs based on the predefined CoMP threshold $(3 \mathrm{~dB}-9 \mathrm{~dB}[111,68,112])$ and the formula we discussed in 
Section 3.1.2. If the cooperating set contains more than one eNBs, the serving eNB (MeNB1) initiates the election algorithm to select the CS by sending a CoMP request message to the other eNBs (MeNBs and PeNBs) in the cooperating set, such as MeNB2/MeNB3 with its own cell throughput. After receiving the CoMP request, MeNB2/MeNB3 check their own resources and compare the received throughput with their own. Based on the availability of resources, they send back a request grant/reject message, including the highest throughput. After receiving the responses from the other eNBs, the serving eNB (MeNB1) makes a decision about the CS, and it advertises it to the other eNBs (MeNB2 and MeNB3 in this case), RRHs (RRH1) and the UE (UE1) using CoMP notification and CoMP command messages. Finally, the UE replies using the $A C K$ message, and it switches to CoMP mode. After the establishment of CoMP and the CS has been elected, the UE starts sending the CSI feedback only to the CS. 


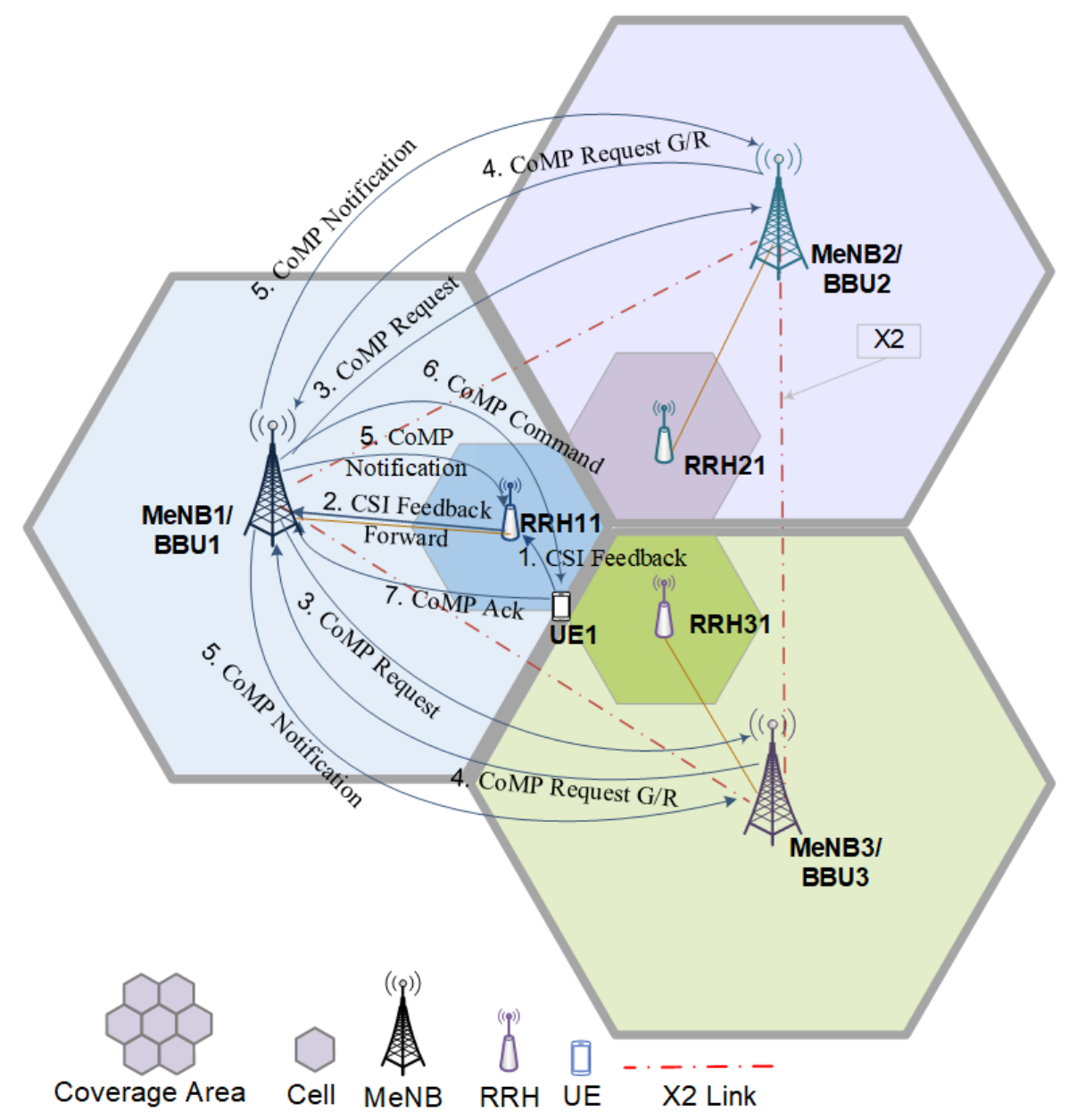

Figure 20: Message transfer to elect CS and establish CoMP in DCEC-HetNet

As previously discussed, all the UEs in the same CoMP cooperating set will send the CSI feedback directly to the same CS only. Therefore, the CSI feedback does not need to travel additional X2, S1 or fiber channels when the UE is in the CoMP cooperation set, which results in avoiding the extra latency of the CSI feedback transmission as well as reducing the signaling overhead into the networks.

\subsubsection{Modeling the DCEC-HetNet Coordination Architecture Using DEVS}

\subsubsection{Network Architecture}

To study the coordination architectures of CoMP in heterogeneous networks employing 
the DCEC-HetNet algorithm we consider the scenarios as suggested by 3GPP in [27]. Figure 21 is a multi-tier heterogeneous networks with 3 macro cells, 1 Pico cell and 3 RRHs. The connections between MeNBs and RRHs are optical fiber, MeNB to MeNB and MeNB to PeNB are X2 links. The numbers of UEs and the number of cells can be any number according to the simulation scenarios. In Section 3.4 we discussed the simulation scenarios and results.

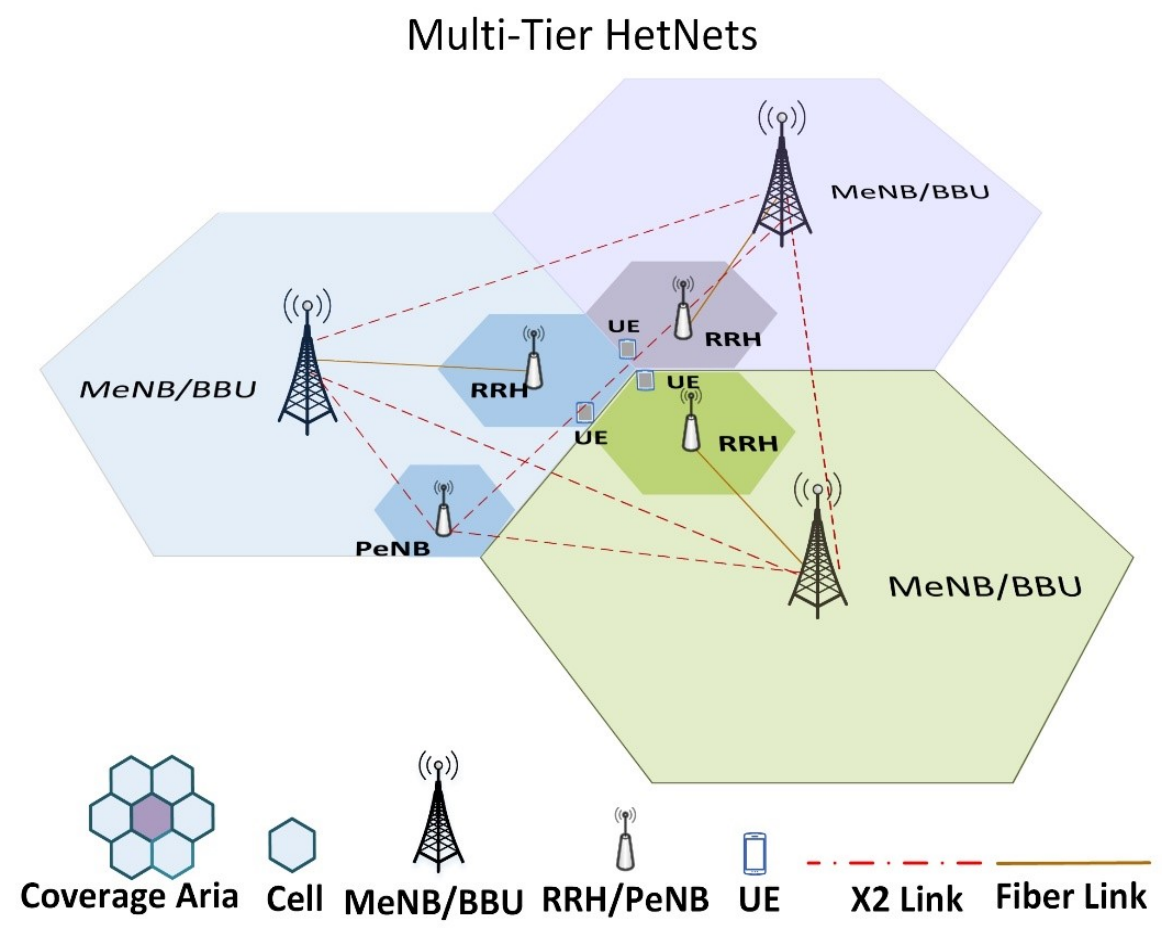

Figure 21: Simplified Network Architecture for HetNets

\subsubsection{Model Specifications}

We designed a DEVS model to examine the performance of CoMP coordination architectures in heterogeneous cellular networks. The high-level structure of the DEVS model is shown in Figure 22. 


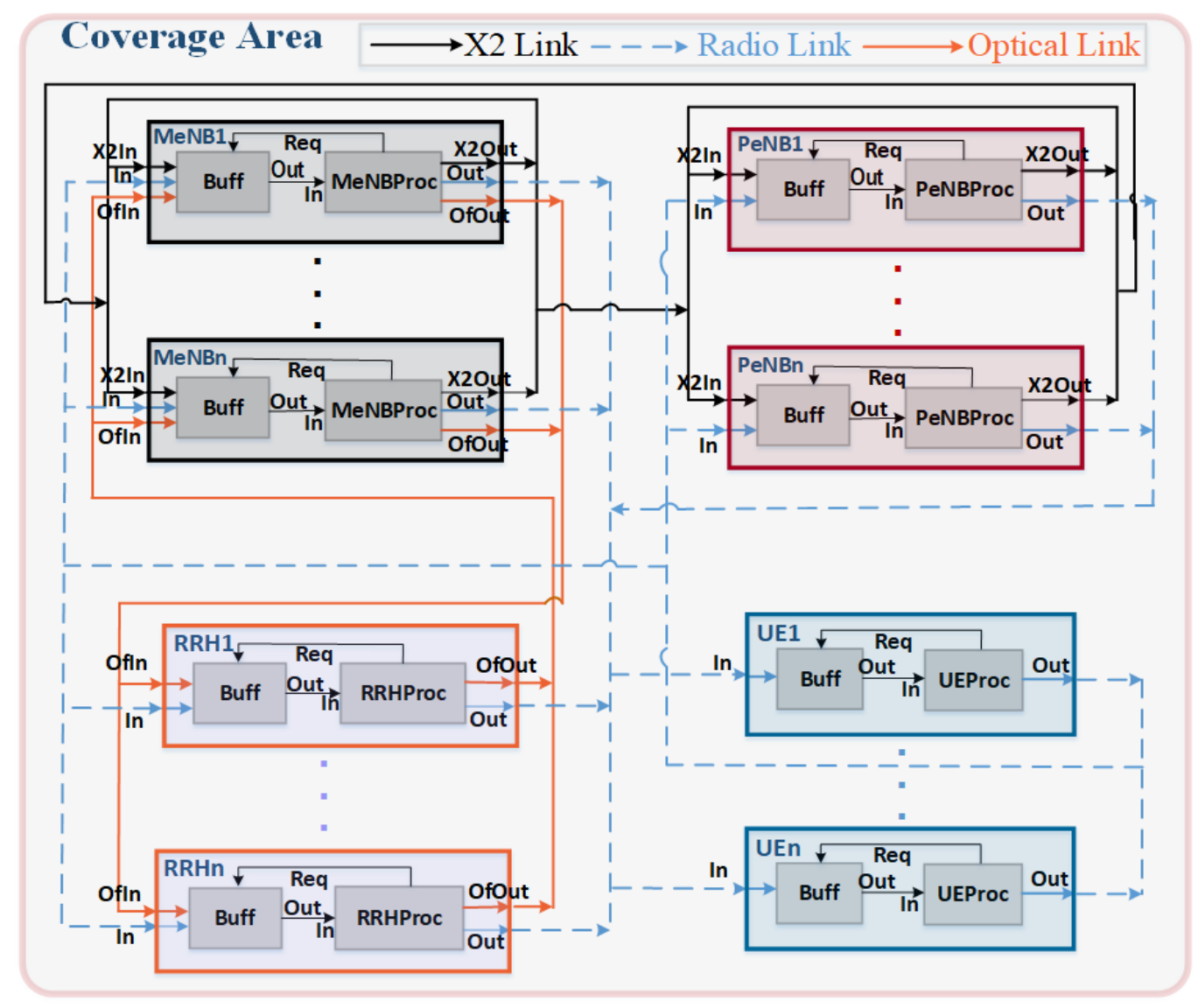

Figure 22: Simplified DEVS model for CoMP coordination architecture in heterogeneous cellular networks

The top level coupled model is the CoMP geographic area or coverage area, which includes a number of cells. Each macro cell contains one MeNB, multiple RRHs or PeNBs and many UEs. The numbers of PeNBs, RRHs and UEs varies based on different scenarios. Each MeNB, PeNB, RRH and UE coupled model is composed of two atomic models named Buff and Proc. The UEProc generates the CSI feedback based on the signal strength received from cooperating MeNBs, PeNBs and RRHs and sends it to the eNB Buff or RRH Buff through the output port (Out) periodically. The Buff acts as a buffer for the MeNB, PeNB, RRH and UE. For instance, once the MeNB receives a message, the MeNB Buff pushes it in a queue. The message is popped out from the queue and forwarded to the 
MeNBProc when a request is received from the processor. The MeNBProc executes the algorithm discussed in the previous section to calculate a CoMP cooperating set and elect a CS. In Figure 22, the black solid links connecting the MeNBs, PeNBs, and MeNBs and PeNBs represent the X2 links, the orange solid lines connecting the MeNBs and the RRHs represent optical links and UEs connect to the MeNBs, PeNBs and RRHs through the radio link. Moreover, the number of MeNBs, PeNBs, RRHs and UEs can be any number based on the simulation scenarios.

\subsubsection{Model implementation}

The model is implemented in $\mathrm{CD}++$ toolkit as we stated before. Figure 23 depicts a simplified UML class diagram of the model. The MeNBProc class, PeNBProc class and the RRHProc class implement the MeNBProc atomic model, PeNBProc atomic model and RRHProc atomic model respectively. MeNBProc and PeNBProc classes are characterized with id, position, transmit power, frequency, throughput etc. The RRHProc class is characterized using its id, position, transmit power, frequency etc. The UEProc class characterises with the properties such as: id, position, transmit power, frequency etc. The UEProc class, atomic component, calculates received signal power based on the formula 13, 14 and 15 discussed later in Section 3.4 and sends the CSI feedback periodically to its serving MeNB, PeNB or RRH. Based on the received CSI feedback MeNBProc or PeNBProc calculates cooperation set and implement CS election algorithm as stated before. The MeNBProc is pretty similar to MeNBProc in homogeneous networks but in this case MeNBProc receives CSI from UE as well as from RRH as a forward message. 


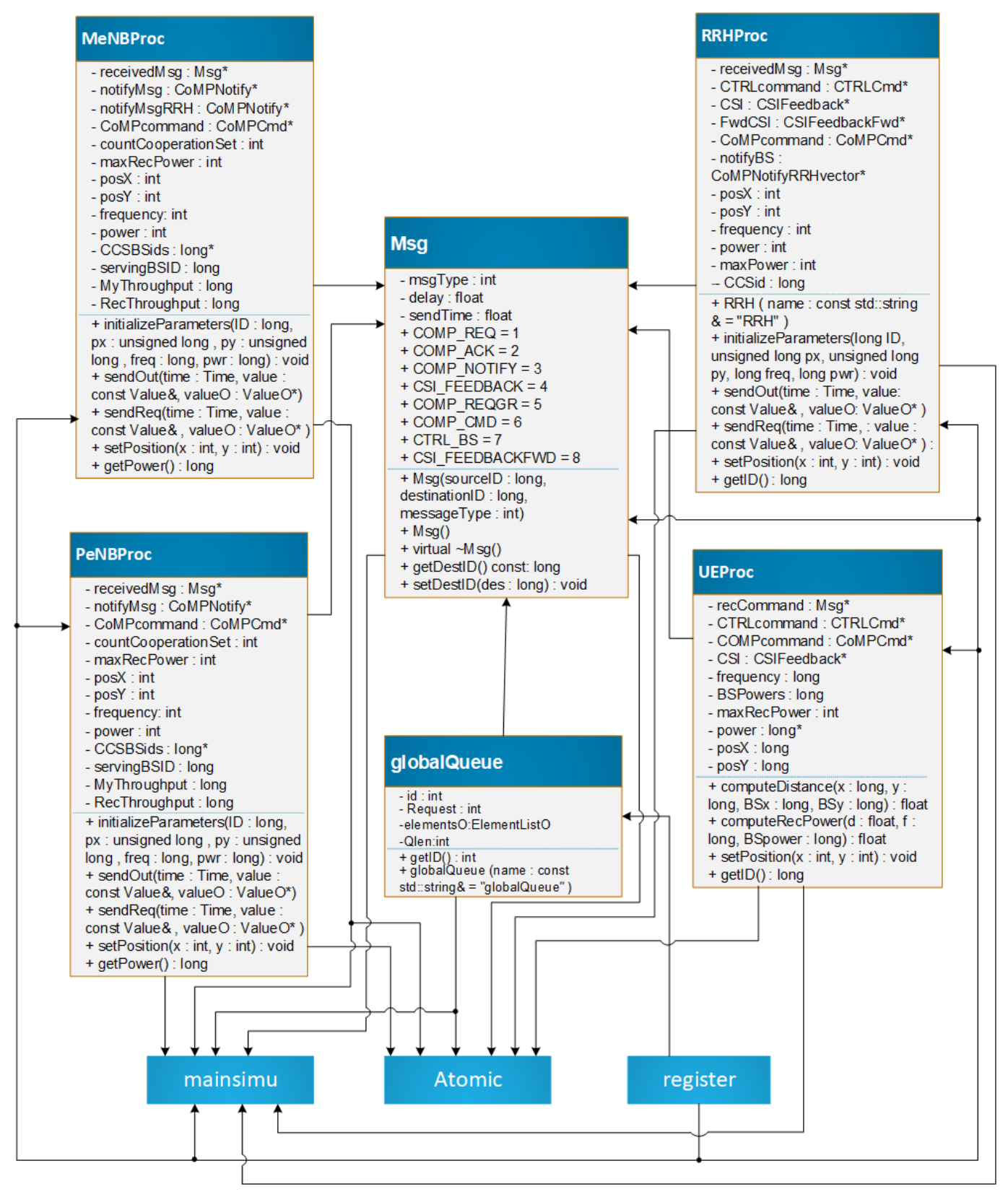

Figure 23: Simplified class diagram of the model

In heterogeneous networks we added one new message type named CSI_FEEDBACKFWD

on top of the messages used in homogeneous networks. RRH uses this message to forward

the CSI feedback received from UE to the MeNB it is connected. All other message types

remain same as homogeneous networks. To evaluate the potential of the DCEC-HetNet 
coordination architecture, we ran a series of simulations on this model, based on the initial conditions summarized in Table 10 in the following section [23, 114, 115].

\subsection{CSI feedback Overhead Estimation}

In coordinated multipoint (CoMP) communication UEs need to estimate the channel state information (CSI) and feed it back to the eNBs as we stated before. The CSI feedback message includes CQI, PMI and RI. The CSI feedback process is discussed in Chapter 2 in details. Four widely recommended codebook-based CSI feedback schemes are wideband, subband, best-M and full feedback [86, 87, 116]. In this section, we derived the CSI feedback overhead model as presented in equation 9 to equation 12 for all of the four feedback schemes based on the 3GPP specification in [116] and the formulas (5 to 8) discussed in Section 2.3.4. We considered all the three components (CQI, PMI and RI) of CSI feedback message for deriving feedback overhead model. Quadrature phase-shift keying (QPSK) modulation technique is considered for all of the feedback schemes.

- Wideband: In wideband scheme, each UE transmits one single 4-bit CQI value in each reported CSI. The 4-bit CQI value describes the channel quality for all of the PRBs in the bandwidth. Therefore, from equation 5 we can derive the CSI feedback overhead model as shown in equation 9.

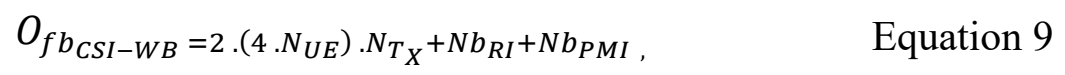

where, $N_{T_{X}}$ is the number of transmit antenna, $N_{U E}$ is the number of UEs served in CoMP operation, $N b_{R I}$ is the bit used for rank indicator (RI) and $N b_{P M I}$ is the allocated bits for PMI reporting in each CSI message. 
- Subband level: The bandwidth is divided into $N_{\text {sband }}$ subbands. The number of consecutive resource blocks in a subband is dependant on bandwidth as shown in Table 6 in Section 2.3.4. In this case, each UE feeds back to the base station one 4 bits wideband CQI and 2 bits differential CQI for each subband. The overhead model for CSI is shown in equation 10 deriving from equation 6 .

$$
O_{f b_{C S I-S B}}=2 .\left(4+2 \cdot N_{\text {Sband }}\right) \cdot N_{U E} \cdot N_{T_{X}}+N b_{R I}+N b_{P M I},
$$

where, $N_{\text {sband }}$ is the number of subbands in the system bandwidth.

- UE selected Best-M: Each UE selects M preferred sub bands of equal size $N_{R B}^{\text {sband }}$ as shown in Table 7 in Section 2.3.4 [116]. In UE selected Best-M scheme, each user feeds back one 4 bits wideband CQI and 2 bits differential CQI to the serving eNB. The 2 bits differential CQI reflects the channel quality only the selected M subbands. In this scheme, UE also report the position of these subbands in the bandwidth. Therefore, from equation 7 we can derive the equation 11 showing the CSI feedback overhead for this feedback scheme.

$O_{f b_{C S I-B M}}=2 \cdot\left(4+2+\left[\log _{2}\left(\begin{array}{c}N_{R B}^{S B} \\ M\end{array}\right)\right]\right) \cdot N_{U E} \cdot N_{T_{X}}+N b_{R I}+N b_{P M I}, \quad$ Equation 11

where, $N_{R B}^{S B}$ is the number of resource blocks in system bandwidth. 
- Full Feedback: In this scheme, each UE reports a 4-bit wideband CQI value and a 2-bit differential CQI for each RB in the system bandwidth. The CSI feedback overhead model for this scheme is in presented in equation 12 .

$$
O_{f b_{C S I-F}}=2 \cdot\left(4+2 \cdot N_{R B}^{S B}\right) \cdot N_{U E} \cdot N_{T_{X}}+N b_{R I}+N b_{P M I} \quad \text { Equation } 12
$$

The number of bits used in reporting rank indicator (RI) is shown in Table 9. Finally, the number of bits used to report PMI is 2 bits and 4 bits for 2 and 4 transmit antenna ports respectively $[116,112,86]$.

Table 9: Number of bits in RI according to the antenna ports

\begin{tabular}{|l|c|c|c|}
\hline & \multicolumn{3}{|c|}{ Antenna ports } \\
\cline { 2 - 4 } & 2 & 4 & $8 / 12 / 16 / 20 / 24 / 28 / 32$ \\
\hline Number of Bits in & 1 & 2 & 3 \\
Rank Indicator (RI) & & & \\
\hline
\end{tabular}

In the next section we presented simulation scenarios and results to analyze performance of the DCEC CoMP coordination architecture.

\subsection{Simulation Scenarios and Results}

In order to study the coordination architectures of CoMP employing DCEC and DCECHetNet, we run a number of simulation scenarios using the different network architectures suggested by 3 GPP [27]. Figure 24 shows a simplified version of the sample simulation scenarios with different layers of cells. Figure 24(a) and 24(b) show homogeneous networks with 3 macrocells and 19 macrocells respectively. Figure 24(c), 24(d) and 24(e) 
shows network architecture of 3 different heterogeneous networks. Figure 24(c) and 24(d) presents scenarios with 3 macrocells with 3 RRHs and 7 macrocells with 19 RRHs respectively. Figure 24 (e) shows a scenario that has 7 macrocells with 70 picocells, 10 picocells in each macro cells. The position of PeNBs are based on the inter site distance (ISD) as mentioned in Table 10. The numbers of UEs varies in different scenarios and discussed in detail later.

To evaluate the potential of the DCEC and DCEC-HetNet coordination architectures, we ran a series of simulations based on the scenarios discussed in Figure 24, using the initial conditions summarized in Table 10 below $[23,114,115]$. The results are obtained by conducting multiple simulation runs for each scenario and considering a $95 \%$ confidence interval. 

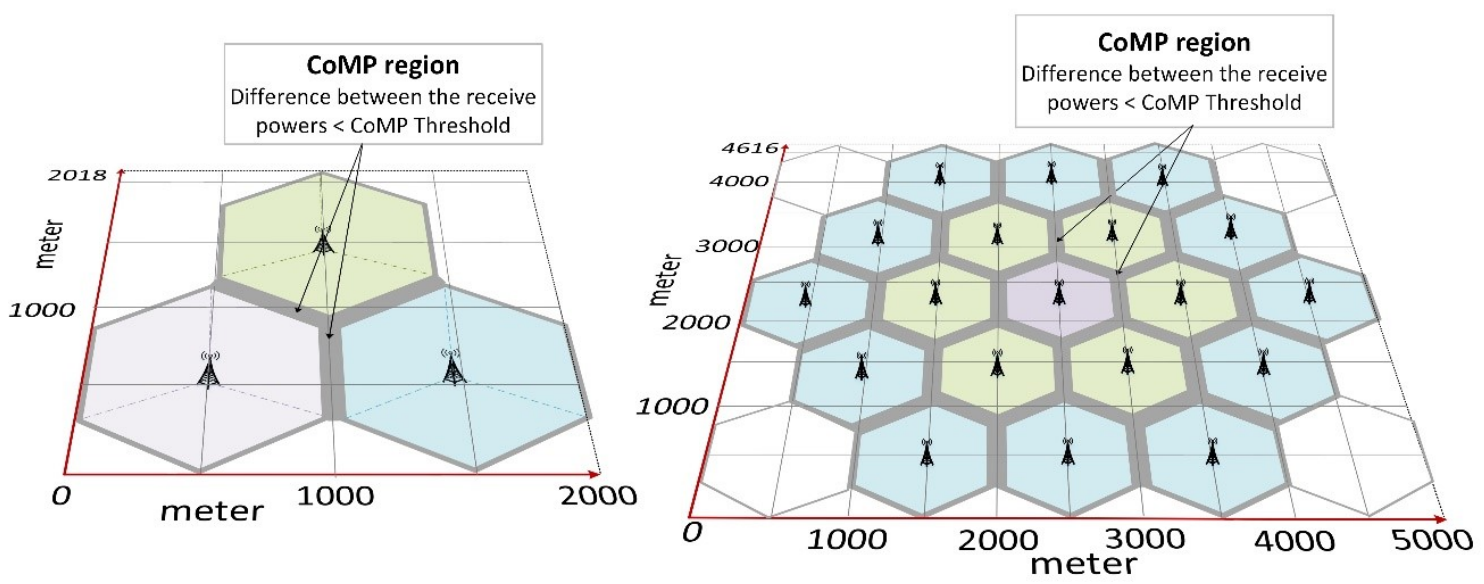

(c) Heterogeneous Network Scenario with 3 Macro (d) Heterogeneous Network Scenario with 7 Macro Cells and $3 \mathrm{RRH}$ Cells and 19 RRH
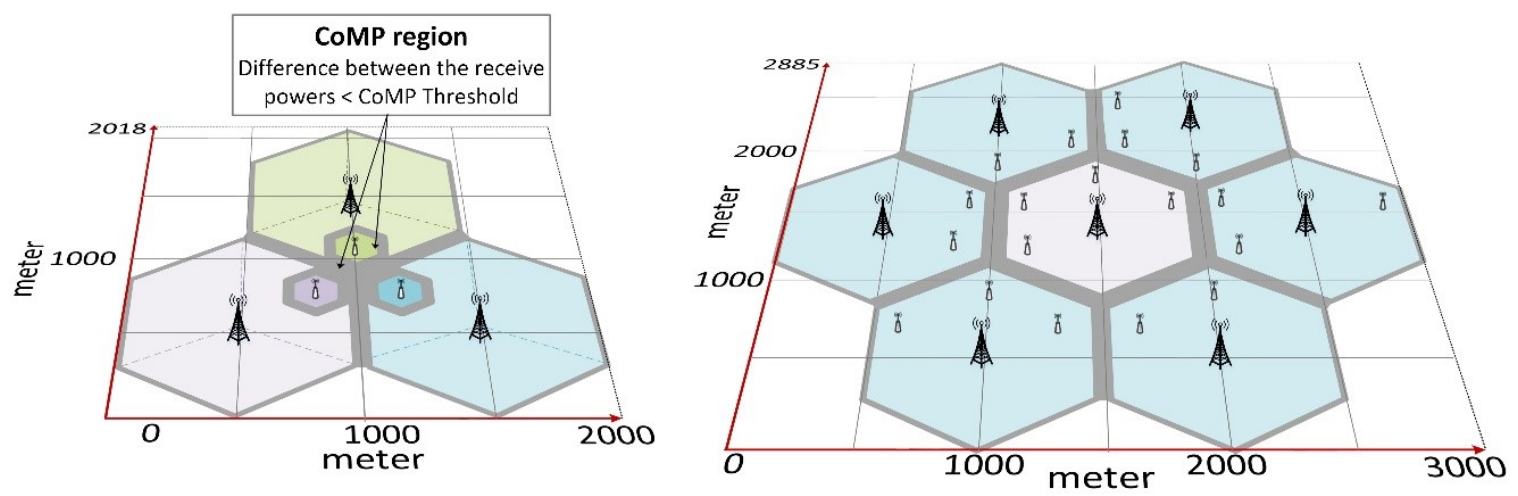

(E) Heterogeneous Network Scenario with 7 Macro Cells and 70 Pico Cells

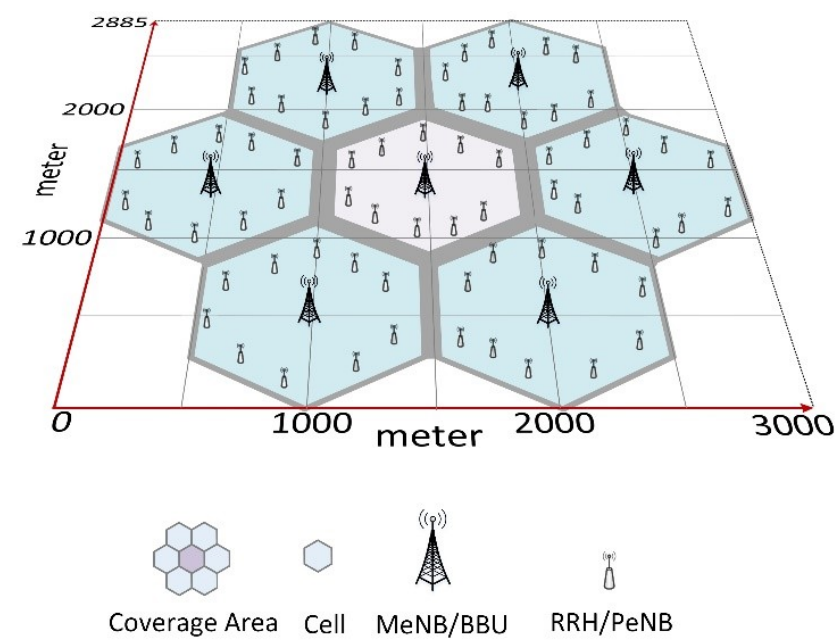

Figure 24: Simulation scenarios considered to evaluate the DCEC and DCEC-HetNet coordination architecture 
Table 10: Simulation assumptions

\begin{tabular}{|l|l|}
\hline \multicolumn{1}{|c|}{ Parameters } & \multicolumn{1}{|c|}{ Values } \\
\hline Number of macro MeNB & 3 and 19 (Homogeneous) \\
& 3 and 7 (Heterogeneous) \\
\hline Number of RRHs in HetNets & 3 and 19 \\
\hline Number of PeNB in HetNets & 70 \\
\hline Density of active UEs in Macro only networks & $2 / \mathrm{km}^{2}, 4 / \mathrm{km}^{2}, 6.5 / \mathrm{km}^{2}$ and $9 / \mathrm{km}^{2}$ \\
\hline Density of active UEs in HetNets & $6 / \mathrm{km}^{2}, 11.5 / \mathrm{km}^{2}, 17 / \mathrm{km}^{2}$ and $23 / \mathrm{km}^{2}$ \\
\hline UE Spatial Distribution & Uniform random distribution in the \\
\hline UE arrival and leave & CoMP area \\
\hline Frequency & Uniform random and Poisson \\
\hline eNB Transmit Power & $2000 \mathrm{MHz}$ \\
\hline RRH Transmit Power & MeNB: $43 \mathrm{dBm}$ and PeNB: $30 \mathrm{dBm}$ \\
\hline Macro Cell Radius & $30 \mathrm{dBm}$ \\
\hline Antenna gain & $500 \mathrm{~m}$ \\
\hline MCL & $12 \mathrm{dBi}(\mathrm{BS}), 05 \mathrm{dBi}$ (RRHs) and $0 \mathrm{dBi}$ \\
\hline LogF & (UEs) \\
\hline Cell Throughput & $70 \mathrm{~dB}$ \\
\hline CSI Feedback periodicity & $10 \mathrm{~dB}$ \\
\hline CoMP Threshold & Uniform: randomly generated \\
\hline & $6 \mathrm{~dB}$ and $10 \mathrm{~ms}$ \\
\hline
\end{tabular}


In our simulation scenarios, we consider homogeneous and heterogeneous networks in an urban area. The transmit power for a MeNB is $43 \mathrm{dBm}$, PeNB is $30 \mathrm{dBm}$ and the RRH is also $30 \mathrm{dBm}$, as suggested in $[26,114,117]$. The received signal power at each UE is calculated based on the following formulas [114]:

$$
P_{r}=P_{t}-\operatorname{Max}\left(L_{\text {path }}-G_{t}-G_{r}, M C L\right),
$$

where $P_{r}$ is the received signal power, $P_{t}$ is the transmitted signal power of the eNBs, $L_{p a t h}$ is the path loss, $G_{t}$ is the transmitting antenna gain and $G_{r}$ is the receiver antenna gain. The minimum coupling loss (MCL) is considered to be $70 \mathrm{~dB}$ [114]. $L_{\text {path }}$ is calculated as follows:

$$
L_{\text {path }}=L+\log F \text {, }
$$

where $\mathrm{L}$ is calculated based on the following formula as suggested by $3 \mathrm{GPP}$ in [114]:

$$
\begin{gathered}
\mathrm{L}=40 \cdot\left(1-4 \cdot 10^{-3} \cdot B_{h}\right) \cdot \log _{10}(d)-18 \cdot \log _{10}\left(B_{h}\right)+\quad \text { Equation } 15 \\
\text { 21. } \log _{10}(f)+80 d B,
\end{gathered}
$$

where $B_{h}$ is the eNB height, which we considered to be 15 meters, $d$ is the separation between $\mathrm{UE}$ and eNB and $f$ is the carrier frequency.

The UEs calculate the received power based on the above formula, generate a CSI feedback message, and send it to the respective eNBs. In our simulation, the MeNBs and PeNBs generate the cell throughput to select the CS at random. Based on the literature in this area, we considered the CoMP threshold of $6 \mathrm{~dB}[111,68]$. 
The simulation outcome presented in Figure 25 shows the number of control messages related to the CoMP download transmission that traveled from the UEs to the CS/MeNBs and the backhaul in specific time intervals, for all the three coordination architectures. Here we consider homogeneous networks.
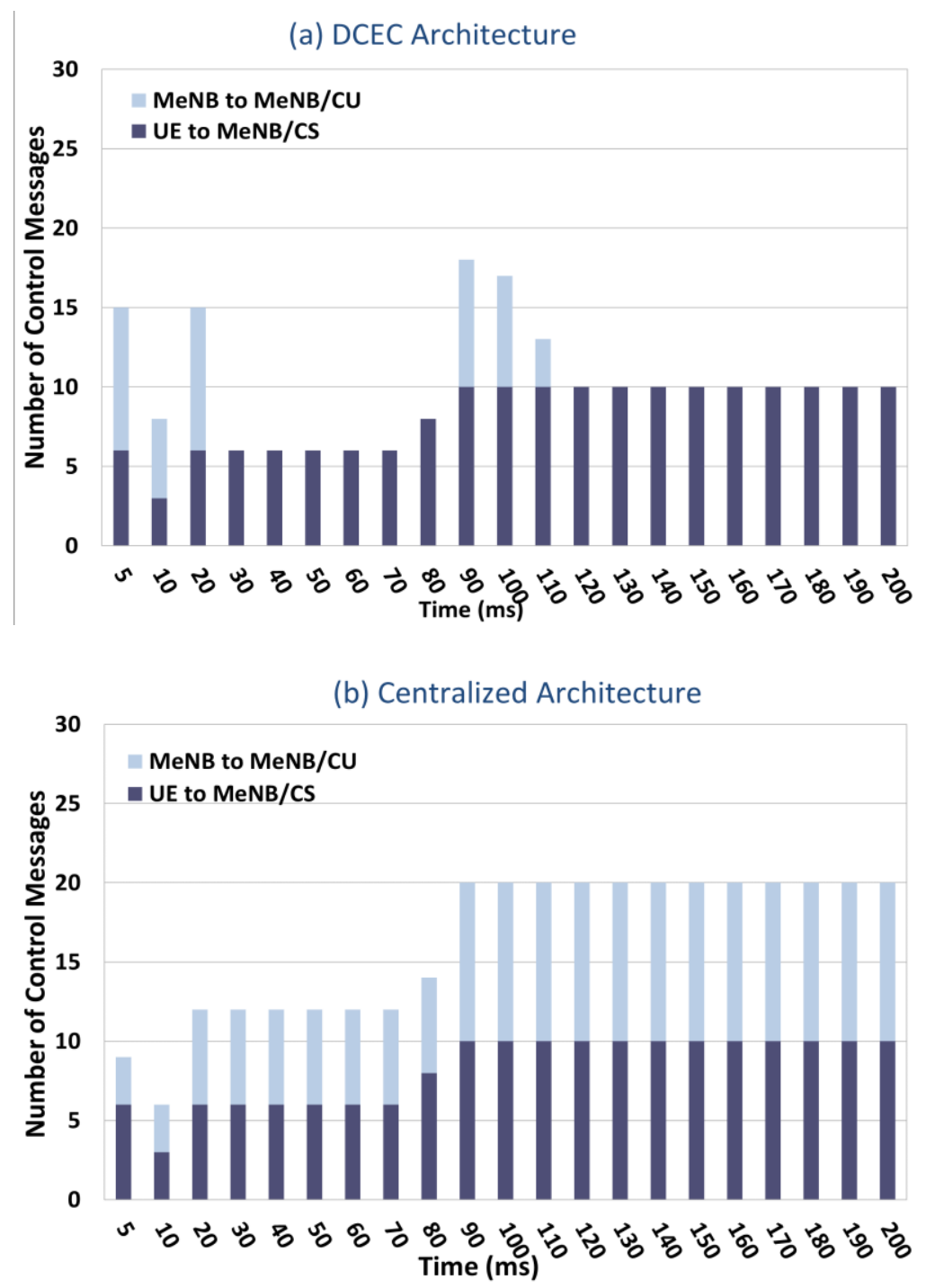


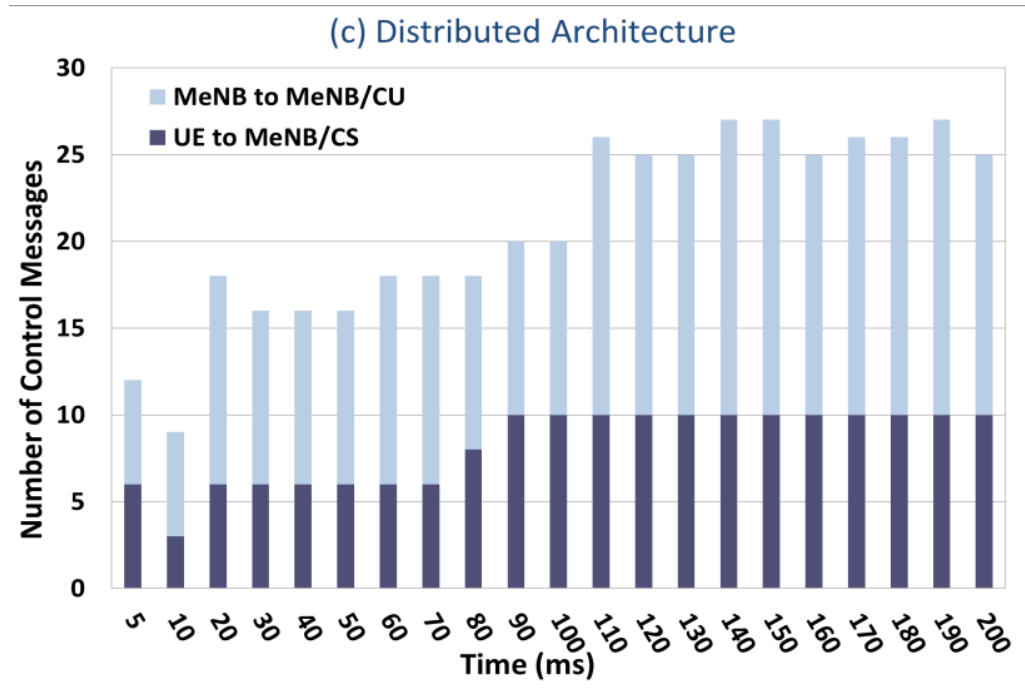

Figure 25: Number of control messages at different time intervals for DCEC, Centralized and Distributed architectures: messages over the backhaul and UE to MeNB

The darker part of each bar in the figure shows the number of CSI feedback messages which traveled from UE to MeNB. The lighter part shows the CSI feedback forwards from MeNB to MeNB, MeNB to CU and the overhead related to the election algorithm. Each bar represents $10 \mathrm{~ms}$ of simulated time, except for the first two groups, which are $5 \mathrm{~ms}$ each. In this scenario, the UEs send the CSI feedback to their serving MeNB or CS every 10ms. The results of the simulations show that in the transient period when we establish CoMP, DCEC needs more control messages over the backhaul; but after the CS has been elected there are no control messages transmitted except for the CSI feedback from the UE to the CS. Because in the DCEC architecture after CoMP established and CS elected all of the UEs in the CoMP transmission send CSI to the CS only. As it is seen in Figure 25(a), no additional control messages are transmitted from MeNB to MeNB within the $30 \mathrm{~ms}$ to $80 \mathrm{~ms}$ timeframe in DCEC. From $80 \mathrm{~ms}$ to $110 \mathrm{~ms}$, there are several new UEs joining the CoMP set, which results in additional control messages transmitted from the UEs to the MeNBs, 
as well as from MeNB to MeNB (to elect the CS). Likewise, from 120ms, there are no additional control messages transmitted through the X2 interface in DCEC (since the CS has been selected). As shown in Figure 25(b) and 25(c), in the other two conventional architectures (centralized and distributed) the CSI feedback needs to be forwarded over the backhaul every time. This increases the signaling overhead as well as the feedback latency into the CoMP networks. The X2 latency is about10ms to $20 \mathrm{~ms}[96,118]$. In most practical systems, the CSI feedback latency consists of processing time, transmission time and waiting time for the scheduler [119]. Here, we consider the feedback delay as the total time between measuring the CSI at the UE and using at the scheduler. In DCEC, as we seen in Figure 25(a), CSI feedback does not need to transmit through X2 interface it also reduces feedback latency. Therefore, according to the simulation results (Figure 25), DCEC reduces the signaling overhead and CSI feedback latency compared to the other two coordination architectures (centralized and distributed). 


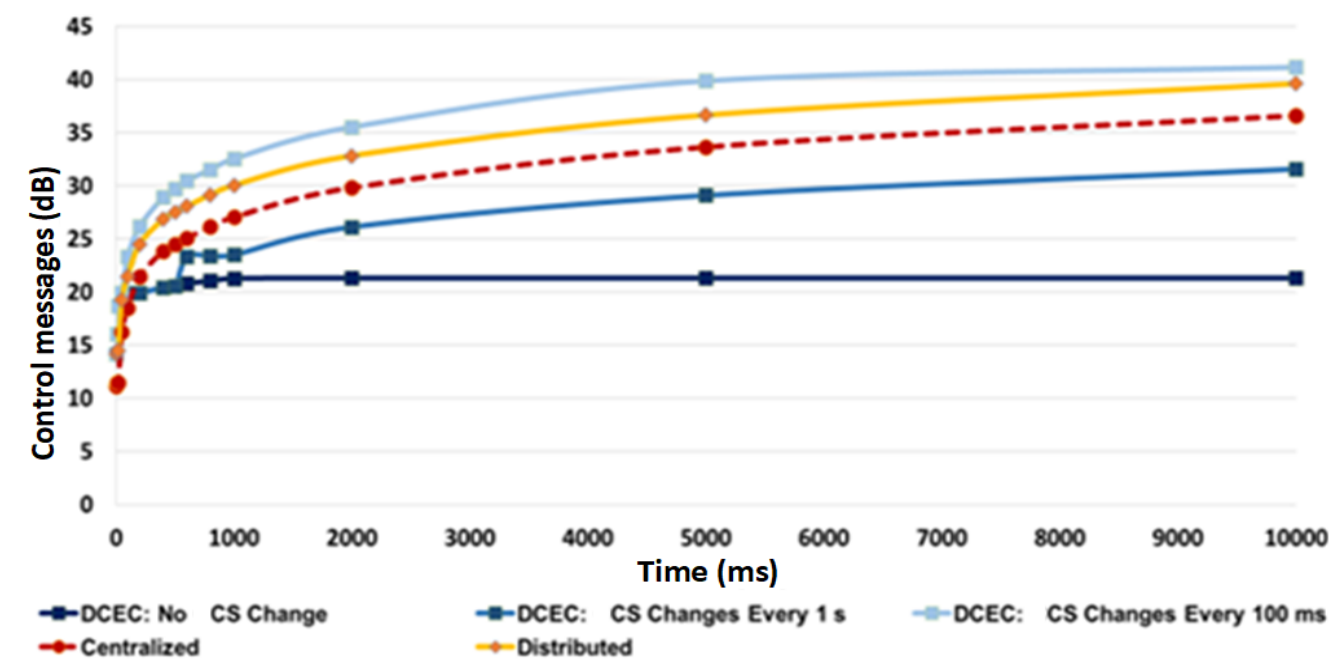

Figure 26: Cumulative control messages for DCEC without CS change, DCEC with CS change every 1s/100ms, Centralized, and Distributed Architectures

Figure 26 shows the cumulative number of control messages transmitted up to a certain time for each of the architectures in the $\mathrm{dB}$ scale. DCEC architecture is represented by three instances to see how the CS changes affect the number of control messages transmitted in the networks. In the first case, we assume that the throughput is constant, that is, the CS does not change it's throughout in the simulation time. In the second and third cases, the CS is set to change every $100 \mathrm{~ms}$ and every $1 \mathrm{~s}$ respectively.

As we can see, DCEC with no CS changes or with changes every 1s outperform the centralized and distributed architectures. If the CS change occurs very rapidly, for example, every $100 \mathrm{~ms}$ or less, DCEC will be less efficient than the traditional approaches. Therefore, if the rate of the CS changes is very high, DCEC will perform worse than the centralized and distributed architectures. However, in practice, the CS change does not occur that 
frequently for most of the UEs since the maximum movement speed of a UE suggested by the $3 \mathrm{GPP}$ in release 11 and 14 for CoMP deployment is $3 \mathrm{~km} / \mathrm{h}[12,27]$.

For further evaluation of the DCEC architecture, we extended the homogeneous simulation scenarios to include 19 macrocells, different density of active UEs within the CoMP operation, and a longer simulation time. The UEs were set to join CoMP based on a Poisson distribution within a 12-hour period (6 $\mathrm{AM}$ to $6 \mathrm{PM})$ with the peak rate at $10 \mathrm{AM}$, as suggested in [120]. As stated earlier, the factors that affect the download and upload performance resulting in a change in user experience are the signaling overhead and latency in CoMP networks. The results are further analyzed for the comparison of the number of CSI feedback messages and delay. Figure 27 shows that using DCEC, the number of feedback messages can be reduced significantly, resulting in better throughput. 

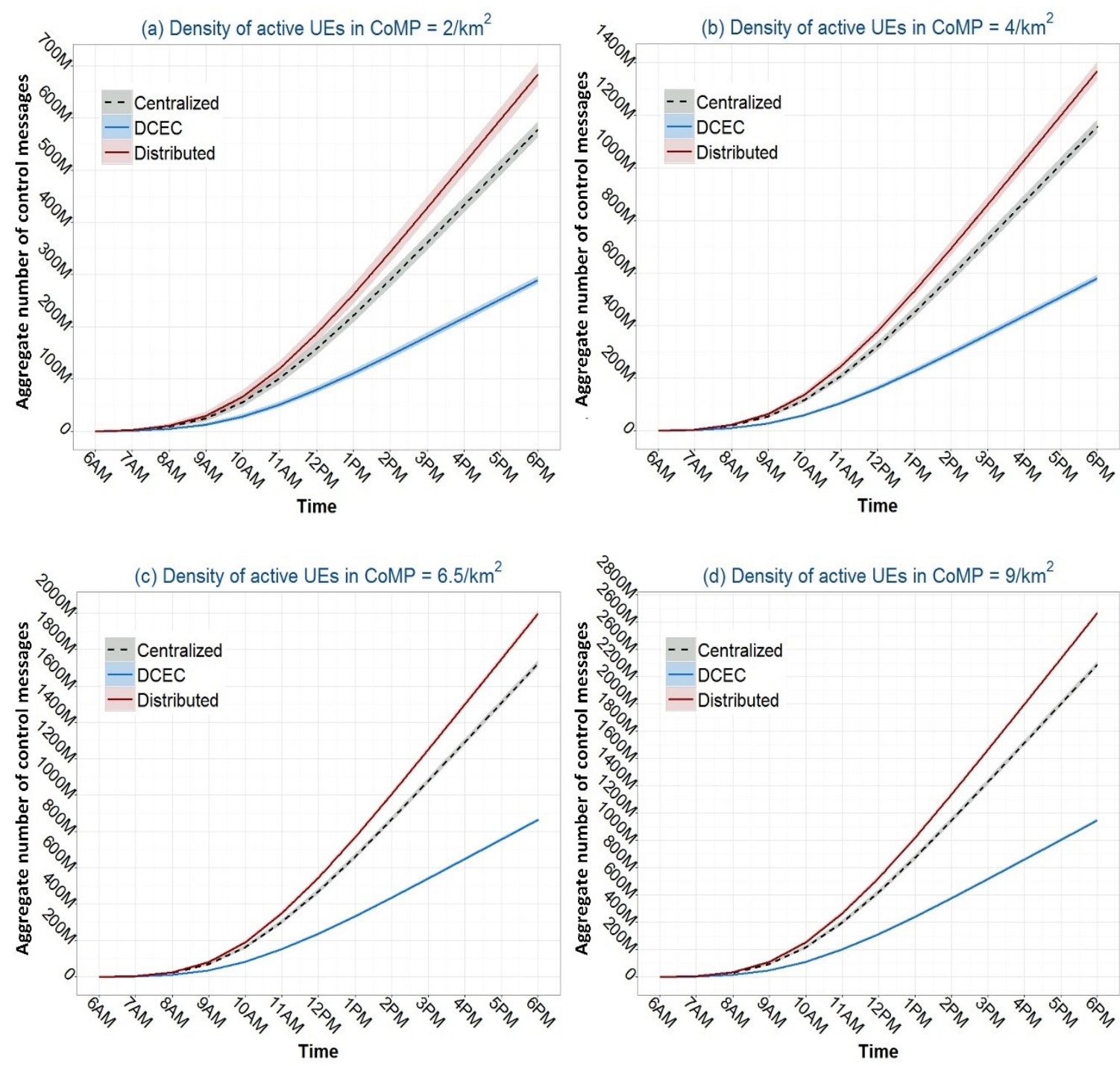

Figure 27: Cumulative number of messages for Centralized, DCEC and Distributed based on the density of the active UEs into CoMP in macro only networks

As seen in Figure 27, DCEC reduces the CSI feedback messages in the network about 50\%. This is because after an eNB is elected to act as the coordination station (CS), eNBs do not need to exchange CSI feedback messages among them. This reduces the signaling load and the possibility of outdated CSI messages that eventually increase the upload and download rate of CoMP operation. 
We also calculated the number of CSI feedback messages sent in every 0.3 minutes timeframes as shown in Figure 28 considering bursty arrival of UEs [9]. This allows us to investigate further the overhead imposed on the network by running DCEC.
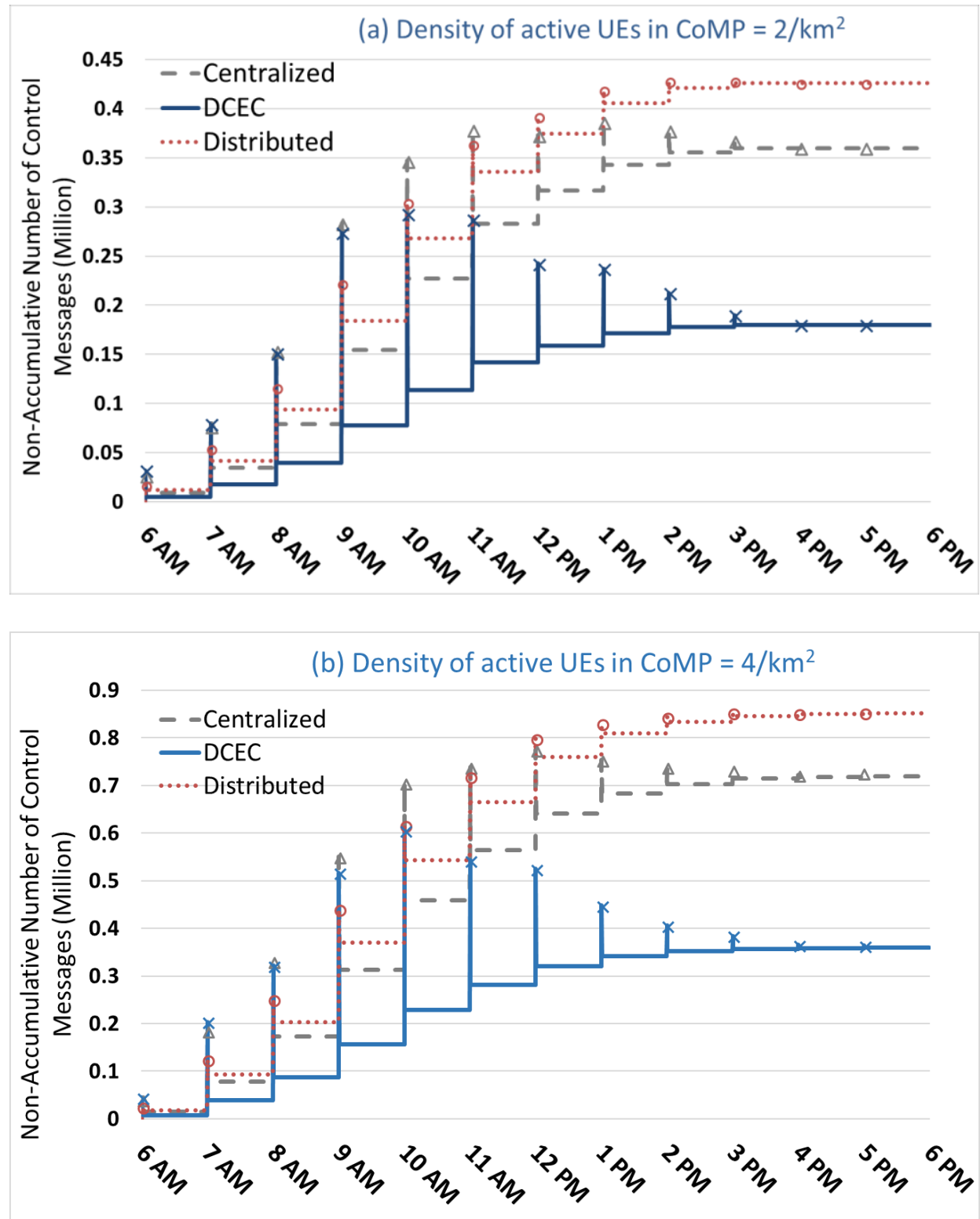


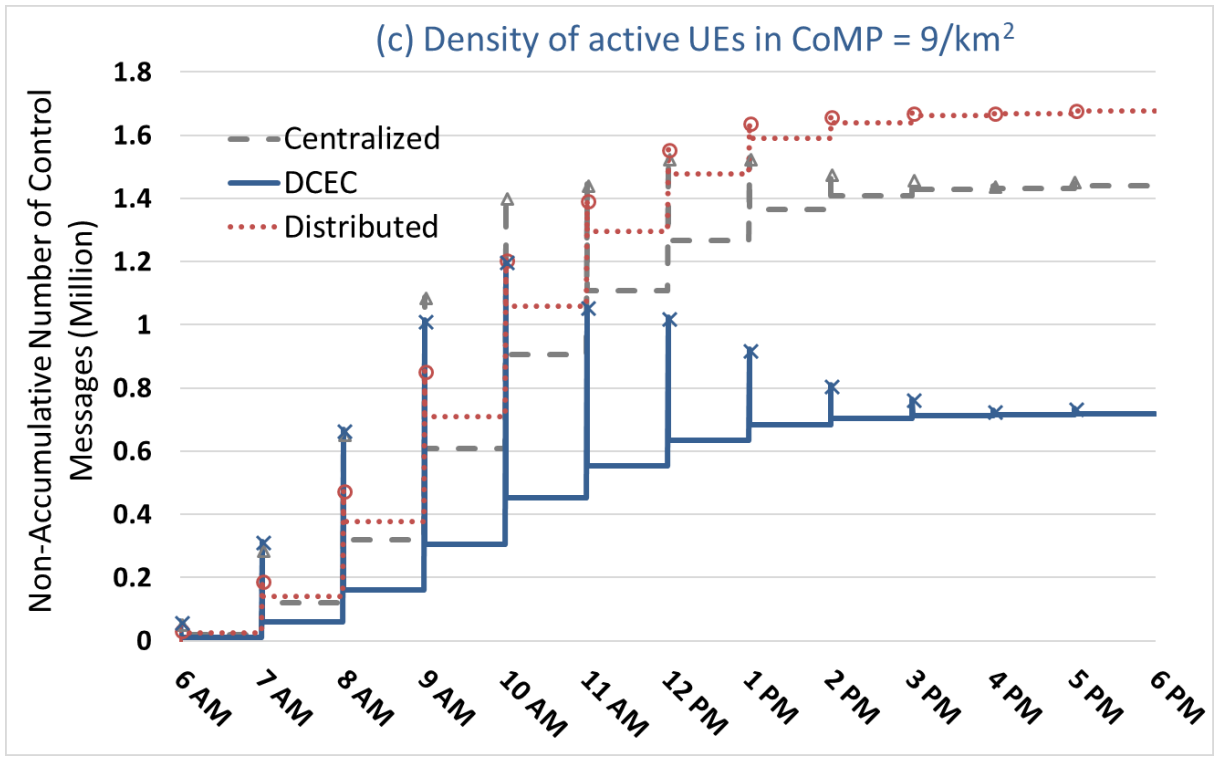

Figure 28: Non-Accumulative number of messages for bursty arrival of UEs with density

$$
\text { of (a) } 2 / \mathrm{km}^{2} \text {, (b) } 4 / \mathrm{km}^{2} \text {, (c) } 9 / \mathrm{km}^{2}
$$

As seen in Figure 28, DCEC performs badly when new UEs join as it is executing the election algorithm whereas the other two algorithms do not need such action. As time goes by, the DCEC approach outperforms the other two approaches. Furthermore, by comparing the density of UEs, it can be seen that DCEC approach is less sensitive to the joining of extra UEs in CoMP.

To evaluate the DCEC-HetNet, in Figure 29, we show a comparison among the CoMP architectures (DCEC-HetNet, centralized and distributed) with respect to the number of control messages in the network as a function of the number of UEs for heterogeneous networks. In this case, 3 MeNBs, 3 RRHs and a different number of UEs (50, 100, 150 and 200 UEs in the entire network) are simulated for $700 \mathrm{~ms}$. By increasing the number of UEs in the HetNet CoMP session, the required control messages increase slower in DCECHetNet than the other two approaches. Therefore, if the number of UE increases in the 
network, the DCEC-HetNet needs fewer control messages to travel within the network compare to other two architectures.

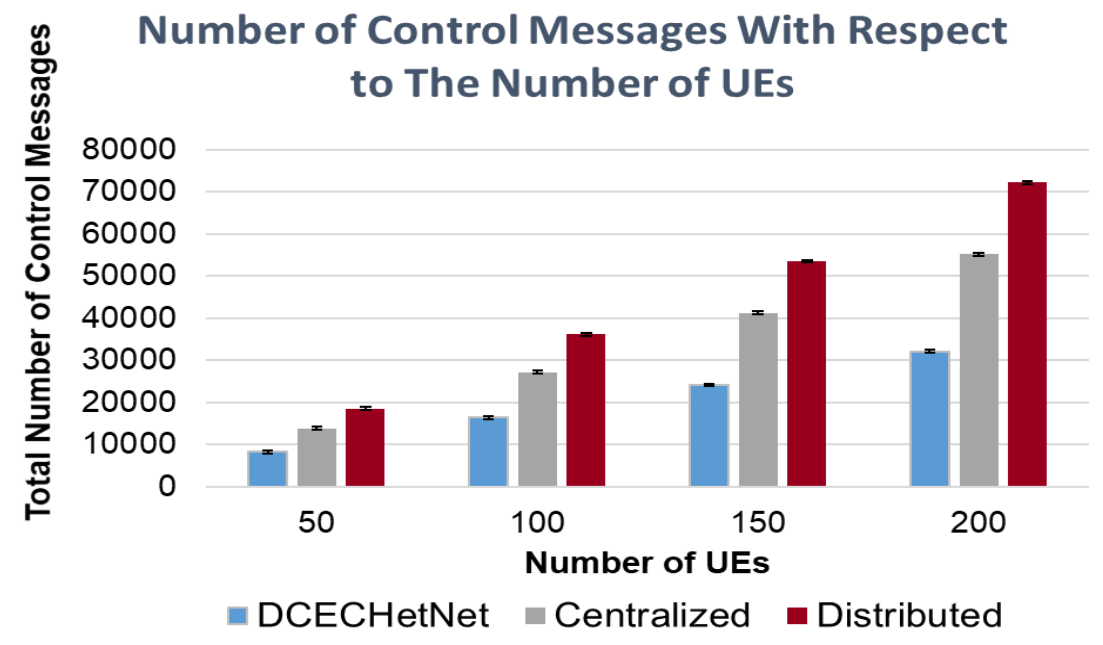

Figure 29: DCEC-HetNet, Centralized, and Distributed architectures based on the number of control messages in the network with respect to the number of UEs in $700 \mathrm{~ms}$

Figure 30 shows the number of control messages traveled into the heterogeneous networks in each $10 \mathrm{~ms}$ time intervals for all the three architectures with 200 UEs in CoMP cooperation. In this figure, the three bars in every group represent the three architectures in the following order: DCEC-HetNet, Centralized and Distributed. This has been shown in the upper left part of the figure. The darker part of each of the bar shows the number of CSI Feedback messages travel from UE to eNB, UE to CS and UE to RRH. The lighter part shows the CSI feedback forwards from MeNB to MeNB, MeNB to CU and the overhead related to the election algorithm. The results of the conducted simulations show that at the beginning of the establishment of CoMP, the DCEC-HetNet control architecture requires additional control messages to be sent over the backhaul, but after the CS has been elected there will be no additional control messages required to be transmitted except the CSI feedback from the UE to the CS. As clearly seen in Figure 30, no additional control 
messages transmitted from MeNB to MeNB within the $20 \mathrm{~ms}$ to $120 \mathrm{~ms}$ (inclusive) timeframe in DCEC-HetNet. In the 130ms to $140 \mathrm{~ms}$ timeframe, several new UEs join the CoMP which results in some additional control messages being transmitted through the backhaul to elect the CS for the new UEs. Again, from time 140ms, there are no additional control messages required since the CS election has been completed. On the other hand, the other two conventional architectures (centralized and distributed) need the CSI feedback to be forwarded over the backhaul every time. Therefore, according to the results of the simulation, as seen in Figure 30, we can see that the DCEC-HetNet architecture reduces the CSI feedback latency compared to the other two control architectures since in this case CSI feedback does not need to travel MeNB to MeNB or MeNB to CU through $\mathrm{X} 2$, S1 or fiber link after the CS elected. The X2 latency is about $10 \mathrm{~ms}$ to $20 \mathrm{~ms}$ as we stated before $[96,118]$.

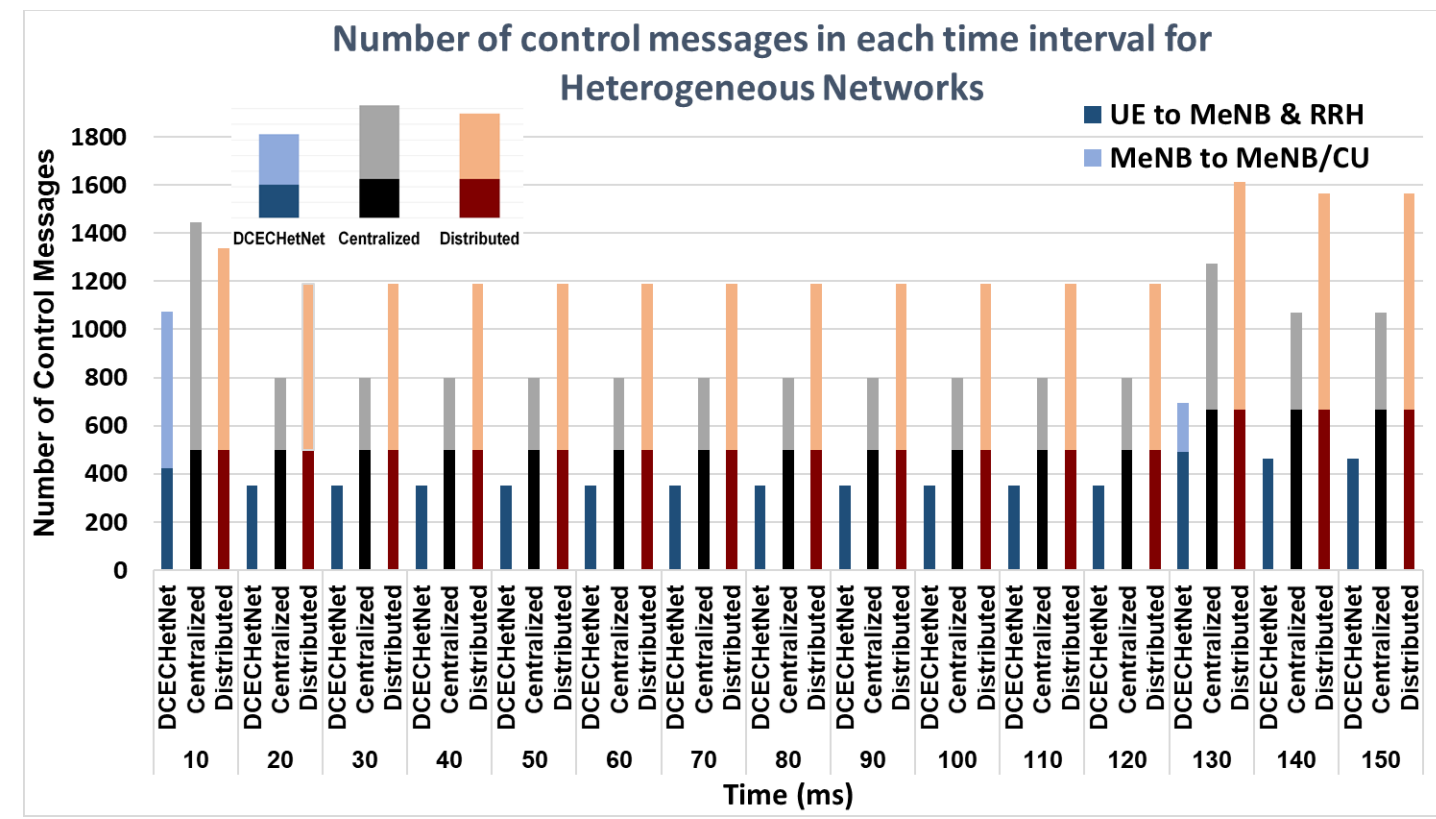

Figure 30: DCEC-HetNet, Centralized and Distributed based on the number of messages traveled in UEs to BSs and the Backhaul. Each three bars group represents 10ms (detailed view on the top left) 
For further evaluation of the DCEC-HetNet, we extended the simulation scenarios with 7 macro cells and 19 small cells, as shown in Figure 24(d), with a different density of active UEs. The UEs join and leave to the CoMP operation randomly. The CSI feedback periodicity is considered $5 \mathrm{~ms}$ and we use a longer simulation time. The results are collected and analyzed for the comparison of the number of control messages required for each of the three control architectures. Figure 31 demonstrates that by the use of DCEC-HetNet, the number of feedback messages can significantly be reduced in the network resulting in better throughput. This is because after an eNB is elected to act as the CS, the CSI feedback message does not need to exchange among the cooperating eNBs. This reduces the signaling load and the possibility of outdated CSI messages on the heterogeneous networks that eventually will improve the system performance. 
(a) Density of active UEs in CoMP $=6 / \mathrm{km}^{2}$

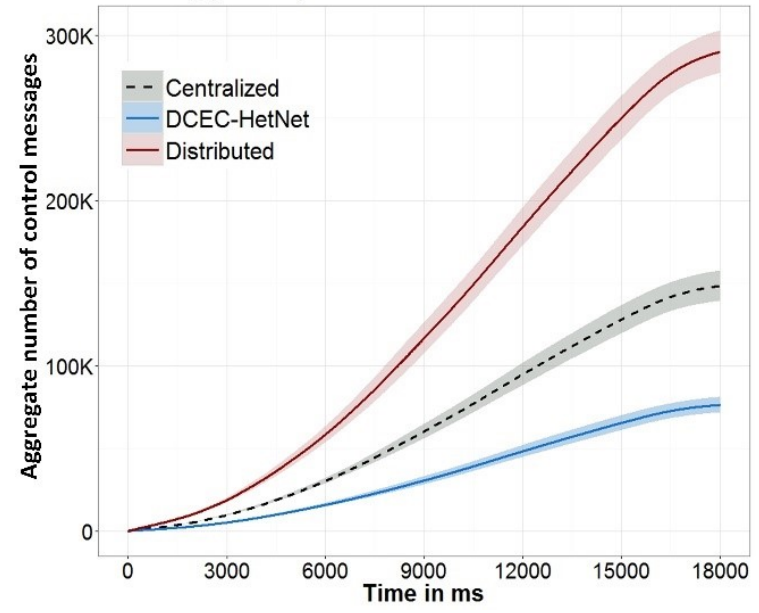

(c) Density of active UEs in CoMP $=17 / \mathrm{km}^{2}$

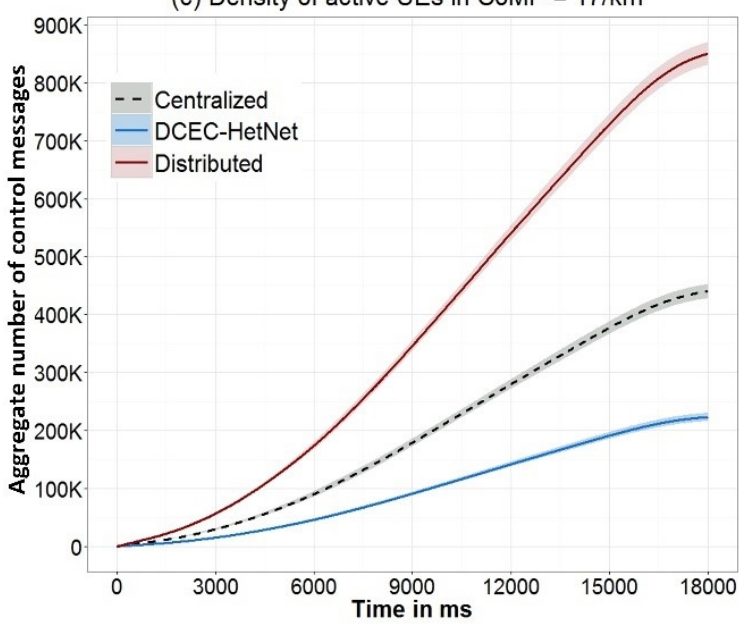

(b) Density of active UEs in CoMP $=11.5 / \mathrm{km}^{2}$

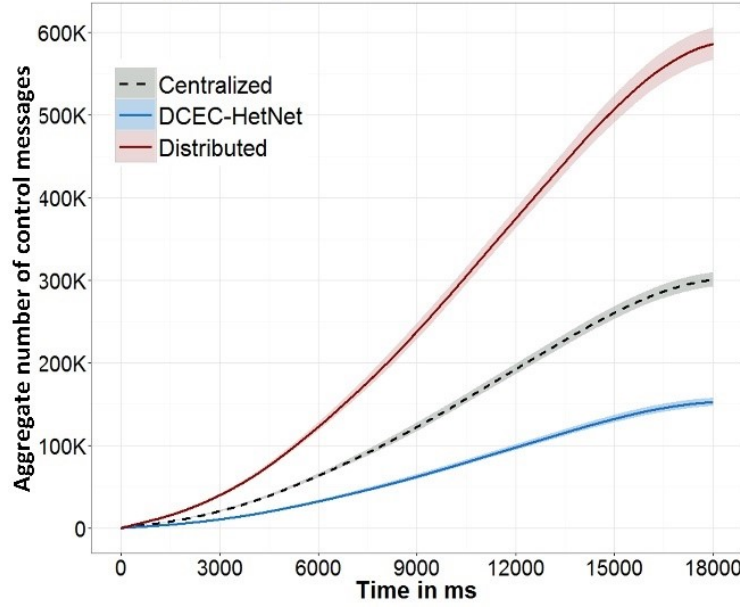

(d) Density of active UEs in CoMP $=23 / \mathrm{km}^{2}$

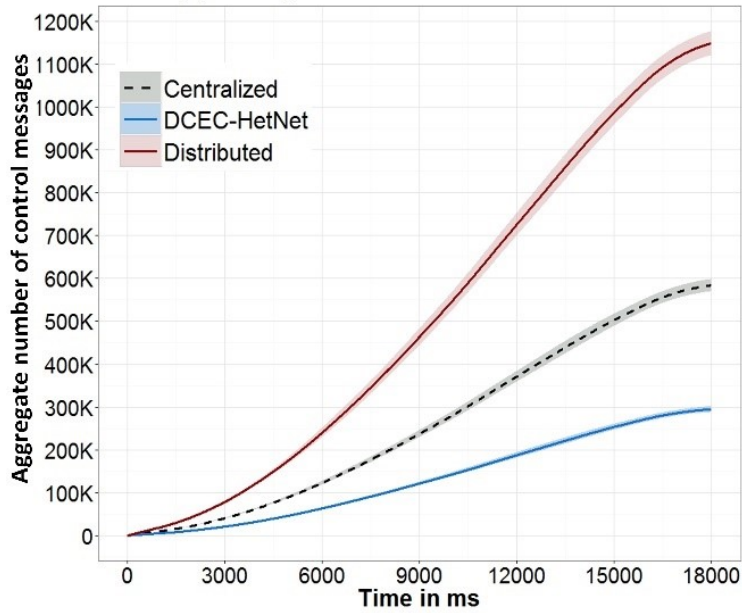

Figure 31: Aggregate number of control messages for Centralized, DCEC-HetNet and

Distributed based on the density of the active UEs into CoMP cooperation in heterogeneous networks

We increase the density of the networks in heterogeneous simulation scenarios including 7 macrocells and 70 picocells (10 picocells in each macro cell) as shown in Figure 24(e) and different density $\left(6 / \mathrm{km}^{2}, 11.5 / \mathrm{km}^{2}\right.$ and $\left.17 / \mathrm{km}^{2}\right)$ of active UEs in the CoMP operation to observe how the DCEC-HetNet works in dense heterogeneous networks. The CSI feedback periodicity is considered $5 \mathrm{~ms}$ and we use simulation time of 30 minutes. The UEs 
join and leave the CoMP operation randomly in the simulation time. Figure 32 demonstrates that by employing the DCEC-HetNet, the number of feedback messages significantly reduced. This once again confirm that DCEC-HetNet reduces the signaling load on the cooperative cellular networks.

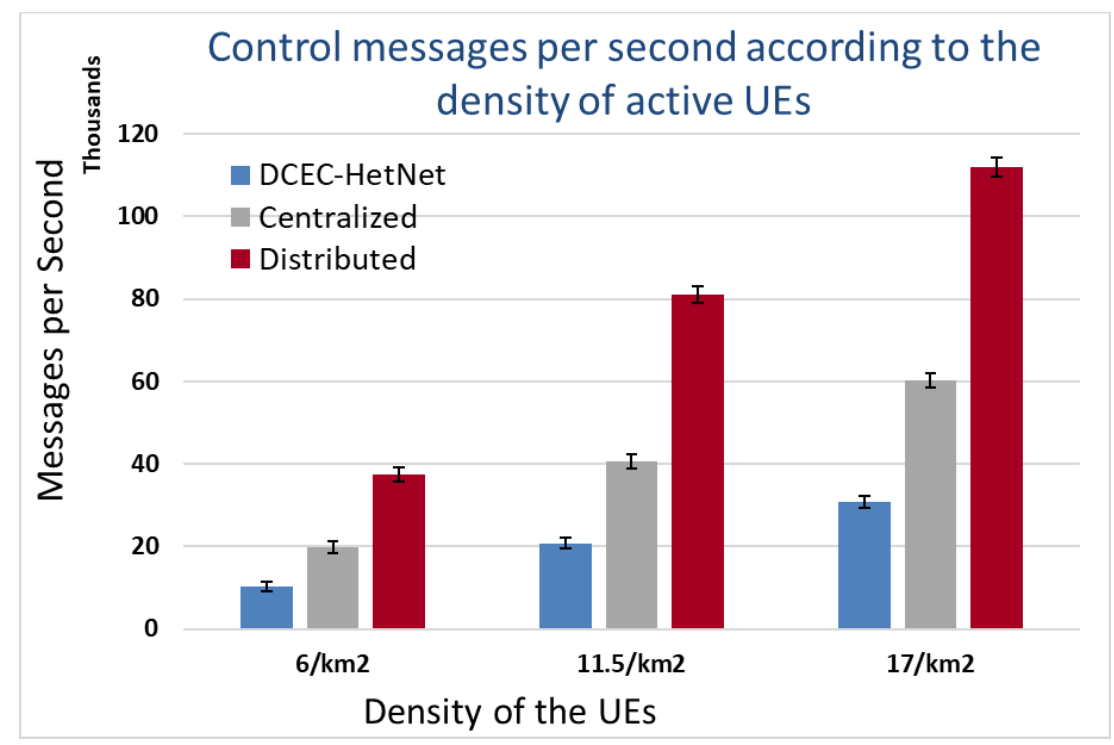

Figure 32: Number of control messages per second for DCEC-HetNet, Centralized and Distributed CoMP coordination architecture based on the density of the active UEs into in heterogeneous networks

According to the simulation results, shown in Figures 25 to Figure 32 we can see that DCEC coordination architecture has the potential to reduce the signaling overhead and feedback latency into both homogeneous and heterogeneous networks without changing the periodicity of the CSI feedback.

For further study, in Figure 33(a) we show the total amount of overhead in GB for 100 UEs in 30 minutes simulation time with respect to different CSI feedback schemes suggested by 3 GPP and other recent research works. In this case, we used the scenario as shown in 
Figure 24(e) and considered 100 UEs served in CoMP operation into the entire networks. For calculating the overhead, we used the equations (9-12) derived in Section 3.3 for four different CSI feedback schemes (wideband, subband level, UE selected best-M and full feedback). In every scheme DCEC-HetNet reduces the overhead significantly. In Figure 33(b), we present the signaling overhead per second with respect to different feedback schemes. Figure 33 clearly shows DCEC-HetNet also reduce the signaling overhead significantly with respect to the number of bits in every scheme of the CSI feedback that eventually will save the system bandwidth. This once again prove the improvement of the DCEC coordination architecture on top of the two other coordination architectures.

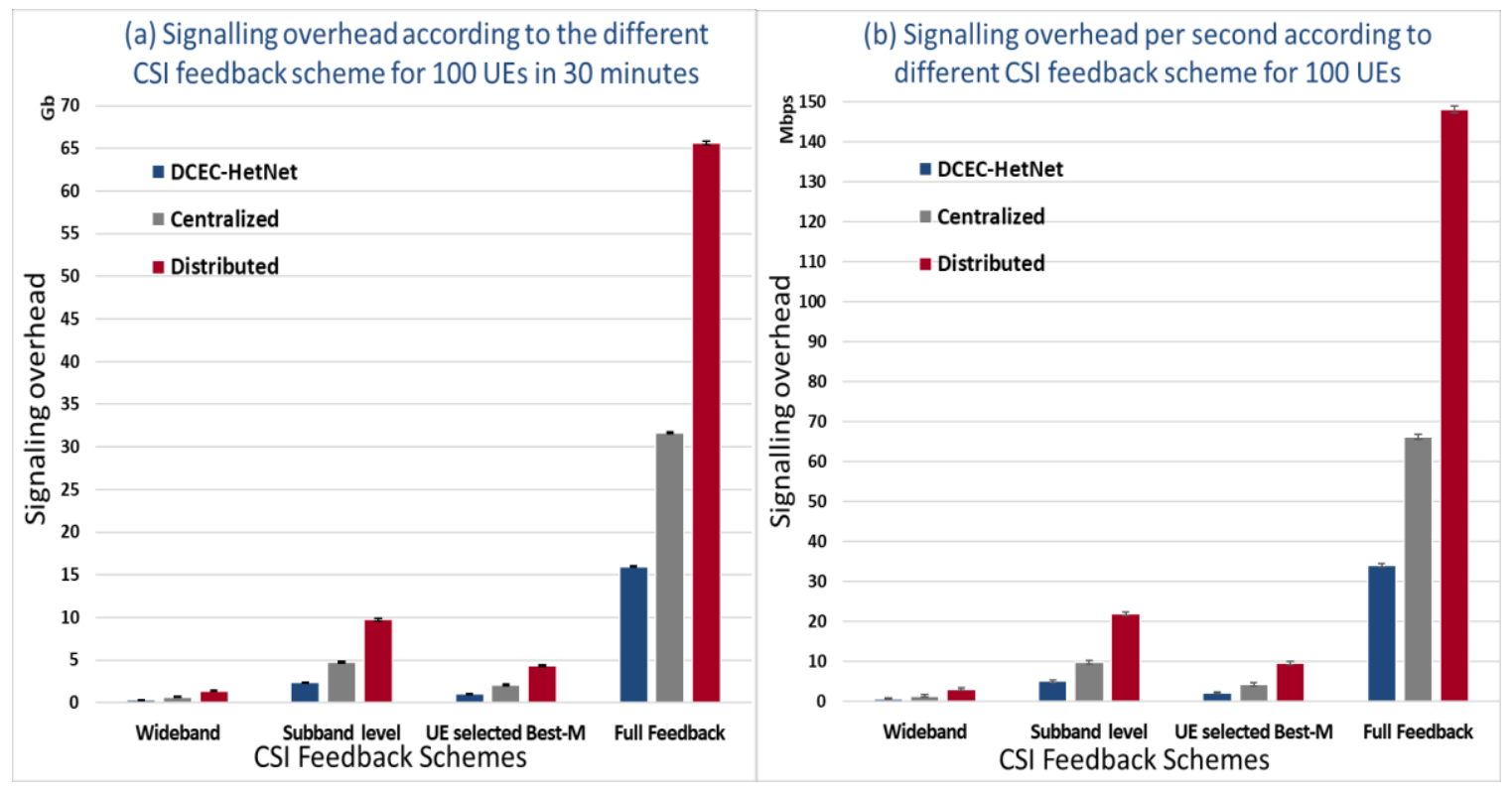

Figure 33: Signaling overhead in DCEC-HetNet, Centralized and Distributed CoMP coordination architecture with respect to different CSI feedback schemes for 100 UEs

The increase in CSI feedback messages might result in higher system delay on top of X2 latency. The feedback delay is measured for every CSI feedback sent by the UE and received by the scheduler. To find the average feedback delay of the system, we calculated 
the average of all the measurements for 30 separate simulation runs to minimize anomalies. Figure 34 shows the average CSI feedback delay of the entire system for a different number of UEs in the CoMP cooperation. In this case we consider a homogeneous network.

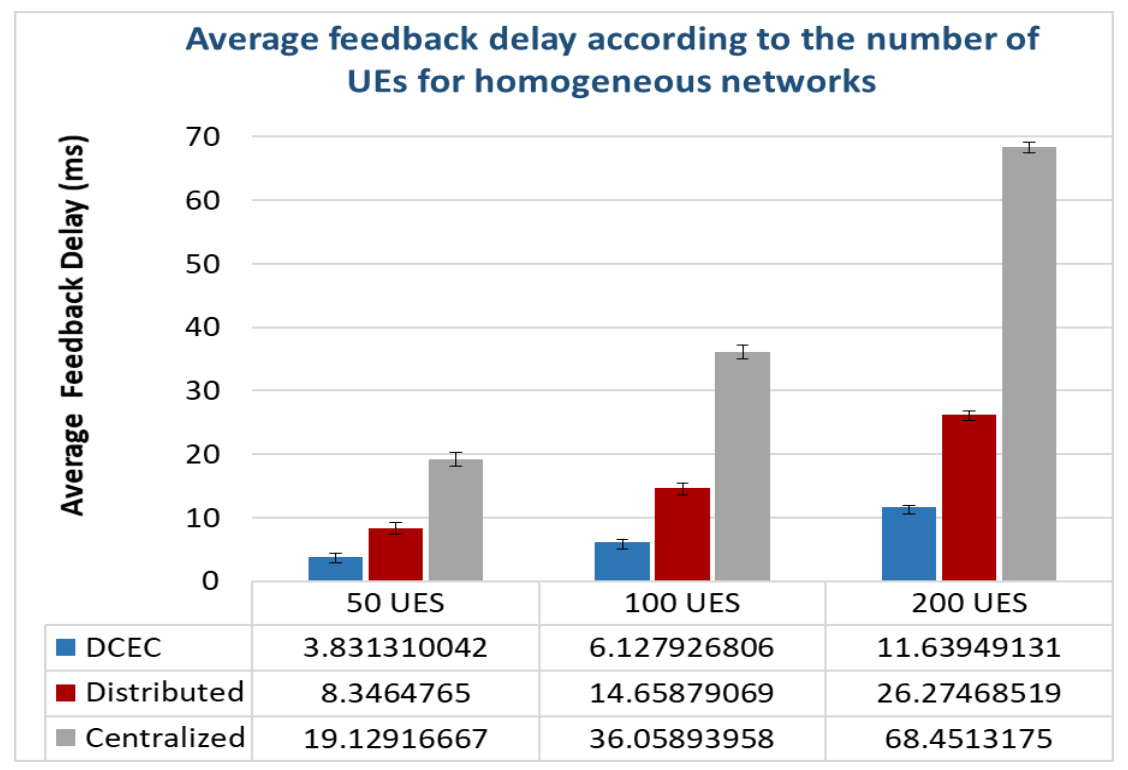

Figure 34: Average feedback delay for 50, 100, and 200 UEs in the network

The above figure shows that DCEC imposes the least amount of delay on the network while the centralized approach imposes the most system delay. It can be confirmed once again that the DCEC approach is less sensitive to the increase in the number of UEs and the number of cooperation set in the network. This allows for DCEC to be a good fit for both crowded and uncrowded areas. The reduction of the CSI feedback overhead and latency eventually improve the network throughput $[121,122]$.

\subsection{Summary}

The main goal of the CoMP approach is to improve the throughput of the network, especially for the cell edge users. However, the two standard architectures of CoMP (centralized and distributed) face some challenges such as latency, signaling overhead and 
infrastructural overhead. The promising gain of CoMP largely depends on these overhead and latency. In this chapter, we presented a novel coordination architecture named DCEC for CoMP operation in homogeneous cellular networks to reduce the latency and the signaling overhead so that the overall performance of the network could be improved. The architecture also extended for heterogeneous networks named DCEC-HetNet. The simulation results show that the DCEC and DCEC-HetNet coordination architecture for CoMP reduce the signaling overhead and the CSI feedback latency compared to two other standard CoMP coordination approaches which eventually will improve the network performance. Given that DCEC does not need any additional hardware for implementation, switching to DCEC could decrease the signaling load and feedback latency, and increase the system throughput at minimal cost. 


\section{Chapter: Handover Enhancement for Dense Heterogeneous}

\section{Cellular Networks}

Network densification, such as ultra-dense heterogeneous network (UDHetNet) is considered as a key enabler to achieve the goals of $5 \mathrm{G}$ cellular networks. World leading wireless system design and device manufacturing industries publicly stated that dense small cells are the foundation to achieve $1000 \times$ capacity challenge in the $5 \mathrm{G}$ wireless cellular networks $[123,5]$. UDHetNets consist of macro-cells coexisting with dense low power cells such as pico-cells, femtocells and remote radio head (RRH). We discussed details about the UDHetNet in Chapter 2. These small cells use lower transmit power, hence provide a small coverage area, and they can significantly improve the network capacity by spectrum reuse and improving the link efficiency by reducing the distance between the access nodes and the users. Figure 35(a) shows the overall architecture of ultra-dense heterogeneous cellular networks.

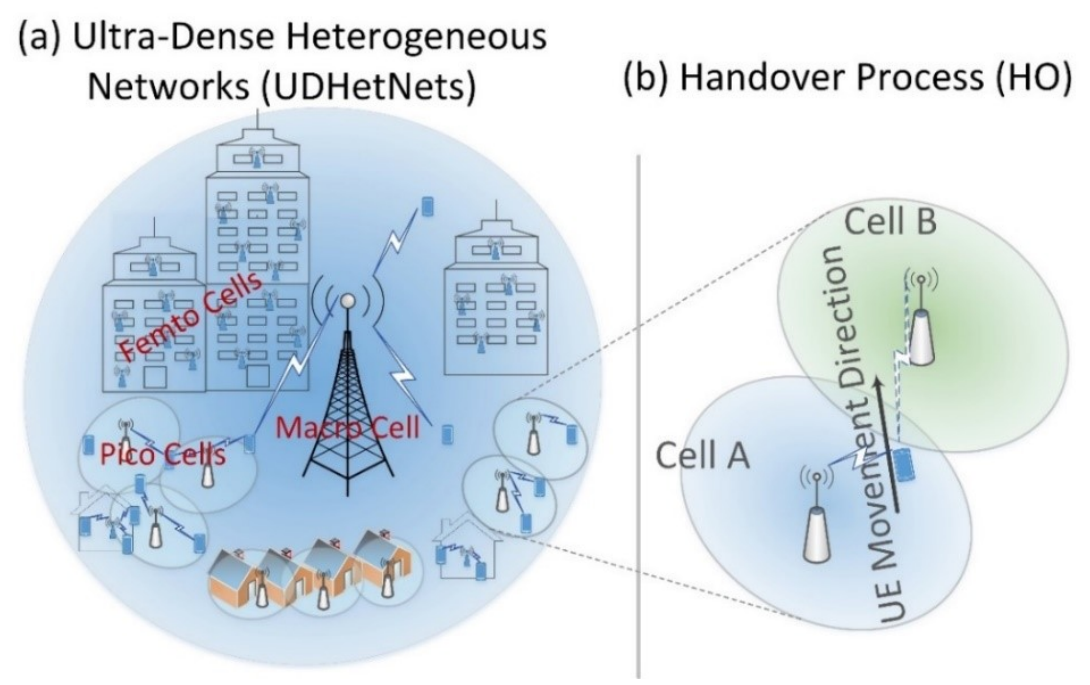

Figure 35: A simplified view of ultra-dense heterogeneous networks and handover 
However, these networks face new technical challenges such as mobility management and intercell interference as we stated in Chapter 2. Since the coverage area of a cell is small, users' equipment (UEs) experience frequent handover (HO), and handover oscillation. The 3rd generation partnership project (3GPP), telecommunications standardization body showed that the increase in the number of handovers in small cell networks compared to macro-only networks can be up to $120 \%-140 \%$, depending on the UE speed [9]. In UDHetNets, the number of handovers could be even higher, depending on the UE speed and density of the cells. Figure 36 provides an overall idea of densification and its impact on the networks. Therefore, to realize the potential link efficiency and capacity benefits of dense small cells, we need adequate mobility management, and this has become a major technical challenge in the UDHetNets. In this chapter we present a novel handover approach named Enhanced Handover for Low and Moderate speed UEs (EHoLM) that reduce the number of handovers and handover oscillations in dense heterogeneous cellular networks.

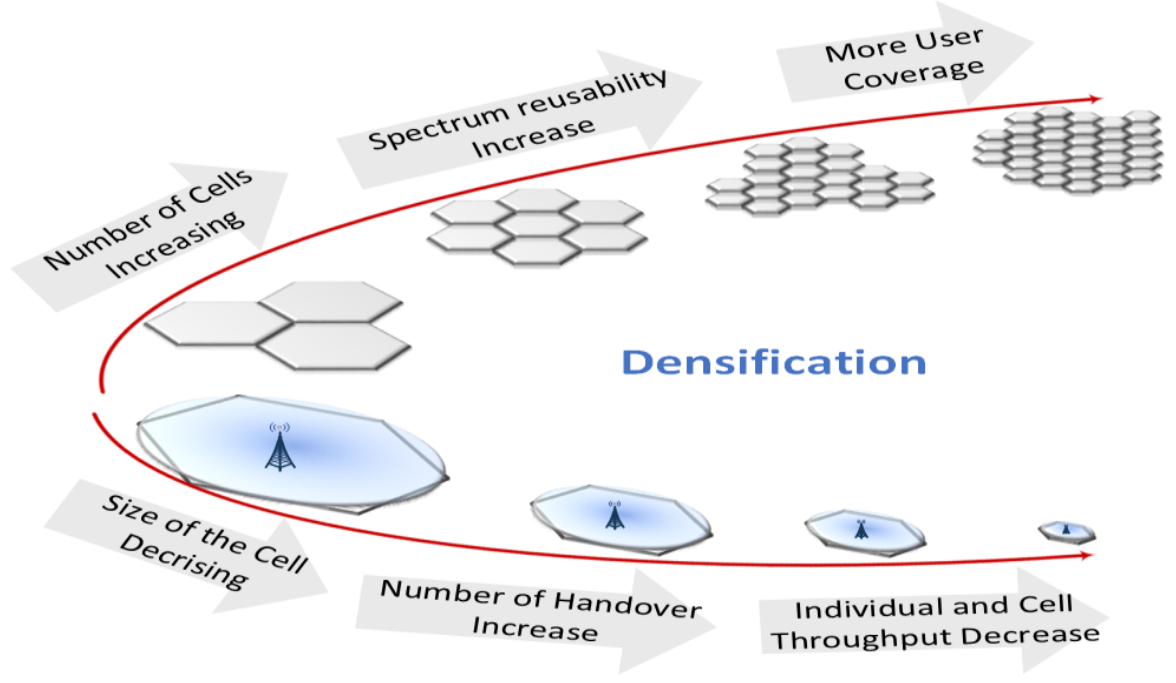

Figure 36: Densification and its impact in the networks 
The handover process is used to support the seamless mobility of a UEs. The HO process makes UEs in active mode to be transferred from the serving cell to the neighboring cell with the strongest received power, and the user is not aware, as shown in Figure 35(b). In conventional homogeneous cellular networks, typically same set of handover parameters are used in all over the networks. However, in HetNets, if the same set of parameters is used for all UEs and all the types of cells, there is a possibility to degrade the mobility performance [124]. The increase in the number of handovers will increase the control overhead and the switching load into the network that will eventually decrease the network performance. Maintaining low handover failure (HOF) rate is also important for better user experience. Therefore, it is essential to analyze the handover parameters and to enhance them for heterogeneous cellular networks. In the next subsection we discussed the handover procedure for LTE and LTE-Advanced cellular networks.

\subsection{Handover Process in Wireless Cellular Networks}

3GPP specifies a handover procedure and mechanism for LTE and LTE-Advanced mobile networks that support user's mobility. In LTE-advanced cellular networks, UE-assisted network-controlled handovers are performed [125]. In UE-assisted network-controlled handovers, the serving eNB makes the decision to move from one cell to another based on the measurement report (MR) received from the UE. The handover procedure of $3 \mathrm{GPP}$ LTE and LTE-A is defined in [125, 32]. A HO process, in general completes in five steps.

1. A UE measures the downlink signal strength periodically.

2. It processes the measurement results.

3. UE sends a measurement report (MR) to the serving eNB based on predefined $\mathrm{HO}$ 
criteria.

4. The serving eNB takes the handover decision based on the received MR.

5. Finally, the UE receives the handover command from the serving eNB and completes the handover process.

For modeling, the $\mathrm{HO}$ processing of a UE is also divided into 3 states [32]:

- State 1: Before the handover criteria (A3 event) is satisfied.

- State 2: After the handover criteria is satisfied but before the handover command is successfully received by the UE.

- State 3: After the HO command is received by the UE, but before the HO process is completed successfully.

Figure 37 shows the different states of handover process and corresponding message sequences $[125,32,126]$. 


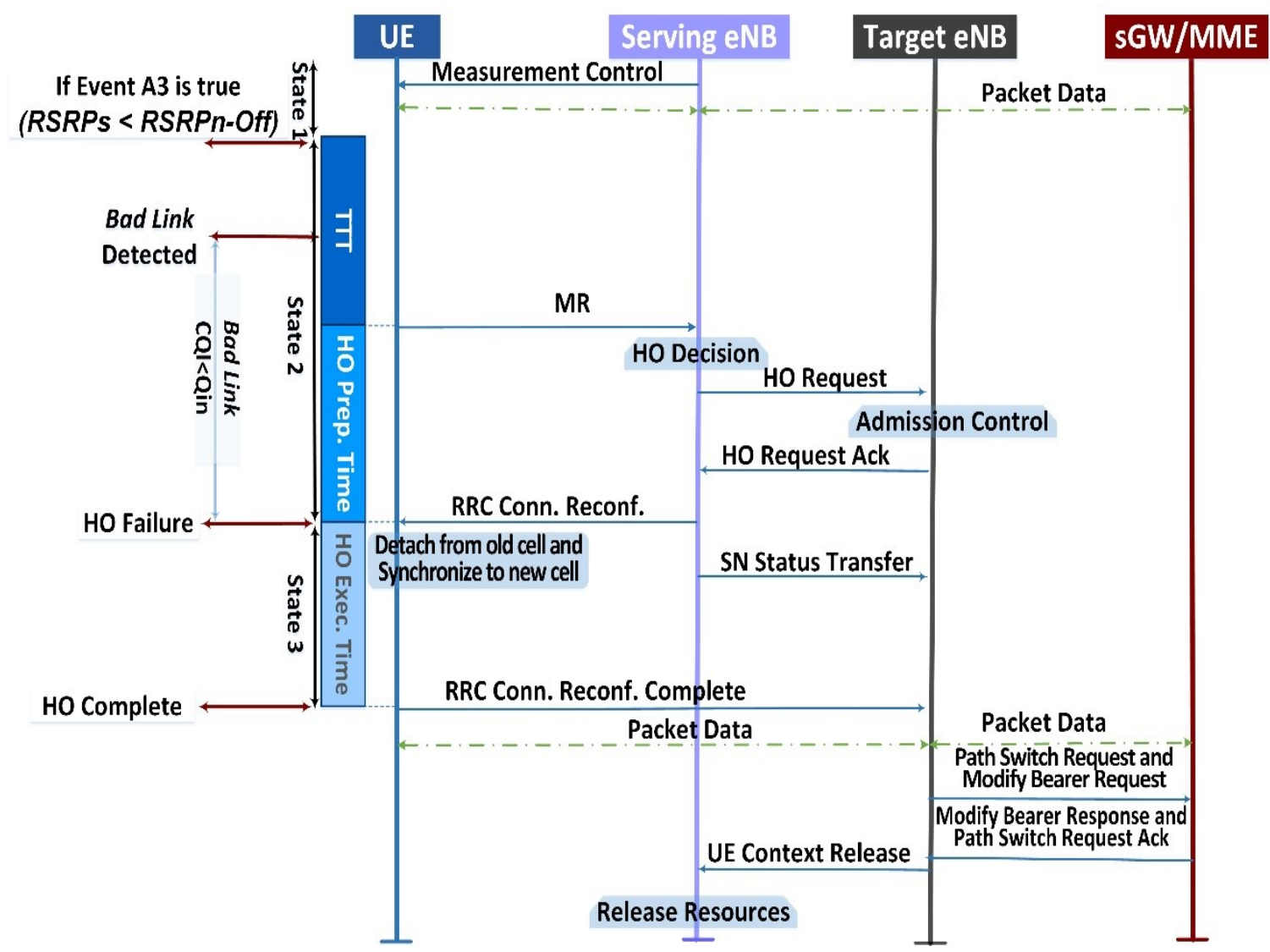

Figure 37: Simplified handover process for LTE and LTE-A cellular networks

The UE calculates reference signal received power (RSRP) every 40ms and performs a linear average over 5 successive RSRP samples based on the following formula [127, 128, 124].

$$
M(n)=\frac{1}{5} \sum_{k=0}^{4} R S R P_{l 1}(5 n-k), \quad \text { Equation } 16
$$

where,

$R S R P_{l 1}:$ RSRP sample measured every $40 \mathrm{~ms}$

n: discrete time index of the RSRP sample

$\mathrm{k}$ : delay index of the filter 
Therefore, the handover measurement period for a UE in layer three (L3) is 200ms. Once the L3 filtered RSRP of the target cell is higher than the RSRP of serving cell plus A3 offset or hysteresis margin, the UE starts TTT, the Time to Trigger Timer [124, 129].

$$
\text { Event A3: RSRP } s+\text { Offset }<\operatorname{RSRP}_{\mathrm{n}}
$$

Equation 17

where, $\mathrm{RSRP}_{\mathrm{s}}$ is the RSRP of the serving eNB and RSRP $\mathrm{n}$ is the RSRP of a neighboring eNB. The handover process is performed mainly via the radio resource control (RRC) layer between UE and eNB in the control-plane. The simplified message sequence diagram of LTE and LTE-Advanced handover process is shown in Figure 37 [27]. If the A3 event condition as shown in equation 17 is true throughout the TTT, the UE sends measurement report (MR) to the serving eNB once TTT expires. This MR kicks off the handover preparation phase. The serving eNB issues a handover request message to the target cell. This handover request carries out admission control procedure for the UE in the target cell. After completing the admission control, target eNB sends a handover request Ack message to the serving eNB. When the serving eNB receives the handover request Ack, data forwarding from serving eNB to target eNB starts and the serving eNB sends a handover command (RRC Conn. Reconf) to the UE. UE then synchronizes with the target eNB and sends a handover complete message to the target eNB. As a result, intra eNB handover process of the UE is complete, and the target eNB becomes its serving eNB and starts transmitting data to the UE. The new serving eNB sends a path switch request to the serving gateway to inform the core network that it is the new serving eNB for the UE. The serving gateway or the network sends a modify bearer response message to the new serving eNB and switched the downlink data path from previous serving eNB to new serving eNB. Finally, new serving eNB sends a message to the old serving eNB requesting to release the 
resource for the UE.

\subsection{Enhanced Handover for Low and Moderate Speed UEs}

Despite the promising features of UDHetNets, they have introduced new challenge on mobility management and interference coordination as we mentioned earlier. The handover performance largely depends on the handover parameters such as Time to Trigger (TTT) and A3 offset $[128,124]$. On the other hand, CoMP improves the performance of cell edge users by reducing the interference and serving the UE jointly [14, 27]. As a result, in CoMP cooperation, UEs receives better signal quality than in a conventional transmission though it is in the cell edge. The performance of CoMP also depends on the CoMP threshold. The handover and CoMP both happen on the UEs in the cell edge region and both have their own parameters. Therefore, to achieve the better system performance we need to optimize the handover parameters when the UE served in CoMP cooperation in UDHetNet.

In CoMP enabled networks, if a conventional handover process is used, some handover might occur though the UE is still in CoMP transmission and served by the same CoMP cooperating set. That is, the serving eNB still serves the UE with other cooperating eNBs, but the UE is handed over to another eNB in the CoMP set. This is an unnecessary handover, which eventually degrades the system performance. Considering this, the EHoLM algorithm exploits CoMP and the dual connectivity provided for control plane and user plane separation for UEs. In this approach, the handover criteria will not be satisfied until a UE moves from a CoMP to no-CoMP region in a different eNB, or a UE moves from one CoMP cooperation set to a different CoMP set without the current serving eNB (instead of conventional handover criteria A3 event as shown in equation 17). 


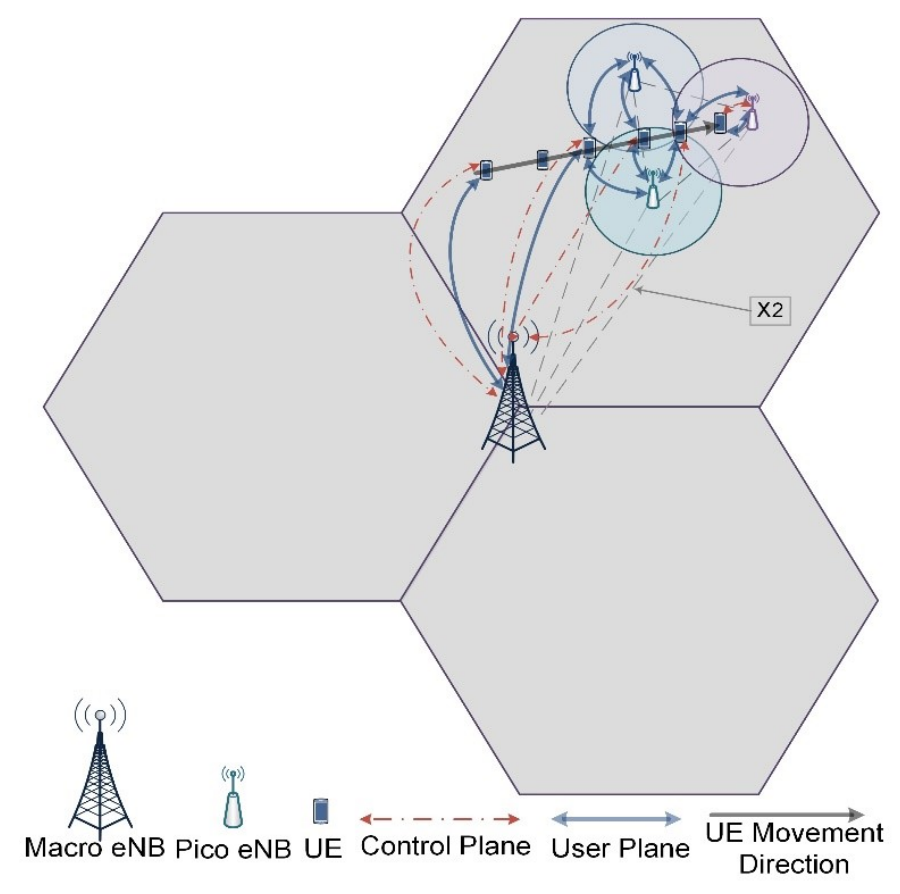

Figure 38: Simplified view of the EHoLM handover scheme

Now, consider a UE is moving gradually from its serving eNB to the target eNB, and consider that CoMP has been established by more than one eNB (including the serving and target eNBs) to serve the UE. If the $\mathrm{A} 3$ offset ( $0 \mathrm{~dB}$ to $3 \mathrm{~dB})$ [32] in the handover is less than the CoMP threshold $(3 \mathrm{~dB}$ to $9 \mathrm{~dB})[68,26]$, there are some handovers happen, although the UE is still in the CoMP transmission with same cooperating set. That is, the UE is handed over to another eNB, but it is still served by all the eNBs together. This is an avoidable handover, which degrades the performance of the networks. We want to take the advantage of CoMP, which provides a better signal strength to the cell edge UEs by reducing the ICI as well as dual connectivity that provides control plane and data plane separation for UEs. In EHoLM approach, the handover criteria will not be satisfied until a UE moves from a CoMP operation to non-CoMP operation of a different eNB or a UE 
moves from one CoMP cooperation set to another CoMP cooperation set that does not contains the current serving eNB instead of the conventional handover criteria discussed in equation 17. That is, if a UE moves from a macro cell to a CoMP region, it will stay connected to the macro eNB (serving eNB) until it leaves CoMP and moves to a no CoMP region of another eNB. A simplified diagram of EHoLM scheme is shown in Figure 38. In this figure, dashed lines represent the control plane connectivity and solid lines represent the user plane connectivity. Initially, when the UE is in the no CoMP region of the macro eNB, it is connected to the macro eNB both control and user planes. Gradually, when the UE moves to the CoMP region, it is served by more than one eNBs in user plane, but it remains connected to the serving eNB in the control plane. Finally, when it moves from the CoMP to the no CoMP region of the pico eNB, it is handed over to the pico eNB. For better understanding we present a flowchart of the EHoLM scheme in Figure 39. 


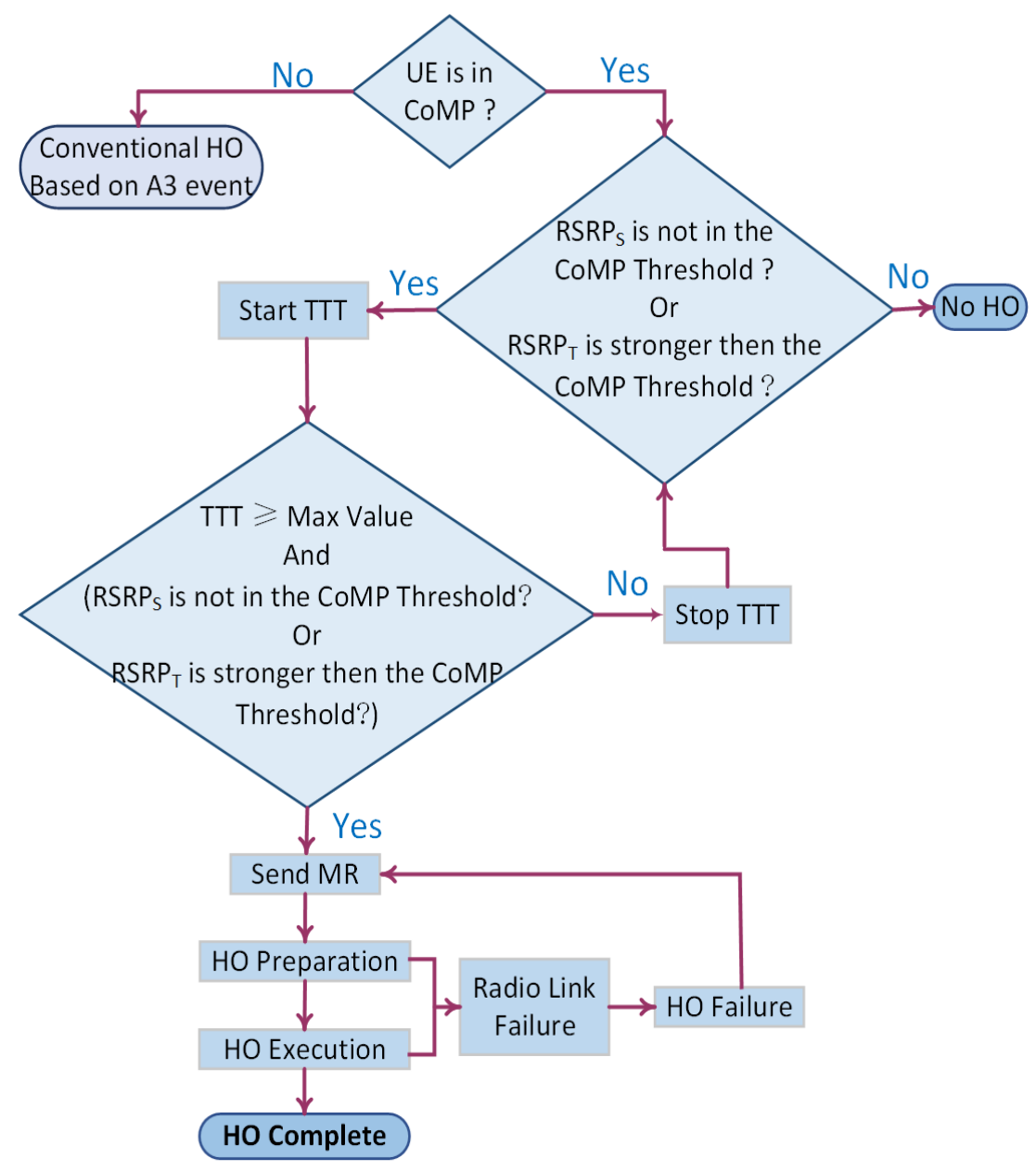

Figure 39: EHoLM handover scheme flowchart

\subsection{Modeling Handover and Oscillation in cellular networks}

The handover is a process that consumes radio resources, which are limited; therefore, it is important to minimize the number of handovers and handover oscillations. In this section we present how we modeled handover oscillation and how the number of handovers and handover oscillations are counted in the simulation. If a UE handed over from cell $u$ to cell $v$ and then another handover back from cell $v$ to cell $u$ within the minimum time-of-stay (MTS) we have a handover oscillation. The time-of-stay (TS) in cell $v$ is the duration of time from when the UE successfully complete the handover to cell $v$ to when the UE 
successfully sends the handover complete message to cell $u$. We considered MTS $\leq 1$ second for simulation $[129,32]$. Moreover, if a UE stays in a cell is less than the MTS, the handover also is considered as unnecessary [32]. Therefore, reducing the number of handovers and handover oscillations are the two important metrics in handover performance evolution. Figure 40 shows how we modeled the handover oscillation in the left and how we count their number of handovers and handover oscillations in the right. To count the number of handovers and handover oscillations, we follow the 3GPP specification as discussed in $[130,9,32]$.
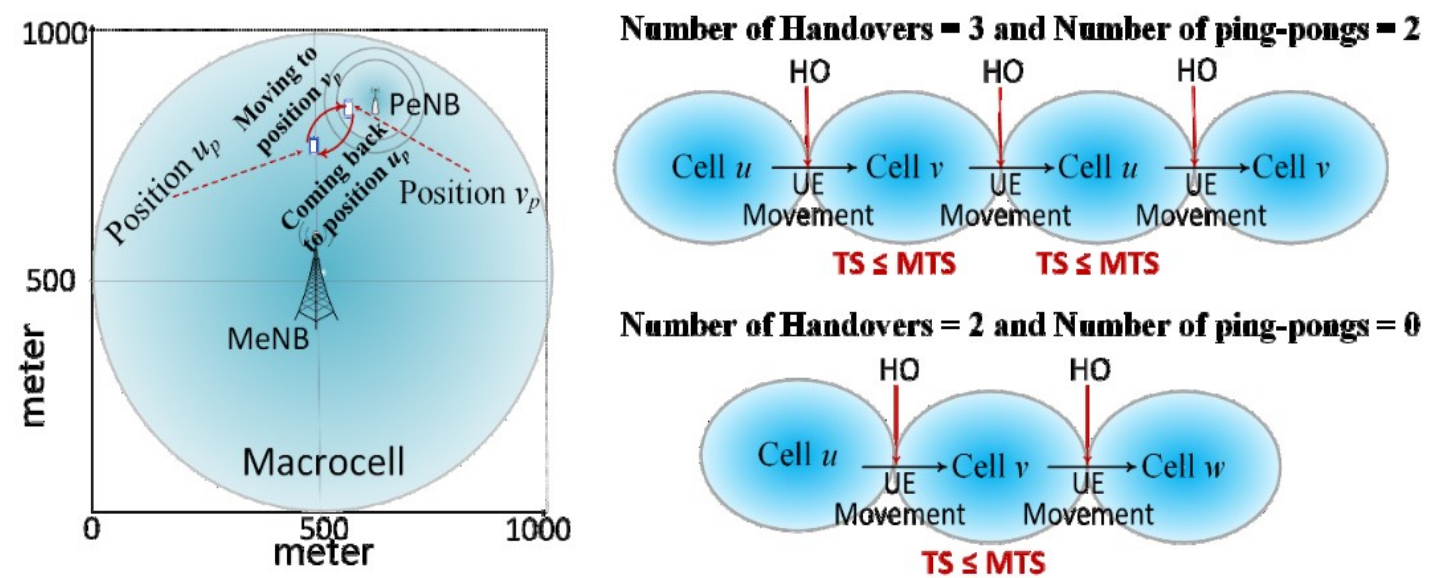

Number of Handovers $=2$ and Number of ping-pongs $=0$

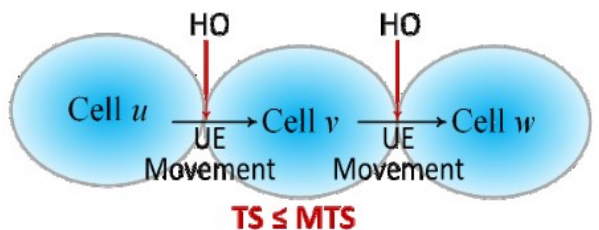

Figure 40: Handover oscillation modeling and computing the number of handovers and handover oscillations

In this chapter, we focus on how to reduce the number of handovers and handover oscillations in the UDHetNets to improve the system performance. In the simulation scenarios, the minimum distance between macro eNB (MeNB) and pico eNB (PeNB) is considered 100 meters and the minimum distance between PeNB and PeNB is considered 50 meters. In case of handover, UEs are randomly placed all over the coverage area of the 
network and move straight in a random direction to its destination. For handover oscillation UEs are randomly placed closer to the border of macrocells and picocells (point $u_{p}$ ). The UEs then move straight in a random direction with an angle to the point $v_{p}$ of cell $v$, which is also closer to the border of the cells. The UEs move back and forth continuously between an initial position and final position until the simulation ends

\subsection{Modeling the EHoLM in HetNets using DEVS}

To study the handover procedure with decoupling the control plane and user plane and CoMP we consider heterogeneous networks as suggested by 3 GPP in $[9,32]$. We designed a DEVS model to examine the performance of the EHoLM handover procedure in dense heterogeneous cellular networks. The simplified structure of the model is shown in Figure 41. The top level coupled model is the geographic area, which includes a number of macro and small cells. Each macro cell contains one MeNB, multiple PeNBs and many UEs. The numbers of PeNBs and UEs vary based on different scenarios. Each MeNB, PeNB and UE coupled model is composed of two atomic models named Buff and Proc. In this model we reuse the Buff atomic model from DCEC model as stated in Chapter 3. This is (reusability) one of the advantages of DEVS formalism. The UEProc calculates the RSRP based on the formula we discussed in equation 16. According to the handover criteria, UEProc generates the MR, starts the TTT and sends it to the MeNB or PeNB through the output port (Out), when TTT reached the maximum value. The MeNB and PeNB stores that in their own Buff. The MeNB Buff and PeNB Buff acts as a buffer for the MeNB or PeNB coupled model. Once the MeNB or PeNB receives message, the MeNB Buff or PeNB Buff pushes it in a queue as we mentioned before. The message is popped out from the queue and forwarded to the MeNBProc or PeNBProc when a request is received from the processor. The 
MeNBProc takes the $\mathrm{HO}$ decision based on the MR it received from the UE and sends the $\mathrm{HO}$ request to the target eNB through the output port (X2Out).

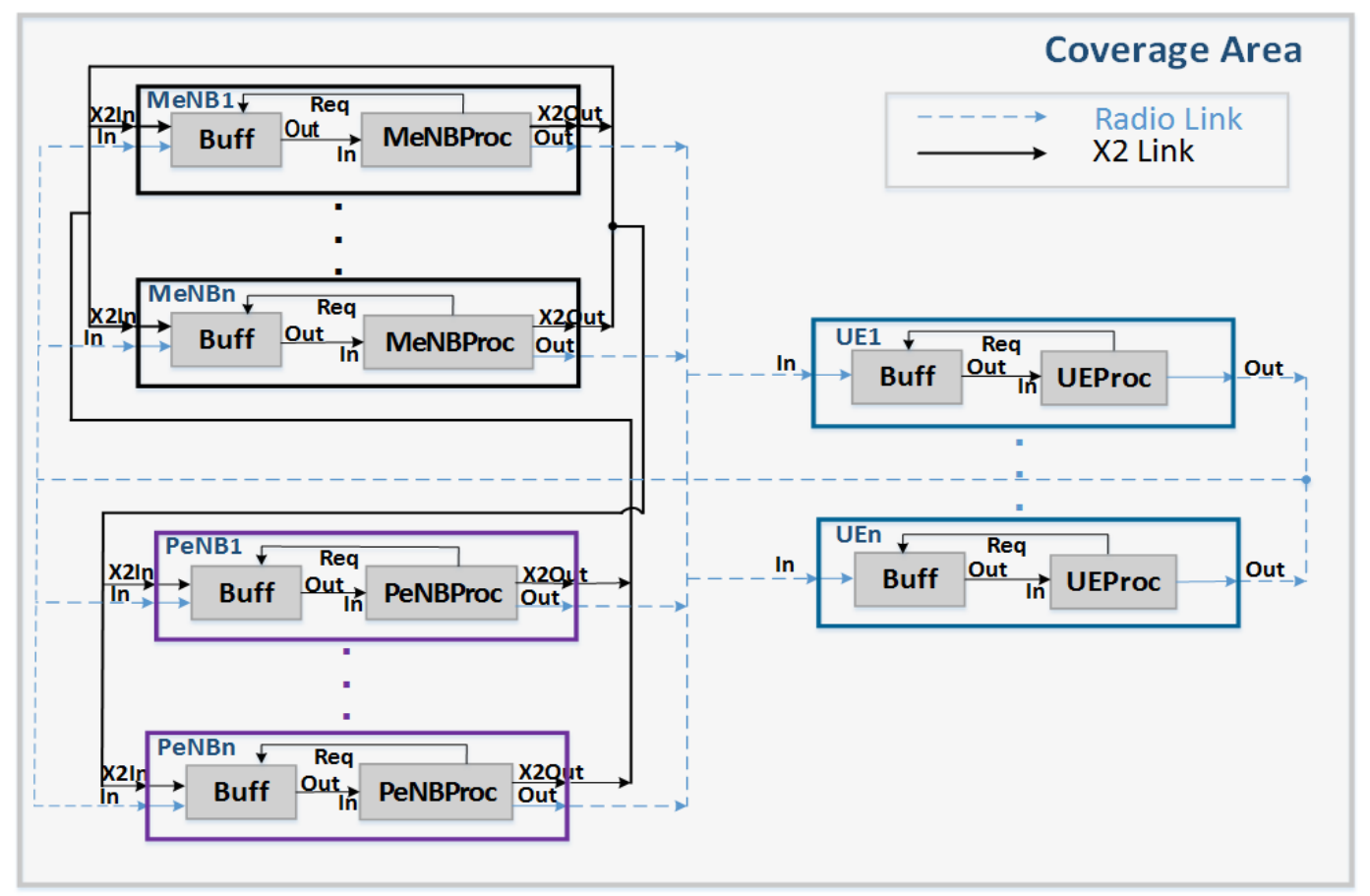

Figure 41: Simplified DEVS model for EHoLM in heterogeneous networks

In the Figure 41, the black solid links represents the X2 connections between eNBs (MeNBs and PeNBs). The blue dotted lines show the radio links between the MeNBs to UEs and PeNBs to UEs. Moreover, the number of MeNBs, PeNBs and UEs could be any number according to the simulation scenarios. Finally, all the MeNBs, PeNBs and UEs together composed the top-level coupled model, which is the network coverage area. The model is implemented in $\mathrm{CD}++$ toolkit and presented in the next section.

\subsubsection{Model Implementation}

The model is implemented in the $\mathrm{CD}++$ toolkit, an open source simulation platform that implements DEVS and Cell-DEVS methodology. Figure 42 below depicts a simplified 
UML class diagram of the model discussed above. The MeNBProc class and the PeNBProc class implement the MeNBProc atomic model and PeNBProc atomic model as shown in Figure 41 respectively. MeNBProc and PeNBProc classes characterize with id, position, transmit power, frequency, serving eNB, target eNB etc. The UEProc class characterizes with the properties such as id, position, speed, power, TTT, offset etc. The UEProc class, atomic component, calculates RSRP every 40ms and generate MR based on the formula discussed before. This atomic model checks the handover criteria, if it satisfies, start TTT. When the TTT reaches to the maximum assigned value it sends the MR to its serving MeNB or PeNB through the output port (Out). Movement manager keeps track of the UEs moving from current position to the destination position runtime. The globalBuffer class represents the buffer for each of the MeNB, PeNB and UE, which is the same as Buff we used in Chapter 3. That is, we are reusing the globalBuffer atomic component in this model, which is an advantage of DEVS because of its hierarchical nature. Msg class define all types of messages exchange in the networks. 


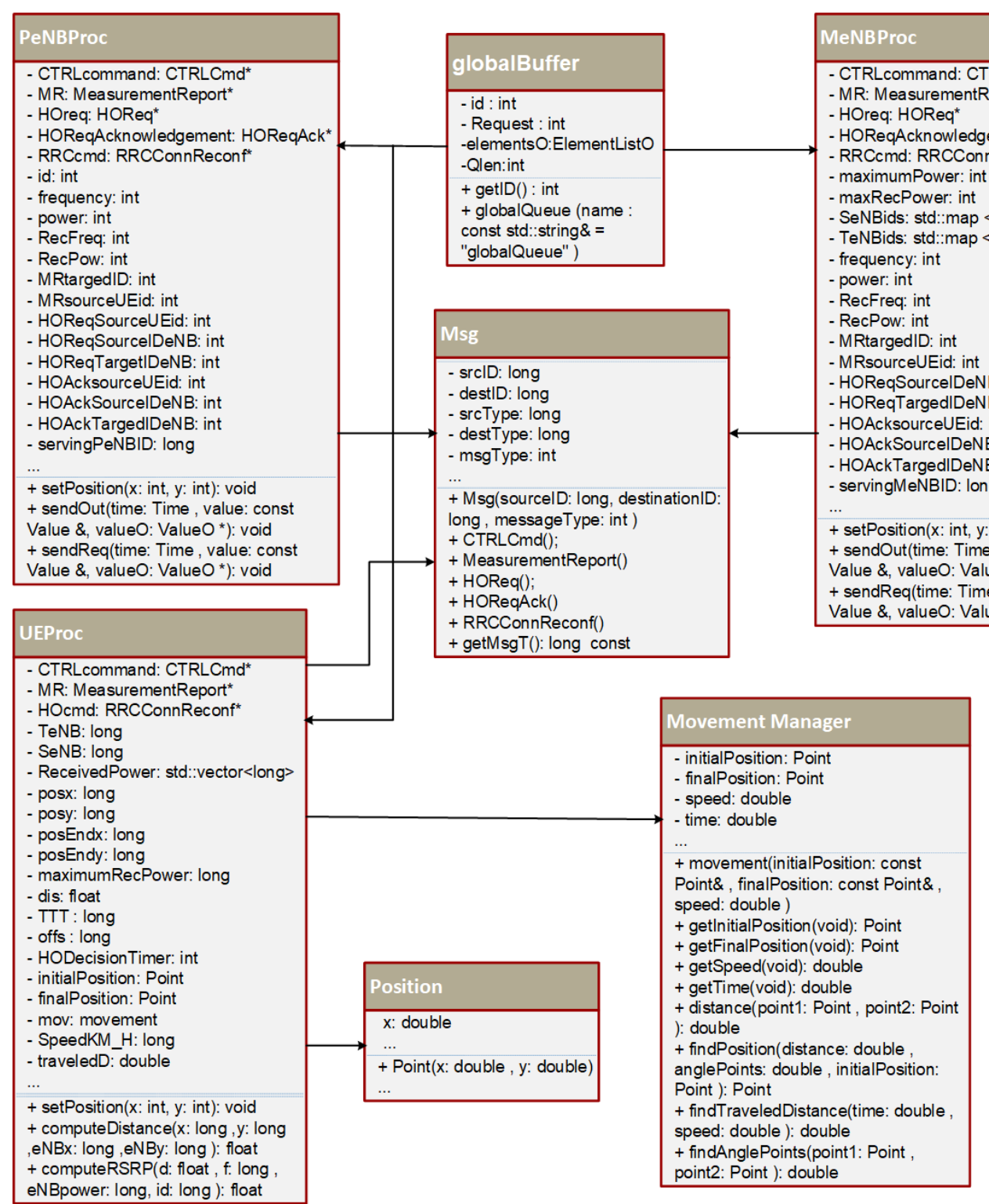

Figure 42: Simplified class diagram of the DEVS model for EHoLM handover scheme

Once the eNB receives a message, the eNB Buff pushes it in a queue. The message is popped out from the queue and forwarded to the eNB processor (eNBProc) to process when a request is received from its processor. The eNBProc takes the $\mathrm{HO}$ decision based on the received MR from the UE and sends the $\mathrm{HO}$ request to the target eNB through the output port (X2Out) as all the MeNBs and PeNBs are connected by X2 links. The number of MeNBs, PeNBs, and UEs could be different according to the simulation scenarios. 


\subsection{Simulation Scenarios and Results}

To study the EHoLM handover procedure in the context of dense HetNets, we considered the scenarios suggested in $[131,9,12,32]$. Figure 43 shows the simplified network architectures of the simulation scenarios we used. The network scenario in Figure 43(a) has 1 macro cell and 24 Pico cells separated by the minimum ISD as mentioned in Table 11. Figure 43(b) shows a dense HetNet with 7 macro cells and each macro cells has 16 small cells. Small cells are placed randomly following the minimum ISD mentioned in Table 11. Figure 43(c) shows a dense HetNet with 19 macro cells and 72 picocells. In this scenario placement of the PeNBs are in the middle of each of the six borders of hexagonal cells. The number of UEs varies in each network scenarios and discussed later in details. The UEs are considered initially connected to the eNBs with strongest received power and move in random directions over the simulation area.

We ran a series of simulations on both EHoLM and the conventional handover model, based on the initial conditions summarized in Table 11. These simulation parameters have been chosen based on the 3 GPP specifications and other related works $[132,68,133,9$, $114,111,27]$. We simulated EHoLM and the conventional handover process as mentioned in the previous section using different scenarios.

In our simulation scenarios, cells are considered macrocells and picocells in an urban area. The propagation model is considered, based on 3GPP standard in [134, 9] as follows: 
Pico Cell: $147+36.7 \log _{10}(\mathrm{~d})$,

Equation 19

where $d$ is the separation between UEs and eNBs.
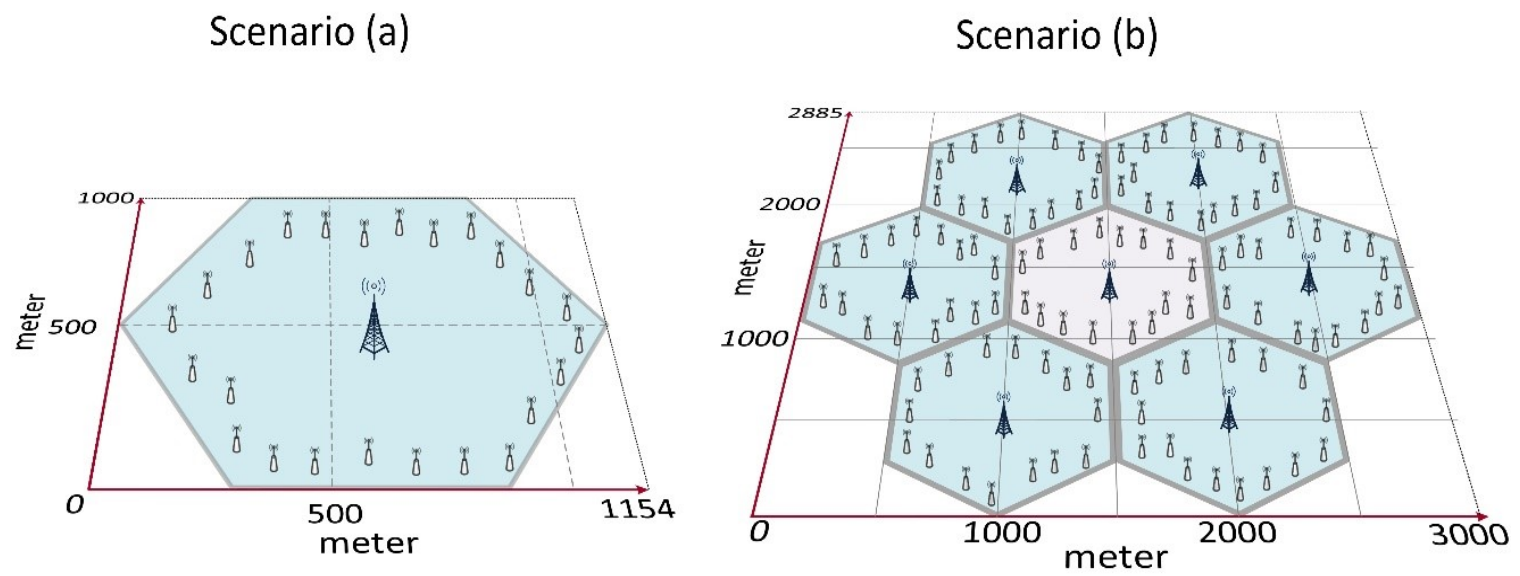

Scenario (c)
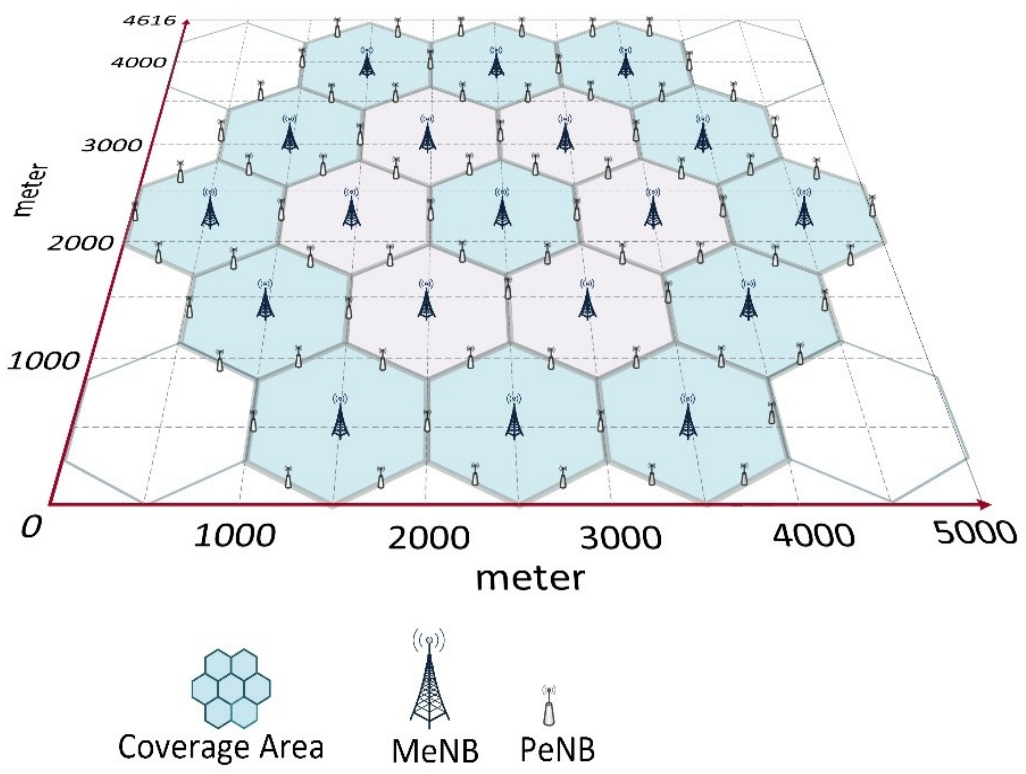

Figure 43: Simplified simulation scenarios with MeNB and PeNB placement 
Table 11: Initial assumptions for the simulation

\begin{tabular}{|c|c|c|}
\hline Parameters & Macrocell & Picocell \\
\hline Number of eNBs & 1,7 and 19 & 24,72 and 112 \\
\hline Transmit power & $43 \mathrm{dBm}$ & $30 \mathrm{dBm}$ \\
\hline Carrier Frequency & $2000 \mathrm{MHz}$ & $3500 \mathrm{MHz}$ \\
\hline Path loss model & $128.1+37.6 \log _{10}(d)$ & $147+36.7 \log _{10}(\mathrm{~d})$ \\
\hline Number of UEs & \multicolumn{2}{|c|}{$25,50,100,200$} \\
\hline UE Distribution & \multicolumn{2}{|c|}{$\begin{array}{l}\text { Uniform: randomly into the simulation area } \\
\text { Uniform: randomly into the closer to the cell edge area }\end{array}$} \\
\hline UE speed $(\mathrm{km})$ & \multicolumn{2}{|c|}{$3,5,10$ and 30} \\
\hline MeNB to PeNB distance & \multicolumn{2}{|c|}{ ISD $>100 \mathrm{~m}$} \\
\hline PeNB to PeNB distance & \multicolumn{2}{|c|}{ ISD $>50 \mathrm{~m}$} \\
\hline Macro Cell Radius & \multicolumn{2}{|c|}{$500 \mathrm{~m}$} \\
\hline RSRP sample & \multicolumn{2}{|c|}{ Every $40 \mathrm{~ms}$} \\
\hline TTT & \multicolumn{2}{|c|}{$160 \mathrm{~ms}$} \\
\hline A3 offset & \multicolumn{2}{|c|}{$3 \mathrm{~dB}$} \\
\hline CoMP threshold & \multicolumn{2}{|c|}{$6 \mathrm{~dB}$} \\
\hline Handover preparation time & \multicolumn{2}{|c|}{$50 \mathrm{~ms}$} \\
\hline MTS & \multicolumn{2}{|c|}{$\leq 1$ second } \\
\hline
\end{tabular}

In order to be able to analyze the potential of the EHoLM handover procedure over conventional handover procedure, we have simulated both the EHoLM and the conventional handover process as mentioned in the previous section. We considered 
different simulation scenarios with varying numbers of PeNBs and users. The initial simulation assumptions are shown in Table 11 . We run 30 simulations for each of the scenarios and the simulation results are presented by considering a margin of error for $95 \%$ confidence interval. Some of the collected simulation results are presented below.

Figure 44 shows a comparison between the conventional and EHoLM with respect to the frequency of handover as a function of the number of UEs. In this case, we considered one macro cell with 24 pico cells as shown in Figure 43(a) and a different set of UEs (25, 50, 100 and 200). The speed of the UEs is considered $3 \mathrm{~km} / \mathrm{h}$ and the UEs move at random directions over the coverage area. The simulation time for all the four sets of UEs is the same. In 44(a), both the conventional and EHoLM handover procedure use the same carrier frequency of $2000 \mathrm{MHz}$ for macro and pico eNBs as suggested in [9]. In 44(b), we use the same carrier frequency $2000 \mathrm{MHz}$ for macro and pico eNBs in the conventional approach, but carrier frequencies of 2000 and $3500 \mathrm{MHz}$ for EHoLM. In 44(c), both conventional and EHoLM use different carrier frequency $2000 \mathrm{MHz}$ and $3500 \mathrm{MHz}$ for macro and pico eNBs respectively as suggested by 3GPP in [9]. All the three cases show that EHoLM reduces the number of handovers significantly. 


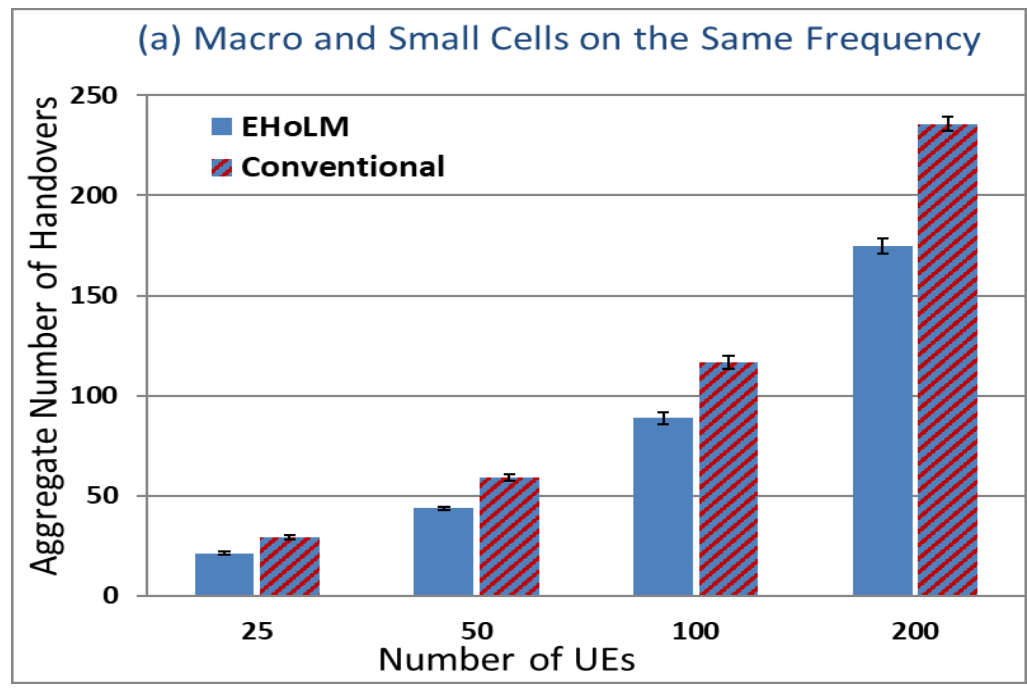

(b) Macro and Small Cells on Same Frequency in Conventional Approach but Different Frequency in Proposed Approach
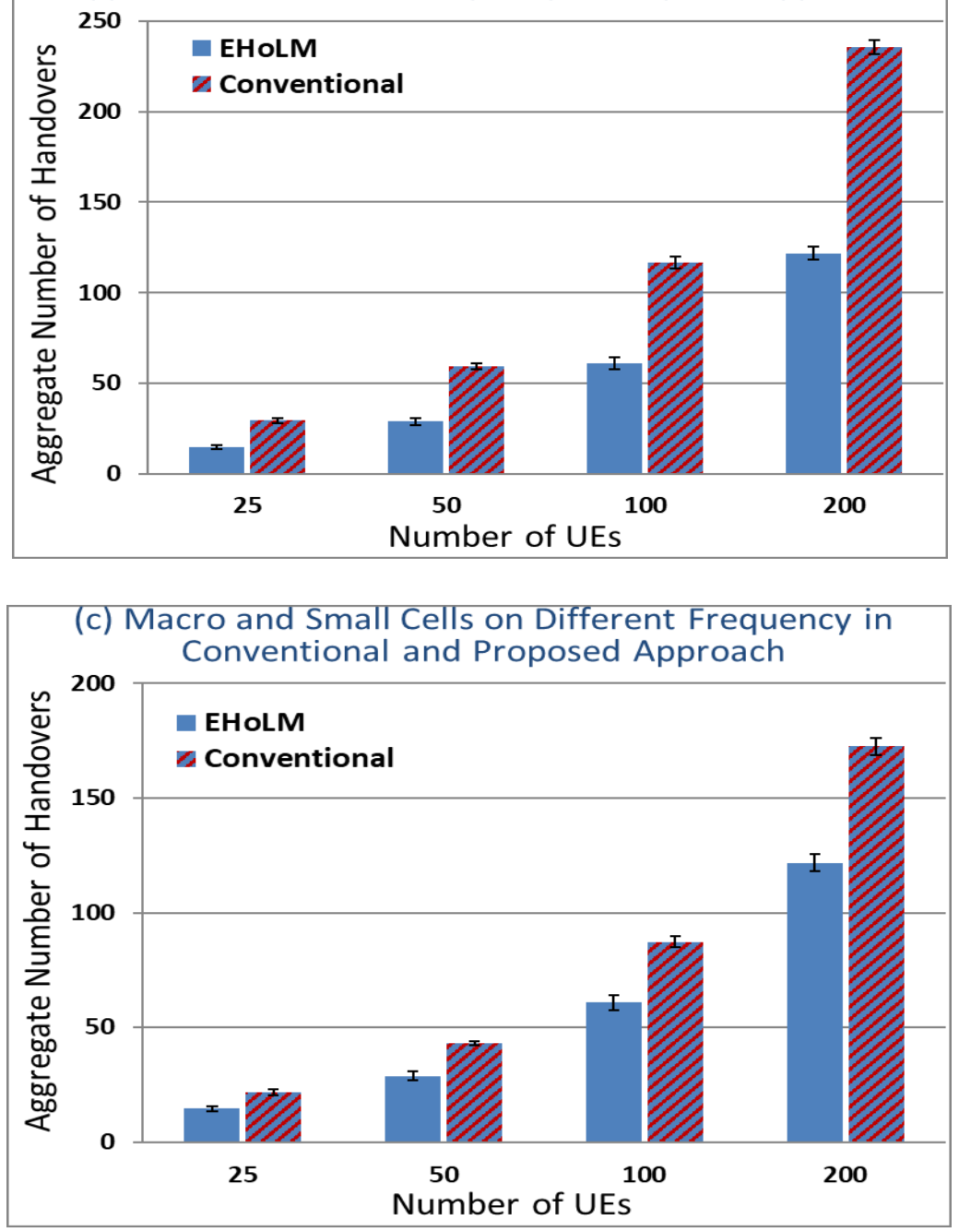

Figure 44: Number of handovers with respect to the number of UEs 
Figure 45 shows a comparison between the conventional and EHoLM with respect to the number of handovers as a function of the UE speed. The simulation scenario uses 19 macro cells, 72 Pico cells as shown in Figure 43(c) and 200 UEs. The speed of the UEs is considered $3,5,10,20$ and $30 \mathrm{~km} / \mathrm{h}$. The UEs move in random directions over the simulation area from their current position to their destination. In 45(a), both the conventional and EHoLM handover procedures use the same carrier frequency of 2000 $\mathrm{MHz}$ for macro and pico eNBs as suggested by 3GPP for HetNet scenario 1 [9]. In 45(b), both the conventional approach and EHoLM use different carrier frequencies of 2000 and $3500 \mathrm{MHz}$ for macro and pico eNBs respectively as suggested by 3GPP for HetNet scenario 2 [9]. In 45(c), we use the same carrier frequency of $2000 \mathrm{MHz}$ for macro and pico eNBs in the conventional approach but carrier frequencies of $2000 \mathrm{MHz}$ and $3500 \mathrm{MHz}$ in EHoLM. All the three cases with different UE speed show the EHoLM handover procedure reduces the number of handovers significantly. 


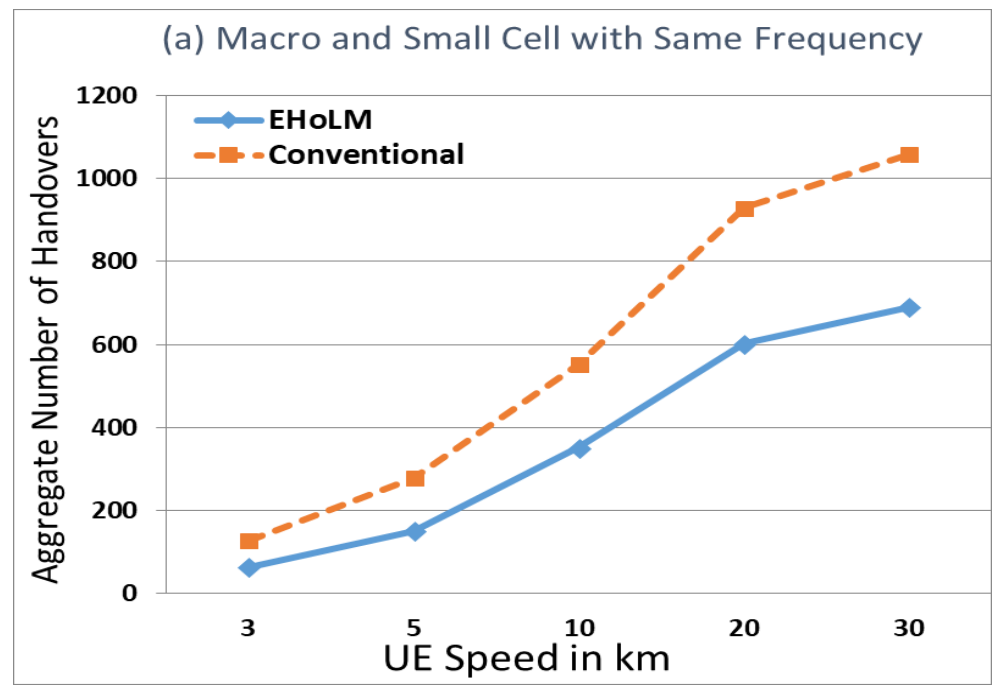

(b) Macro and Small Cells With Different Frequency in

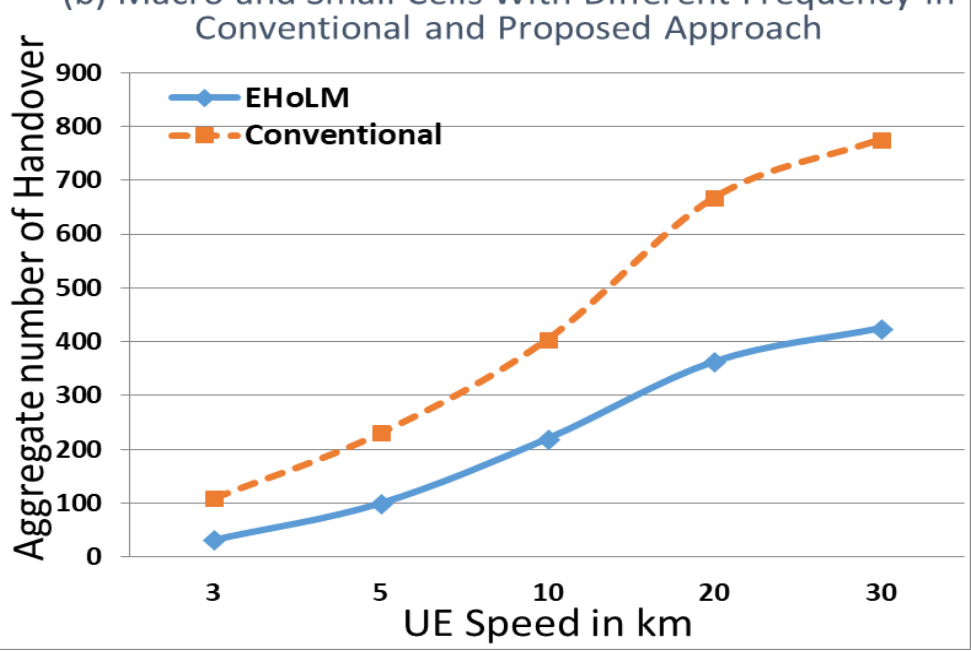

(c) Macro and Small Cells on Same Frequency in Conventional Approach but Different Frequency in Proposed Approach

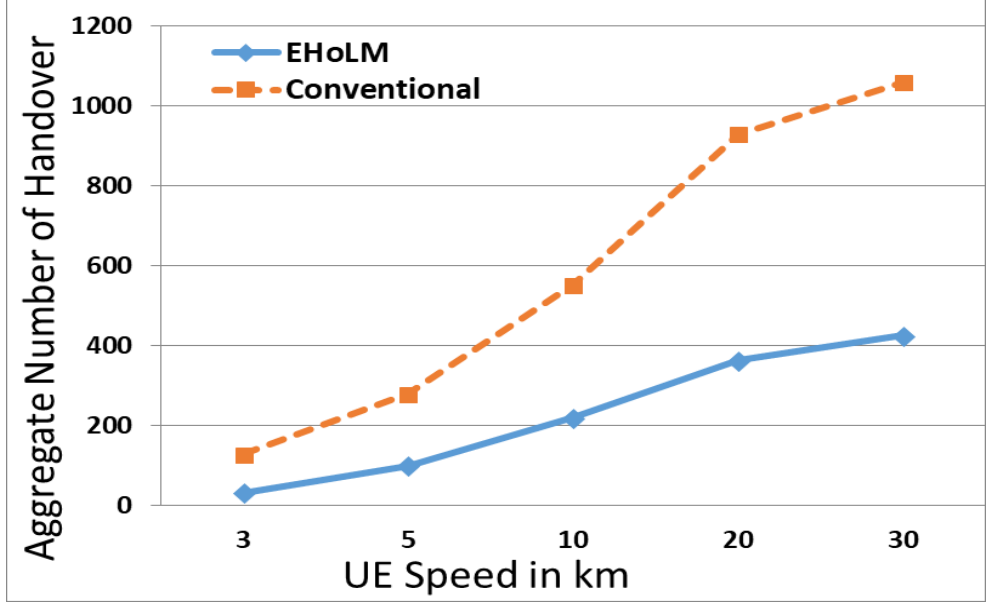

Figure 45: Number of handovers with respect to UE speed 
Figure 46 shows the number of handovers required for each of the UE in EHoLM and the conventional handover approach. In this case, we also used 19 macro cells, 72 Pico cells and 200 UEs. The blue triangles and the orange circles represent the same UEs in conventional and EHoML approaches respectively. The same UE shifted its position in the graph based on the number of handovers in two different approaches. If we look at the trend lines, it shows that EHoLM reduces the number of handovers about $50 \%$ than the conventional approach.

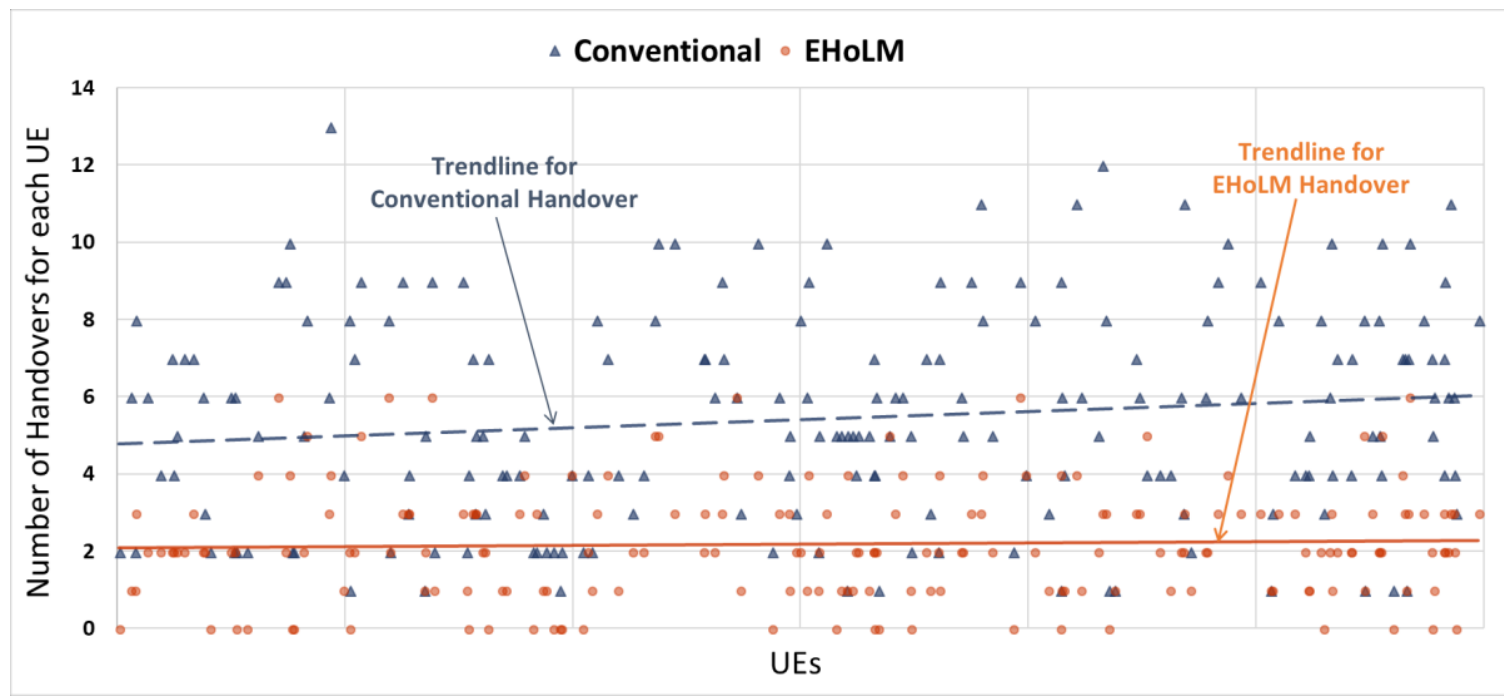

Figure 46: Number of handovers with respect to each of the UEs

For farther evaluation, we increase the network density as densification of the network is considered one of the key enablers to achieve the goal of the next generation wireless networks. Here we considered a network of $7 \mathrm{MeNB}$ and $16 \mathrm{PeNB}$ in each of the macro cells as shown in Figure 43(b). The number of UEs is 200 and distributed randomly all over the network area. The UEs move in random direction within the network. We considered same carrier frequency (SCF) of $2000 \mathrm{MHz}$ for both macro eNBs and pico eNBs, and different carrier frequencies (DCF) of $2000 \mathrm{MHz}$ for the macro eNBs and $3500 \mathrm{MHz}$ for the pico eNBs. Figure 47 shows that in all of the cases EHoLM scheme 
reduces the number of handovers significantly in the dense heterogeneous networks as well.

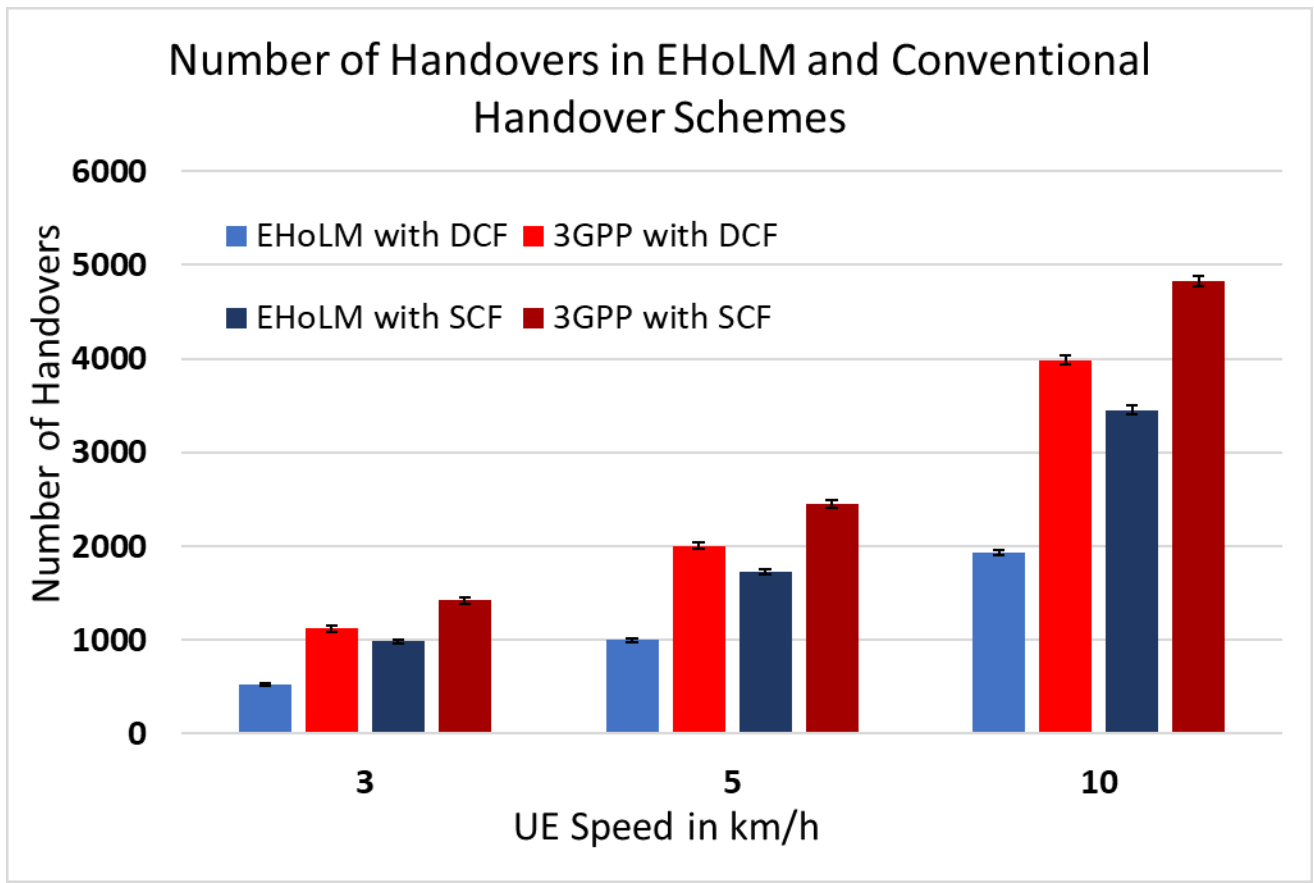

Figure 47: Number of handovers in EHoLM and Conventional handover schemes with respect to the UE speed and carrier frequencies

According to the simulation results, shown in Figures 44 to Figure 47 we can see that EHoLM has the potential to reduce the number of handovers, which is one of the main performance metrics for evaluating the handover process in heterogeneous cellular networks.

Figure 48 shows a comparison between the conventional and EHoLM scheme with respect to the number of handover oscillations as a function of UE speed. The simulation scenario uses 1 macro cell, 24 Pico cells as shown in Figure 43(a). In this case we considered 100 UEs. The UEs are randomly distributed closer to the cell edge area of macro eNBs and pico eNBs. The speed of the UEs is considered 3, 5, 10 and $30 \mathrm{~km} / \mathrm{h}$. In $48(\mathrm{a})$, both the 
conventional and EHoLM handover procedures use the same carrier frequency of 2000 $\mathrm{MHz}$ for both macro and pico eNBs. In 48(b), both the conventional approach and the EHoLM scheme use different carrier frequencies of 2000 and $3500 \mathrm{MHz}$ for macro and pico eNBs respectively. According to the figure, in this case of different carrier frequencies for MeNBs and PeNBs, EHoLM reduces the number of handover oscillations more than same carrier frequencies for MeNBs and PeNBs compared to conventional handover process. However, in both of the cases with different UE speed, the EHoLM handover procedure reduces the number of handover oscillations significantly.

\section{Number of handover oscillations}

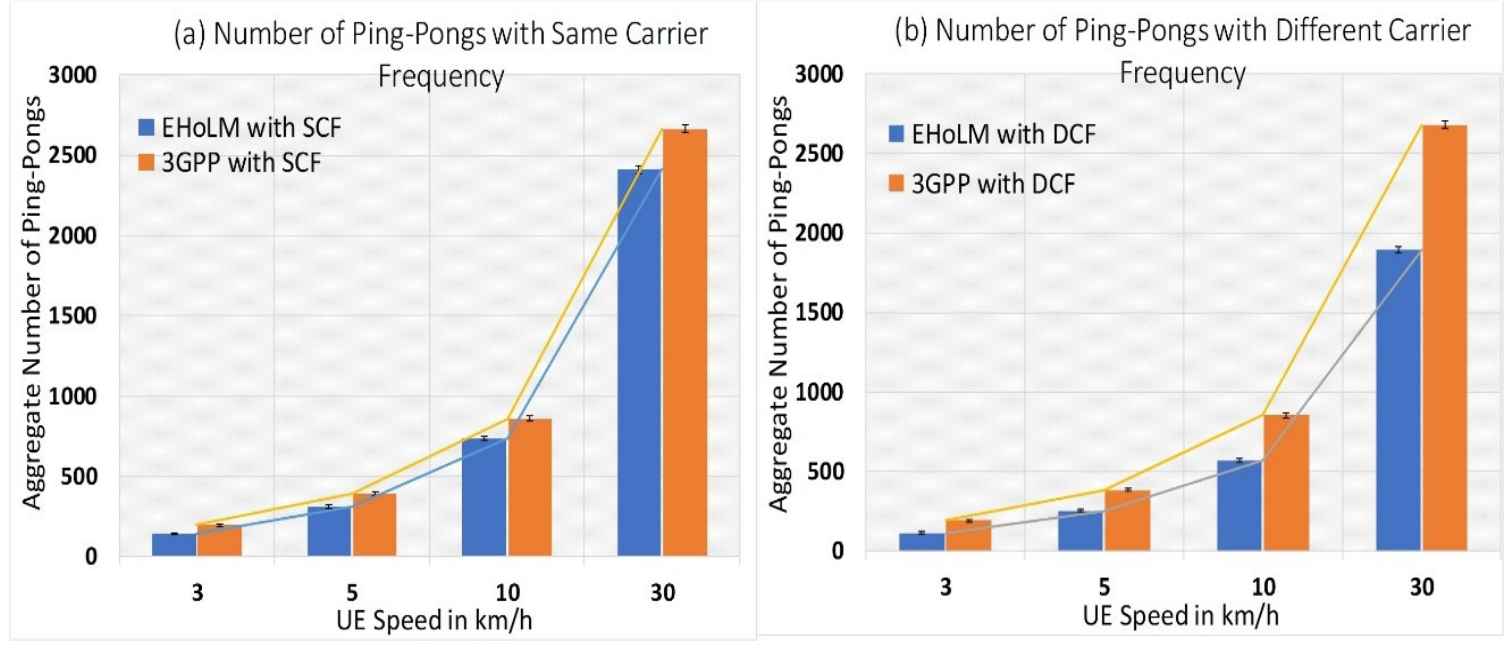

Figure 48: Number of handover oscillations with respect to the UE speed

Figure 49 shows the percentage of UEs participate in the handover or handover oscillation in the conventional and EHoLM scheme. In this case, we use the same network and the same set of UEs for both conventional and EHoLM scheme. According to the figure, $12 \%$ 
of the UEs do not require to participate in the handover oscillation in EHoLM scheme. This results again prove the improvement of EHoLM over the conventional approach.

Percentage of the UEs do not participate in the oscillation

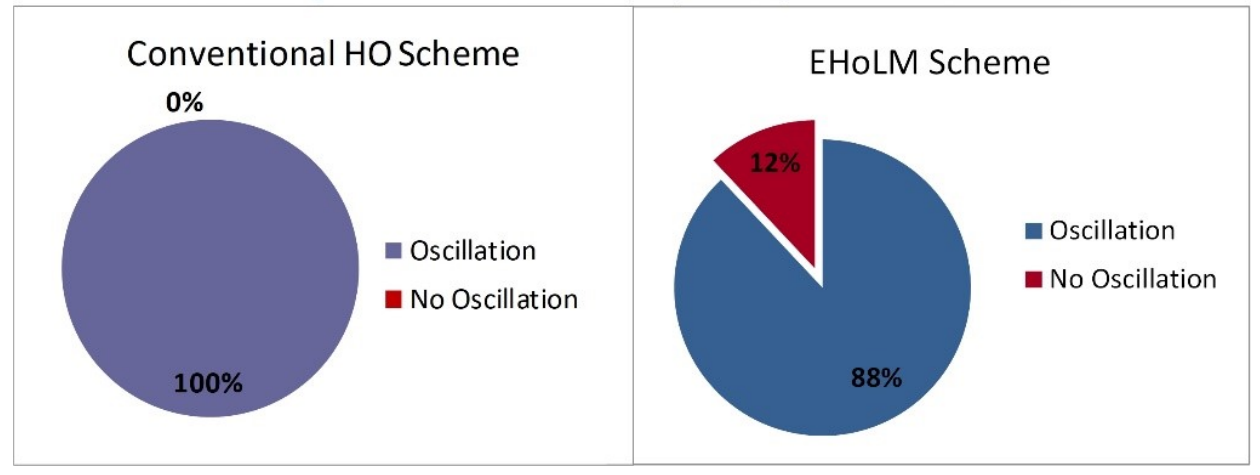

Figure 49: Percentage of UEs do not participate in the handover process

According to the simulation results, shown in Figures 44 to Figure 49 we can see that EHoLM has the potential to reduce the number of handovers and handover oscillations. Number of handovers and handover oscillations are the two key performance metrics for evaluating the handover process in cellular networks. The reduction of handovers and handover oscillations will reduce the signaling overhead and switching load within the cellular network. These overheads are directly impacts to the system performance. Therefore, EHoLM could improve the performance of cellular networks and user experience.

\subsection{Summary}

The main goal of this research is to improve the UE mobility so that network performance could be improved and users could get a better experience. EHoLM is tested in different heterogeneous scenarios as mentioned in the previous sections of the thesis. We have 
shown that this approach reduces the number of handovers compared to the conventional approach. The simulation results also showed that this approach reduces the number of handover oscillations compared to the conventional handover approach. The reduction of the number of handovers and oscillations reduce the control overhead within the network. Therefore, EHoLM has the potential to improve the overall performance of wireless cellular networks. In addition, the reduction of the handover failure improves the users' experience. The potential future improvement of EHoLM approach has been discussed in Chapter 6. 


\section{Chapter: Verification and Validation of Simulation Models}

The primary process for ensuring the credibility and accuracy of the computational results of a simulation model is known as verification and validation $(\mathrm{V} \& \mathrm{~V})$. The purpose of this chapter is to provide a general understanding of how a simulation model can be verified and validated. We propose a common framework that accommodates both the conceptual and formal approaches of the $\mathrm{V} \& \mathrm{~V}$ process. The study also aims to present where conceptual and formal V\&V process fit into the overall modeling and simulation (M\&S) lifecycle. Moreover, we also present how we validate the simulation models we developed and used in our research in the earlier two chapters.

\subsection{Verification and Validation Framework}

Before discussing the proposed V\&V framework, we present briefly some difficulties of the verification and validation process of a simulation model. First, a model is only validated with respect to its objective or within an experimental frame (EF). A model valid for one EF cannot be assumed that it is valid for another EF even though the system of interest is same. We will discuss more details about EFs later in this section. Secondly, validation often involves a comparison with the historical data of a real system that a model runs under the same conditions of the real system. This real system data is actually a sample data, which in itself creates some level of inaccuracy. Moreover, the performance of a simulator often correlated with the complexity (scope, number of states per component etc.) of a model. We might need to simplify a model to execute on a resource-limited simulation environment that reduce the validity of the model [135]. Therefore, there is always a trade-off between performance and V\&V is needed. Furthermore, a model could 
be developed on a proposed system. In that case, there is no real-world data to validate the model and we should depend on the expected outcome. Finally, there might not enough time to validate and verify every aspect of a model and its simulator [136]. As a result, it is not possible to prove that a model is absolutely correct, but we should consider a certain level of accuracy within the experimental frame or objective.

Moreover, the techniques of $\mathrm{V} \& \mathrm{~V}$ could be formal (quantitative, mathematical proofs) or conceptual/informal (subjective, qualitative, human reasoning), and each of the approach has its own advantages and difficulties $[137,138]$. It is difficult to apply all of the V\&V techniques to every model and corresponding simulator. For example, in case of large highly complex system, formal verification is difficult and extensive testing based on different test cases is suggested [29]. Furthermore, in [137], the author mentioned that model validation cannot be absolute and entirely formal.

Keeping in mind all of the above issues, in this section we presented a common framework for the $\mathrm{V} \& \mathrm{~V}$ process of a simulation model as shown in Figure 50. In this framework, we tried to accommodate both informal and formal techniques of the $V \& V$ process as the ultimate objective is to improve the accuracy and gain the users' confidence. 


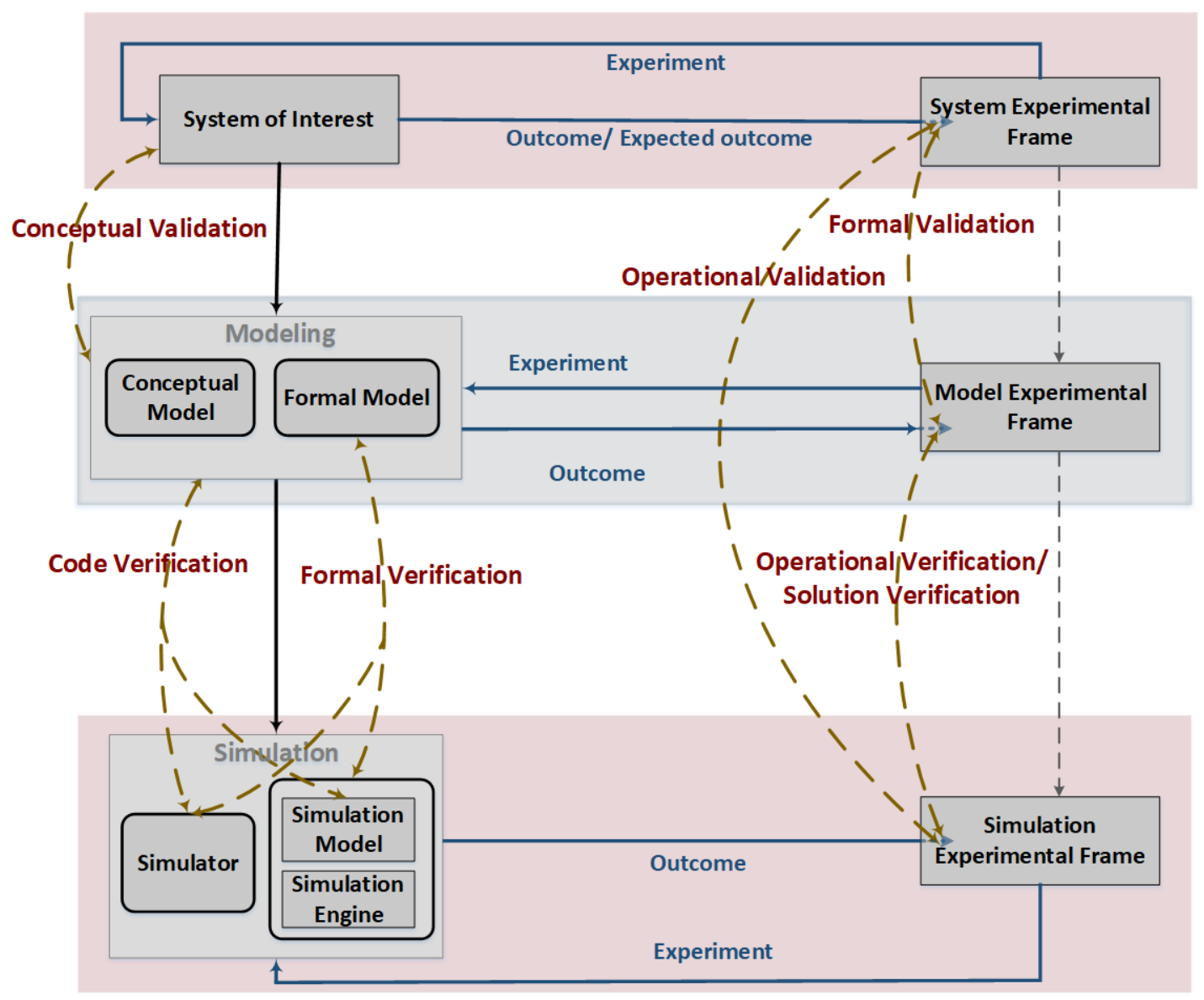

Figure 50: A common framework for verification and validation process

In the proposed framework, system of interest is a real system or a virtual system. The system is observed or experimented based on the system experimental frame (SyEF). The system experimental frame (SyEF) is a specification of the conditions according to the objective under which a system will be observed or experimented with. Oren and Ziegler in [139] defined five types of information to be contained in an experimental frame. The information that should have into the $\mathrm{EF}$ are: observational variables, input schedule, initialization settings, termination condition and collection of simulation data and display. Later, Daum and Sargent in [140] also discussed EF and suggest adding one more 
component such as system structure. In this thesis, wireless cellular network is our system of interest. For example, following are some of the information of the SyEF we defined for our experiment to analyze the handover process in wireless cellular networks:

Observational variables: number of handovers, number of ping-pongs and handover failure rate.

Input schedule: measurement report (MR) triggering rate, TTT rate and UE speed.

Initialization settings: MeNB transmit power $43 \mathrm{~dB}$, PeNB transmit power $30 \mathrm{~dB}$, etc.

Termination condition: predefined runtime.

Collection of simulation data and display: 95\% confidence interval, histogram, and line chart etc.

A model of a real system is designed to conduct experiments for better understanding the behavior of the system. We can design a conceptual model or a formal model or both. A conceptual model is an abstract representation of the system of interest. A conceptual model is an informal description of the system to communicate with the diverse groups, participating in the $M \& S$ process. It designs to provide the inside of a system and how it works in general. A conceptual model may be written using natural language. However, a conceptual model is extremely useful to describe a technically advanced system to someone who is not familiar with the system details. On the other hand, a formal model is a representation of the system dynamics using a formal method such as automata theory, DEVS formalism etc. It is an unambiguous description of model structure. A formal model provides better services for model testing, modification and reuse. A formal approach can reduce the ambiguity and enhance teamwork, which ultimately reduces the development 
time and cost. More importantly, a formal method also provides mechanisms to formally validate and verified a model. In this thesis we used DEVS formalism to model wireless cellular networks to analyze our proposed algorithms discussed in the earlier chapters. Figure 41 shows the DEVS model for handover process in heterogeneous wireless cellular networks.

The content of the experimental frame could be different in the modeling domain as we show in Figure 50. In this framework, the experimental frame for a model is called as model experimental frame (MoEF). The MoEF tries to mimic the experiments defined in the SyEF on the original entity to obtain the desired results [33].

A simulator is a set of instructions that capable of executing a model to generate its expected behavior or produce the results. A simulator could be one entity integrating simulation model and simulation engine or two separate entities, simulation engine and simulation model as shown in Figure 50. The second option could provide more flexibility and robustness in case of model implementation as it provides complete separation of simulation engine and simulation model. In our research, we used the separation of simulation model and simulation engine. Figure 51 and 52 show the code snippet of the simulation model transformed from the DEVS model as shown in figure 41 for handover process in heterogeneous wireless cellular networks. In Figure 51, MeNBProc, PeNBProc and UEProc are the DEVS atomic models as describes in Section 4.4. Figure 52 shows the MeNB, PeNB and UE coupled models as shown in Figure 41. The simulation engine we used is the CD++ toolkit, implemented based on DEVS theory. 
The experimental frame for simulation is called as simulation experimental frame ( $\mathrm{SiEF})$.

The SiEF is derived from the MoEF so that the simulation model should be able to answer the same queries defined in the MoEF [33]. The separation of the three different experimental frames (SyEF, MoEF and SiEF), and formalize the model experimental frame (MoEF) and the simulation experimental frame (SiEF) could be a possible extension of this work.

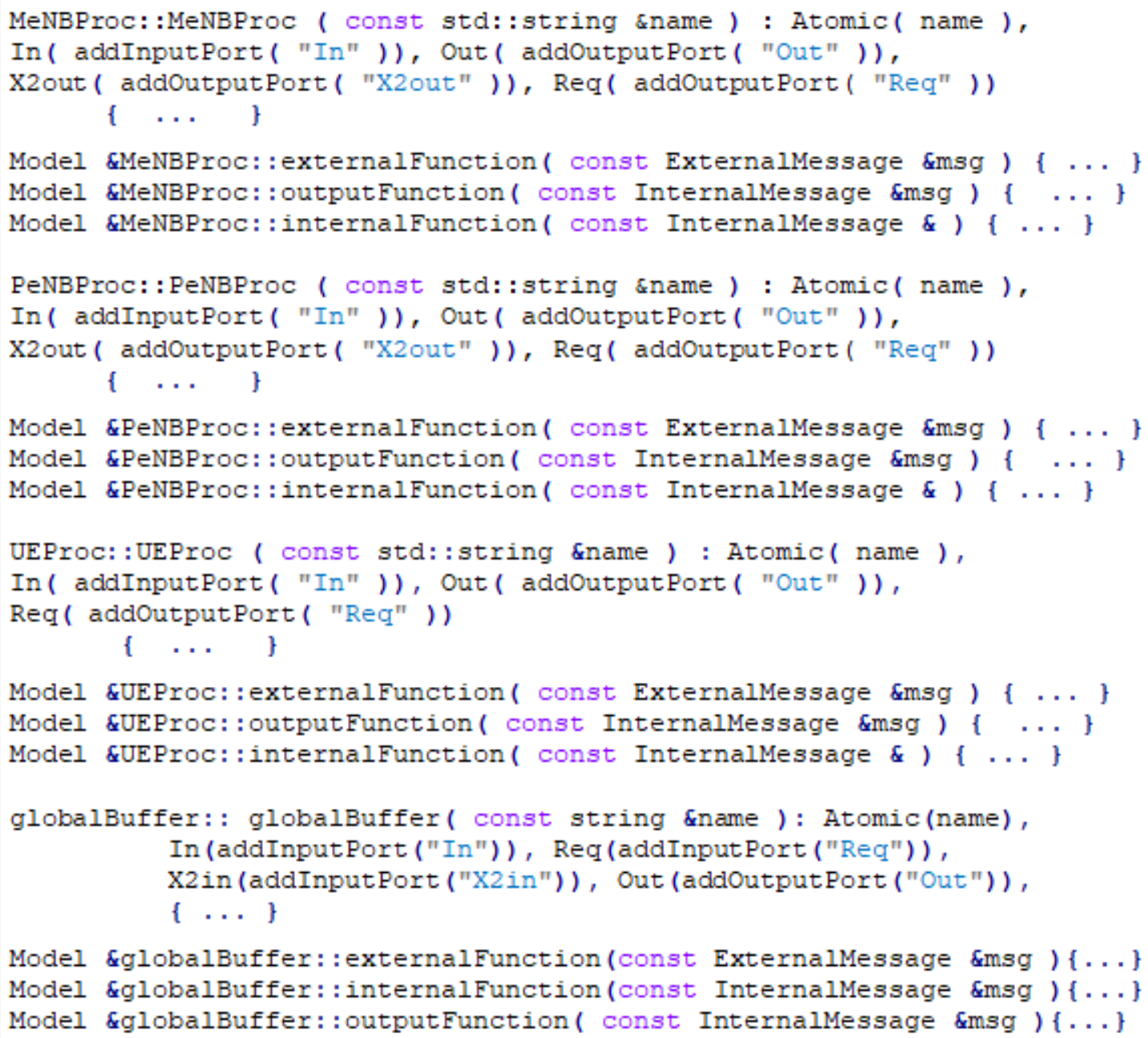

Figure 51: Sample code snippet of the simulation model (atomic models) developed from the DEVS model for handover process in heterogeneous cellular networks 


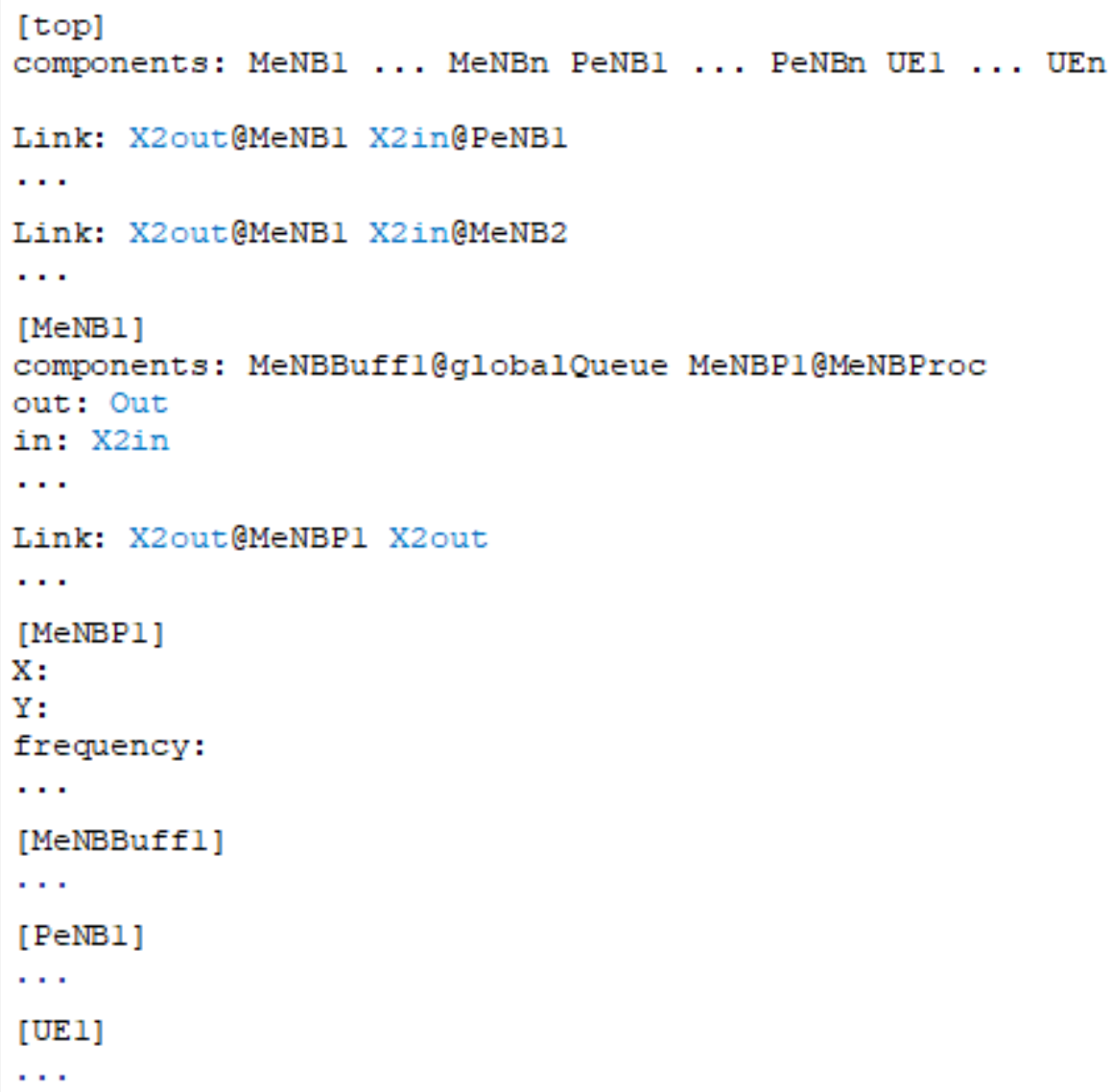

Figure 52: Sample code snippet of the simulation model (coupled models) developed from the DEVS model for handover process in heterogeneous cellular networks Verification process ensures that the simulator or the simulation model carries out the model instructions correctly. The verification process focuses on the identification and removal of errors in the implementation (simulation model) of the system's model. On the other hand, validation is the process to establish the validity of the structure of a model and the accuracy of the behavior of a model to reproduce the behavior of the system of interest according to the objective of the study. V\&V process could be conceptual/informal and formal as we stated before. Conceptual validation determines that the model holds the necessary details (system structure, input-output data etc.) representing the system of 
interest to meet the objective of the study $[136,105]$. It placed between the system of interest and the model as shown in Figure 50. Formal validation, on the other hand checks the validity of a formal model according to the experimental frame defined from the objective of the study. In the formal validation approach, three steps are replicative, predictive and structural. Replicative validation is the basic level of validation process compares the input/output behavior of the model and the system. Predictive validity requires model and system agrees the input/output functions. Finally, structural validation compares the state transition and coupled components of the model and the system [29, 135]. Accordingly, validation could be conceptual/informal or formal or complementary as the ultimate objective is to establish the validity of the structure and the behavior of a model. The V\&V of a simulation model is a process of confidence building, improving the accuracy. Therefore, in the proposed framework, we accommodated both conceptual validation and formal validation approaches and present where they are suitable in the $\mathrm{M} \& \mathrm{~S}$ lifecycle as both of the approaches can be used complementary manner if required.

In the next section we discussed the verification and validation process considering the models we developed in our previous chapters to study the proposed algorithms in wireless cellular networks.

\subsection{Verification and Validation of Simulation Models}

In this section we discussed the V\&V techniques we used to verify and validate the simulation models we developed and used in our research. 


\subsubsection{Verification}

Verification is the process of confirming that a model has been transformed into a simulation model or simulator with acceptable accuracy according to the model's specification. According to the literature code verification and solution verification are the two key categories of verification process [141, 107, 29]:

Code verification ensures that the numerical algorithms or mathematical models are correctly implemented in the simulator and identify the errors into the code. The goal of code verification is to ensure that the code is a correct representation of the underlying model. The code verification is between the model and the simulator or the simulation model as defined in the framework and shown in Figure 50. There are a number of techniques are used for code verification, following are the two widely accepted activities that we used in our simulation model [107, 142]:

\section{(1) Trend test \\ (2) Code-to-code comparisons}

Trend test: In this method a set of calculation is performed by changing the input parameters to test whether the code can generate expected outcomes. The input and output can also be preset to the domain experts to get a judgment. In our work, we did trend test for all algorithms and mathematical models we used in our networks. For example, in the DCEC algorithm we randomly changed the eNB throughput to examine how the coordination station (CS) changes occurred. For propagation model we changed the transmit power of different types of eNBs and checked the signal strength in the different region of the network. We also changed the position of UEs in the network and observed 
that the UEs can be connected to the right eNB based on the received signal strength. The test outcomes are examined by the Ericsson experts lead by Gary Boudreau and Ronald Casselman.

Code-to-code comparison test: another widely use approach is code to code comparison, in which the output of one code is compared to the output of another code. In this test we checked the output of our propagation model to the output of the propagation model of previously completed research work in the area of wireless networks in our lab and the other research works in this area. The average number of eNBs in a CoMP cooperation set is also compared and matches to the other related research works.

Solution verification starts after the code verification of a simulator. It checks the accuracy of the simulations outcome compared to the model outcome. Solution verification mainly focuses on the verification of the numerical accuracy of the solution obtained [107]. To verify the numerical accuracy, we considered round-off error and iterative convergence error. To reduce the round-off error and iterative convergence error we specify the precision depending on the programming language we used $(\mathrm{C}++)$, as suggested in [143]. We use rounding very carefully such as we did rounding for calculating the position (coordinates) of eNBs and UEs that does not affect our concern output of the simulation.

Moreover, we used the $\mathrm{CD}++$ simulation engine that implements DEVS formal specification. The $\mathrm{CD}++$ toolkit is a formally verified simulation engine. Therefore, we are more concern about the code verification and the solution verification of simulation model rather than formal verification in this case. We also use DEVS formal specification for the simulation models we developed to analyze the cellular networks employing our proposed 
algorithms as we said before. The use of formal model and formally verified simulation engine enables automated simulation model verification [144].

\subsubsection{Validation}

Validation is the process of determining the degree to which a model is an accurate representation of the real system from the perspective of the intended uses of the model as we stated before. Naylor and Finger formulated a three steps validation process that has been widely followed [100]. Moreover, Sargent in [105, 145] and Robinson in [136] also discussed validation techniques those are generally used. In this thesis we considered following validation techniques suggested in the above-mentioned articles.

Face validation: The first step of a model validation is that the model should appear reasonable to potential model users and domain experts. We presented our models and overall input-output of the model to the Ericsson experts to get their feedback. We also presented any improvement of our model often to the other researchers in our lab.

Validation of model assumptions: Related to the collection of reliable assumptions and statistical analysis. This is also known as data validation. All of our simulation assumptions in both the DCEC CoMP coordination architecture and the EHoLM handover approach are based on the 3GPP specifications and other state-of-the-art related works, and we cited that in the thesis. For example, Table 12 shows the simulation assumptions and corresponding references for each of the parameters we assumed in EHoLM approach. Moreover, in case of user arrival and user distribution in the networks we considered probability distributions suggested in the 3GPP specifications and verified with the Ericsson research team as we mentioned before.. 
Table 12: Simulation assumption and corresponding references

\begin{tabular}{|c|c|c|}
\hline Parameters & Value & References \\
\hline Number of MeNBs & $3,7,19$ & {$[32,9,68]$} \\
\hline Number of PeNBs & $\begin{array}{l}6 \text { per macro cells (Hexagonal border of } \\
\text { each macro cells) and } 10 \text { per macro cells }\end{array}$ & {$[32,9]$} \\
\hline MeNB Transmit power & $43 \mathrm{dBm}$ & {$[32,9]$} \\
\hline PeNB Transmit power & $30 \mathrm{dBm}$ & {$[32,146,9]$} \\
\hline Carrier Frequency & $2000 \mathrm{MHz}$ & {$[9,146]$} \\
\hline & $3500 \mathrm{MHz}$ & [9] \\
\hline Path loss model & $128.1+37.6 \log _{10}(d)$ & {$[32,147,9]$} \\
\hline Path loss model & $147+36.7 \log _{10}(\mathrm{~d})$ & {$[32,147,9]$} \\
\hline UE speed $(\mathrm{km})$ & $3,5,10$ and 30 & {$[32,9,148]$} \\
\hline MeNB to PeNB distance & ISD $>100 \mathrm{~m}$ & {$[9,27]$} \\
\hline PeNB to PeNB distance & ISD $>50 \mathrm{~m}$ & {$[9,27]$} \\
\hline Macro Cell Radius & $500 \mathrm{~m}$ & {$[35,9]$} \\
\hline RSRP sample & Every $40 \mathrm{~ms}$ & {$[32,9,149]$} \\
\hline TTT & $160 \mathrm{~ms}$ & $\begin{array}{c}{[32,9,149,129,} \\
148,128]\end{array}$ \\
\hline A3 offset & $3 \mathrm{~dB}$ & {$[32,149,129]$} \\
\hline CoMP threshold & $6 \mathrm{~dB}$ & {$[111,68,112]$} \\
\hline Handover prep. time & $50 \mathrm{~ms}$ & {$[32,9,129]$} \\
\hline MTS & $\leq 1$ second & {$[32,9,129]$} \\
\hline
\end{tabular}


Degenerate test: In this technique a model is tested by selecting the appropriate value of the input and internal parameters. In our models we checked how the eNB queue behaves with the increase or decrease of the CSI feedback periodicity or density of the UEs in the networks.

Extreme condition test: Checks the reasonability of the outputs for any extreme factors. In our simulation models we checked if a UE is in stationary, does it trigger or not the measurement report (MR) to the serving eNB.

Event validation: Determine the similarity of occurrence of events considered in the model to the real system. For example, in our simulation model we considered a UE calculates measurement report (MR) in every $40 \mathrm{~ms}$ and CSI feedback periodicity is $5 \mathrm{~ms}$ or $10 \mathrm{~ms}$ based on the industry specification.

As we stated before, in this thesis we presented a novel coordination architecture for multicell cooperative communication to improve the performance of wireless cellular networks by reducing signaling overhead and delay. Employing new architecture into the networks could consider a proposed system and we don't have real-world data to be validated. The situation like this, comparing the simulation against the mathematical model is suggested, as a mathematical model is able to predict the outcome of the simulation model [136]. Therefore, we developed simplified mathematical models to calculate the number of control messages related to CSI feedback transmit into the networks for all the three CoMP coordination architectures (Centralized, Distributed and DCEC). We compared the simulation outcome to the mathematical model outcome. This approach can be considered as operational validation or replicative validation which is the basic level of formal 
validation approach. Message transmission is considered because of its high importance in the communication networks. Following are the mathematical models we develop to calculate the number of CSI feedback messages for centralized, distributed and DCEC CoMP coordination architectures.

$$
\begin{aligned}
& N_{C S I-f b_{C e n}}=\sum_{\Delta t=1}^{T} \sum_{i=1}^{n}\left(\left(N_{f b_{U E_{i \Delta t}-e N B s_{i}}}+N_{f f_{e N B s_{i \Delta t}-C U}}\right)\right. \\
& \left.+\left(N_{\text {Sset }_{U E_{i}}} \cdot 2\right)\right) \\
& N_{C S I-f b_{D i s}}=\sum_{\Delta t=1}^{T} \sum_{i=1}^{n}\left(N_{f b_{U E_{i \Delta t}-e N B s_{i}}}\right. \\
& \left.+\left(N_{f f_{e N B s_{i \Delta t}-e N B c_{i}}}\right) \cdot\left(N_{C s e t_{U E_{i}}}-1\right)\right) \\
& N_{C S I-f b_{D C E C}}=\sum_{\Delta t=1}^{T} \sum_{i=1}^{n}\left(\left(N_{f b_{U E_{i}-e N B s_{i}}}+\left(N_{m s g-C S_{U E_{i}}}\right) \cdot N_{C s e t_{U E_{i}}}\right)\right. \\
& \left.+N_{f b_{U E_{i \Delta i}-C S_{U E_{i}}}}\right)
\end{aligned}
$$

Equation 21

Equation 22

In the above equations, $N_{C S I-f b_{C e n}}$ is the number of control messages related to CoMP cooperation in the centralized architecture, $N_{C S I-f b_{D i s}}$ in the distributed architecture and $N_{C S I-f b_{D C E C}}$ in the DCEC architecture. $\Delta t$ is the number of time steps, which is depends on the CSI feedback periodicity. The number of UEs in the CoMP operation is represented 
by $i . N_{f b_{U E_{i \Delta t}-e N B s_{i}}}$ is the number of feedback messages from $U E_{i}$ to its serving eNB at $\Delta t$. $N_{f f_{e N B s_{i \Delta t}}{ }^{-C U}}$ is the number of CSI feedback forward messages from serving eNB to CU in case of centralized architecture at time step $\Delta t . N_{C S e t_{U E_{i}}}$ is the number of eNBs in the CoMP cooperation set for $U E_{i} . N_{f f_{e N B s_{i \Delta t}-e N B c_{i}}}$ is the number of CSI feedback forward messages from serving eNB to cooperating eNBs of $U E_{i}$ in distributed architecture. $N_{m s g-C S_{U E_{i}}}$ is the number of messages to elect a coordination station (CS) for $U E_{i}$ in the

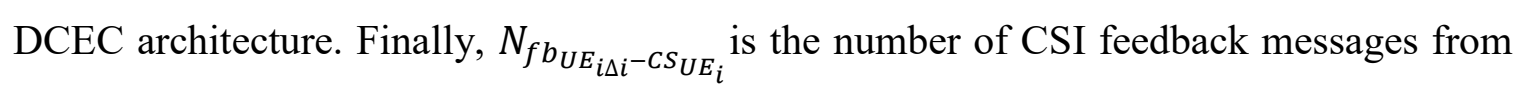
$U E_{i}$ to CS in each time step. 
(a) Comparison of Mathematical and Simulation Models

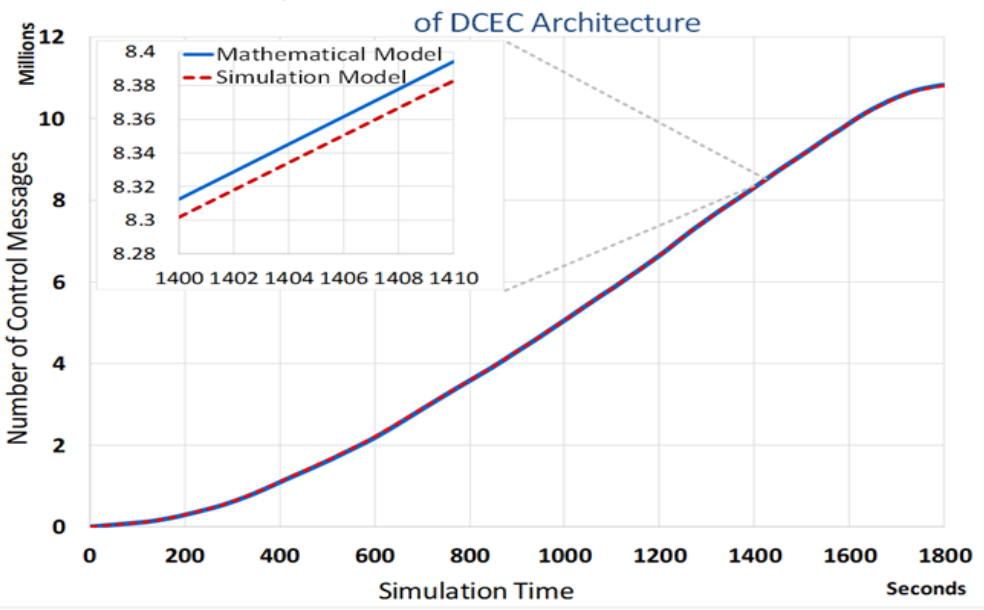

(b) Comparison of Mathematical and Simulation Models

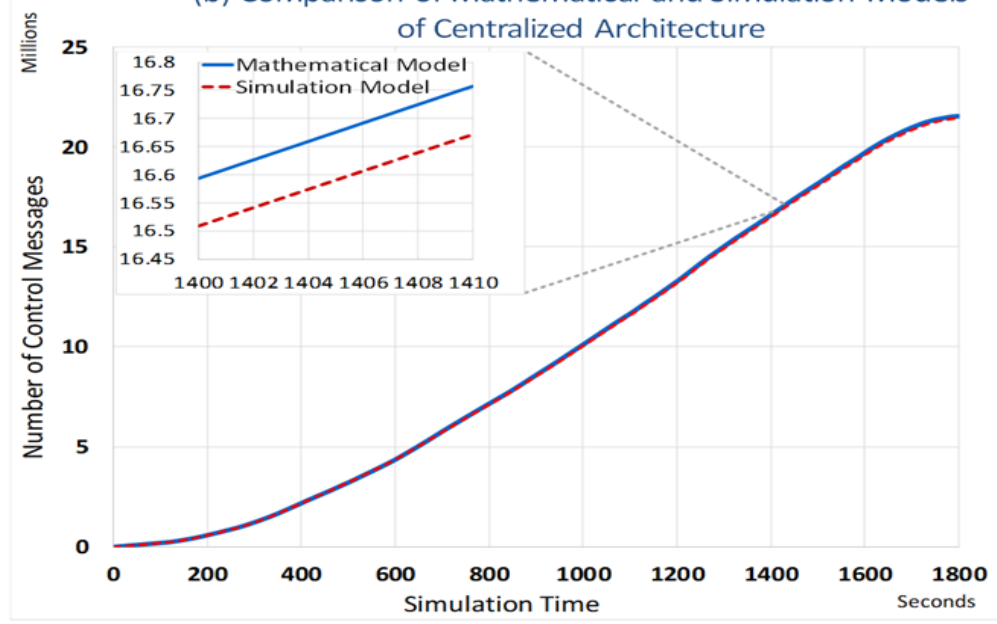

(c) Comparison of Mathematical and Simulation Models

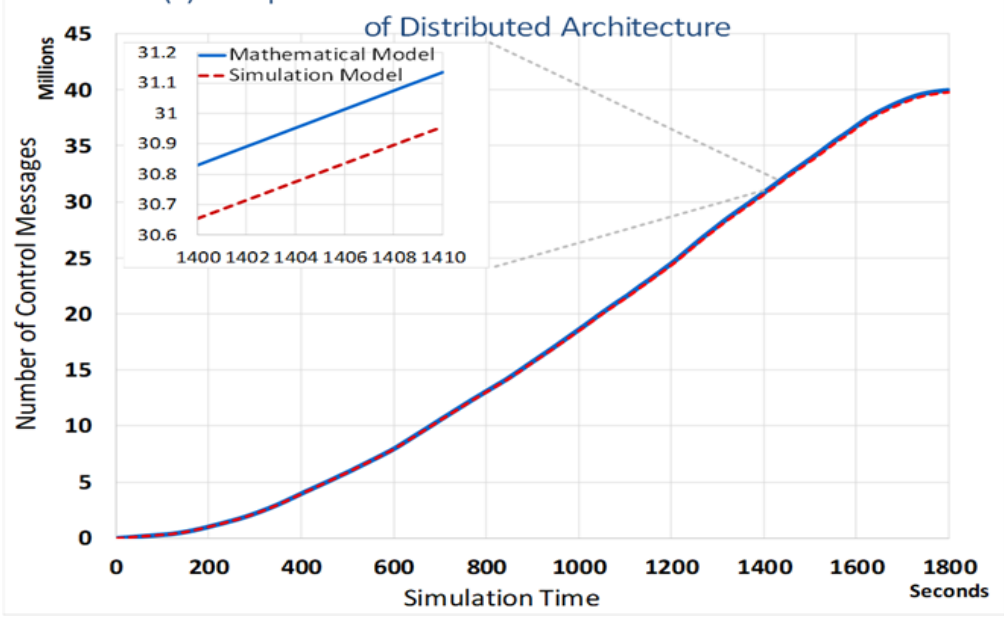

Figure 53: Comparison of mathematical model and simulation model for all of the three architectures 
In Figure 53 ( $a, b$ and c) blue lines represent the outcome of mathematical models and red dotted lines represent the outcome of the simulation models for DCEC, centralized and distributed CoMP coordination architectures respectively. We considered a network scenario with 7 macrocells and 10 picocells in each of the macrocells. In all the three cases we did message count every $500 \mathrm{~ms}$ for both mathematical and simulation models and plotted them. The horizontal axis shows the simulation time and the vertical axis shows the message count. To make it more clear, in the upper left corner of each of the graphs we magnify the area from simulation time $1400000 \mathrm{~ms}$ to $1410000 \mathrm{~ms}$. The difference between the outcome of the simulation model and the mathematical model in each of the architecture is about 0.34 to 0.45 percent, which is quite acceptable.

For farther validation of the handover model, we compare the simulation outcome (number of handovers) in homogeneous networks and heterogeneous networks, and confirmed that to the 3GPP specifications. The simulation scenario is considered 19 macrocells for homogeneous networks and 19 macrocells with 72 picocells for heterogeneous networks in the same coverage area. The pico eNBs are positioned at the border of macrocells as suggested by the 3GPP specifications to study HetNets. 


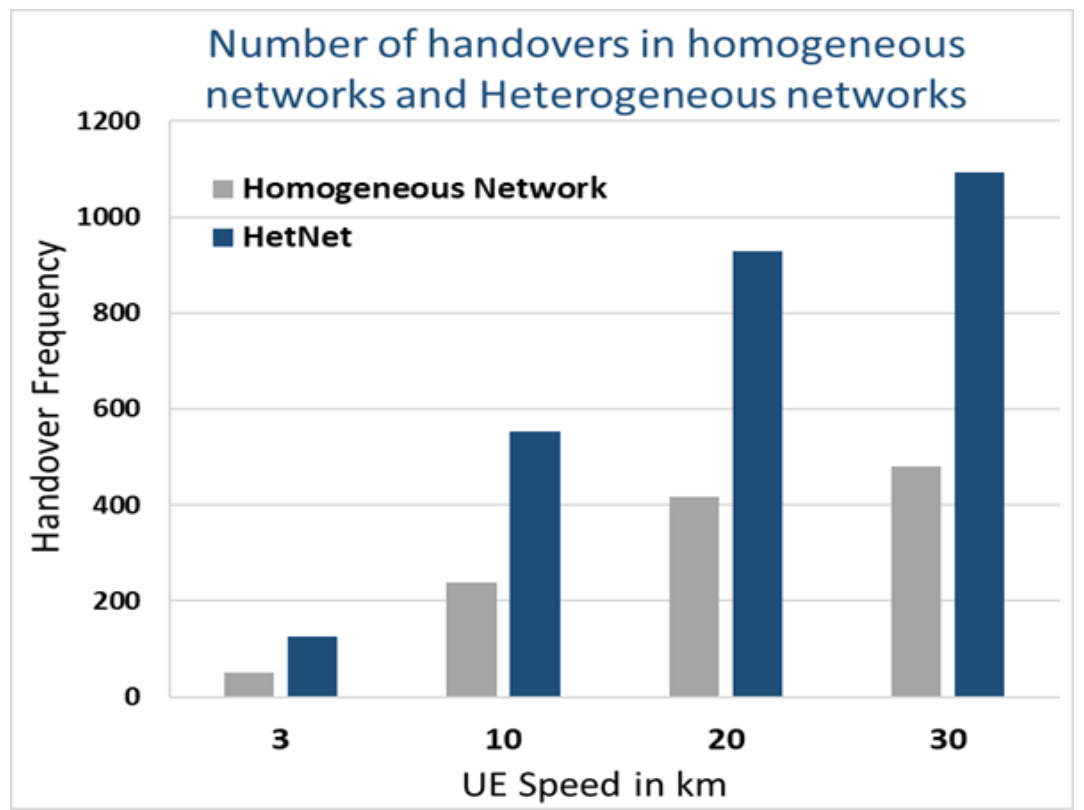

Figure 54: Number of handovers with respect to UE speed for homogeneous networks and heterogeneous networks

The simulation outcome is presented in Figure 54. In this figure, the gray bars represent the numbers of handovers in the homogeneous networks and the blue bars represent the number of handovers in the HetNets for different UE speeds. The simulation results show that the increase of handovers in HetNets compare to homogeneous networks is about $122 \%$ to $147 \%$ depending on the speed of UEs. The increase of the handovers in our simulation outcome agrees with the numbers $(120 \%-140 \%)$ mentioned in the $3 \mathrm{GPP}$ specification, telecommunication standardization body [9].

\subsection{Summary}

In this chapter we presented a common framework that accommodates both conceptual and formal approach of verification and validation process. The framework also shows where the conceptual and formal $\mathrm{V} \& \mathrm{~V}$ process fit into the modeling and simulation lifecycle. 
Moreover, we discussed several well-accepted V\&V techniques and presented how we verified and validate our simulation models based on these techniques. We want to extend the verification and validation process focusing on formal approach for the simulation models in the area of wireless networks. Moreover, we discussed the potential future works in the next chapter. 


\section{Chapter: Conclusion and Future Work}

This research investigates the issues of control plane signaling overhead in multicell cooperative wireless cellular communications. Several algorithms have been proposed to improve system performance reducing the signaling overhead. This chapter presents the conclusions of the research deducted from the results of the previous chapters and some prospective future research lines.

We addressed the signaling issues in multicell cooperative cellular networks, and presented novel control plane algorithms for multicell multitier cooperative cellular networks. We identified the open issues and challenges as presented in Chapter 2. Among these challenges, we focused on the CSI feedback overhead and latency, and on mobility management.

One of the contributions of this research work was to reduce the CSI feedback overhead (signaling overhead) and the latency for improving system performance. We presented two algorithms: a coordination architecture named DCEC (Direct CSI-feedback to Elected Coordination-station) for CoMP transmission, and an extended version for heterogeneous cellular networks named DCEC-HetNet. To study the CoMP operation employing the proposed coordination architectures, we built simulation models using the discrete event system specifications (DEVS) formalism and run simulations on various homogeneous and heterogeneous network scenarios. We also presented how DCEC and DCEC-HetNet reduce the CSI overhead and the feedback latency compared to other two CoMP coordination approaches. According to the simulation results, our new coordination architecture has the potential to improve the performance of the cellular networks reducing 
the signaling overhead and feedback latency. However, if the coordination station (CS) changes very rapidly, for example, every $100 \mathrm{~ms}$ or less, DCEC will be less efficient than the traditional approaches. Though, in practice, the CS change does not occur that frequently, since for CoMP deployment the speed of a UE suggested by the 3GPP release 11 and 14 is about $3 \mathrm{~km} / \mathrm{h}[12,27]$.

We also presented an extended handover procedure EHoLM (Enhanced Handover for Low and Moderate speed UEs) for dense heterogeneous cellular networks (Chapter 4). To study EHoLM we also built a simulation model using DEVS and run simulations considering several dense heterogeneous scenarios employing both EHoLM and a conventional handover procedure suggested in 3GPP specifications. The experimental results showed that the proposed handover approach has the potential to reduce the number of handovers and handover oscillations. This reduction improves system performance, as handover is one of the most resource-hungry procedures in cellular networks. The EHoLM handover procedure is not considered for high speed UEs.

A common framework for verification and validation process was presented in Chapter 5 . In this framework we accommodated both formal and conceptual approach of $\mathrm{V} \& \mathrm{~V}$ process. This framework also shows where different approaches of V\&V process fit in the modeling and simulation lifecycle. In this chapter, we also presented how we verified and validated the models we developed to study the algorithms we proposed. 
Following, we present some ideas that could be used as future extensions to our work.

The DCEC coordination architecture could be extended finding the optimal coordination station (CS) considering more parameters that better characterize the current status of an eNB in the context of ultra-dense heterogeneous networks (UDHetNets). This improved algorithm would provide us an optimal base station as well as might provide us enough information to mute base stations (eNBs). Muting an eNB will reduce the power consumption of the network as energy efficiency or green communication is one of the visions of information and communication technologies (ICT) for environmental sustainability of the future world.

Likewise, the EHoLM handover procedure needs deeper analysis on the handover failure rate. A further advancement of the EHoLM handover procedure could be to examine how it affects the power consumption of the user's equipment and the networks since the power consumption of a device also depends on the message transmission. As the EHoLM handover process reduces the control messages transmission within the networks, it might have the potential to improve energy efficiency (battery lifetime), which is one of the goals of the next generation wireless cellular networks.

The radio resource allocation in the control plane is gradually increasing in cellular networks that consume a significant amount of bandwidth. Radio frequency is the most scarce but precious resource in wireless cellular networks. Analyzing the wireless traffic dynamics employing data science algorithms for dynamic radio resource allocation could be another extension of our work. In this view, integrating data driven machine learning in the control plane to minimize the radio resource allocation in control plane could be a 
significant advancement in wireless networks.

Moreover, in the verification and validation framework we need to define and formalize the model experimental frame (MoEF) and the simulation experimental frame (SiEF) more clearly. A possible extension of this work is to define verification and validation test for the DEVS models in the area of wireless networks. Finally, the need for automated verification and validation tool is a big challenge in $M \& S$ lifecycle. Therefore, developing a formal validation and verification tool and incorporating that into the $\mathrm{CD}++$ toolkit could be a further advancement of this research. 


\section{Appendices}

\section{Appendix A Control Plane Protocol Overview}

The protocol architecture of the LTE and beyond cellular networks spans both user plane and control planes. The user plane is responsible for the transmission of actual data and voice. The control plane is responsible for a number of signaling functions to facilitate the user plane. The resources assigned for control plane in traditional wireless networks is about $5 \%$ of the total resources available in the system [150]. However, in new generation cellular networks need new approaches to improve the performance of the cellular networks, which requires exchanging more control information within the networks. Therefore, the requirement of resources for control signals also increasing and need to be managed very efficiently.

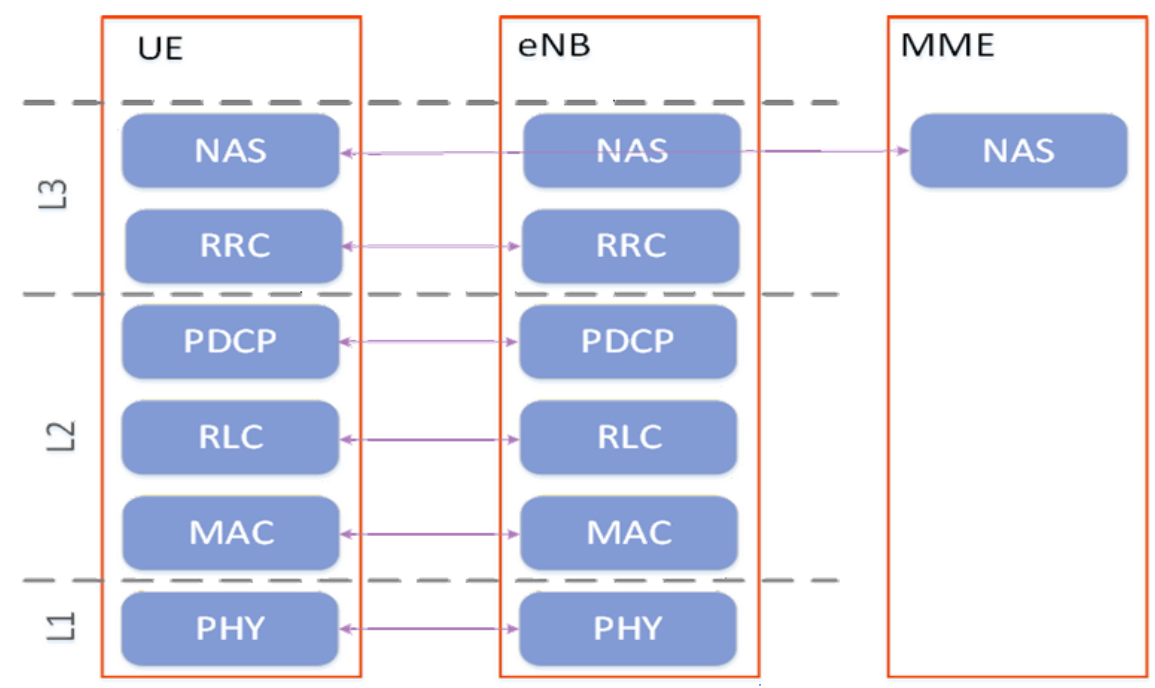

Figure 55: Control plane protocols

Figure 55 shows the control-plane protocol stack for the LTE and beyond cellular networks [35]. The main services and functions of the different sublayers (MAC, RLC and PDCP) of layers 2 are proving control channels for the transfer of control plane information. The 
radio resource control (RRC) and the non-access stratum (NAS) are responsible for three major control plane functions: (1) connectivity management, (2) mobility management and (3) radio resource management [35]. The RRC layer broadcasts the system information, establish, maintain and release a connection between the UE and the E-UTRAN. For the mobility management: UE measurement reporting (MR), handover decision, UE cell selection, establishment and release of radio bearer are also been done in RRC sublayer. Furthermore, for multi-cell cooperation: inter eNB communication, CSI feedback and exchange among the eNBs done in the control plane. The NAS sublayer provides connection/session management between the UE and the core network, authentication and location registration management. In this thesis, we studied coordination architecture and mobility management of cooperative communications of cellular networks to improve the performance of the system. 


\section{Appendix B Basic Transmission Scheme and Physical Resources}

In order to support flexible bandwidth and high data rate cellular networks adopted OFDMA from LTE networks. Downlink and uplink transmissions are organized into radio frames with $T_{f}=10 \mathrm{~ms}$ duration. Each frame consists of 10 subframes of $1 \mathrm{~ms}$ length, is the shortest interval for the transmission of resource blocks. A subframe consists of 2 slots, each of $0.5 \mathrm{~ms}$ length and each slot consists of 7 symbols of duration $T_{\text {symb }}=2048 \times T_{s}=$

$66.667 \mu s$. Where, $T_{s}=\frac{1}{N_{F F T} \times \Delta f}=\frac{1}{(2048 \times 15000)}$ seconds $=32.552 n s$ and $\mathrm{N}_{\mathrm{FFT}}=2048$ and $\Delta \mathrm{f}=15000 \mathrm{kHz}$. These seven symbols come with normal cyclic prefix (CP) length of $5.2083 \mu$ s for the first symbol and $4.6875 \mu$ s for the remaining six symbols. 


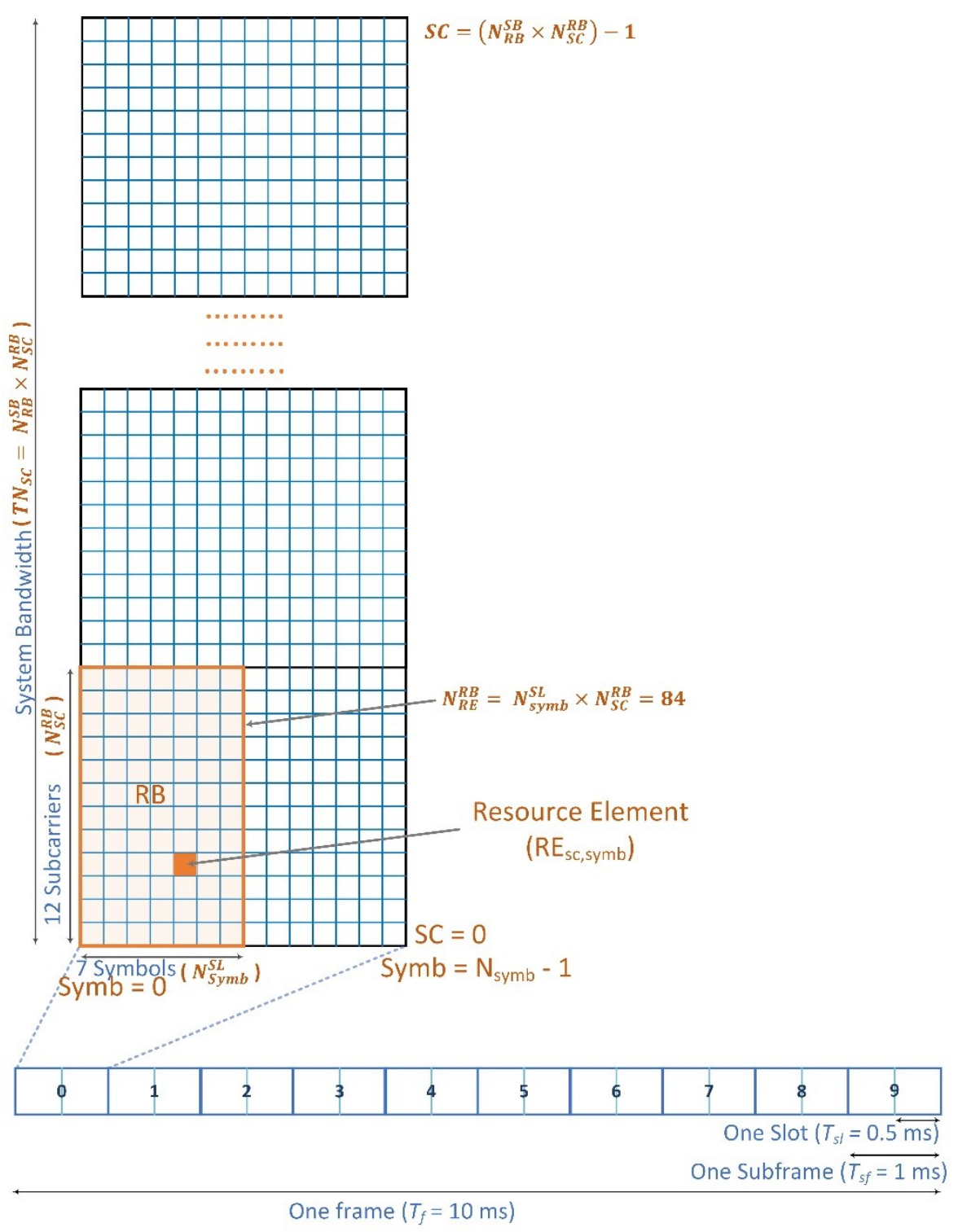

Figure 56: Transmission scheme and physical resource elements in wireless networks

As a global standard, LTE and beyond cellular networks adopt a wide variety of government specified spectral bands such as $1.4,3,5,10,15$ and $20 \mathrm{MHz}[81,151,152]$. Regardless of the total amount of bandwidth, LTE and beyond networks allocates timefrequency resources in terms of same resource unit called resource block (RB). An RB is composed of $N_{S y m b}^{S L}=7$ symbols and $N_{S C}^{R B}=12$ subcarriers. Each subcarrier $\Delta \mathrm{f}=15 \mathrm{kHz}$ as we stated before. Therefore, each RB consist of $180 \mathrm{kHz}$ in the frequency domain and 1 
slot which is $0.5 \mathrm{~ms}$ in the time domain. As a result, the total number of RBs varies according to the frequency bandwidth in use. The smallest resource unit is resource element (RE), which is defined as 1 subcarrier $\times 1$ symbol in the resource grid as shown in Figure 56. Therefore, the total number of RE in an RB is $12 \times 7=84$. Table 12 presents the summary of resource parameters in wireless networks.

Table 13: Resource parameters of LTE and beyond cellular networks

\begin{tabular}{|c|c|c|c|c|c|c|}
\hline & \multicolumn{6}{|c|}{ System Bandwidth } \\
\hline & $1.4 \mathrm{MHz}$ & $2 \mathrm{MHz}$ & $5 \mathrm{MHz}$ & $10 \mathrm{MHz}$ & $15 \mathrm{MHz}$ & $20 \mathrm{MHz}$ \\
\hline Subcarrier Size $\Delta f$ & & & & $15 \mathrm{kH}$ & & \\
\hline FFT size $\left(\mathbf{N}_{\text {FFT }}\right)$ & & & & 2048 & & \\
\hline $\begin{array}{c}\text { Number of Subcarriers } \\
\text { per RB }\left(N_{S C}^{R B}\right)\end{array}$ & & & & 12 & & \\
\hline $\begin{array}{l}\text { Number of Symbols per } \\
\qquad \operatorname{RB}\left(N_{S y m b}^{R B}\right)\end{array}$ & & & & 7 (normal & CP) & \\
\hline Number of $R B\left(N_{R B}^{S B}\right)$ & 6 & 15 & 25 & 50 & 75 & 100 \\
\hline $\begin{array}{l}\text { Number of Subcarriers } \\
\qquad\left(\boldsymbol{N}_{\boldsymbol{S C}}^{S B}\right)\end{array}$ & 72 & 180 & 300 & 600 & 900 & 1200 \\
\hline
\end{tabular}




\section{Appendix C Discrete Event System Specification}

The discrete event systems specifications (DEVS) is a formal modeling and simulation (M\&S) methodology for discrete event simulation models. It was introduced in 1976 to formalize the M\&S of dynamic systems based on system theory [29]. DEVS supports formal specifications for modeling both discrete and continuous systems. A DEVS model is organized hierarchically using modular descriptions, which facilitates construction, maintenance and reusability of the simulation while reducing the calculation time $[153$, 154]. In this appendix we provide a brief introduction about DEVS formalism.

A real world dynamic system modeled using DEVS can be described as a composition of atomic and coupled components. An atomic model represents a part of the system that describes the behavior of the part of the discrete event system as a sequence of deterministic transitions between states in response to the triggering of events. An atomic model changes its state if it receives an input via the input port or at the end of the time delay, whichever happens first. A coupled model is composed of several atomic or coupled sub-models; those are interconnected through the model's interface.

In the DEVS formalism, an atomic model is specified as follows [33, 29]:

$$
A M=\left\langle X, Y, S, \delta_{\text {int }}, \delta_{\text {ext }}, \lambda, t a\right\rangle,
$$

Equation 23

where,

$\mathrm{X}$ is a set of external input events, $\mathrm{Y}$ is a set of external output events and $\mathrm{S}$ is the set of states. $\delta_{\text {int }}: S \rightarrow S$ is the internal state transition function and $\delta_{\text {ext }}: Q \times X \rightarrow S$ is the external state transition function, where $Q=\{(s, e) \mid s \in S, 0 \leq e \leq t a(s)\}$ and $e$ is the elapsed time. The output function, $\lambda: S \rightarrow Y$ and the time advance function, $t a: S \rightarrow T$. 
According to the above specification, an atomic model is in a state $s \in S$ at any given time. The external transition function $\delta_{\text {ext }}$ invoked due to the reception of an input. That is, if the model experiences an external event, it processes the input and change to a new state according to the external transition function. If no external event happens, the model remains in the same state for a lifetime, defined by the $t a(s)$. When $t a(s)$ expires, an internal event occurs and an output may be processed that transmit through the output ports.

A coupled model is composed of atomic and coupled components as stated before. It is specified as follows $[33,29]$.

$$
C M=\langle X, Y, D, M, E I C, E O C, I C, \text { select }\rangle, \quad \text { Equation } 24
$$

where,

$\mathrm{X}$ is a set of input events and $\mathrm{Y}$ is a set of output events. $\mathrm{D}$ is a set of component IDs and $\mathrm{M}$ is a set of possible DEVS models (atomic or coupled). EIC is a set of external input couplings, EOC is a set of external output couplings and $I C$ is a set of internal couplings. The tiebreaker function is represented as select.

Figure 57 shows a simple DEVS model of one coupled model with two atomic models. The top model is the $e N B$ coupled model that consist of two atomic models, Buffer and Processor. The coupled model and the atomic models are interconnected through the 
input/output ports. Moreover, in Figure 57, Buffer and Processor also communicate with each other through $\mathrm{R}_{\text {out }}$ and $\mathrm{R}_{\text {in }}$ ports.

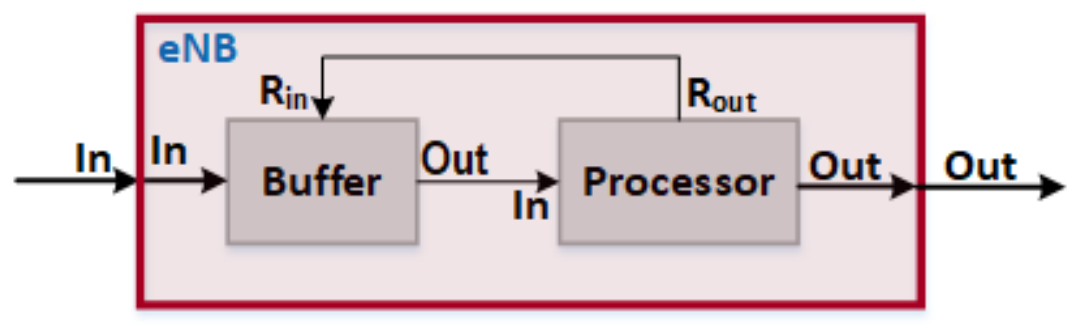

Figure 57: A simple DEVS coupled model with two atomic models

The $\mathrm{CD}++$ is an open source tool for discrete event modeling and simulation. It provides a development environment for implementing DEVS and Cell-DEVS models [33]. A DEVS atomic model can be developed and integrated onto a basic class hierarchy programmed in $\mathrm{C}++$. Coupled models can be defined using a built-in language according to the specifications.

As we said before, we use DEVS to develop simulation model for cellular networks employing proposed approaches. The DEVS formal specification provides a number of advantages for modelling and simulating of systems [29,33]. The hierarchical and modular nature of DEVS allows models to be extended and reused easily. Formal specification is useful to improve the security and reduce the development costs of a simulation. In this approach experimental framework (EF) is used as a testing module that improves model testing as well. Moreover, a well-defined separation of model, simulator and EF enables models and simulators to be validate and verified independently. 


\section{Bibliography}

[1] Ericsson, "Ericsson Mobility Report," 2018. [Online]. Available: https://www.ericsson.com/assets/local/mobility-report/documents/2018/emrinterim-feb-2018.pdf. [Accessed 26 March 2018].

[2] CISCO, "Cisco Visual Networking Index: Forecast and Methodology, 2016-2021," CISCO, 2017.

[3] M. H. Alsharif and R. Nordin, "Evolution towards fifth generation (5G) wireless networks: Current trends and challenges in the deployment of millimetre wave, massive MIMO, and small cells," Telecommunication Systems, pp. 1-21, 2016.

[4] M. Agiwal, R. Abhishek and S. Navrati, "Next generation 5G wireless networks: A comprehensive survey," IEEE Communications Surveys \& Tutorials, vol. 18, no. 3, pp. 1617-1655, 2016.

[5] Qualcomm, "1000x Data Challenge," 2014. [Online]. Available: https://www.qualcomm.com/invention/1000x/tools. [Accessed 15 august 2017].

[6] J. G. Andrews, Z. Xinchen, D. D. Gregory and K. G. Abhishek, "Are we approaching the fundamental limits of wireless network densification?," IEEE Communications Magazine, vol. 54, no. 10, pp. 184-190, 2016.

[7] A. Gotsis, S. Stelios and A. Angeliki, "UltraDense networks: The new wireless frontier for enabling 5G access," IEEE Vehicular Technology Magazine, vol. 11, no. 2, pp. 71-78, 2016. 
[8] M. Liu, T. Yinglei and S. Mei, "Performance Analysis of CoMP in Ultra-Dense Networks with Limited Backhaul Capacity," Wireless Personal Communications, vol. 91, no. 1, pp. 51-77, 2016.

[9] 3GPP, "3GPP TR 36.842 V12.0: Study on Small Cell enhancements for E-UTRA and E-UTRAN; Higher layer aspects," December 2013. [Online]. Available: http://www.3gpp.org/DynaReport/36-series.htm. [Accessed July 2016].

[10] D. Lopez-Perez, I. Guvenc, G. d. 1. Roche, M. Kountouris, T. Q. S. Quek and J. Zhang, "Enhanced intercell interference coordination challenges in heterogeneous networks," IEEE Wireless Communications, vol. 18, no. 3, pp. 22-30, 2011.

[11] S. Bassoy, F. Hasan, I. Muhammad A. and I. Ali, "Coordinated Multi-Point Clustering Schemes: A Survey," IEEE Communications Surveys \& Tutorials, vol. 19, no. 2, pp. 743-764, 2017.

[12] 3GPP, "3GPP TR 36.741 V14: Study on further enhancements to Coordinated Multi-Point (CoMP) operation for LTE," 23 March 2017. [Online]. Available: http://www.3gpp.org/DynaReport/36-series.htm. [Accessed 18 August 2017].

[13] S. Chen, Z. Tianyu, C. Hsiao-Hwa, L. Zhiping and M. Weixiao, "Performance Analysis of Downlink Coordinated Multipoint Joint Transmission in Ultra-Dense Networks," IEEE Network, 2017.

[14] M. Ding and H. Luo, Multi-point Cooperative Communication Systems: Theory and Applications, Shanghai Jiao Tong University Press, Shanghai and Springer-Verlag Berlin Heidelberg, 2013. 
[15] B. Özbek and D. L. Ruyet, "Feedback Strategies for Multicell Systems," in Feedback Strategies for Wireless Communication, New York, Springer, 2014, pp. 249-293.

[16] V. Garcia, Z. Yiqing and S. Jinglin, "Coordinated multipoint transmission in dense cellular networks with user-centric adaptive clustering," IEEE Transactions on Wireless Communications, vol. 13, no. 8, pp. 4297-4308, 2014.

[17] S. Sun, Q. Gao, Y. Peng, Y. Wang and L. Song, "Interference Management Through CoMP in 3GPP LTE-Advanced Networks," IEEE Wireless Communications, vol. 20, no. 1, pp. 59-66, 2013.

[18] P. Marsch and G. P. Fettweis, Coordinated Multi-Point in Mobile Communications: From Theory to Practice, Cambridge University Press, 2011.

[19] F. Qamar, B. D. Kaharudin, N. H. Mhd, A. B. N. Kamarul and M. A.-S. Ahmed, "A comprehensive review on coordinated multi-point operation for LTE-A," Computer Networks, vol. 123, pp. 19-37, 2017.

[20] Y. Wang and X. a. W. L. Jing, "A novel quantized time-domain compressed feedback scheme in coordinated multipoint LTE-advanced systems," Transactions on Emerging Telecommunications Technologies, vol. 28, no. 1, 2017.

[21] A. Papadogiannis, H. Bang, D. Gesbert and E. Hardouin, "Efficient Selective Feedback Design for Multicell Cooperative Networks," IEEE Transactions on Vehicular Technology, vol. 60, no. 1, pp. 196-205, 2011. 
[22] A. Papadogiannis, E. Hardouin and D. Gesbert, "Decentralising Multicell Cooperative Processing: A Novel Robust Framework," EURASIP Journal on Wireless Communications and Networking, vol. 2009, April 2009.

[23] I. F. Akyildiz, D. M. Gutierrez-Estevez and E. C. Reyes, "The evolution to 4G cellular systems: LTE-Advanced," Physical Communication, vol. 3, pp. 217-244, 2010.

[24] B. U. Kazi, G. A. Wainer, G. Boudreau and R. Casselman, "Coordinated MultiPoint (CoMP) Method And Systems Using a Coordination Station". US Patent P47112 US2, 2015.

[25] B. U. Kazi, M. Etemad, G. Wainer and G. Boudreau, "Using Elected Coordination Stations for CSI Feedback on CoMP Downlink Transmissions," in International Symposium on Performance Evaluation of Computer and Telecommunication Systems, Montreal, Canada , 2016.

[26] B. U. Kazi, M. Etemad, G. Wainer and G. Boudreau, "Signaling Overhead and Feedback Delay Reduction in Heterogeneous Multicell Cooperative Networks," in International Symposium on Performance Evaluation of Computer and Telecommunication Systems, Montreal, Canada, 2016.

[27] 3GPP, "3GPP TR 36.819 version 11.2.0: Coordinated multi-point operation for LTE physical layer aspects," 09 2013. [Online]. Available: http://www.3gpp.org/DynaReport/36-series.htm. [Accessed September 2016]. 
[28] J. Lee, Y. Kim, H. Lee, B. L. Ng, D. Mazzarese, J. Liu, W. Xiao and Y. Zhou, "Coordinated Multipoint Transmission and Reception in LTE-Advanced Systems," IEEE Communications Magazine, vol. 50, no. 11, pp. 44-50, 2012.

[29] B. Zeigler, H. Praehofer and T. Kim, Theory of modeling and simulation, San Diego, CA: Academic Press, 2000.

[30] B. U. Kazi and W. Gabriel, "Handover enhancement for LTE-Advanced and beyond heterogeneous cellular networks," in In International Symposium on Performance Evaluation of Computer and Telecommunication Systems (SPECTS), Seattle, WA, USA, 2017.

[31] B. U. Kazi and W. Gabriel, "Handover oscillation reduction in ultra-dense heterogeneous cellular networks using enhanced handover approach," in Proceedings of the Communications and Networking Symposium, Society for Computer Simulation International, Baltimore, Maryland, USA, 2018.

[32] 3GPP, "3GPP TR 36.839 V11.1: Evolved Universal Terrestrial Radio Access (EUTRA); Mobility enhancements in heterogeneous networks," 12 2012. [Online]. Available: http://www.3gpp.org/DynaReport/36-series.htm. [Accessed July 2016].

[33] G. A. Wainer, Discrete Event Modeling and Simulation A Practitioner's approach, Boca Raton, FL: CRC Press, Taylor \& Francis Group, 2009.

[34] G. A. Wainer, M. Tavanpour and E. Broutin, "Application of the DEVS and CellDEVS formalisms for modeling networking applications," in Proceedings of the Winter Simulation Conference (WSC), Washington, DC, 2013. 
[35] 3GPP, "3GPP TS 36.300 V14: Evolved Universal Terrestrial Radio Access (EUTRA) and Evolved Universal Terrestrial Radio Access Network (E-UTRAN); Overall description; Stage 2," September 2016. [Online]. Available: http://www.3gpp.org/DynaReport/36-series.htm. [Accessed October 2016].

[36] 3GPP, "3GPP TS 38.300 V15.0.0 NR; NR and NG-RAN Overall Description; stage-2; $\quad$ Release-15," $2018 . \quad$ [Online]. Available: http://www.3gpp.org/DynaReport/38-series.htm. [Accessed 3 2018].

[37] A. Gupta and R. K. Jha, "A survey of 5G network: Architecture and emerging technologies," IEEE access, vol. 3, pp. 1206-1232, 2015.

[38] K. R. Santhi, V. K. Srivastava, G. SenthilKumaran and B. Albert, "Goals of true broad band's wireless next wave (4G-5G)," in IEEE Vehicular Technology Conference, Orlando, FL, USA, 2003.

[39] C. Cox, An introduction to LTE: LTE, LTE-advanced, SAE and 4G mobile communications, John Wiley \& Sons, 2012.

[40] E. Hossain, M. Rasti, H. Tabassum and A. Abdelnasser, "Evolution toward 5G multi-tier cellular wireless networks: An interference management perspective," IEEE Wireless Communications, pp. 118-127, 2014.

[41] L. Song and S. Jia, Evolved cellular network planning and optimization for UMTS and LTE, CRC Press, 2010.

[42] Cisco, "Cisco Visual Networking Index: Global Mobile Data Traffic Forecast Update, 2015-2020," Cisco, 2016. 
[43] M. Peng, Y. Li, Z. Zhao and C. Wang, "System architecture and key technologies for 5G heterogeneous cloud radio access networks," IEEE Network, vol. 29, no. 2, pp. 6-14, 2015.

[44] C.-X. Wang, F. Haider, X. Gao, X.-H. You, Y. Yang, D. Yuan, H. Aggoune, H. Haas, S. Fletcher and E. Hepsaydir, "Cellular architecture and key technologies for 5G wireless communication networks," IEEE Communications Magazine, vol. 52, no. 2, pp. 122 - 130, 2014.

[45] P. K. Agyapong, I. Mikio, S. Dirk, K. Wolfgang and B. Anass, "Design considerations for a 5G network architecture," IEEE Communications Magazine, vol. 52, no. 11, pp. 65-75, 2014.

[46] GSMA Intelligence, "Understanding 5G: Perspectives on future technological advancements in mobile," December 2014.

[47] B. U. Kazi and A. W. Gabriel, "Next generation wireless cellular networks: ultradense multi-tier and multi-cell cooperation perspective," Wireless Networks, pp. 124, 2018.

[48] Ericsson , "5G systems," January 2017. [Online]. Available: https://www.ericsson.com/assets/local/publications/white-papers/wp-5gsystems.pdf. [Accessed 12 August 2017].

[49] M. Liu, T. Yinglei and S. Mei, "Performance analysis of coordinated multipoint joint transmission in ultra-dense networks with limited backhaul capacity," IET Electronics Letters, vol. 51, no. 25, pp. 2111-2113, 2015. 
[50] D. López-Pérez, D. Ming, C. Holger and J. Amir H., "Towards 1 Gbps/UE in cellular systems: Understanding ultra-dense small cell deployments," IEEE Communications Surveys \& Tutorials, vol. 17, no. 4, pp. 2078-2101, 2015.

[51] M. Ding, L. P. David and M. Guoqiang, "A new capacity scaling law in ultra-dense networks," in arXiv preprint arXiv:1704.00399, 2017.

[52] M. Ding, L.-P. David, M. Guoqiang, W. Peng and L. Zihuai, "Will the area spectral efficiency monotonically grow as small cells go dense," in Global Communications Conference (GLOBECOM), 2015 IEEE, San Diego, USA, 2015.

[53] J. Ghosh, D. N. K. Jayakody and T. Tsiftsis A., "Coverage Probability Analysis by Fractional Frequency Reuse Scheme," in 1st International Telecommunications conference ITELCON 2017 (Springer Lecture Notes in Electrical Engineering), Istanbul, Turkey, 2017.

[54] P. Kela, T. Jussi and M. Costa, "Borderless mobility in 5G outdoor ultra-dense networks," IEEE Access, vol. 3, pp. 1462-1476, 2015.

[55] J. Zhang, F. Jian, L. Chang, H. Xuefen, Z. Xing and W. Wang, "Mobility enhancement and performance evaluation for 5G Ultra dense Networks," in Wireless Communications and Networking Conference (WCNC), 2015 IEEE, New Orleans, LA, USA, 2015.

[56] H. Wang, C. Shanzhi, M. Ai and X. U. Hui, "Localized Mobility Management for 5G Ultra Dense Network," IEEE Transactions on Vehicular Technology, no. 99, 2017. 
[57] B. Romanous, N. Bitar, A. Imran and H. Refai, "Network densification: Challenges and opportunities in enabling 5G," in IEEE 20th International Workshop on in Computer Aided Modelling and Design of Communication Links and Networks (CAMAD), Guildford, UK, 2015.

[58] M. Gao, J. Li, D. N. Jayakody, H. Chen, Y. Li and J. Shi, "A Super Base Station Network Architecture for Ultra-Dense Networks," IEEE Communications Magazine, 2017.

[59] X. Ge, S. Tu, G. Mao, C.-X. Wang and T. Han, "5G ultra-dense cellular networks," Ge, Xiaohu, Song Tu, Guoqiang Mao, Cheng-Xiang Wang, and IEEE Wireless Communications, vol. 23, no. 1, pp. 72-79, 2016.

[60] Z. Gao, D. Linglong, M. De, W. Zhaocheng, A. I. Muhammad and Z. S. Muhammad, "MmWave massive-MIMO-based wireless backhaul for the 5G ultradense network," IEEE Wireless Communications, vol. 22, no. 5, pp. 13-21, 2015.

[61] M. Jaber, A. I. Muhammad, T. Rahim and T. Anvar, "5G backhaul challenges and emerging research directions: A survey," IEEE Access, vol. 4, pp. 1743-1766, 2016.

[62] O. Galinina, A. Pyattaev, AndreevS, M. Dohler and y. Koucheryavy, "5G multiRAT LTE-WiFi ultra-dense small cells: Performance dynamics, architecture, and trends," IEEE Journal on Selected Areas in Communications, vol. 33, no. 6, pp. 1224-1240, 2015.

[63] M. Kamel, H. Walaa and Y. Amr, "Ultra-dense networks: A survey," IEEE Communications Surveys \& Tutorials, vol. 18, no. 4, pp. 2522-2545, 2016. 
[64] A. Damnjanovic, M. Juan, W. Yongbin, J. Tingfang, L. Tao, V. Madhavan, Y. Taesang, S. Osok and M. Durga, "A survey on 3GPP heterogeneous networks," IEEE Wireless Communications, vol. 18, no. 3, pp. 10-21, 2011.

[65] Nokia, "Ultra Dense Network (UDN)," 2016. [Online]. Available: https://resources.ext.nokia.com/asset/200295. [Accessed 4 August 2017].

[66] S. Chen, F. Qin, B. Hu, X. Li and J. Liu, "Ultra-Dense Network Architecture and Technologies for 5G," in 5G Mobile Communications, Springer International Publishing, 2017, pp. 403-429.

[67] N. Wang, H. Ekram and B. Vijay K., "Backhauling 5G small cells: A radio resource management perspective," IEEE Wireless Communications, vol. 22, no. 5, pp. 4149, 2015.

[68] X. Zhang and X. Zhou, LTE-Advanced Air Interface Technology, CRC Press, 2012.

[69] Y. Zhou, L. Liu, H. Du, L. Tian, X. Wang and J. Shi, "An overview on intercell interference management in mobile cellular networks: From $2 \mathrm{G}$ to $5 \mathrm{G}$," in Communication Systems (ICCS), 2014 IEEE International Conference on, Macau, China, 2014.

[70] 3GPP, "3GPP TS 36.331 Release 14: Radio Resource Control (RRC) protocol specification," 26 September 2017. [Online]. Available: http://www.3gpp.org/DynaReport/36-series.htm.. [Accessed July 2017].

[71] A. Papadogiannis, E. Hardouin, and D. Gesbert, "DecentralisingMulticell Cooperative Processing: A Novel Robust Framework," EURASIP Journal onWireless Communications and Networking, April 2009. 
[72] I. F. Akyildiz, G.-E. David M., B. Ravikumar and E. Chavarria-Reyes, "LTEAdvanced and the evolution to Beyond 4G (B4G) systems," Physical Communication, vol. 10, pp. 31-60, 2014.

[73] S. Sun, Q. Gao, Y. Peng, Y. Wang and L. Song, "Interference Management Through CoMP in 3GPP LTE-Advanced Networks," IEEE Wireless Communications, vol. 20, no. 1, 2013.

[74] Y.-N. R. Li, J. Li, W. Li, Y. Xue and H. Wu., "CoMP and interference coordination in heterogeneous network for LTE-advanced," in 2012 IEEE Globecom Workshops, pp. 1107-1111. IEEE, 2012., California, USA, December, 2012.

[75] H. Zhang, J. Chunxiao, C. Julian and C. L. Victor, "Cooperative interference mitigation and handover management for heterogeneous cloud small cell networks," IEEE Wireless Communications, vol. 22, no. 3, pp. 92-99, 2015.

[76] S. Chen, Z. Tianyu, C. Hsiao-Hwa, Z. Lu and M. Weixiao, "Performance Analysis of Downlink Coordinated Multipoint Joint Transmission in Ultra-Dense Networks," IEEE Network, vol. pp, no. 99, pp. 12 - 20, 2017.

[77] A. G. Gotsis, S. Stelios and A. Angeliki, "Spatial coordination strategies in future ultra-dense wireless networks," in 11th International Symposium on Wireless Communications Systems (ISWCS), Barcelona, Spain, 2014.

[78] J. Liu and X. Weimin, "Advanced carrier aggregation techniques for multi-carrier ultra-dense networks," IEEE Communications Magazine, vol. 54, no. 7, pp. 61-67, 2016. 
[79] J. Lee, Y. Kim, H. Lee, B. L. Ng, D. Mazzarese, J. Liu, W. Xiao and Y. Zhou, "Coordinated Multipoint Transmission and Reception in LTE-Advanced Systems," IEEE Communications Magazine, 2012.

[80] M. Ding and H. Luo, Multi-point Cooperative Communication Systems: Theory and Applications, Shanghai Jiao Tong University Press, Shanghai and Springer-Verlag Berlin Heidelberg, 2013.

[81] 3GPP, "3GPP TS 36.211 version 15.0: Evolved Universal Terrestrial Radio Access (E-UTRA); Physical channels and modulation," 12 2017. [Online]. Available: http://www.3gpp.org/DynaReport/36-series.htm. [Accessed February 2018].

[82] 3GPP, "TS 36.300 V15: Evolved Universal Terrestrial Radio Access (E-UTRA) and Evolved Universal Terrestrial Radio Access Network (E-UTRAN); Overall description; Stage 2," December 2017. [Online]. Available: http://www.3gpp.org/DynaReport/36-series.htm. [Accessed January 2018].

[83] Y.-H. Nam, A. Yosuke, K. Younsun, L. Moon-il, B. Kapil and E. Anthony, "Evolution of reference signals for LTE-advanced systems," IEEE Communications Magazine, vol. 50, no. 2, pp. 132-138, 2012.

[84] P. Marsch and G. P. Fettweis, Coordinated Multi-Point in Mobile Communications: from theory to practice, Cambridge University Press, 2011.

[85] A. F. Molisch, Wireless communications, John Wiley \& Sons, 2012.

[86] 3GPP, "3GPP TS 36.213 V15.1.0: Evolved Universal Terrestrial Radio Access (EUTRA), Physical layer procedures (Release 15)," April 2018. [Online]. Available: http://www.3gpp.org/DynaReport/36-series.htm. [Accessed 2018]. 
[87] A. Chiumento, C. Desset, P. Sofie, V. d. P. Liesbet and R. Lauwereins, "Impact of CSI feedback strategies on LTE downlink and reinforcement learning solutions for optimal allocation," IEEE Transactions on Vehicular Technology , vol. 66, no. 1, pp. 550-562, 2017.

[88] S. Bassoy, J. Mona, A. I. Muhammad and X. Pei, "Load aware self-organising usercentric dynamic CoMP clustering for 5G networks," IEEE Access, vol. 4, pp. 2895 $2906,2016$.

[89] C.-Y. Wei and W. Liao, "Multi-Cell Cooperative Scheduling for Network Utility Maximization With User Equipment Side Interference Cancellation," IEEE Transactions on Wireless Communications, vol. 17, no. 1, pp. 619-635, 2018.

[90] S. Jain, S.-J. Kim and G. B. Giannakis, "Backhaul-constrained multicell cooperation leveraging sparsity and spectral clustering," IEEE Transactions on Wireless Communications, vol. 15, no. 2, pp. 899-912, 2016.

[91] T. Biermann, S. Luca, C. Changsoon, K. Holger and K. Wolfgang, "CoMP clustering and backhaul limitations in cooperative cellular mobile access networks," Pervasive and Mobile Computing, vol. 8, no. no, pp. 662-681, 2012.

[92] T. Biermann, L. Scalia, C. Choi, K. Wolfgang and H. Karl, "How backhaul networks influence the feasibility of coordinated multipoint in cellular networks," IEEE Communications Magazine 51, no. 8 (2013):, vol. 51, no. 8, pp. 168-176, 2013.

[93] U. Siddique, T. Hina, H. Ekram and I. K. Dong, "Wireless backhauling of 5G small cells: challenges and solution approaches," IEEE Wireless Communications, vol. 22, no. 22, pp. 22-31, 2015. 
[94] Small Cell Forum, "Backhaul technologies for small cells: Use cases, requirements and solution," Small Cell Forum, Tech. Rep. 049.01.01, OK, 2013.

[95] 3GPP, "TS 36.420: Evolved Universal Terrestrial Radio Access Network (EUTRAN); X2 general aspects and principles," March 2017. [Online]. Available: http://www.3gpp.org/DynaReport/36-series.htm. [Accessed 14 July 2017].

[96] D. H. Nguyen and L.-N. Tho, Wireless coordinated multicell systems: Architectures and precoding designs, Montréal, QC, Canada: Springer, 2014.

[97] 3GPP, "TS 38.420 V0.6.0: NG-RAN; Xn general aspects and principles," 032018. [Online]. Available: http://www.3gpp.org/DynaReport/38-series.htm. [Accessed 03 2018].

[98] Y. Yang, W. S. Ki, P. Jihong, K. Seong-Lyun and K. Kwang Soon, "Cooperative Transmissions in Ultra-Dense Networks under a Bounded Dual-Slope Path Loss Model," arXiv preprint arXiv, 2017.

[99] T. H. Naylor and J. M. Finger, "Verification of computer simulation models," Management Science, vol. 14, no. 2, pp. B92-B106, 1967.

[100] B. Jerry, Discrete-event system simulation, Pearson Education, 1984.

[101] O. Balci, "Credibility assessment of simulation results," in In Proceedings of the 18th conference on Winter simulation, 1986.

[102] O. Balci, "Verification, validation, and certification of modeling and simulation applications," in In Proceedings of the 35th conference on Winter simulation, 2003.

[103] R. E. Shannon, "Introduction to the art and science of simulation," in Proceedings of the 30th conference on Winter simulation, 1998. 
[104] R. E. Shannon, Systems simulation; the art and science, 1975.

[105] R. G. Sargent, "Verification and validation of simulation models," Journal of simulation, vol. 7, no. 1, pp. 12-24, 2013.

[106] F. Neelamkavil, Computer simulation and modelling, John Wiley \& Sons, Inc, 1987.

[107] W. L. a. C. J. R. Oberkampf, Verification and validation in scientific computing, Cambridge University Press, 2010..

[108] Y. Barlas, "Formal aspects of model validity and validation in system dynamics," System dynamics review, vol. 12, no. 3, pp. 183-210, 1996.

[109] S. Schlesinger, "Terminology for model credibility," Simulation, vol. 32, no. 3, pp. 103-104, 1979.

[110] DoD, "DoD Instruction 5000.61: DoD Modeling and Simulation (M\&S) Verification.Validation, and Accreditation (VV\&A)," 1996.

[111] G. Cili, H. Yanikomeroglu and F. R. Yu, "Cell Switch Off Technique Combined with Coordinated Multi-Point (CoMP) Transmission for Energy Efficiency in Beyond-LTE Cellular Networks," in Communications (ICC), 2012 IEEE International Conference on, Ottawa, ON, 2012.

[112] A. Davydov, M. Gregory, B. Ilya and A. Papathanassiou, "Evaluation of joint transmission CoMP in C-RAN based LTE-A HetNets with large coordination areas," in IEEE Globecom Workshops (GC Wkshps), 2013 IEEE, pp. 801-806. IEEE, 2013., Atlanta, GA, USA, 2013. 
[113] H. Ishii, K. Yoshihisa and T. Hideaki, "A novel architecture for LTE-B: C-plane/Uplane split and phantom cell concept," in IEEE Globecom Workshops, California, USA, 2012.

[114] 3GPP, "3GPP TR 36.942 V14.0: Evolved Universal Terrestrial Radio Access (EUTRA); Radio Frequency (RF) system scenarios," 01 2017. [Online]. Available: http://www.3gpp.org/DynaReport/36-series.htm. [Accessed April 2017].

[115] 3GPP, "3GPP TS 36.211 version 14.0: Evolved Universal Terrestrial Radio Access (E-UTRA); Physical channels and modulation," 09 2016. [Online]. Available: http://www.3gpp.org/DynaReport/36-series.htm. [Accessed February 2016].

[116] 3GPP, "3GPP TS36.212, V15.1.0 :Evolved Universal Terrestrial Radio Access (EUTRA); Multiplexing and channel coding," 4 2018. [Online]. Available: http://www.3gpp.org/DynaReport/36-series.htm. [Accessed 5 2018].

[117] B. Mondal, E. Visotsky, T. Thomas, X. Wang and A. Ghosh, "Performance of downlink comp in LTE under practical constraints," in IEEE 23rd International Symposium on Personal Indoor and Mobile Radio Communications (PIMRC), Sydney, NSW , 2012.

[118] C. Yang, H. Shengqian, H. Xueying and M. Andreas, "How do we design CoMP to achieve its promised potential," IEEE Wireless Communications, vol. 20, no. 1, pp. 67-74, 2013.

[119] S. Brueck, L. Zhao, J. Giese and A. Amin, "Centralized Scheduling for Joint Transmission Coordinated Multi-Point in LTE-Advanced," in International ITG Workshop on Smart Antennas (WSA), Bremen, 2010. 
[120] SalesForce, "2014 Mobile Behavior Report," SalesForce, 2014.

[121] B. Mielczarek and W. Krzymien, "Influence of CSI Feedback Delay on Capacity of Linear Multi-User MIMO Systems," in IEE Wireless Communications and Networking Conference, Kowloon, 2007.

[122] L. Shi, H. Zhirui, Z. Tiankui and Z. Zeng, "Performance analysis of delayed limited feedback based on per-cell codebook in CoMP systems," in In Wireless Communications and Networking Conference (WCNC), 2015 IEEE, New Orleans, LA, USA, 2015.

[123] Rakon, "Small Cells Solutions," 2015. [Online]. Available: http://www.rakon.com/products/technical-resources/tech-docs. [Accessed 15 August 2017].

[124] D. Lopez-Perez, İ. Güvenc and a. X. Chu., "Mobility management challenges in 3GPP heterogeneous networks," Communications Magazine, vol. 50, no. 12, pp. 70$78,2012$.

[125] 3GPP, "3GPP TS 36.300 V14: Evolved Universal Terrestrial Radio Access Network (E-UTRAN)," September 2016. [Online]. Available: http://www.3gpp.org/DynaReport/36-series.htm. [Accessed October 2016].

[126] B. U. Kazi, G. Wainer and V. Guimaraes da Silva, "Modeling and Simulation of User Mobility and Handover in LTE and Beyond Mobile Networks Using DEVS Formalism," in Communications and Networking Symposium (CNS2017), Virginia Beach, VA USA, 2017. 
[127] 3GPP, "3GPP TS 36.133 V14: Evolved Universal Terrestrial Radio Access (EUTRA); Base Station (BS) and repeater ElectroMagnetic Compatibility (EMC)," 2016-06. [Online]. Available: http://www.3gpp.org/DynaReport/36-series.htm. [Accessed August 2016].

[128] K. Vasudeva, M. Simsek, D. López-Pérez and I. Guvenc, "Analysis of Handover Failures in Heterogeneous Networks with Fading," Vasudeva, Karthik, Meryem Simsek, David López-Pérez, and Ismail Guvenc. "Analysi arXiv preprint arXiv:1507.01586, 2015.

[129] Q. Kuang, B. Jakob, B. Zarah, D. Heinz and S. Joachim, "A measurement-based study of handover improvement through range expansion and interference coordination," Wireless Communications and Mobile Computing, vol. 15, no. 14, pp. 1784-1798, 2015.

[130] 3GPP, "3GPP TR 36.932 V13: Scenarios and requirements for small cell enhancements for E-UTRA and E-UTRAN," 2016a. [Online]. Available: http://www.3gpp.org/DynaReport/36-series.htm. [Accessed June 2017].

[131] 3GPP, "TSG-RAN WG2 Meeting \#81bis, R2-131087: Mobility performance for small cell deployment scenario 2," 2013c. [Online]. Available: http://www.3gpp.org/ftp/tsg_ran/wg2_rl2/TSGR2_81bis/Docs/. [Accessed March 2017]. 
[132] B. U. Kazi, M. Etemad, G. Wainer and G. Boudreau, "Signaling Overhead and Feedback Delay Reduction in Heterogeneous Multicell Cooperative Networks," in International Symposium on Performance Evaluation of Computer and Telecommunication Systems, Montreal, Canada, 2016a.

[133] 3GPP, "3GPP TR 36.942 V13.0: Evolved Universal Terrestrial Radio Access (EUTRA); Radio Frequency (RF) system scenarios," 2016b. [Online]. Available: http://www.3gpp.org/DynaReport/36-series.htm. [Accessed April 2016].

[134] 3GPP, "3GPP TS 36.814 V9.2.0: Evolved Universal Terrestrial Radio Access (EUTRA); Further advancements for E-UTRA physical layer aspects," March 2017. [Online]. Available: http://www.3gpp.org/DynaReport/36-series.htm. [Accessed July 2017].

[135] B. P. Zeigler and N. James J., "Towards a framework for more robust validation and verification of simulation models for systems of systems," The Journal of Defense Modeling and Simulation, vol. 13, no. 1, pp. 3-16, 2016.

[136] S. Robinson, "Simulation model verification and validation: increasing the users' confidence," Proceedings of the 29th conference on Winter simulation, IEEE Computer Society, pp. 53-59, 1997.

[137] Y. Barlas, "Formal aspects of model validity and validation in system dynamics," System dynamics review, vol. 12, no. 3, pp. 183-210, 1996. 
[138] R. J. Gore, L. Christopher J. and K. Hamdi, "Applying statistical debugging for enhanced trace validation of agent-based models," Simulation: Transactions of the Society for Modeling and Simulation International, vol. 93, no. 4, pp. 273-284, 2017.

[139] T. I. Ören and P. Z. Bernard, "Concepts for advanced simulation methodologies," Simulation: Transactions of The Society for Modeling and Simulation International, vol. 32, no. 3, pp. 69-82., 1979.

[140] T. Daum and S. Robert G., "Experimental frames in a modern modeling and simulation system.," IIE Transactions, vol. 33, no. 3, pp. 181-192, 2001.

[141] L. E. Schwer, "Guide for verification and validation in computational solid mechanics," in 20th International Conference on Structural Mechanics in Reactor Technology (SMiRT 20), Espoo, Finland, August 9-14, 2009.

[142] P. Knupp and S. Kambiz, Verification of computer codes in computational science and engineering, CRC Press, 2002.

[143] C. J. Roy and O. William L., "A comprehensive framework for verification, validation, and uncertainty quantification in scientific computing," Computer methods in applied mechanics and engineering, vol. 200, no. 25-28, pp. 2131-2144, 2011.

[144] A. Inostrosa-Psijas, W. Gabriel, G.-C. Veronica and M. Marin, "DEVs modeling of large scale web search engines," in Winter Simulation Conference (WSC), Savanah, GA, USA, 2014. 
[145] R. G. Sargent, "Verification and validation of simulation models," Procedings of Winter Simulation Conference, IEEE, 2007., pp. 124-137, 2007.

[146] J. Lim and H. Daehyoung, "Mobility and handover management for heterogeneous networks in LTE-advanced," Wireless personal communications, vol. 72, no. 4, pp. 2901-2912, 2013.

[147] 3GPP, "3GPP TR:36.814 Evolved Universal Terrestrial Radio Access (E-UTRA); Further advancements for E-UTRA physical layer aspects," March 2017. [Online]. Available: http://www.3gpp.org/DynaReport/36-series.htm. [Accessed September 2017].

[148] H.-D. Bae, R. ByungHan and P. Nam-Hoon, "Analysis of handover failures in LTE femtocell systems," in 2011 Australasian Telecommunication Networks and Applications Conference (ATNAC), IEEE, Melbourne, VIC, Australia, 2011.

[149] 3GPP, "R2-131087, Mobility performance for small cell deployment," April 2013. [Online].

Available: http://www.3gpp.org/ftp/tsg_ran/wg2_rl2/TSGR2_81bis/Docs/. [Accessed july 2015].

[150] T. S. Rappaport, Wireless communications: principles and practice, New Jersey: Prentice Hall PTR, 1996.

[151] G. Ku and M. W. John, "Resource allocation and link adaptation in LTE and LTE advanced: A tutorial," IEEE Communications Surveys \& Tutorials, vol. 17, no. 3, pp. 1605-1633, 2015. 
[152] 3GPP, "Evolved TS 36.101, V15.1.0: Universal Terrestrial Radio Access (EUTRA); User Equipment (UE) radio transmission and reception (Release 15)," Jan 2018. [Online]. Available: http://www.3gpp.org/DynaReport/36-series.htm.

[153] A. Muzy, E. Innocenti, A. Aiello, J.-F. Santucci, T. Marcelli and a. P. A. Santoni, "Discrete-event modelling of fire spreading," International Journal of Systems Science, p. 193-206, 2008.

[154] B. U. Kazi and G. Wainer, "Integrated cellular framework for modeling ecosystems: Theory and applications," SIMULATION: The tr, 2017. 